\title{
MEASUREMENT OF THE TARGET SINGLE-SPIN ASYMMETRY IN QUASI-ELASTIC REGION FROM THE REACTION ${ }^{3} \mathrm{He}^{\uparrow}\left(e, e^{\prime}\right)$
}

BY YAWEI ZHANG

\author{
A dissertation submitted to the \\ Graduate School-New Brunswick \\ Rutgers, The State University of New Jersey \\ in partial fulfillment of the requirements \\ for the degree of \\ Doctor of Philosophy \\ Graduate Program in Physics and Astronomy \\ Written under the direction of \\ Prof. Ronald Gilman \\ and approved by
}

New Brunswick, New Jersey

October, 2013 


\section{ABSTRACT OF THE DISSERTATION}

\section{Measurement of the Target Single-Spin Asymmetry in Quasi-Elastic Region from the Reaction ${ }^{3} \mathbf{H e}^{\uparrow}\left(e, e^{\prime}\right)$}

\section{by Yawei Zhang \\ Dissertation Director: Prof. Ronald Gilman}

A measurement of the inclusive target single-spin asymmetry, $A_{y}^{3} \mathrm{He}$, has been performed using the quasi-elastic ${ }^{3} \mathrm{He}^{\uparrow}\left(e, e^{\prime}\right)$ reaction with a vertically polarized ${ }^{3} \mathrm{He}$ target at $Q^{2}$ values of $0.13,0.46$ and $0.97 \mathrm{GeV}^{2}$. This asymmetry vanishes under the one photon exchange assumption. But the interference between two-photon exchange and one-photon exchange gives rise to an imaginary amplitude, so that a non-zero $A_{y}$ is allowed. The experiment, conducted in Hall A of Jefferson Laboratory in 2009, used two independent spectrometers to simultaneously measure the target single-spin asymmetry, $A_{y}^{3} \mathrm{He}$.

Using the effective polarization approximation, the neutron single-spin asymmetries, $A_{y}^{n}$, were extracted from the measured ${ }^{3} \mathrm{He}$ asymmetries. The measurement is the first to firmly establish a non-vanishing $A_{y}$. Non-zero asymmetries were observed at all $Q^{2}$ points, and the final overall precision is an order of magnitude improved over the existing proton data. The data provide new constraints 
on Generalized Parton Distribution (GPD) models [1] and new information on the dynamics of the two-photon exchange process. 


\section{Acknowledgements}

There are several people have helped me in my doctoral research, which has made my journey to the finish line possible. As I am about to finish this thesis, I would like to express my sincere thanks to all of them:

First of all, I would like to express my sincere gratitude to Prof. Ronald Gilman for his excellent advising and the research opportunity he gave me to participate in the experiments at Jefferson Lab. He is a wonderful mentor, who gave me a completely autonomous freedom, and let me do the research I wanted. However, from time to time to hear discussions helped to point me in the right direction, I benefit from these discussions over the years. I would like to thank him for sacrificing a lot of time to read my thesis and point out the mistakes that I made. Without his patient instruction, insightful criticism and expert guidance, the completion of this thesis would not have been possible.

Second, I would like to thank Dr. Jian-ping Chen and Dr. Douglas Higinbotham for their guidance and advice on my work at Jefferson Lab, they supervised me through every step of my thesis work and gave me valuable advice. I have always enjoyed fruitful discussions with them and benefited during my data analysis process. I thank other spokespersons of this experiment, Dr. Xiaodong Jiang and Prof. Todd Averett for their leadership in this experiment.

Third, I thank the postdocs of this experiment, Alexandre Camsonne, Lamiaa El Fassi, Aidan Kelleher, Yi Qiang, Vince Sulkosky, Brad Sawatzky and Bo Zhao, as well as the Run Coordinators, for their excellent service and kind help on my research, from the apparatus setup to the data analysis. Especially for Vince who has answered so many of my questions, Lamiaa who worked with me on the 
polarized ${ }^{3}$ He target, and Bo who guided me into the first step of data analysis. I would like to thank my fellow graduate students Dr. Ge Jin, Dr. Elena Long, and Dr. Miha Mihovilovic. Our experiments would not have been possible without the countless hours spent in the counting house, working on equipment, and data analysis by them.

Moreover, I would like to thank the students Dr. Kalyan Allada, Dr. Chiranjib Dutta, Dr. Jin Huang, Dr. Joseph Katich, Dr. Xin Qian, Dr. Youcai Wang, Dr. Yi Zhang, and students David Flay, Dr. Diana Parno, Matthew Posik, not only for their help in the experiment, but also for answering my questions and giving me suggestions during the data analysis. I thank Dr. Alexandre Deur and Dr. Patricia Solvignon for discussions on radiative corrections, Prof. Andrei Afanasev, Prof. Arnoldas Deltuva and Prof. Simon Sirca for discussions on the extraction of the neutron asymmetry.

Finally, I would like to dedicate this thesis to my beloved parents, for all their support and patience through many years of school away from home. 


\section{Dedication}

To my parents 


\section{Table of Contents}

Abstract ................................. ii

Acknowledgements .......................... iv

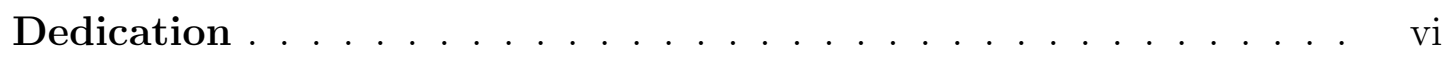

List of Tables . . . . . . . . . . . . . . . . . . . . . . . . xii

List of Figures . . . . . . . . . . . . . . . . . . . xiv

1. Introduction . . . . . . . . . . . . . . . . . 1

1.1. Fundamental Constituents of Matter . . . . . . . . . . . . . . . 1

1.2. Fundamental Interactions . . . . . . . . . . . . . . 2

1.3. Why Electron Scattering . . . . . . . . . . . . . . . . 4

1.4. Outline of the Dissertation . . . . . . . . . . . . . . 5

2. General Formalism . . . . . . . . . . . . . . . . . . . . 7

2.1. Cross-Section . . . . . . . . . . . . . . . . 7

2.2. Feynman Diagram ..................... . . 8

2.3. Kinematic Variables . . . . . . . . . . . . . . . . . . . . . . . 10

2.4. The Rutherford and Mott Cross-Sections . . . . . . . . . . . . . . 12

2.5. Nuclear Form Factors . . . . . . . . . . . . . . . . . . . . . . . . . 13

2.6. Types of Inclusive Electron Scattering _ . . . . . . . . . . . . . . 15

2.6.1. Elastic Scattering off Nucleons . . . . . . . . . . . . . . . 15

2.6.2. Quasi-Elastic Scattering . . . . . . . . . . . . 18

2.6.3. Resonances . . . . . . . . . . . . . . . . . 19 
2.6.4. Deep Inelastic Scattering . . . . . . . . . . . . . . . . . . 19

2.7. Tensor Formulation . . . . . . . . . . . . . . . . . . . . . 20

3. Two Photon Physics . . . . . . . . . . . . . . . . 24

3.1. The Born Approximation. . . . . . . . . . . . . . . . . . 24

3.2. The Importance of Two-Photon Physics $\ldots \ldots \ldots$

3.3. Experimental Observable for Two Photon Exchange . . . . . . . . 30

3.4. Define Up and Down . . . . . . . . . . . . . . . . . . . 30

3.5. Existing Data . . . . . . . . . . . . . . . . . . . . . 31

3.6. Jefferson Lab E05-015 . . . . . . . . . . . . . . . . . . . . 31

4. Experimental Setup . . . . . . . . . . . . . . . . . . . 34

4.1. Jefferson Lab . . . . . . . . . . . . . . . . . . . . . . . . . 34

4.2. The Continuous Electron Beam Accelerator Facility . . . . . . . . 36

$4.2 .1 . \quad$ Injector $\ldots \ldots \ldots \ldots \ldots \ldots \ldots \ldots$

4.2.2. Linear Accelerator $\ldots \ldots \ldots$. . . . . . . . . 37

$4.2 .3 . \quad$ Recirculating Arcs $\ldots \ldots \ldots \ldots$

$4.2 .4 . \quad$ Beam Switchyard . . . . . . . . . . . . . . . . 38

$4.3 . \quad$ Experimental Hall $\mathrm{A} \ldots \ldots \ldots$

4.4. Beam Line . . . . . . . . . . . . . . . . . . . . . . . . 40

4.4.1. Beam Energy Measurement . . . . . . . . . . . . . . 40

4.4.2. Beam Current Measurement . . . . . . . . . . . . . . . 42

4.4.3. Beam Position Measurement . . . . . . . . . . . . . . . . 43

4.4.4. Beam Raster . . . . . . . . . . . . . . . . 45

4.5. High Resolution Spectrometers _. . . . . . . . . . . . . . 45

$4.5 .1 . \quad$ Detector Package . . . . . . . . . . . . . . . . . 46

4.5.2. Vertical Drift Chambers _. . . . . . . . . . . . 49

4.5.3. Scintillator Planes and Trigger Electronics . . . . . . . . 49 
4.5.4. Gas Cherenkov Detector . . . . . . . . . . . . . . 52

4.5.5. Lead Glass Counters . . . . . . . . . . . . . . . 53

$4.5 .6 . \quad$ Trigger System … . . . . . . . . . 55

4.5.7. Scaler Modules . . . . . . . . . . . . . . . . 55

5. Polarized ${ }^{3}$ He Target $\ldots \ldots \ldots \ldots \ldots \ldots \ldots$

5.1. Spin-Exchange Optical Pumping (SEOP) . . . . . . . . . . 57

5.1.1. Optical Pumping . . . . . . . . . . . . . . . . . 58

$5.1 .2 . \quad$ Spin Exchange . . . . . . . . . . . . . . . . . . 59

$5.2 . \quad$ Target Setups $\ldots \ldots \ldots \ldots \ldots$

5.2.1. Characterizing the ${ }^{3} \mathrm{He}$ Cell . . . . . . . . . . . 61

$5.2 .2 . \quad$ Lasers and Optics . . . . . . . . . . . . . . 70

$5.2 .3 . \quad$ Magnet System . . . . . . . . . . . . . . . . 72

5.2.4. Oven, Heater, and Airflow System . . . . . . . . . . 73

$5.2 .5 . \quad$ Target Ladder . . . . . . . . . . . . . . . . 74

5.2.6. Cell Temperature and Density During E05-015 . . . . . . 75

5.3. Nuclear Magnetic Resonance (NMR) Polarimetry . . . . . . . . 78

5.3.1. AFP Process $\ldots \ldots \ldots \ldots \ldots \ldots$

5.3.2. NMR Measurement . . . . . . . . . . . . . . . . . . 79

5.4. Electron Paramagnetic Resonance (EPR) Polarimetry . . . . . . . 82

5.5. Polarization Results from EPR Measurement . . . . . . . . . . . 85

5.6. Calibration of NMR with EPR. . . . . . . . . . . . 85

5.7. Polarization Gradients . . . . . . . . . . . . . . . 85

5.7.1. Two Chamber Polarization Model . . . . . . . . . . . . 87

$5.7 .2 . \quad$ Diffusion Rate $d_{t c} \ldots \ldots \ldots \ldots \ldots$

$5.7 .3 . \quad$ Target Chamber Life Time . . . . . . . . . . . . . . 89

5.7.4. Polarization Gradient Result. . . . . . . . . . . . . . 92 
5.8. Target Performance . . . . . . . . . . . . . . . . . . 93

6. Data Analysis . . . . . . . . . . . . . . . . . . 95

6.1. Asymmetry Extraction . . . . . . . . . . . . . . . . . . . . 97

6.1.1. Raw Asymmetry $A_{\text {raw }} \ldots \ldots \ldots \ldots \ldots$

6.1.2. ${ }^{3} \mathrm{He}$ Experimental Asymmetry $A_{e x p}^{3} \mathrm{He} \ldots \ldots \ldots$. . . . . 97

6.1.3. ${ }^{3} \mathrm{He}$ Experimental Asymmetry in the Quasi-Elastic Region

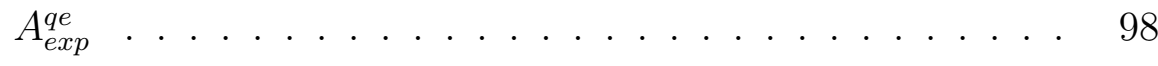

6.1.4. Prescale Factor Correction . . . . . . . . . . . . . . 99

6.2. Scaler Consistency Check . . . . . . . . . . . . . . . . . . 99

6.3. Calibration . . . . . . . . . . . . . . 100

6.3.1. Beam Current Monitor Calibration . . . . . . . . . . . . 100

6.3.2. Optics Reconstruction . . . . . . . . . . . . . . . . . 104

Coordinate Systems . . . . . . . . . . . . . . . . . . 104

Calibrations ..................... 105

6.3.3. Detector Calibration . . . . . . . . . . . . . . 107

6.4. Event Selection . . . . . . . . . . . . . . . . . . . 108

6.5. DAQ Live-Time . . . . . . . . . . . . . . . . . . . . 117

6.6. Data Binning and Kinematics . . . . . . . . . . . . . 117

6.7. Nitrogen and Glass Dilution Factor . . . . . . . . . . . . 117

6.8. Monte Carlo Simulation . . . . . . . . . . . . . . . 120

6.8.1. Radiative Corrections. . . . . . . . . . . . . . . . . 120

6.8.2. Elastic Radiative Tail Dilution Factor . . . . . . . . . . . 124

6.8.3. Quasi-Elastic Asymmetry Radiative Correction . . . . . . 125

6.9. Proton Dilution Factor . . . . . . . . . . . . . . . . . . . . 125

6.10. Systematic Uncertainties . . . . . . . . . . . . . . . . . 128

7. Results and Conclusions . . . . . . . . . . . . . . . . . 133 
7.1. Raw Asymmetry $\left(A_{y}^{\text {raw }}\right)$. . . . . . . . . . . . . . . 134

7.2. ${ }^{3} \mathrm{He}$ Experimental Asymmetry $\left(A_{y}^{3} \mathrm{He}\right)$. . . . . . . . . . . . . . 136

7.3. Combined LHRS and RHRS $A_{y}^{3} \mathrm{He}$. . . . . . . . . . . . . . . . . 140

7.4. ${ }^{3}$ He Experimental Asymmetry in the Quasi-Elastic Region $\left(A_{y}^{\mathrm{qe}}\right)$. 141

7.5. Neutron Asymmetry in the Quasi-Elastic Region $\left(A_{y}^{n}\right)$. . . . . . . 144

7.6. Conclusions and Outlook . . . . . . . . . . . . . . . . . . . . 145

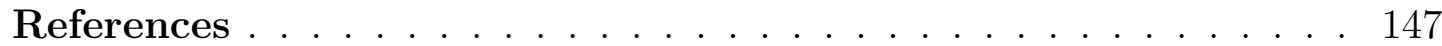

Appendix A. Wall Thickness Measurement Results . . . . . . . . . 151 


\section{List of Tables}

2.1. Important kinematic factors expressed in the lab frame. . . . . . . 12

2.2. Fit parameters from data for nucleon electromagnetic form factors used by J. J. Kelly. . . . . . . . . . . . . . . . . . . . . . . . . . . 18

3.1. Summary of the $A_{y}^{p}$ measurements in the past years. . . . . . . . . 33

4.1. Beam energy from Tiefenback measurements for different settings of E05-015. . . . . . . . . . . . . . . . . . . . . 42

4.2. Main characteristic of the Hall A high resolution spectrometers. . 48

4.3. Triggers used during E05-015. . . . . . . . . . . . . . . . . . . . . 56

5.1. Characteristics of ${ }^{3} \mathrm{He}$ cell used in E05-015. . . . . . . . . . . . . . 62

5.2. Parameters for the pressure broadening measurement. . . . . . . . 64

5.3. Pressure broadening coefficients. . . . . . . . . . . . . . . . . 66

5.4. Laser parameters used throughout the experiment. . . . . . . . . 71

5.5. Basic characteristics and power supplies of Helmholtz coils. . . . . 73

5.6. Current set points for each coil for generating $25 \mathrm{G}$ holding field in vertical direction. . . . . . . . . . . . . . . . . . . . . . . . . . 73

5.7. $\quad$ Parameters used in extracting polarization from EPR signals. . . 83

5.8. Parameters used in $\frac{d \nu_{\mathrm{EPR}}}{d B}$ calculation. . . . . . . . . . . . 85

5.9. The constants used in Equation (5.45). . . . . . . . . . . . . . 90

5.10. Systematic uncertainty budget for the target polarization. . . . . 94

6.1. Summary of the scaler consistency check results. . . . . . . . . . . 101

6.2. The calibration of the u3 BCM with the beam current value from the injector. . . . . . . . . . . . . . . . . . . 102 
6.3. Summary of parameters used for vertex cuts. . . . . . . . . . . . . 114

6.4. Summary of parameters used for pion rejecter/shower cut. . . . . 115

6.5. Summary of the binning of the energy transfer $\omega$ in E05-015. . . . 117

6.6. Summary of the nitrogen and glass dilution factors. . . . . . . . . 119

6.7. Properties of radiation materials before and after scattering. . . . 122

6.8. Summary of the elastic radiative tail dilution factors. . . . . . . . 125

6.9. Radiative correction to quasi-elastic asymmetry. . . . . . . . . . . 126

6.10. Rosenbluth cross-sections for nucleons. . . . . . . . . . . . . . . . 127

6.11. LHRS $A_{y}^{3 \mathrm{He}}$ systematic uncertainty budget at $\mathrm{E}=1.245 \mathrm{GeV}$. . . . 130

6.12. RHRS $A_{y}^{3} \mathrm{He}$ systematic uncertainty budget at $\mathrm{E}=1.245 \mathrm{GeV}$. . . . 130

6.13. LHRS $A_{y}^{3 \mathrm{He}}$ systematic uncertainty budget at $\mathrm{E}=2.425 \mathrm{GeV}$. . . . 131

6.14. RHRS $A_{y}^{3} \mathrm{He}$ systematic uncertainty budget at $\mathrm{E}=2.425 \mathrm{GeV}$. . . . 131

6.15. LHRS $A_{y}^{3 \mathrm{He}}$ systematic uncertainty budget at $\mathrm{E}=3.605 \mathrm{GeV}$. . . . 131

6.16. RHRS $A_{y}^{3 \mathrm{He}}$ systematic uncertainty budget at $\mathrm{E}=3.605 \mathrm{GeV}$. . . . 131

6.17. Combined $A_{y}^{3} \mathrm{He}$ systematic uncertainty budget. . . . . . . . . . . 132

6.18. Neutron asymmetry systematic uncertainty budget. . . . . . . . . 132

7.1. LHRS $A_{y}^{\text {raw }}$ results summary. . . . . . . . . . . . . . . . . . . . . 134

7.2. RHRS $A_{y}^{\text {raw }}$ results summary. . . . . . . . . . . . . . . . 135

7.3. LHRS $A_{y}^{3} \mathrm{He}$ results summary. . . . . . . . . . . . . . . . . . . . . . 136

7.4. RHRS $A_{y}^{3} \mathrm{He}$ results summary. . . . . . . . . . . . . . . . . . . . . 137

7.5. $A_{y}^{3} \mathrm{He}$ results summary. . . . . . . . . . . . . . . . . . . . . . 140

7.6. Summary of Quasi-Elastic $A_{y}^{3} \mathrm{He}$ results for $\mathrm{E}=1.245 \mathrm{GeV}$ data. . . 141

7.7. Summary of results for the neutron asymmetry $A_{y}^{n}$. . . . . . . . . 144

A.1. The reference cell GMB2 wall thickness. . . . . . . . . . . . . . . 152

A.2. The ${ }^{3} \mathrm{He}$ cell Dominic wall thickness. . . . . . . . . . . . . . . . . 152 


\section{List of Figures}

1.1. Length scales and structural hierarchy in atomic structure. . . . . 3

2.1. Description of the differential cross-section. . . . . . . . . . . . . . 8

2.2. One photon exchange Feynman diagram for the electromagnetic interaction. . . . . . . . . . . . . . . . . . . 9

2.3. The complete set of Feynman diagrams to order $\alpha^{2}$. . . . . . . . . 11

2.4. Inclusive cross-section versus $\mathrm{Q}^{2}$ and $\omega$ for scattering off a nuclear target. . . . . . . . . . . . . . . 16

2.5. The $F_{2}^{p}$ structure function $Q^{2}$-dependence for a range of Bjorken $x$ values. . . . . . . . . . . . . . . . . . 21

3.1. Ratio of proton electric form factor to magnetic form factor. . . . 28

3.2. Proton $\mu G_{E} / G_{M}$ ratio obtained from the polarization transfer measurements with two-photon-exchange corrections. . . . . . . . . . 29

3.3. Comparison of polarization transfer measurements and Rosenbluth separations. ........................... 29

3.4. Previous asymmetry measurements from elastic electron-proton scattering. . . . . . . . . . . . . . . . 32

4.1. An aerial view looking southwest at the Jefferson Lab CEBAF accelerator site and three experimental Halls. . . . . . . . . . . . 35

4.2. Sketch of CEBAF . . . . . . . . . . . . . . . . 36

4.3. Experimental Hall A layout during the E05-015. . . . . . . . . . . 39

4.4. The arc section of the beam line. . . . . . . . . . . . . . . . . . . 41

4.5. Schematic of the Hall A beam current monitors. . . . . . . . . . . 44 
4.6. Beam position with beam raster on. . . . . . . . . . . . . . . 46

4.7. HRS layout. . . . . . . . . . . . . . . . . . . . . . . . . . . . . 47

4.8. Illustration of the left and right HRS detector packages. . . . . . . 48

4.9. Top and side view of VDCs . . . . . . . . . . . . . . 50

4.10. Layout of the S1 scintillator counter. . . . . . . . . . . . . . 51

4.11. Cherenkov detector schematic. . . . . . . . . . . . . . . . . 53

4.12. Configuration of electromagnetic calorimeters. . . . . . . . . . . . 54

4.13. HRS single arm trigger scheme. . . . . . . . . . . . . . . . . . 55

5.1. A diagram explaining optical pumping. . . . . . . . . . . . . . . . 58

5.2. Spin exchange in a Rb-K hybrid cell. . . . . . . . . . . . . . . . . 60

5.3. A schematic of the vertically polarized ${ }^{3}$ He target. . . . . . . . . . 61

5.4. Setup for the pressure broadening measurement. . . . . . . . . . . 63

5.5. Illustration of the density scan figure. . . . . . . . . . . . . 65

5.6. Setup for cell wall thickness measurement. . . . . . . . . . . . . . 67

5.7. An example of measured positions in wall thickness measurements. 69

5.8. An example picture of the interference pattern. . . . . . . . . . . 69

5.9. A schematic diagram of the optics setup. . . . . . . . . . . . . . . 72

5.10. Top view of the coil setup for the ${ }^{3} \mathrm{He}$ target. . . . . . . . . . . . . 73

5.11. A front view of the target ladder. . . . . . . . . . . . . . . . . 74

5.12. RTD positions on the ${ }^{3} \mathrm{He}$ target. . . . . . . . . . . . . . . . . 76

5.13. Temperature in the cell during E05-015. . . . . . . . . . . . . . . 77

5.14. Density in the cell during E05-015. . . . . . . . . . . . . . . . . . 77

5.15. A typical NMR frequency sweep signal. . . . . . . . . . . . . . . . 80

5.16. Energy level of ${ }^{85} \mathrm{Rb}$. . . . . . . . . . . . . . . . . 81

5.17. An example of an online EPR measurement. . . . . . . . . . . . . 86

5.18. EPR polarization results from the calibration measurements. . . . 86

5.19. Target spin down curve at room temperature. . . . . . . . . . . . 91 
5.20. Beam depolarization effect. . . . . . . . . . . . . . . . . . 91

5.21. Spin up measurement for the cell Dominic. . . . . . . . . . . . . 92

5.22. Target polarization versus spin flip. . . . . . . . . . . . . . 93

6.1. The data analysis flow chart. . . . . . . . . . . . . . . 96

6.2. The calibration of the $\mathrm{u} 3 \mathrm{BCM}$ with the beam current value from the injector. . . . . . . . . . . . . . . . . . . 103

6.3. A schematic of three coordinate systems in common use in Hall A. 104

6.4. LHRS reconstructed vertex position. . . . . . . . . . . . 106

6.5. The schematic of the sieve pattern used to calibrate the HRS. . . 107

6.6. Spectra for the LHRS Gas Cherenkov detector after calibration. . 109

6.7. Energy deposited in the pion rejecters after calibration. . . . . . . 110

6.8. An example of beam trip cut. . . . . . . . . . . . . . . . . 111

6.9. Left HRS geometry cuts at $\mathrm{E}=1.245 \mathrm{GeV} . \quad \ldots \ldots \ldots$

6.10. An example of track number. . . . . . . . . . . . . . . 114

6.11. An example of the vertex cut. . . . . . . . . . . . . . 115

6.12. Comparison of the data from ${ }^{3} \mathrm{He}$ target cell and empty target cell. 116

6.13. Schematic drawing of materials in particle's path. . . . . . . . . . 121

6.14. Second order Feynman diagrams considered for internal radiation correction. . . . . . . . . . . . . . . . . . 123

6.15. Comparison between data and simulation for both the elastic and

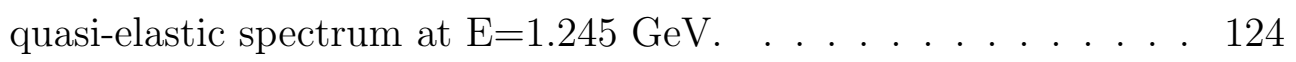

7.1. ${ }^{3} \mathrm{He}$ asymmetries from LHRS and RHRS plotted versus energy transfer $\omega . \ldots \ldots \ldots \ldots$

7.2. ${ }^{3} \mathrm{He}$ asymmetries from LHRS and RHRS as a function of 4-momentum transfer squared $\left(Q^{2}\right)$.

7.3. ${ }^{3} \mathrm{He}$ asymmetries in the quasi-elastic region are shown versus energy transfer $\omega . \ldots \ldots \ldots . \ldots \ldots$ 
7.4. ${ }^{3}$ He asymmetries summed over the quasi-elastic region plotted versus 4 -momentum transfer squared $\left(Q^{2}\right)$. . . . . . . . . . . . . . . 143

7.5. The neutron asymmetry $A_{y}^{n}$ as a function of 4-momentum transfer squared $\left(Q^{2}\right) . \ldots \ldots \ldots \ldots \ldots$. . . . . . . . . . . . 144

7.6. Neutron asymmetries of this work compared with the available proton asymmetry results. . . . . . . . . . . . . . . . . . . . . 146 


\section{Chapter 1 \\ Introduction}

In the field of science and philosophy, humans have sought answers to the most fundamental questions: What are the fundamental building blocks of matter, and how do they build our universe? To answer these questions, people have come a long way, and how these questions were answered illustrates the thinking process of scientists and philosophers. In this process, people have learned about our universe, the composition of materials, and their various properties. However, regarding the internal structure of matter, there are still very important challenges that need to be resolved.

\subsection{Fundamental Constituents of Matter}

Physicists have discovered smaller and smaller constituents in the search for the basic building blocks of matter. By the end of $19^{\text {th }}$ century, it was generally acknowledged that all substances are composed of atoms, and that atoms are not only the building blocks of matter, but they are also inseparable. In 1897, British scientist J. J. Thomson discovered the negatively charged electron, which led to the idea that atoms have an internal structure [2]. Thomson proposed the Plum Pudding Model [3], which postulated that atomic structure is similar to one of the most favorite English dessert - plum pudding. Negatively charged electrons are immersed in a sea of positive charge, which is similar to the raisins dispersed among plum pudding.

Nearly a decade after Thomson proposed his plum pudding model, Ernest 
Rutherford proposed the Rutherford Model [4] based on his famous gold foil experiments results. Rutherford's model suggested that the atom consists of a nucleus and electrons, where the nucleus is a densely packed core of positive charge and is surrounded by a negatively charged electron cloud. In 1919, using alpha particles as projectiles, Rutherford knocked hydrogen nuclei out of atoms of 6 elements: boron, fluorine, sodium, aluminum, phosphorus, and nitrogen. He named them protons and predicted the existence of uncharged neutrons, which was verified by James Chadwick in 1932 [5]. The protons and neutrons constitute the nucleus and are commonly called nucleons.

Particle accelerator experiments in the 1950's and 60's showed that protons and neutrons are part of a larger family of particles called hadrons. Today, more than 100 hadrons have been discovered. Initially, physicists thought that all hadrons are point-like particles, and they do not have internal structure. However, it was soon realized that this was not the case. In order to explain hadrons, it has been speculated [6] [7] that the hadrons are composed of point-like particles called quarks. Quarks have spin-1/2, fractional charge, and a new degree of freedom - flavor. At present, physicists know of two basic types of building blocks: quarks and leptons. We know there are 6 leptons and 6 quarks as well as their antiparticles. Figure 1.1 shows the different scales of the structure of matter; with increasing magnification of the probe, smaller and smaller structures become visible: the nucleus, the nucleons, and finally the quarks.

\subsection{Fundamental Interactions}

Four fundamental interactions have been identified in our universe: the strong interaction, electromagnetic interaction, weak interaction and gravitational interaction [8]. The properties of the four interactions are quite different:

- The strong interaction, or strong nuclear force, is very strong, but only over 


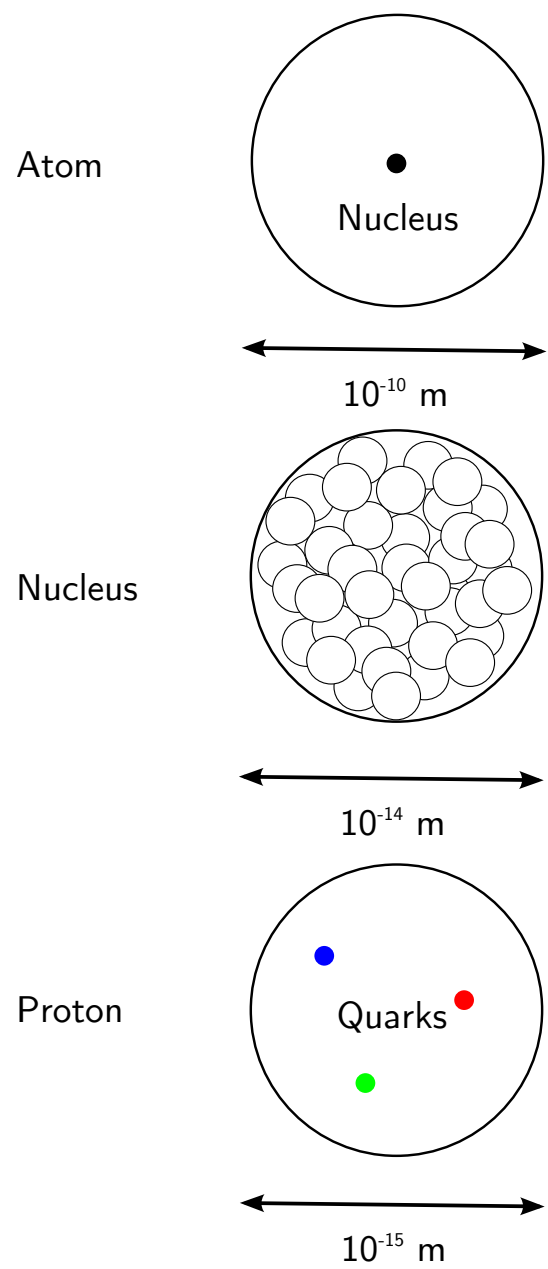

Figure 1.1: Length scales and structural hierarchy in atomic structure. 
very short-range. Its interaction range is of the order of $10^{-15} \mathrm{~m}$, it exists only inside of the atomic nucleus and is responsible for holding hadrons and the nuclei of atoms together. The strong interaction is mediated by gluons.

- The electromagnetic interaction causes electric and magnetic effects such as the repulsion between similar electrical charges or the interaction of bar magnets. The force is long-ranged, but the interaction force is much weaker than the strong force. It acts only between pieces of matter carrying electrical charge. The electromagnetic force is mediated by photons.

- The weak interaction is weak and has a short range. Its interaction range is about $10^{-18} \mathrm{~m}$ and it is responsible for radioactive decays and neutrino interactions. The weak force is mediated by the $W^{+}, W^{-}$and $Z^{0}$.

- The gravitational interaction force is weak and the interaction range is infinite. In addition, this force is always attractive and acts on all particles having mass in our unverse. The gravitational force is mediated by gravitons - but it is the only force for which we do not have a firmly established quantum theory.

\subsection{Why Electron Scattering}

There are many reasons for considering electron-nucleus scattering as a method for exploring the structure of the nucleus and nucleons [9]:

- The electron interacts with the nucleus by means of the electromagnetic force. The electromagnetic interaction is calculable with the well understood theory of Quantum Electro-Dynamics (QED) [10]. One can extract detailed information about the nuclear structure by probing the nuclear current $J_{\mu}$. On the other hand, protons and pions interact with the nucleus by means of 
the strong force, and hence, one must rely on phenomenological models to understand the interaction at lower energies and interpret nuclear structure.

- The electromagnetic force is relatively weak compared to the strong force. The corresponding coupling constant, $\alpha \simeq 1 / 137$, is less than the characteristic strength of the strong force, which is responsible for the major part of nuclear properties. This means that electron scattering measurements do not greatly perturb the structure of the nucleus. On the other hand, proton and pion scattering are dominated by the strong force, which requires separation of the reaction mechanisms from the appropriate nuclear structure problem.

- The interaction between the electron and the nucleus is carried out by means of a virtual photon with 4 -momentum $q=(\omega, \vec{q})$, and Lorentz invariant $Q^{2}=-q^{2}=\vec{q}^{2}-\omega^{2} \geq q^{1}$. The energy and 3-momentum can be varied independently. For example, one can fix the energy transfer $\omega$ and map out the spatial distributions of the nuclear charge and current densities by measuring the nuclear responses at different $\vec{q}$.

\subsection{Outline of the Dissertation}

This dissertation is organized into seven chapters. Chapter 1 gives a brief history of particles and basic interactions and explains the advantage of electron scattering experiments. Chapter 2 describes the general formalism and different inclusive scattering processes. Chapter 3 discusses the theoretical motivation for the measurement as well as the available world data. Chapter 4 describes the equipment used throughout the experiment. This includes both information about the electron beam and the equipment within Jefferson Lab's experimental

\footnotetext{
${ }^{1}$ Note that for real photon absorption $Q^{2}=-q^{2}=\vec{q}^{2}-\omega^{2}=0$.
} 
Hall A except the target. Chapter 5 introduces the polarized ${ }^{3} \mathrm{He}$ target at Jefferson Lab. The methods used for extracting the physics results are described in Chapter 6, including detector calibrations, particle identification and various corrections. Finally, the results from the measurements are presented in Chapter 7. 


\section{Chapter 2}

\section{General Formalism}

This chapter will briefly describe the theory of electron scattering. First, the kinematic variables will be defined. Then starting from Rutherford's basic formula, the necessary pieces will be added on to make it applicable to electron scattering from a nucleus. The process of inclusive electron-nucleon scattering, where only the scattered electron is detected, will be discussed. The types of inclusive electron scattering and the differential cross-section will be presented.

\subsection{Cross-Section}

The most important quantity for the description and interpretation of scattering experiments is the cross-section.

From theory: Following [11], in a reaction, the interaction potential, which is described by the Hamilton operator $\mathcal{H}_{\text {int }}$, transforms the initial-state wave function $\psi_{i}$ into the final-state wave function $\psi_{f}$, and the transition matrix element is given by:

$$
\mathcal{M}_{f i}=\left\langle\psi_{f}\left|\mathcal{H}_{i n t}\right| \psi_{i}\right\rangle=\int \psi_{f}^{*} \mathcal{H}_{i n t} \psi_{i} d V
$$

Using this transition matrix element, the cross-section can be written as:

$$
\sigma=\frac{2 \pi}{\hbar \cdot v}\left|\mathcal{M}_{f i}\right|^{2} \cdot \rho\left(E^{\prime}\right) \cdot V
$$

where $v$ is the particle velocity before scattering, $V$ is the spatial volume occupied by the beam particles, and $\rho\left(E^{\prime}\right)$ is the density of the final states. 


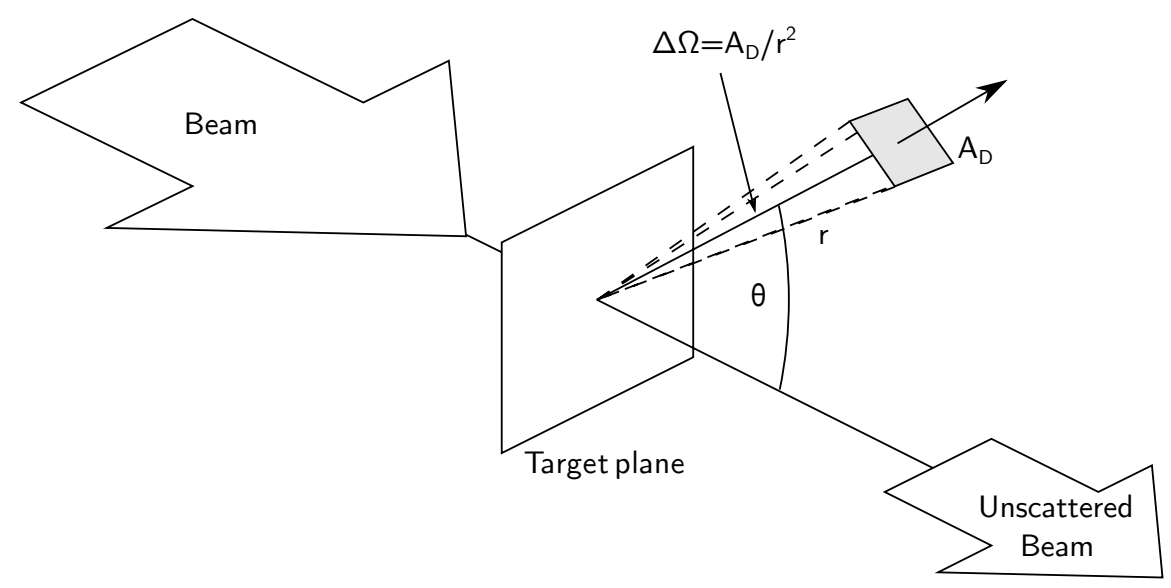

Figure 2.1: Description of the differential cross-section. Only particles scattered into the small solid angle $\Delta \Omega$ are recorded by the detector of cross-sectional area $A_{D}$.

One can calculate the cross-section from Equation $(2.2)$ if the interaction potential is known. On the other hand, the transition matrix elements can be determined by Equation (2.2) if the cross-section is known.

From experiment: As shown in Figure 2.1, for a detector at an angle $\theta$ with respect to the incident beam, the number of particles per unit area hitting the detector is given by the differential cross-section, which is defined as: $\frac{d \sigma(E, \theta)}{d \Omega}=\frac{\text { number of particles scattered into solid angle } \mathrm{d} \Omega \text { per unit time }}{\text { luminosity }}$.

The total cross-section $\sigma$ can then be obtained by integral over the total solid angle:

$$
\sigma_{\text {tot }}(E)=\int_{4 \pi} \frac{d \sigma(E, \theta)}{d \Omega} d \Omega .
$$

\section{$2.2 \quad$ Feynman Diagram}

Richard Feynman developed a relatively simple method to calculate the matrix element $\mathcal{M}[12$. He suggested that one can simply draw a stick figure diagram 


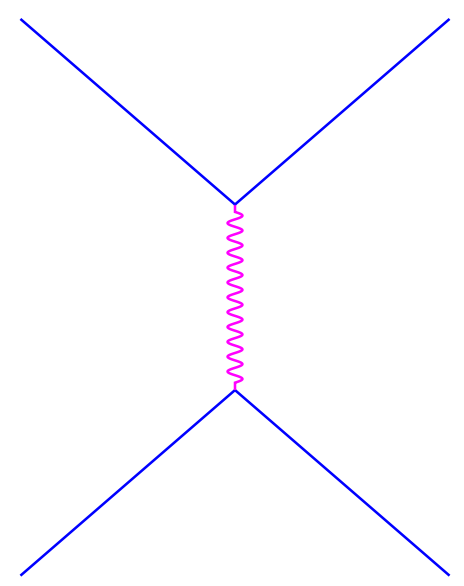

Figure 2.2: One photon exchange Feynman diagram for the electromagnetic interaction.

to represent the scattering process. The full matrix element is represented by the sum of all possible diagrams between the initial and final states, but for interactions which are weak, one only needs a small set of diagrams with the least complexity and fewest interactions. In a Feynman diagram, each symbol corresponds to a factor in the matrix element. The links between the individual terms and the meaning of them are fixed by the Feynman rules. For instance, two electrons interacting via the electromagnetic force are represented in Figure 2.2. For this thesis, the diagram is read with time flowing from left to right. The straight lines in the graph correspond to incoming or outgoing electrons and wavy line stands for the photon. The interaction process corresponds to a photon being emitted by one electron and absorbed by the other electron. This photon is referred to as a virtual photon because it is neither in the initial nor in the final state. Points where particles meet are called vertices, and energy and momentum are conserved at each vertex. In Figure 2.2, the exchanged photon couples to the charge of one electron at the top vertex and to that of the other electron at the bottom vertex. For each vertex, the transition amplitude contains a factor which is proportional to $e$, i.e., $\sqrt{\alpha}$. 
Figure 2.2 shows the basic means by which two electrons can interact. However, to fully describe an interaction process, even for a simple interaction like electron-electron scattering, an infinite number of diagrams are in principle needed. The complete set of diagrams to order $\alpha^{2}$ are shown in Figure 2.3. In QED, this problem has a solution: the coupling constant corresponding to each photon is $\alpha$, as more and more vertices are present in a Feynman diagram, the Feynman diagram contributes less and less to the over all cross-section. A cut off must be made with regard to the number of Feynman diagrams to include if one wants to use Feynman's procedure to calculate a cross-section. In many cases, one only needs the very first Feynman diagram. It is sufficient because the next-to-leadingorder diagrams include an extra factor of $\alpha$ in the cross-section, and thus these diagrams contribute at a level that is typically several orders of magnitude smaller to the overall cross-section.

\subsection{Kinematic Variables}

In inclusive electron scattering experiments, the structure of a target nucleon or nucleus is studied with the differential cross-section in the process

$$
l(k)+N(p) \rightarrow l\left(k^{\prime}\right)+X\left(p^{\prime}\right)
$$

where $l(k)$ is an electron with 4-momentum $k, N(p)$ is a nucleon or nucleus with 4-momentum $p$ and $X\left(p^{\prime}\right)$ is the hadronic final state with 4-momentum $p^{\prime}$. Before writing the cross-section for this process, it is useful to describe some basic variables. Table 2.1 is a list of the most common kinematic variables used in parameterizing electron scattering. 


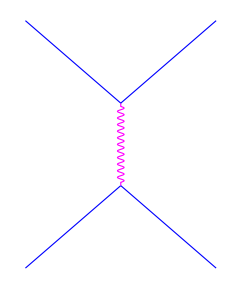

(a)

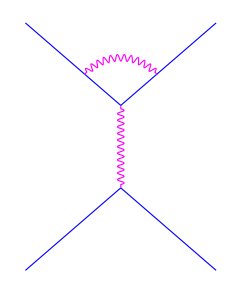

(e)

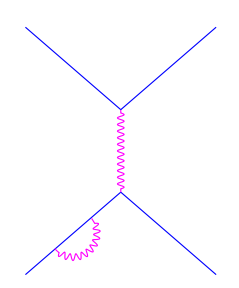

(i)

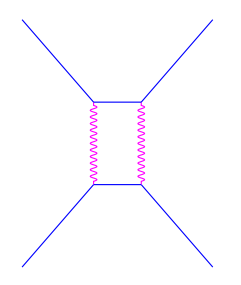

(b)

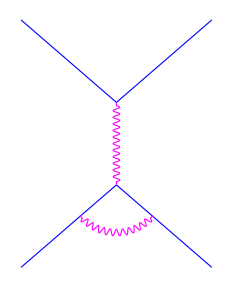

(f)

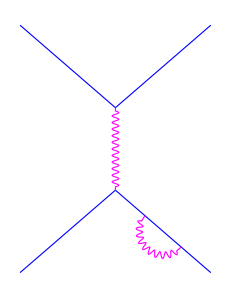

(j)

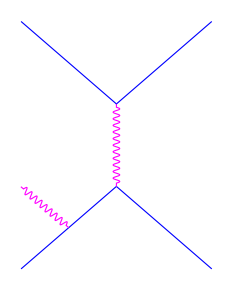

$(\mathrm{m})$

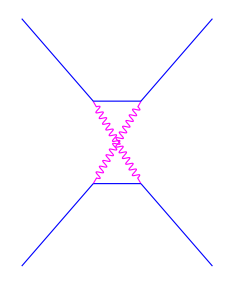

(c)

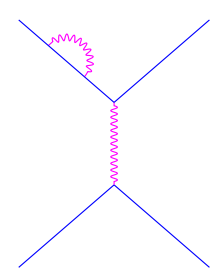

$(\mathrm{g})$

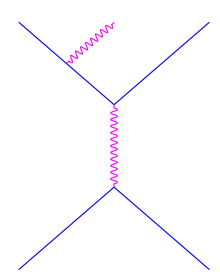

(k)

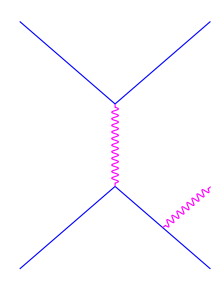

(n)

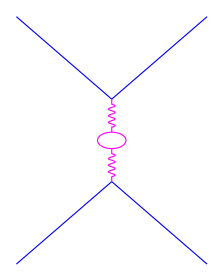

(d)

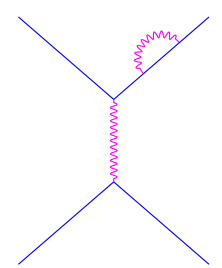

(h)

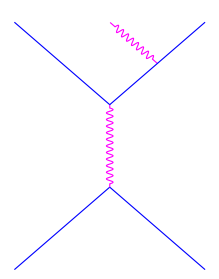

(l)

Figure 2.3: The complete set of Feynman diagrams to order $\alpha^{2}$. (a) elastic electron Born diagram scattering. (b) box diagram. (c) crossed-box diagram. (d) vacuum polarization. (e-f) electron vertex corrections. ( $g-j)$ electron self-energy diagrams. $(\mathrm{k}-\mathrm{n})$ inelastic Bremsstrahlung amplitudes. 
Table 2.1: Important kinematic factors expressed in the lab frame. The mass of the electron $m_{e}$ is neglected, a fixed target is assumed, and units with $\hbar=c=1$ are adopted.

\begin{tabular}{|c|c|}
\hline Parameter & Description \\
\hline$E$ & incident electron energy \\
$p=(M, 0,0,0)$ & target 4-momentum \\
$(\theta, \phi)$ & (polar, azimuthal) scattering angle \\
$E^{\prime}$ & scattered electron energy \\
$\omega=E-E^{\prime}$ & energy transfer \\
$k=(E, \vec{k})$ & incident electron 4-momentum \\
$k^{\prime}=\left(E^{\prime}, \overrightarrow{k^{\prime}}\right)$ & scattered electron 4-momentum \\
$\vec{q}=\vec{k} \cdot \overrightarrow{k^{\prime}}$ & 3-momentum transfer \\
$q=k-k^{\prime}$ & 4-momentum transfer \\
$Q^{2}=-q^{2} \approx 4 E E^{\prime} \sin ^{2} \frac{\theta}{2}$ & 4-momentum transfer squared, assuming relativistic electrons \\
$W^{2}=(p+q)^{2}=M^{2}+2 M \omega-Q^{2}$ & squared invariant mass of the final hadronic system \\
$x=\frac{Q^{2}}{2 M \omega}=\frac{Q^{2}}{2 p \cdot q}$ & Bjorken scaling variable \\
$y=\frac{q \cdot p}{k \cdot p}=\frac{\omega}{E}$ & fractional energy transfer \\
\hline
\end{tabular}

\subsection{The Rutherford and Mott Cross-Sections}

Considering the case of an electron with energy $E$ scattering off an atomic nucleus with charge $Z e$, the cross-section can be calculated with the Rutherford crosssection formula1,

$$
\left(\frac{d \sigma}{d \Omega}\right)_{\text {Rutherford }}=\frac{4 Z^{2} \alpha^{2} E^{\prime 2}}{|\vec{q}|^{4}}
$$

where $\vec{q}$ is the 3 -momentum transfer, $\alpha$ is the fine structure constant and $E^{\prime}$ is the energy of the scattered electron. The Rutherford formula ignores nuclear structure and recoil, and spins of the electron and the target, so the formula is applied to heavy nuclei and low-energy electrons. The Rutherford cross-section formula can be modified to include spin effects. The Mott cross-section [13], which describes the electron scattering and includes effects due to the electron spin, is written as:

$$
\left(\frac{d \sigma}{d \Omega}\right)_{\text {Mott }}^{*}=\left(\frac{d \sigma}{d \Omega}\right)_{\text {Rutherford }} \cdot \cos ^{2} \frac{\theta}{2} .
$$

\footnotetext{
${ }^{1}$ We set $\hbar=c=1$, and neglect the mass $m_{e}$ of the electron.
} 
The factor $\cos ^{2}(\theta / 2)$ prevents scattering at $180^{\circ}$ from a spin-less target, thus conserving helicity [11]. The $*$ indicates that we are still ignoring target recoil due to the mass of heavy nuclei.

For electron scattering from a nucleus, the energy and momentum transferred are absorbed by the recoil nucleus. For high energy electron scattering, the target recoil can no longer be neglected. If we take the target recoil into account, we find an additional factor of $E^{\prime} / E$ in the Mott cross-section:

$$
\left(\frac{d \sigma}{d \Omega}\right)_{\mathrm{Mott}}=\left(\frac{d \sigma}{d \Omega}\right)_{\mathrm{Mott}}^{*} \cdot \frac{E^{\prime}}{E} .
$$

Since the energy loss of the electron due to the recoil is now significant, it is no longer possible to describe the scattering in terms of a 3-momentum transfer. In cross-section formula, $\vec{q}^{2}$ must be replaced by the 4 -momentum transfer squared, $Q^{2}$.

\subsection{Nuclear Form Factors}

The Mott cross-section formula can be used to estimate the cross-section for electron scattering from a point-like target with no internal structure. In scattering experiments with nuclei or nucleons, the cross-section from the Mott formula agrees with the experimental result only at limit of $Q^{2} \rightarrow 0$. At large $Q^{2}$ values, the experimental results are smaller than the cross-sections obtained from the Mott formula due to the reduction of the virtual photon's wavelength at large $Q^{2}$ values. This means that the resolution of the probe increases and that the virtual photon can no longer probes the nuclei or nucleons as a whole, but it can resolve the internal structure. The spatial extent of a nucleus is described by a form factor, and the form factor only depends on the momentum transfer $Q^{2}$. Taking into account the spatial extent of the nuclei, the cross-section becomes [11]:

$$
\left(\frac{d \sigma}{d \Omega}\right)_{\exp }=\left(\frac{d \sigma}{d \Omega}\right)_{\mathrm{Mott}}^{*} \cdot\left|F\left(Q^{2}\right)\right|^{2}
$$


where $F\left(Q^{2}\right)$ is the nuclear form factor and measures the deviation of the target nucleus from point-like behavior. For a point-like charge, the form factor would be a constant.

If we define a charge distribution function $f$ by $\rho(r)=Z e f(r)$ which satisfies the normalization condition $\int f(r) d^{3} r=1$, the matrix element in equation 2.1 becomes [1]:

$$
\begin{aligned}
\mathcal{M}_{f i} & =\left\langle\varphi_{f}\left|\mathcal{H}_{i n t}\right| \varphi_{i}\right\rangle \\
& =\int \varphi_{f}^{*} \mathcal{H}_{i n t} \varphi_{i} d V \\
& =\frac{Z \cdot 4 \pi \alpha \hbar^{3} c}{|\vec{q}|^{2} \cdot V} \int f(r) e^{i \vec{q} r / \hbar} d^{3} r .
\end{aligned}
$$

The integral

$$
F\left(Q^{2}\right)=\int f(r) e^{i \vec{q} r / \hbar} d^{3} r
$$

is the Fourier transform of the charge function $f(r)$, normalized to the total charge. It is the form factor of the charge distribution. All information related to the charge of the objects being studied is contained in this form factor, since in principle, the charge distribution $f(r)$ can be determined from the inverse Fourier transform by:

$$
f(r)=\frac{1}{(2 \pi)^{3}} \int F\left(Q^{2}\right) e^{-i \vec{q} r / \hbar} d^{3} q .
$$

Besides the interaction of the electron with the nucleon charge, we also need to consider the interaction between the electron current and the nucleon's magnetic moment [14]. Two form factors are needed to characterize both the electric and magnetic distributions of spin-1/2 nucleons: the electric form factor and the magnetic form factor.

The form factors can also be viewed as the distributions of charge and magnetization in momentum space. This is important as for relativistic systems there are ambiguities in trying to determine the rest-frame charge distribution. The 
ambiguities only affect the Fourier transform, they do not affect the formulas relating the cross-section to the form factors.

\subsection{Types of Inclusive Electron Scattering}

Only the scattered electrons are detected in inclusive electron scattering, which means the scattered electrons are detected from all possible interactions. In exclusive or semi-inclusive scattering, one detects the scattered electron along with some or all of the other particles produced, which allows one to separate out specific reaction channels. This thesis focuses only on inclusive scattering. In this section, three types of scattering will be discussed: elastic, quasi-elastic and inelastic. Inelastic scattering includes both resonance production and deep inelastic scattering. Figure 2.4 shows a typical cross-section spectrum for inclusive scattering from a light nuclear target. The mass of the nucleus and nucleon are given by $M_{T}$ and $M$, respectively. If the target is a nucleon, quasi-elastic scattering does not exist.

\subsubsection{Elastic Scattering off Nucleons}

The cross-section for the scattering of an electron from a nucleon is described by the Rosenbluth formula [16]:

$$
\left(\frac{d \sigma}{d \Omega}\right)=\left(\frac{d \sigma}{d \Omega}\right)_{\mathrm{Mott}} \cdot\left[\frac{G_{E}^{2}+\tau G_{M}^{2}}{1+\tau}+2 \tau G_{M}^{2} \tan ^{2} \frac{\theta}{2}\right],
$$

with $\tau=Q^{2} /\left(4 M^{2}\right)$. Here $G_{E}$ and $G_{M}$ are called the Sachs electric and magnetic form factors, both of which depend upon $Q^{2}$. They are defined in terms of the $\operatorname{Dirac}\left(F_{1}\right)$ and Pauli form factors $\left(F_{2}\right)$ as

$$
\begin{aligned}
& G_{E}\left(Q^{2}\right)=F_{1}\left(Q^{2}\right)-\tau F_{2}\left(Q^{2}\right) \\
& G_{M}\left(Q^{2}\right)=F_{1}\left(Q^{2}\right)+F_{2}\left(Q^{2}\right) .
\end{aligned}
$$




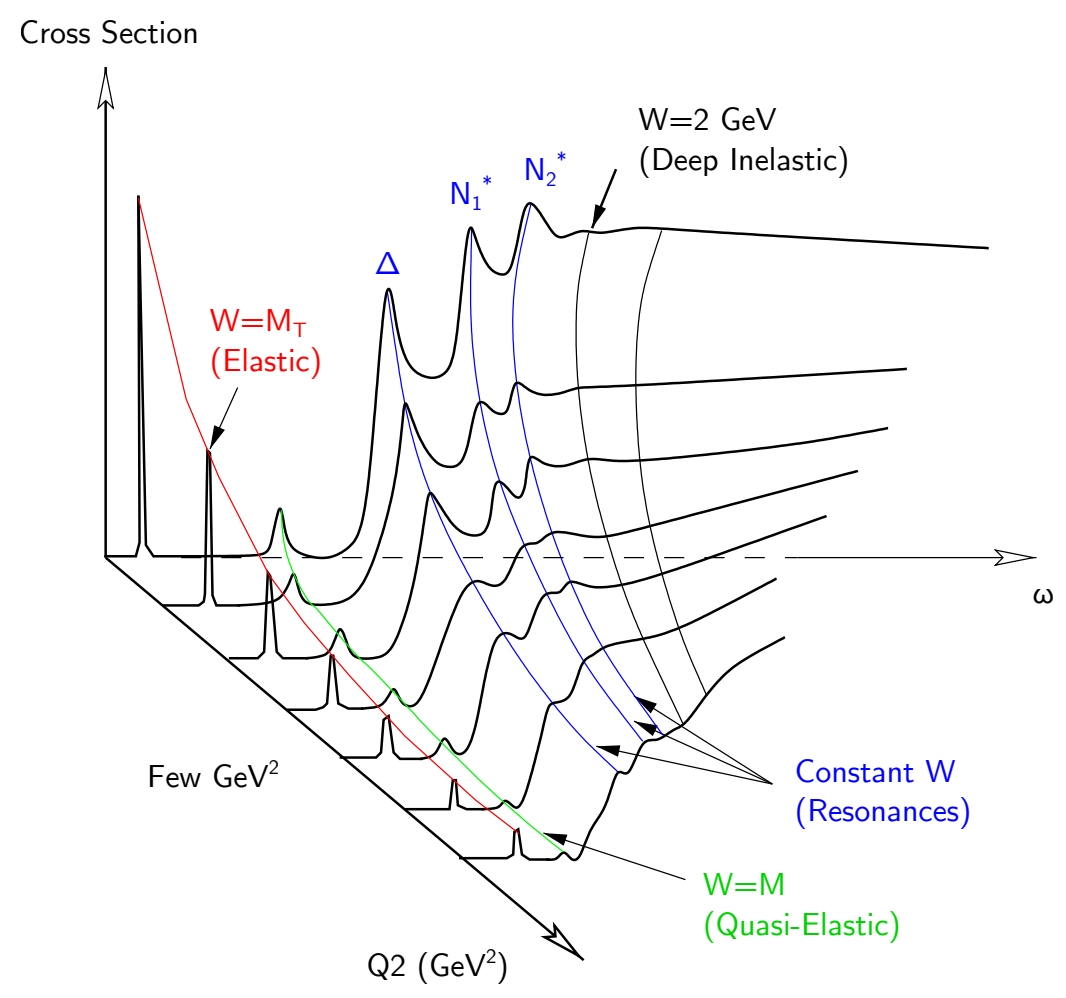

Figure 2.4: Inclusive cross-section versus $\mathrm{Q}^{2}$ and $\omega$ for scattering off a nuclear target [15].

The measured $Q^{2}$-dependence of the form factors gives us information about the charge and magnetic distributions. For elastic scattering, the invariant mass $W$ is equal to the mass of the nucleon so that $\omega=Q^{2} /(2 M)$. Conservation of energy and momentum constrain the scattered electron energy to the following:

$$
E^{\prime}=\frac{E}{1+\frac{2 E}{M} \sin ^{2} \frac{\theta}{2}} .
$$

At $\mathrm{Q}^{2}=0, G_{E}$ coincides with the electric charge of the target, normalized to the elementary charge $e$; and $G_{M}$ is equal to the magnetic moment of the target, normalized to the nuclear magneton $\mu_{N}$. The form factors are given by:

$$
\begin{gathered}
G_{E}^{p}(0)=1 \\
G_{M}^{p}(0)=\mu_{p}=2.793
\end{gathered}
$$

for the proton, and:

$$
G_{E}^{n}(0)=0
$$




$$
G_{M}^{n}(0)=\mu_{n}=-1.913
$$

for the neutron.

In order to independently determine $G_{E}\left(Q^{2}\right)$ and $G_{M}\left(Q^{2}\right)$, the cross-section must be measured at fixed values of $Q^{2}$, for various scattering angles with different beam energies. The measured cross-sections are then divided by the Mott crosssection. If we display the results as a function of $\epsilon=\left[1+2(1+\tau) \tan ^{2}(\theta / 2)\right]^{-1}$, then $G_{E}\left(Q^{2}\right)$ is determined by the slope of the line, and the intercept yields $\tau G_{M}\left(Q^{2}\right)$. If we perform this analysis for various values of $Q^{2}$, we can obtain the $Q^{2}$ dependence of the form factors.

At low $Q^{2}\left(Q^{2} \leq 2 \mathrm{GeV}^{2}\right)$, the proton electric and magnetic form factors, along with neutron magnetic form factor, can be approximately described by a dipole fit [11]:

$$
G_{E}^{p}\left(Q^{2}\right)=\frac{G_{M}^{p}\left(Q^{2}\right)}{\mu_{p}}=\frac{G_{M}^{n}\left(Q^{2}\right)}{\mu_{n}}=G_{D}\left(Q^{2}\right)
$$

where

$$
G_{D}\left(Q^{2}\right)=\left(1+\frac{Q^{2}}{0.71 \mathrm{GeV}^{2}}\right)^{-2}
$$

The electric and magnetic form factors can be more precisely described by J. J. Kelly's fits even at high $Q^{2}$ [17]. Kelly proposed a simple parametrization that is suitable over a wide range, with only four parameters needed for each of $G_{E}^{p}$, $G_{M}^{p}$ and $G_{M}^{n}$. The fits take the form

$$
G\left(Q^{2}\right) \propto \frac{\sum_{k=0}^{n} a_{k} \tau^{k}}{1+\sum_{k=1}^{n+2} b_{k} \tau^{k}},
$$

where $\tau=Q^{2} / 4 M$. Only two parameters are needed for $G_{E}^{n}$ :

$$
G_{E}^{n}=\frac{A \tau}{1+B \tau} G_{D}\left(Q^{2}\right)
$$

The parameters determined from the fits are listed in Table 2.2 . 
Table 2.2: Fit parameters from data for nucleon electromagnetic form factors used by J. J. Kelly [17].

\begin{tabular}{|c|ccccc|cc|}
\hline Quantity & $\mathrm{a}_{0}$ & $\mathrm{a}_{1}$ & $\mathrm{~b}_{1}$ & $\mathrm{~b}_{2}$ & $\mathrm{~b}_{3}$ & $\mathrm{~A}$ & $\mathrm{~B}$ \\
\hline $\mathrm{G}_{E}^{p}$ & 1 & -0.24 & 10.98 & 12.82 & 21.97 & - & - \\
$\mathrm{G}_{M}^{p} / \mu_{p}$ & 1 & 0.12 & 10.97 & 18.86 & 6.55 & - & - \\
$\mathrm{G}_{M}^{n} / \mu_{n}$ & 1 & 2.33 & 14.72 & 24.20 & 84.1 & - & - \\
$\mathrm{G}_{E}^{n}$ & - & - & - & - & - & 1.70 & 3.30 \\
\hline
\end{tabular}

\subsubsection{Quasi-Elastic Scattering}

For a nuclear target, quasi-elastic scattering involves the incident electron elastically scattering from one of the nucleons within the nucleus. In this process, the nucleon is knocked out of the nucleus and can be considered initially as quasifree. Compared to elastic scattering from a free nucleon, the quasi-elastic peak is shifted due to the nuclear binding energy and broadened by the Fermi motion of the nucleons within the nucleus.

The impulse approximation assumes that the electron interacts with a single nucleon, and the nucleon is knocked out of the nuclear system by the scattering process without any further interactions with the remaining nucleons in the nucleus [18]. The shift of the peak is due to the energy required to remove the nucleon from the nucleus. The nucleus is not a static object with locally fixed nucleons, and hence the nucleons move around "quasi-freely" within the nucleus. This motion causes a change in the kinematics compared to scattering off a nucleon at rest and makes the quasi-elastic peak broad.

The Rosenbluth formula is generalized to the form:

$$
\left(\frac{d^{2} \sigma}{d \Omega d E^{\prime}}\right)=\left(\frac{d \sigma}{d \Omega}\right)_{\mathrm{Mott}}\left\{\left(\frac{Q^{2}}{\vec{q}^{2}}\right)^{2} R_{L}+\left[\frac{1}{2}\left(\frac{Q^{2}}{\vec{q}^{2}}\right)^{2}+\tan ^{2} \frac{\theta}{2}\right] R_{T}\right\}
$$

where $\mathrm{R}_{L}\left(\omega, Q^{2}\right)$ and $\mathrm{R}_{T}\left(\omega, Q^{2}\right)$ are the longitudinal and transverse virtual photon response functions. 


\subsubsection{Resonances}

Increasing the energy transfer, we leave the quasi-elastic region and encounter inelastic scattering processes. The resonance region is the first region which is considered to be inelastic with respect to the nucleon. Quarks within the nucleons begin to absorb virtual photons in this region, which may lead the nucleon being excited to a higher resonant state. The presence of these excited states clearly shows that the nucleon is a composite system. The invariant mass of these states is $W^{2}=M^{2}+2 M \omega-Q^{2}$. For elastic scattering $W=M$, so

$$
2 M \omega-Q^{2}=0
$$

while in inelastic processes $W>M$, and

$$
2 M \omega-Q^{2}>0
$$

This suggests the introduction of a dimensionless parameter

$$
x=\frac{Q^{2}}{2 M \omega},
$$

known as the Bjorken variable which measures the inelasticity of the process. For elastic process $x=1$, while for all other processes $0<x<1$. The resonances are observed in the invariant mass region between the pion production threshold $\left(W_{\pi}=M_{p}+m_{\pi}=1.072 \mathrm{GeV}\right)$ and the onset of deep inelastic scattering at $2 \mathrm{GeV}$.

\subsubsection{Deep Inelastic Scattering}

Deep inelastic scattering (DIS) is usually defined as $Q^{2}>1-2 \mathrm{GeV}^{2}$ and $W>$ $2 \mathrm{GeV}$. In this region, the resonance peaks become difficult to distinguish from each other. The DIS process can be seen as the basic process of the quasielastic scattering from the quarks inside the nucleons. The Rosenbluth formula for inelastic scattering is:

$$
\left(\frac{d \sigma}{d^{2} \Omega d E^{\prime}}\right)=\left(\frac{d \sigma}{d \Omega}\right)_{\mathrm{Mott}}^{*}\left[W_{2}\left(\omega, Q^{2}\right)+2 W_{1}\left(\omega, Q^{2}\right) \tan ^{2}\left(\frac{\theta}{2}\right)\right],
$$


where $W_{1}$ and $W_{2}$ are known as structure functions and parameterize all information concerning the target's unknown internal structure. $W_{1}$ and $W_{2}$ are usually replaced by the two dimensionless structure functions:

$$
\begin{aligned}
& F_{1}\left(x, Q^{2}\right)=M W_{1}\left(\omega, Q^{2}\right) \\
& F_{2}\left(x, Q^{2}\right)=\omega W_{2}\left(\omega, Q^{2}\right) .
\end{aligned}
$$

At large $Q^{2}$, the structure functions depend weakly on $Q^{2}$ at fixed values of $x$. This is shown in Figure 2.5. This can be explained as the electrons are scattering from point-like constituents (quarks) inside the nucleon.

\subsection{Tensor Formulation}

In the electron-nucleon scattering process, the inclusive cross-section is proportional to the product of a leptonic and a hadronic tensor, $L_{\mu \nu}$ and $W^{\mu \nu}$, respectively [20]:

$$
\left(\frac{d^{2} \sigma}{d \Omega d E^{\prime}}\right)=\frac{\alpha^{2}}{2 M Q^{4}} \frac{E^{\prime}}{E} L_{\mu \nu} W^{\mu \nu}
$$

The leptonic tensor and hadronic tensor can be further split into symmetric (S) parts and anti-symmetric (A) parts under parity transformation:

$$
\begin{gathered}
L_{\mu \nu}\left(k, s ; k^{\prime}\right)=L_{\mu \nu}^{S}\left(k, k^{\prime}\right)+i L_{\mu \nu}^{A}\left(k, s ; k^{\prime}\right) \\
W_{\mu \nu}(q ; p, S)=W_{\mu \nu}^{S}(q ; p)+i W_{\mu \nu}^{A}(q ; p, S),
\end{gathered}
$$

where $s$ is the spin vector of the electron and $S$ is the spin of the target nucleon. The spin-independent symmetric part and the spin-dependent anti-symmetric parts of lepton tensor are written as

$$
L_{\mu \nu}^{S}\left(k ; k^{\prime}\right)=2\left(k_{\mu} k_{\nu}^{\prime}+k_{\nu} k_{\mu}^{\prime}-g_{\mu \nu}\left(k \cdot k^{\prime}-m^{2}\right)\right)
$$




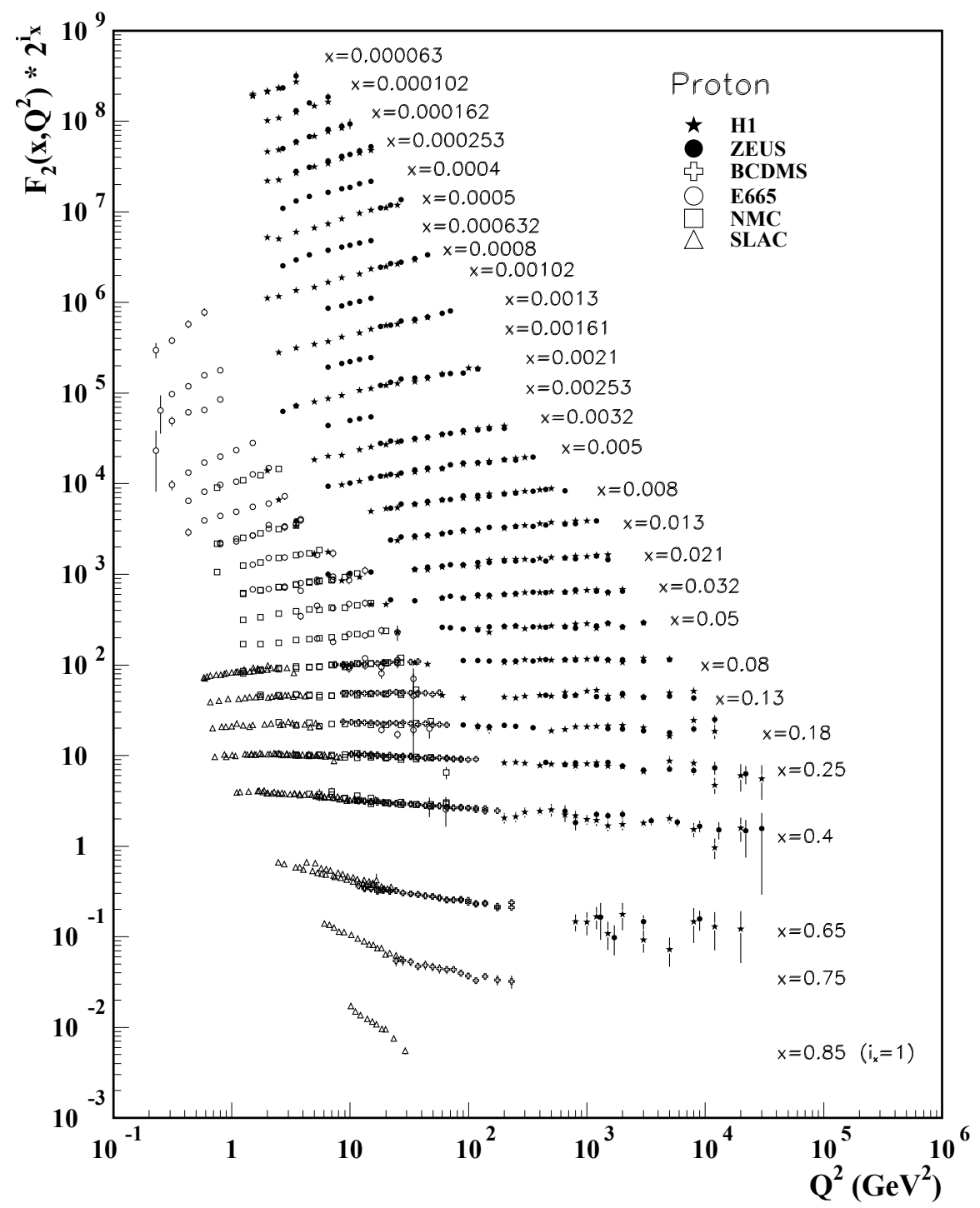

Figure 2.5: The $F_{2}^{p}$ structure function $Q^{2}$-dependence for a range of Bjorken $x$ values [19]. For the purpose of plotting, $F_{2}$ has been multiplied by $2^{i_{x}}$, where $i_{x}$ is the number of $x$ bins, ranging from $i_{x}=1(\mathrm{x}=0.85)$ to $i_{x}=28(\mathrm{x}=0.000063)$. 


$$
L_{\mu \nu}^{A}\left(k, s ; k^{\prime}\right)=2 m \epsilon_{\mu \nu \gamma \delta} s^{\gamma}\left(k-k^{\prime}\right)^{\delta}
$$

where $m$ is the mass of the lepton, $g_{\mu \nu}$ is the metric tensor, and $\epsilon_{\mu \nu \gamma \delta}$ is the Levi-Civita tensor. The hadronic tensor can be divided into similar, but more complicated spin-independent and spin-dependent parts, given by

$$
\begin{gathered}
W_{\mu \nu}^{S}(q ; p)=2\left(-g_{\mu \nu}+\frac{q_{\mu} q_{\nu}}{q^{2}}\right) F_{1}\left(x, Q^{2}\right) \\
+\frac{2}{p \cdot q}\left[\left(p_{\mu}-\frac{p \cdot q}{q^{2}} q_{\mu}\right)\left(p_{\nu}-\frac{p \cdot q}{q^{2}} q_{\nu}\right)\right] F_{2}\left(x, Q^{2}\right) \\
W_{\mu \nu}^{A}(q ; p, S)=2 M \epsilon_{\mu \nu \gamma \delta} q^{\gamma}\left[S^{\delta} g_{1}\left(x, Q^{2}\right)+\left(S^{\delta}-\frac{S \cdot q}{p \cdot q} p^{\delta}\right) \frac{g_{2}\left(x, Q^{2}\right)}{p \cdot q}\right]
\end{gathered}
$$

The dimensionless quantities $F_{1}$ and $F_{2}$ are known as unpolarized structure functions, as they do not depend on the spin of the nucleon, while $g_{1}$ and $g_{2}$ are spindependent quantities and are therefore known as polarized structure functions. Using Equations 2.32 and 2.33 , we can write the differential cross-section as

$$
\left(\frac{d^{2} \sigma}{d \Omega d E^{\prime}}\right)=\frac{\alpha^{2}}{2 M Q^{4}} \frac{E^{\prime}}{E}\left[L_{\mu \nu}^{S} W^{\mu \nu(S)}-L_{\mu \nu}^{A} W^{\mu \nu(A)}\right],
$$

where the terms with opposite symmetry vanish due to the parity conservation of the electromagnetic interaction.

Unpolarized cross-section The spin-independent structure functions $F_{1}$ and $F_{2}$ can be determined from the unpolarized cross-section by

$$
\left(\frac{d^{2} \sigma}{d \Omega d E^{\prime}}\right)=\left(\frac{d \sigma}{d \Omega}\right)_{\mathrm{Mott}}\left[\frac{2}{M} F_{1}\left(x, Q^{2}\right) \tan ^{2} \frac{\theta}{2}+\frac{1}{\omega} F_{2}\left(x, Q^{2}\right)\right]
$$

Figure 2.5 shows a collection of data for the structure functions $F_{2}$ as a function of $Q^{2}$ for different $x$.

Polarized cross-section The spin-dependent structure functions $g_{1}$ and $g_{2}$ can be measured by using two different target spin orientations with respect to the electron beam polarization: longitudinal and transverse. The electron 
has spin $\uparrow$ or $\downarrow$ either parallel or anti-parallel to the beam direction, and the target spin $\Uparrow$ is parallel to the direction of the electron's momentum. The cross-section difference between the two spin states is:

$$
\Delta \sigma_{\|}=\frac{4 \alpha^{2}}{M \nu Q^{2}} \frac{E^{\prime}}{E}\left[\left(E+E^{\prime} \cos \theta\right) g_{1}\left(x, Q^{2}\right)-2 M x g_{2}\left(x, Q^{2}\right)\right]
$$

with

$$
\Delta \sigma_{\|}=\frac{d^{2} \sigma^{\downarrow \uparrow}}{d \Omega d E^{\prime}}-\frac{d^{2} \sigma^{\uparrow \Uparrow}}{d \Omega d E^{\prime}}
$$

For a transversely polarized target, $\Rightarrow$ denotes that the target spin is perpendicular (while in the scattering plane) to the electron beam direction. The polarized cross-section difference is

$$
\Delta \sigma_{\perp}=\frac{4 \alpha^{2}}{M \omega Q^{2}} \frac{E^{\prime 2}}{E}\left[g_{1}\left(x, Q^{2}\right)+\frac{2 E}{\omega} g_{2}\left(x, Q^{2}\right)\right] \sin \theta
$$

with

$$
\Delta \sigma_{\perp}=\frac{d^{2} \sigma^{\downarrow \Rightarrow}}{d \Omega d E^{\prime}}-\frac{d^{2} \sigma^{\uparrow \Rightarrow}}{d \Omega d E^{\prime}}
$$

The combination of measurements on longitudinally and transversely polarized targets can be used to extract the polarized structure functions. 


\section{Chapter 3 \\ Two Photon Physics}

In recent decades, people always study the form factors extracted from elastic scattering experiments to obtain the nucleon and nuclear structure information. Following the well-established formalism discussed in the last chapter, the experimental cross-sections can be interpreted in terms of elastic form factors in Born approximation (one photon exchange approximation). This approach is based on the assumption that the corrections for two-photon processes are relatively small and can be adequately calculated. However, as new precision data has become available, the importance of two-photon processes can not be ignored.

\subsection{The Born Approximation}

The Born Approximation is a method for calculating scattering processes which truncate Figure 2.3 after the very first Feynman diagram. Following [21], for the electron-electron scattering as shown in Figure 2.2, the transition amplitude can be written as:

$$
T_{f i}=-i \int j_{\mu}(x)\left(\frac{1}{q^{2}}\right) j^{\mu}(x) d^{4} x,
$$

where $j^{\mu}$ is the electron current density which can be expressed as

$$
j^{\mu}=-e \bar{\psi} \gamma^{\mu} \psi
$$

When it comes to nucleons, with their internal structure, the current density can no longer be written as Equation (3.2), which is the current density of a 
point-like particle. Instead the hadronic current is

$$
J^{\mu}=-e \bar{\psi} \Gamma^{\mu} \psi
$$

The electromagnetic hadron current operator $\Gamma^{\mu}$ is parameterized by the Dirac $\left(F_{1}\right)$ and Pauli $\left(F_{2}\right)$ form factors as

$$
\Gamma^{\mu}(q)=\gamma^{\mu} F_{1}\left(Q^{2}\right)+\frac{\kappa}{2 M} F_{2}\left(Q^{2}\right) i \sigma^{\mu \nu} q_{\nu}
$$

Here, $\kappa$ is the anomalous magnetic moment, $\sigma^{\mu \nu}=\frac{1}{2}\left[\gamma^{\mu}, \gamma^{\nu}\right]$, and $M$ is the nucleon mass. Then the transition amplitude becomes

$$
\begin{aligned}
T_{f i}= & -i \int j_{\mu}(x)\left(\frac{1}{q^{2}}\right) J^{\mu}(x) d^{4} x \\
= & -i \int\left(-e \bar{\psi} \gamma^{\mu} \psi\right) \times\left(\frac{1}{q^{2}}\right) \\
& \times\left[-e \bar{\psi}\left(\gamma^{\mu} F_{1}\left(Q^{2}\right)+\frac{\kappa}{2 M} F_{2}\left(Q^{2}\right) i \sigma^{\mu \nu} q_{\nu}\right) \psi\right] d^{4} x .
\end{aligned}
$$

The Born approximation assumes all higher-order processes contribute a negligible amount to the cross-section. They are viewed as radiative corrections. Experimentally one only needs the radiative corrections to be precisely calculable. Radiative corrections to cross-sections around $15 \%$ or so are common, and have been done for years. They include the diagrams from (b) to (n) in Figure 2.3. These diagrams were viewed as well enough understood. But the box and cross diagrams were evaluated in the limit that one of the virtual photons was $Q^{2} \approx 0$. Almost all of the cross-section formalism which was presented previously is only valid in the Born Approximation. If one wants to include higher order diagrams, terms such as electron-nucleon scattering invariant amplitude, $T$, must be modified to reflect this. 


\subsection{The Importance of Two-Photon Physics}

In the proton form factors measurement, very different results at large $Q^{2}$ for the ratio $\mu_{p} G_{E}^{p} / G_{M}^{p}$ were observed in two sets of experiments [21] [22] [23]. Two independent methods (Rosenbluth separation [16] and polarization transfer measurement) were used to measure the form factor ratio, the sensitivities to two-photon exchange corrections at large $Q^{2}$ are different. It has been proposed [24] 25] that they may be at least partially reconciled by considering the effects of two-photon exchange on elastic electron-nucleon scattering.

Rosenbluth Method: We re-write Equation 2.12 as:

$$
\left.d \sigma_{R}=C_{B}\left(Q^{2}, \epsilon\right)\left[G_{M}^{2}\left(Q^{2}\right)+\frac{\epsilon}{\tau} G_{E}^{2}\left(Q^{2}\right)\right)\right]
$$

where $C_{B}\left(Q^{2}, \epsilon\right)$ is simply a kinematic factor and $\epsilon$ is the photon polarization parameter, defined as:

$$
\epsilon=\left(1+2(1+\tau) \tan ^{2}\left(\frac{\theta}{2}\right)\right)^{-1}
$$

For a fixed $Q^{2}$, one measures the cross-section for different values of $\epsilon$ to determine the form factors $G_{E}$ and $G_{M}$. Note that in Rosenbluth equation, the cross-sections are sensitive to $G_{E}^{2}$ and $G_{M}^{2}$, so the signs on the $G_{E}$ and $G_{M}$ cannot be determined from Rosenbluth separations alone.

Polarization Method: One can also access the Sachs form factors using a longitudinally polarized electron beam combined with a unpolarized proton target, $\overrightarrow{e p} \rightarrow e \vec{p}$, detect the polarization of the recoiling proton. In the Born approximation, the elastic cross-section for scattering a longitudinally polarized electron from an unpolarized proton, producing a longitudinally polarized recoil proton is given by [26]:

$$
\left(\frac{d \sigma}{d \Omega}\right)^{l}=h\left(\frac{d \sigma}{d \Omega}\right)_{\mathrm{Mott}} \frac{E+E^{\prime}}{M} \sqrt{\frac{\tau}{1+\tau}} \tan ^{2}\left(\frac{\theta}{2}\right) G_{M}^{2},
$$


where $h$ is the electron helicity. And the cross-section for producing a transversely polarized recoil proton is given by [26]:

$$
\left(\frac{d \sigma}{d \Omega}\right)^{t}=-h\left(\frac{d \sigma}{d \Omega}\right)_{\mathrm{Mott}} 2 \sqrt{\frac{\tau}{1+\tau}} \tan \left(\frac{\theta}{2}\right) G_{E} G_{M}
$$

In the Born approximation the normal polarization is identically 0 [26]. The ratio of the proton electric form factor to proton magnetic form factor can be obtained by taking the ratio of the transverse to longitudinal proton cross-sections:

$$
\frac{P_{t}}{P_{l}}=-\frac{2 M \cot \left(\frac{\theta}{2}\right)}{E+E^{\prime}} \frac{G_{E}}{G_{M}}=-\sqrt{\frac{2 \epsilon}{\tau(1+\epsilon)}} \frac{G_{E}}{G_{M}},
$$

where $P_{l}\left(P_{t}\right)$ is the polarization of the recoil proton longitudinal (transverse) to the proton momentum in the scattering plane. $P_{l}$ is proportional to the longitudinal cross-section in Equation 3.10 and $P_{t}$ is proportional to the transverse cross-section in Equation (3.11).

As shown in Figure 3.1, very different results were observed in these two sets of experiments. It was pointed out that the discrepancy in $G_{E} / G_{M}$ can be explained as a possible failure of the Born approximation when two-photonexchange contributions are considered [24][25][29]. Under Lorentz, parity and charge conjugation invariance, if one neglects the mass of the electron, the Tmatrix becomes [25]:

$$
T=\frac{e^{2}}{Q^{2}} \bar{\psi}\left(k^{\prime}\right) \gamma_{\mu} \psi(k) \times \bar{\psi}\left(p^{\prime}\right)\left(\tilde{G}_{M} \gamma^{\mu}-\tilde{F}_{2} \frac{P^{\mu}}{M}+\tilde{F}_{3} \frac{\gamma \cdot K P^{\mu}}{M^{2}}\right) \psi(p),
$$

where $K=\left(k+k^{\prime}\right) / 2$ and $P=\left(p+p^{\prime}\right) / 2$. The quantities $\tilde{G}_{M}, \tilde{F}_{2}$ and $\tilde{F}_{3}$ contain information about nucleon structure. When returning to the Born approximation, we recover the usual electric and magnetic nucleon form factors. Thus, the generalized form factors can be written in terms of the Born form factors as:

$$
\tilde{G}_{M}\left(\omega, Q^{2}\right)=G_{M}^{B o r n}\left(Q^{2}\right)+\delta \tilde{G}_{M}
$$




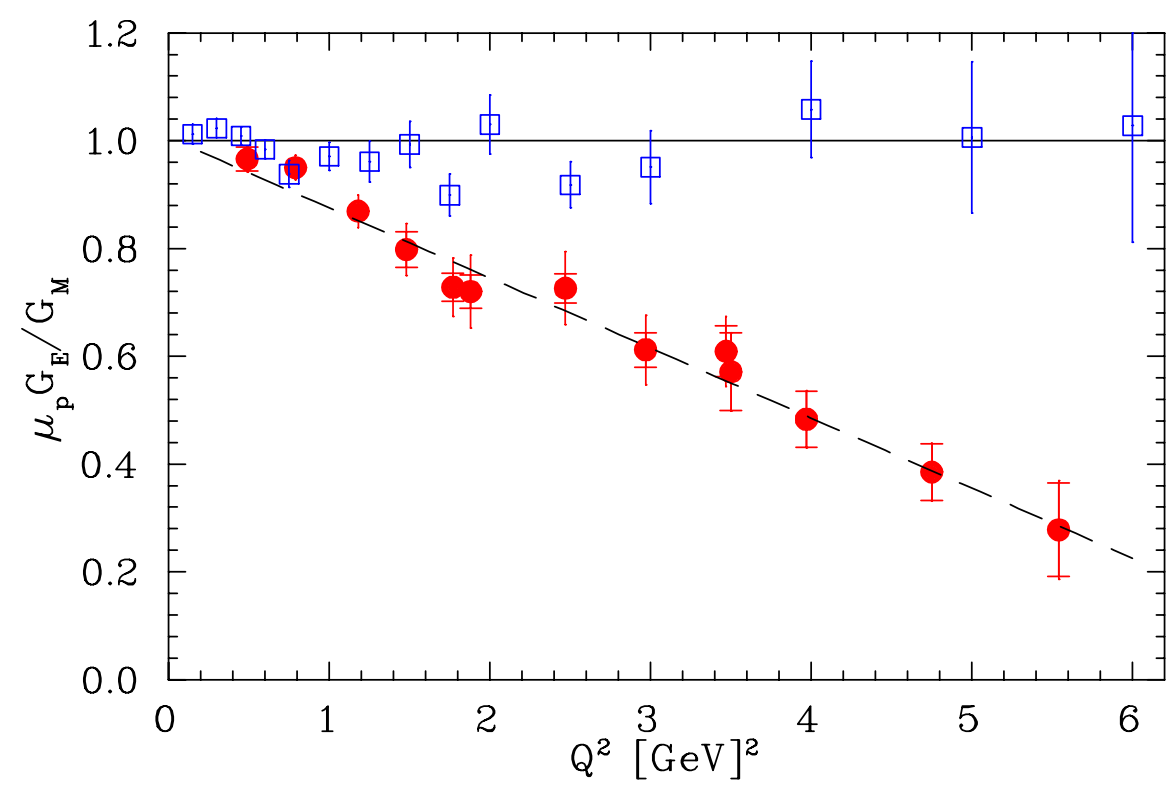

Figure 3.1: Ratio of proton electric form factor to magnetic form factor. Data from the Rosenbluth separations are shown in open squares and data from the polarization transfer measurements are shown in filled circles. The dash line is a fit of the polarization transfer data. Figure is from [27] of data from [22], [23] and [28].

$$
\begin{gathered}
\tilde{F}_{2}\left(\omega, Q^{2}\right)=F_{2}^{B o r n}\left(Q^{2}\right)+\delta \tilde{F}_{2} \\
\tilde{F}_{3}\left(\omega, Q^{2}\right)=0+\delta \tilde{F}_{3},
\end{gathered}
$$

where the $\delta$ terms indicate the contribution from multi-photon exchange.

The Rosenbluth and polarization transfer expressions can now be written in terms of these multi-photon form factors [25]:

$$
\frac{\sigma_{R}}{C_{B}\left(Q^{2}, \epsilon\right)} \simeq \frac{\left|\tilde{G}_{M}\right|^{2}}{\tau}\left\{\tau+\epsilon \frac{\left|\tilde{G}_{E}\right|^{2}}{\left|\tilde{G}_{M}\right|^{2}}+2 \epsilon\left(\tau+\frac{\left|\tilde{G}_{E}\right|}{\left|\tilde{G}_{M}\right|}\right) \mathcal{R}\left(\frac{\omega \tilde{F}_{3}}{M^{2}\left|\tilde{G}_{M}\right|}\right)\right\}
$$

and

$$
\frac{P_{t}}{P_{l}} \simeq-\sqrt{\frac{2 \epsilon}{\tau(1+\epsilon)}}\left\{\frac{\left|\tilde{G}_{E}\right|}{\left|\tilde{G}_{M}\right|}+\left(1-\frac{2 \epsilon}{1+\epsilon} \frac{\left|\tilde{G}_{E}\right|}{\left|\tilde{G}_{M}\right|}\right) \mathcal{R}\left(\frac{\omega \tilde{F}_{3}}{M^{2}\left|\tilde{G}_{M}\right|}\right)\right\},
$$

where $\mathcal{R}$ denotes the real part, and the notation $\tilde{G}_{E}=\tilde{G}_{M}-(1+\tau) \tilde{F}_{2}=$ $G_{E}\left(\nu, Q^{2}\right)+\delta \tilde{G}_{E}$ has been used. Application of the correction appears to bring the two methods into better agreement, as shown in Figure 3.2 and Figure 3.3 . The corrected results are clearly in better agreement with the data. 


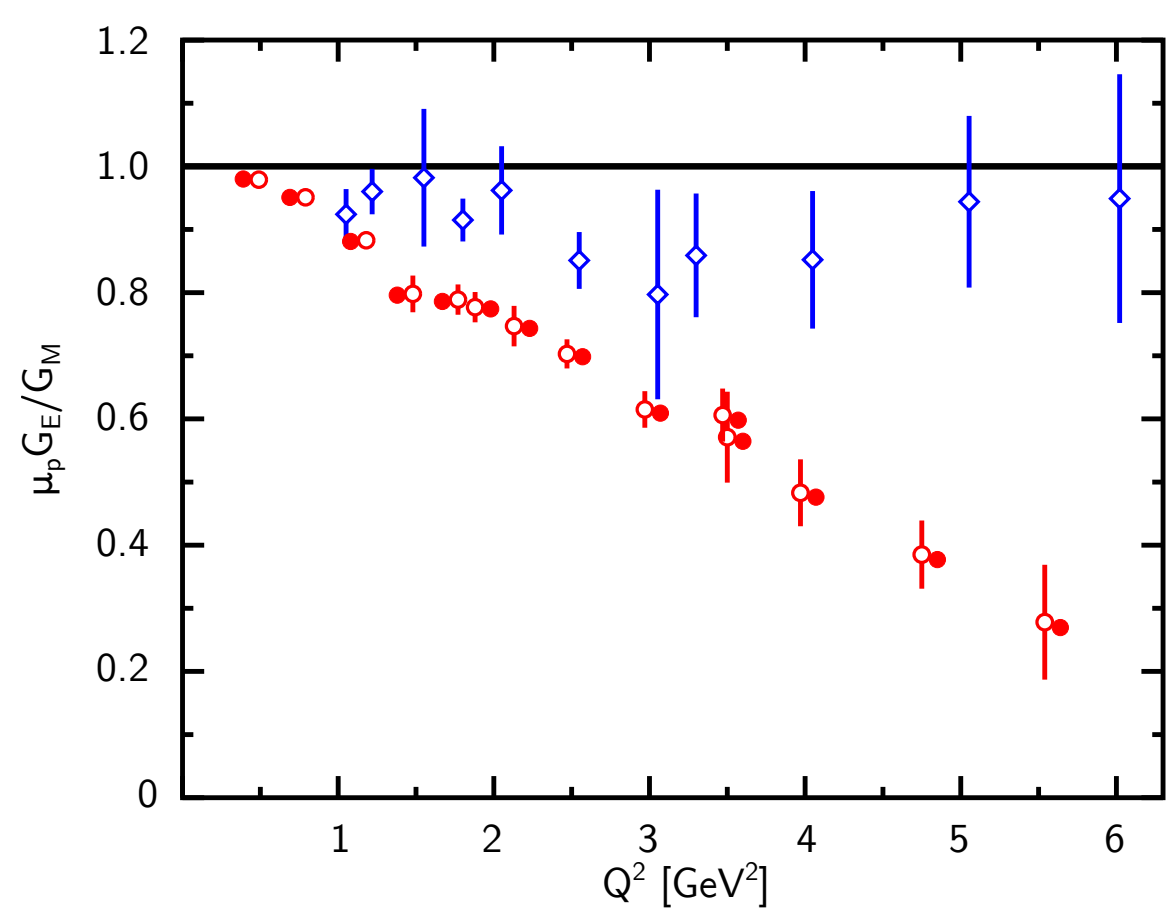

Figure 3.2: Proton $\mu G_{E} / G_{M}$ ratio obtained from the polarization transfer measurements [22, with (filled circles) and without (open circles) two-photonexchange corrections [30]. The values have been offset in $x$ axis for clarity. The Rosenbluth separations ratio [27] (open diamonds) is shown for comparison.

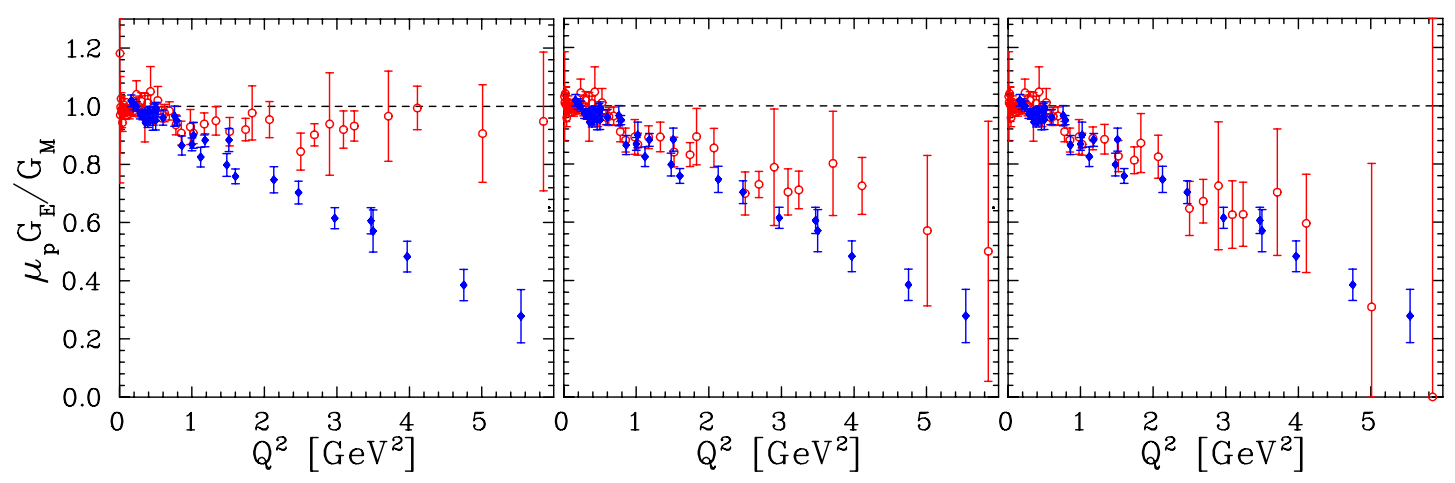

Figure 3.3: Comparison of polarization transfer measurements (filled diamonds) and Rosenbluth separations (open circles) without two-photon-exchange corrections (left), with two-photon-exchange corrections from [30] (center), and with the additional high- $Q^{2}$ correction applied in [31] (right). Figure is reproduced from [26]. 


\subsection{Experimental Observable for Two Photon Exchange}

Polarized target: An observable which is related to two-photon exchange is the asymmetry for elastic scattering of an unpolarized electron on a nucleon polarized normal to the scattering plane:

$$
A_{y}=\frac{\sigma^{\uparrow}-\sigma^{\downarrow}}{\sigma^{\uparrow}+\sigma^{\downarrow}}
$$

where $\sigma^{\uparrow}\left(\sigma^{\downarrow}\right)$ is the cross-section for an unpolarized beam and for target spin parallel (anti-parallel) to the normal polarization vector as defined in Section 3.4. As [32] showed, the target normal single spin asymmetry is related to the imaginary part introduced by two photon exchange amplitude as

$$
A_{y}=\frac{2 \mathcal{I}\left(T_{1 \gamma}^{*} T_{2 \gamma}\right)}{\left|T_{1 \gamma}\right|^{2}}
$$

where $\mathcal{I}$ denotes the imaginary part and $T_{1 \gamma}$ denotes the one-photon exchange amplitude.

Polarized electron: The two-photon-exchange can also be accessed by measuring the electron beam asymmetry for electrons polarized normal to the scattering plane scattering from unpolarized targets [26]. Time reversal invariance requires that $B_{y}=A_{y}[32$. The corresponding beam normal asymmetry $B_{y}$ is then defined analogously to Equation 3.19, with the electron spin parallel or anti-parallel to the normal polarization vector.

\subsection{Define Up and Down}

In order to compare theoretical predictions and experimental results, it is necessary that a clear definition of "up" and "down" be given as it applies to Equation (3.19). The scattering plane is defined by the incoming and outgoing electron 
momentum, $\vec{k}$ and $\overrightarrow{k^{\prime}}$. Then $\hat{n}$, which is normal to the scattering plane, is defined as

$$
\hat{n}=\frac{\vec{k} \times \overrightarrow{k^{\prime}}}{\left|\vec{k} \times \overrightarrow{k^{\prime}}\right|}
$$

If the target spin is parallel to $\hat{n}$, we define it as spin up $(\uparrow)$, while the target spin is anti-parallel to $\hat{n}$ is defined as spin down $(\downarrow)$.

\subsection{Existing Data}

Proton data: Using an unpolarized electron beam and a polarized proton target, within the large experimental uncertainties, $A_{y}$ was observed to be consistent with zero up to $Q^{2}=0.98 \mathrm{GeV}^{2}$ [33], as shown in Figure 3.4 (a). There are also several measurements have been made in elastic ep scattering using a polarized electron beam and an unpolarized proton target [34] [35] [36] [37] [38] [39] [40], the results are shown in Figure 3.4 (b) and (c).

Neutron data: An attempt of measuring $A_{y}$ in the ${ }^{3} \mathrm{He}\left(e, e^{\prime}\right)$ reaction at $Q^{2}=0.1$ $\mathrm{GeV}^{2}$ was made at NIKHEF [41]. Here, $A_{y}$ for quasi-elastic scattering was found to be $A_{y}=-0.095 \pm 0.054$, which is not significantly different from zero. Also, experiments have been performed at Jefferson Lab to measured the target normal asymmetry of the neutron on a ${ }^{3}$ He target for deep-inelastic scattering [42] and quasi-elastic scattering. This dissertation mainly focuses on the later reaction.

\subsection{Jefferson Lab E05-015}

This dissertation reports the most recent measurement of target single-spin asymmetries, $A_{y}^{3} \mathrm{He}$, through electron scattering from a normally polarized Helium-3 target in the quasi-elastic process ${ }^{3} \mathrm{He}^{\uparrow}\left(e, e^{\prime}\right)$ at $Q^{2}$ values of $0.13,0.46$ and 0.97 $\mathrm{GeV}^{2}$. It also reports the neutron single-spin asymmetries, $A_{y}^{n}$, extracted from 


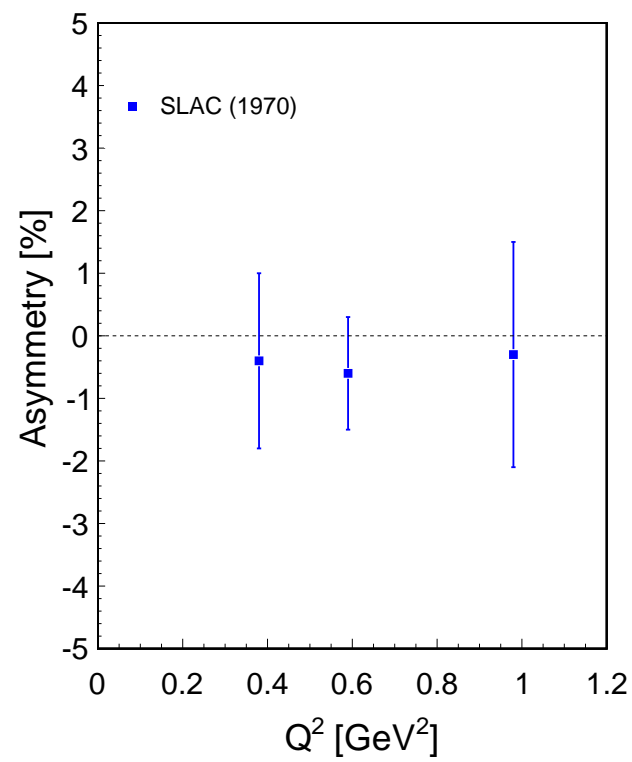

(a)

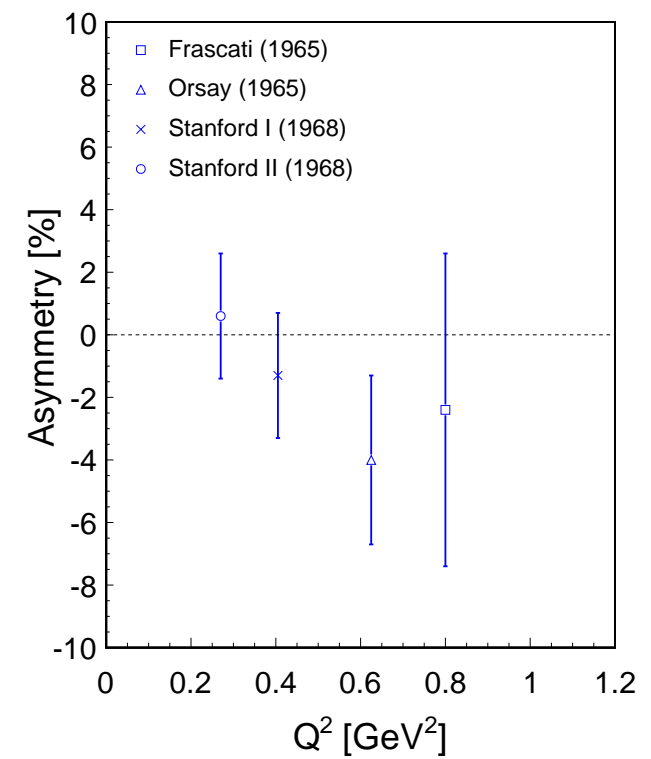

(b)

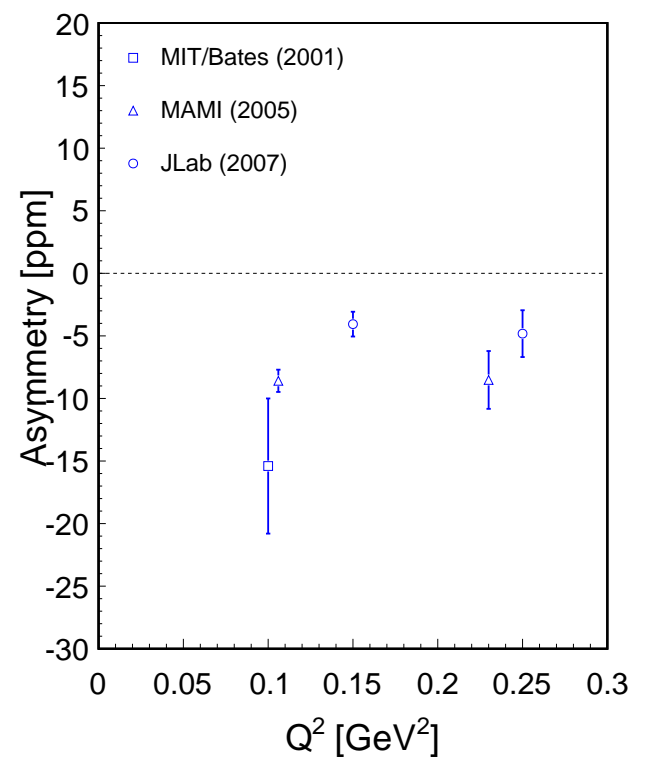

(c)

Figure 3.4: Previous asymmetry measurements from elastic electron-proton scattering. (a) SLAC measured the asymmetry for scattering electrons from a polarized proton target. (b) and (c) Measured electron beam asymmetry for electrons polarized normal to the scattering plane scattering from unpolarized proton target. The error bars are statistical uncertainties. 


\begin{tabular}{|c|cc|c|}
\hline Group & $Q^{2}$ & $A_{y}^{p}$ & Year \\
\hline Frascati[34] & 0.80 & $-0.024 \pm 0.050$ & 1965 \\
\hline Orsay[35] & 0.625 & $-0.040 \pm 0.027$ & 1965 \\
\hline Stanford I[36] & 0.405 & $-0.013 \pm 0.020$ & 1968 \\
\hline Stanford II[37] & 0.27 & $0.006 \pm 0.020$ & 1968 \\
\hline & 0.38 & $-0.004 \pm 0.014$ & \\
SLAC[33] & 0.59 & $-0.006 \pm 0.009$ & 1970 \\
& 0.98 & $-0.003 \pm 0.018$ & \\
\hline MIT/Bates[38] & 0.1 & $-15.4 \pm 5.4 \mathrm{ppm}$ & 2001 \\
\hline MAMI[39] & 0.106 & $-8.59 \pm 0.89 \pm 0.75 \mathrm{ppm}$ & \multirow{2}{*}{2005} \\
& 0.230 & $-8.52 \pm 2.31 \pm 0.87 \mathrm{ppm}$ & \\
\hline \multirow{2}{*}{ JLab[40] } & 0.15 & $-4.06 \pm 0.99 \pm 0.63 \mathrm{ppm}$ & \multirow{2}{*}{2007} \\
\hline
\end{tabular}

Table 3.1: Summary of the $A_{y}^{p}$ measurements in the past years. SLAC measured the asymmetry for scattering electrons from a polarized proton target (unpolarized electron with a polarized target). The other values were from analyzing the polarization of the protons recoiling from an unpolarized proton target (polarized electron with an unpolarized target).

the measured ${ }^{3} \mathrm{He}$ asymmetries. The measurement was performed in Hall A at Jefferson Laboratory in Newport News, VA. The rest of this dissertation will give a detailed report on this experiment, as well as the analysis of the data and its results. 


\section{Chapter 4 Experimental Setup}

E05-015 was conducted in Experimental Hall A of the Thomas Jefferson National Accelerator Facility from April 24 to May 12, 2009. The purpose of the experiment was to make a precision measurement of the target single-spin asymmetry,

$A_{y}^{3} \mathrm{He}\left(A_{y}^{n}\right)$, through electron scattering from a vertically polarized ${ }^{3} \mathrm{He}$ target in the quasi-elastic region. JLab's continuous electron beam (Section 4.2) and the Hall A polarized ${ }^{3} \mathrm{He}$ target (Chapter 5) make this measurement possible. The target was polarized normal to the scattering plane, and the scattered electrons were simultaneously detected by the two standard Hall A High Resolution Spectrometers (HRSs) (Section 4.5).

\subsection{Jefferson Lab}

Thomas Jefferson National Accelerator Facility, also known as Jefferson Lab (JLab), is the site of the Continuous Electron Beam Accelerator Facility (CEBAF). Jefferson Lab is one of 17 national laboratories funded by the U.S. Department of Energy (DOE). It is located in Newport News, Virginia. The accelerator is able to provide a high quality electron beam up to $6 \mathrm{GeV}$, and is being upgraded to deliver $12 \mathrm{GeV}$. Since the first beam on target in July, 1994, a wide spectrum of physics experiments have been carried out at this facility [43]. The accelerator together with the three experimental Halls have made CEBAF one of the world's leading medium energy nuclear facilities. An aerial view of the CEBAF site, including the three experimental Halls is shown in Figure 4.1. 


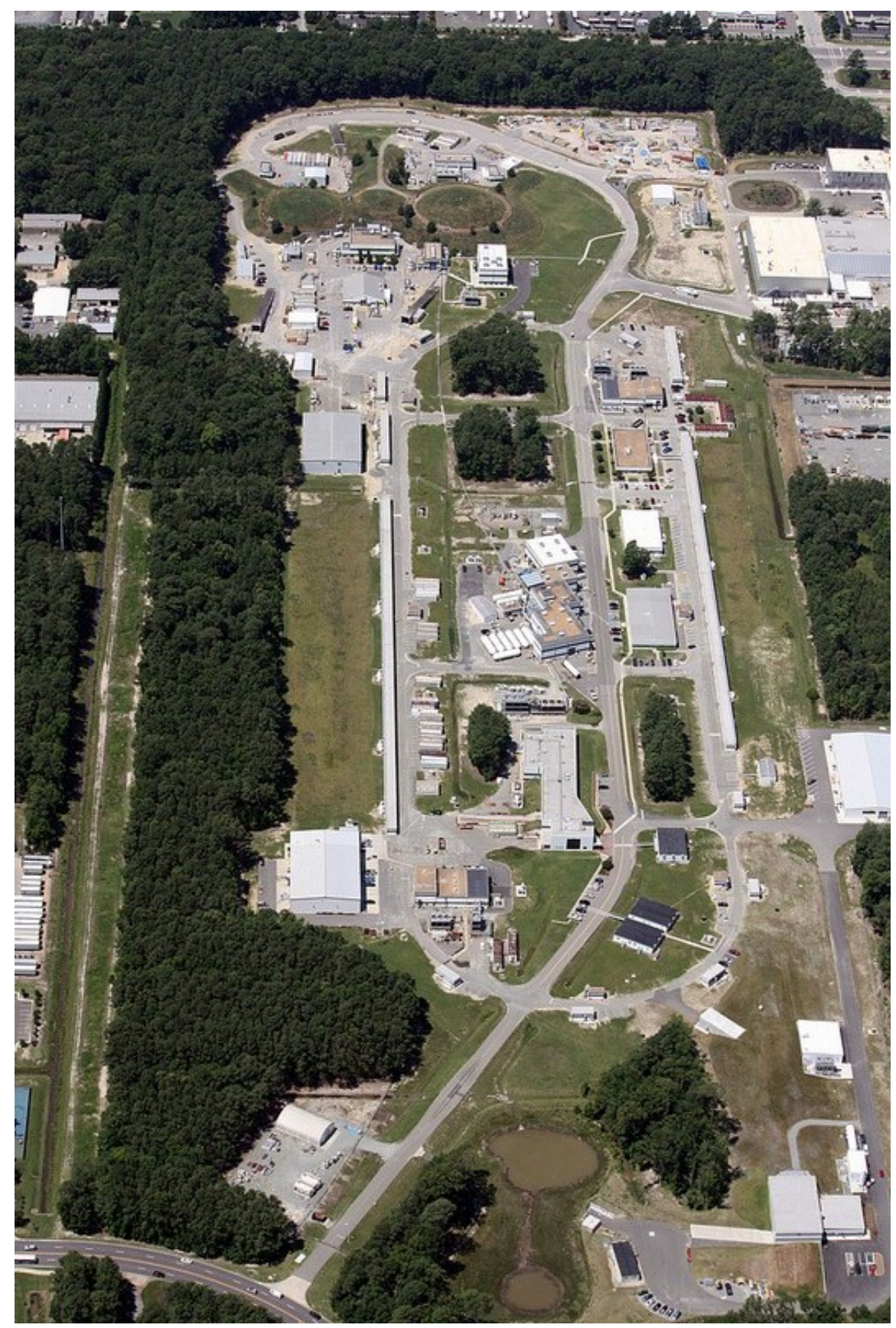

Figure 4.1: An aerial view looking southwest at the Jefferson Lab CEBAF accelerator site and three experimental Halls. 


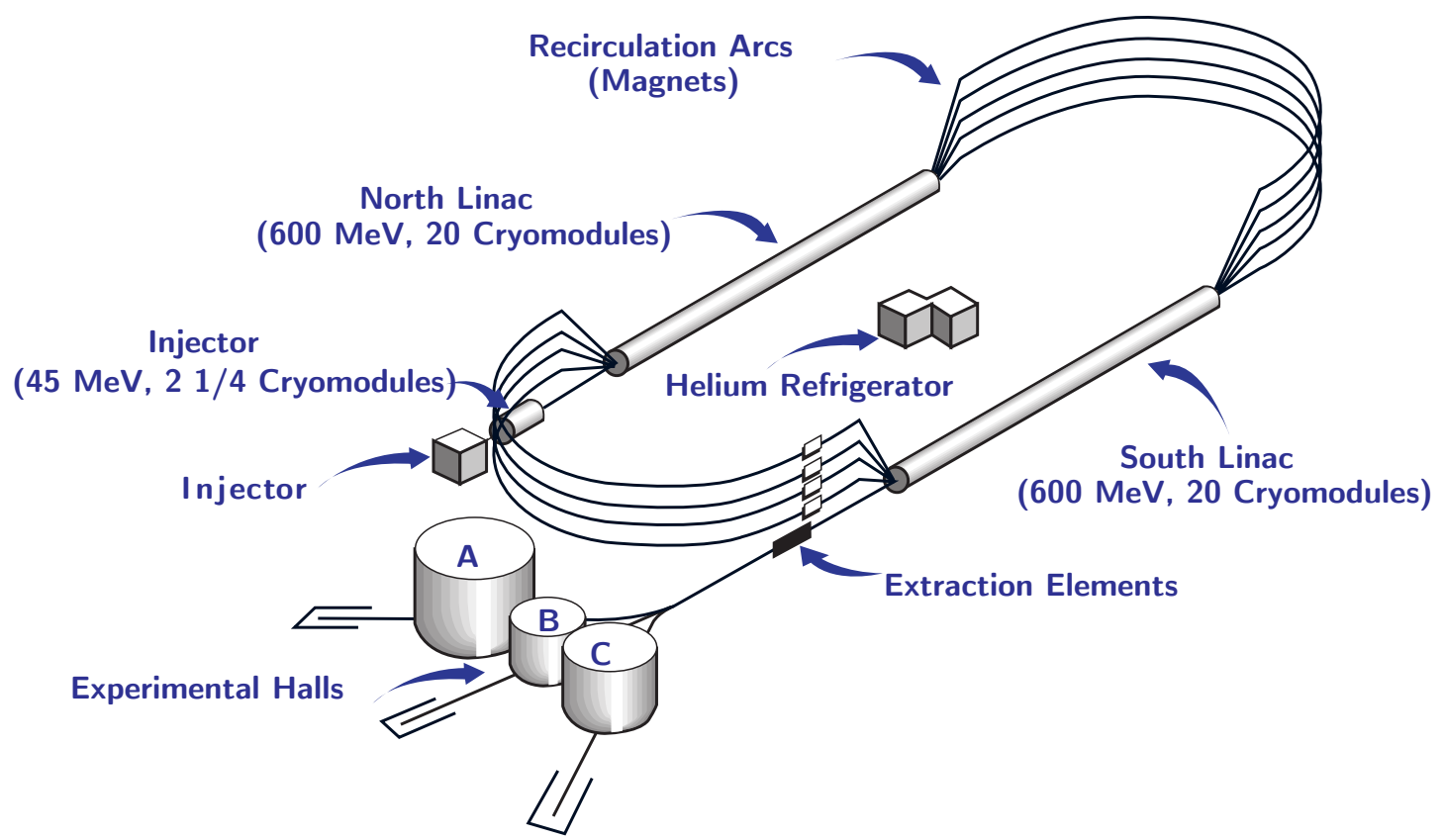

Figure 4.2: Sketch of CEBAF [4]. Polarized electrons are produced in the injector and are accelerated in the two linacs. Two sets of recirculating arcs allow the beam to go through the linacs up to five times. Once the electrons are accelerated, they can be extracted into any of the three experimental Halls: A, B, or C.

\subsection{The Continuous Electron Beam Accelerator Facility}

CEBAF, which generally provides a polarized electron beam, comprises a polarized light source, an injector, two linacs, two re-circulation arcs and a beam switching station to transmit the electron beam into the three experimental Halls: A, B and C. The beam starts at the polarized electron source. It goes through the injector and enters the linacs for acceleration up to $6 \mathrm{GeV}$. Two sets of recirculating arcs are used for recirculating the beam. The beam can be circulated up to five times with each pass increasing the energy by up to $1.2 \mathrm{GeV}$. The beam can be simultaneously sent to the three experimental Halls by a beam switchyard. The different components of CEBAF are described in detail below. 


\subsubsection{Injector}

The polarized electron beam source is a photocathode, which emits electrons when irradiated by a circularly polarized, pulsed laser at a certain energy [44]. The bunch repetition rate of the beam is determined by the laser's pulse rate and the helicity of the electrons is determined by the polarization of the laser. The photocathode is made of strained gallium arsenide (GaAs); it consists of several layers of GaAs. When the photocathode absorbs photons of an appropriate energy, the electrons are excited to the conduction band; in the presence of a large external electric field, the electrons then diffuse to the surface and escape from the photocathode 45].

The laser polarization was changed every $33.3 \mathrm{~ms}$, which caused a flip of the electrons' helicity every $33.3 \mathrm{~ms}$ [46]. Since E05-015 measured the target singlespin asymmetry, the electrons do not necessarily to be polarized, the helicity of the beam was integrated over during the analysis of the data. On the photocathode, there is a constant $-100 \mathrm{kV}$ voltage. The constant electric field generated by this voltage helps extract electrons and gives an energy of $100 \mathrm{keV}$ to the electrons on their way to the injector. At the injector, electrons are accelerated from their initial energy of $100 \mathrm{keV}$ to a final energy of $45 \mathrm{MeV}$ by passing through two quarter-cryomodules. Each quarter-crymodule consists of two superconducting radio-frequency (SRF) cavities. These two cryomodules are referred to as a quarter-cryomodule, since each of the cryomodules in the main accelerator consists of eight SRF cavities [47].

\subsubsection{Linear Accelerator}

The north and south SRF linear accelerators (linacs) of CEBAF are shown in Figure 4.2. From the injector, electrons with an initial energy of $45 \mathrm{MeV}$ enter the north linear accelerator section, which consists of 20 cryomodules or 160 SRF 
cavities. The initial accelerator was designed to accelerate the electrons up to 400 $\mathrm{MeV}$; however, due to the high performance of the cavities in the linacs, electrons from the injector can now be accelerated up to $600 \mathrm{MeV}$ before entering the recirculating arc. Electrons are bent $180^{\circ}$ by the recirculation arc after the north linac and gain another $600 \mathrm{MeV}$ from south linac. Due to the unique Jefferson Lab construction, the electrons can be accelerated up to five times through same cavities [48], which means the maximum electron energy can be up to $6 \mathrm{GeV}$.

\subsubsection{Recirculating Arcs}

The recirculating arcs, which bend the electron beam by $180^{\circ}$, consist of a series of dipole magnets. Electrons are separated by a magnet depending on the electron energy and guided into five different beam pipes that go through the arc. The lower energy electrons, which are easier to steer, are guided into the higher arcs, while higher energy electrons are guided into the lower arcs. As a result, each of the lines in the arc has electrons with only one specific energy. The electrons from the five beam pipes are re-combined at the end of the arc to be put into the next linac [48].

\subsubsection{Beam Switchyard}

The beam switchyard is used to extract and simultaneously send beam to up to all three experimental Halls. The beam energy can be taken from any of the five passes through the accelerator [48].

\subsection{Experimental Hall A}

Hall A [49] is the largest of Jefferson Lab's experimental Halls. It is 174 feet across and 80 feet tall from the floor to the highest point on its domed ceiling. The foundation for the hall is 35 feet below ground. The layout of experiment 


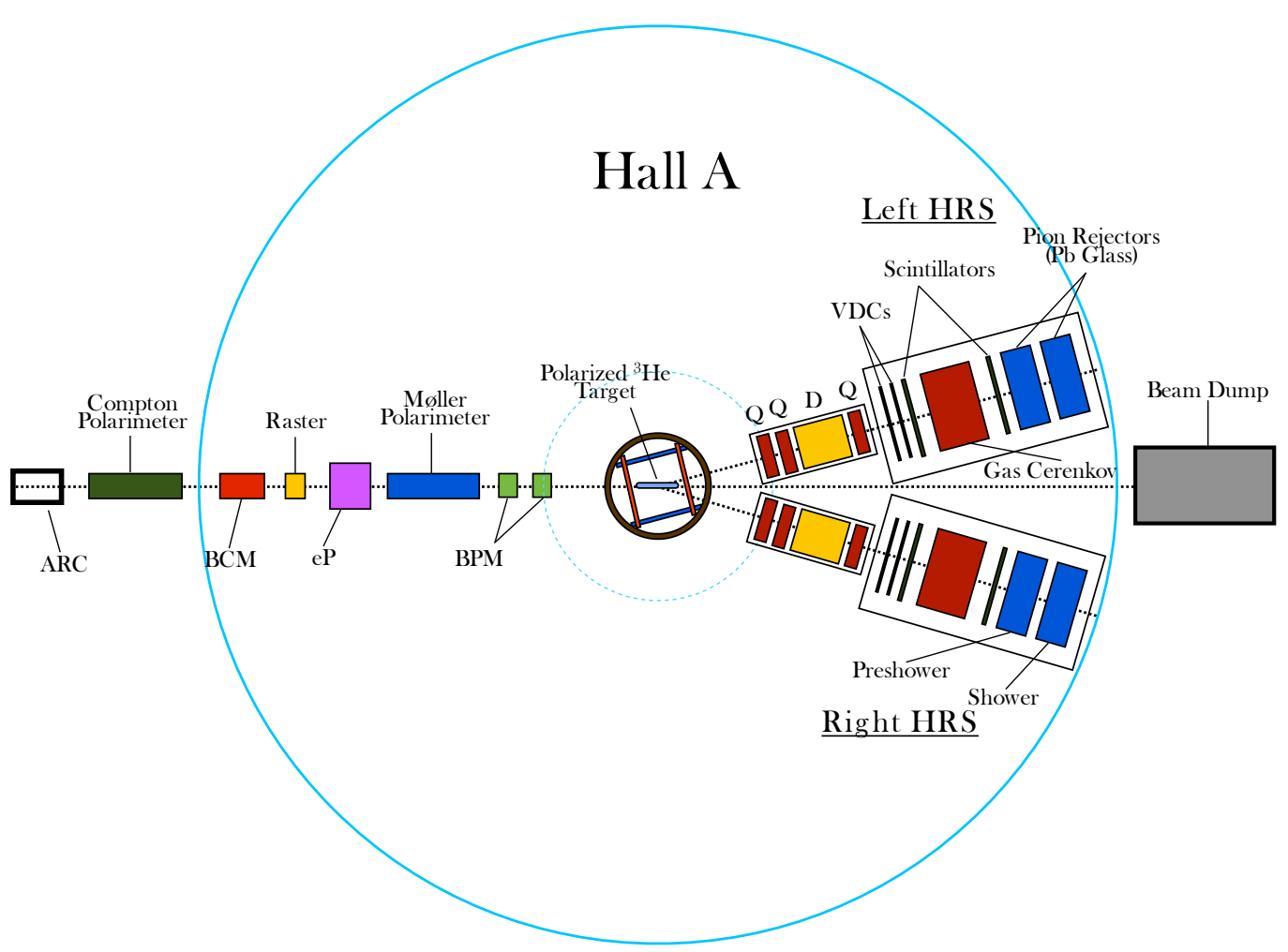

Figure 4.3: Experimental Hall A layout during the E05-015. The electron beam comes into the Hall from the left, passes beam quality monitors, and impinges on the target at the center of the Hall. Scattered electrons were detected by the two High Resolution Spectrometers (HRSs). Electrons that do not interact with the target are stopped in the beam dump.

E05-015 is shown in Figure 4.3. After entering the Hall, before interacting with the target, the electron beam passes through various instruments. These instruments determine different properties of the beam such as beam current, beam energy, and beam position. The electron beam interacts with the target, and the reaction products are detected by two high resolution spectrometers, known as Left-HRS (LHRS) and Right-HRS (RHRS) as they are located to beam left and right, respectively. Electrons that do not interact with the target are stopped in the beam dump. The polarized ${ }^{3}$ He target used in the experiment E05-015 was located at the center of the Hall, known as the pivot. 


\subsection{Beam Line}

The Hall A beam line starts at the beam switchyard and ends at the Hall A beam dump [49]. The beam line consists of the following components:

- arc magnets, which bend the beam into the Hall and provide for a beam energy measurement;

- a Compton polarimeter for the beam polarization measurement;

- two beam current monitors (BCMs) between which an Unser monitor provides an absolute beam current measurement;

- a fast raster;

- the eP device for beam energy measurement;

- a Moller beam polarimeter for beam polarization measurement;

- and a number of beam position monitors (BPMs).

With the use of these devices, the properties of the beam can be precisely determined. The Compton polarimeter, eP device and Moller were not used for the experiment E05-015.

\subsubsection{Beam Energy Measurement}

The beam energy is measured by the Arc measurement [49]. The principle of the Arc measurement is that an electron in a uniform magnetic field moves in a circle with a radius $r$ :

$$
r=\frac{p}{e B}
$$

where $B$ is the strength of the magnetic field, $p$ the electron's momentum and $e$ is the electron charge. In a uniform magnetic magnetic field, the momentum of 


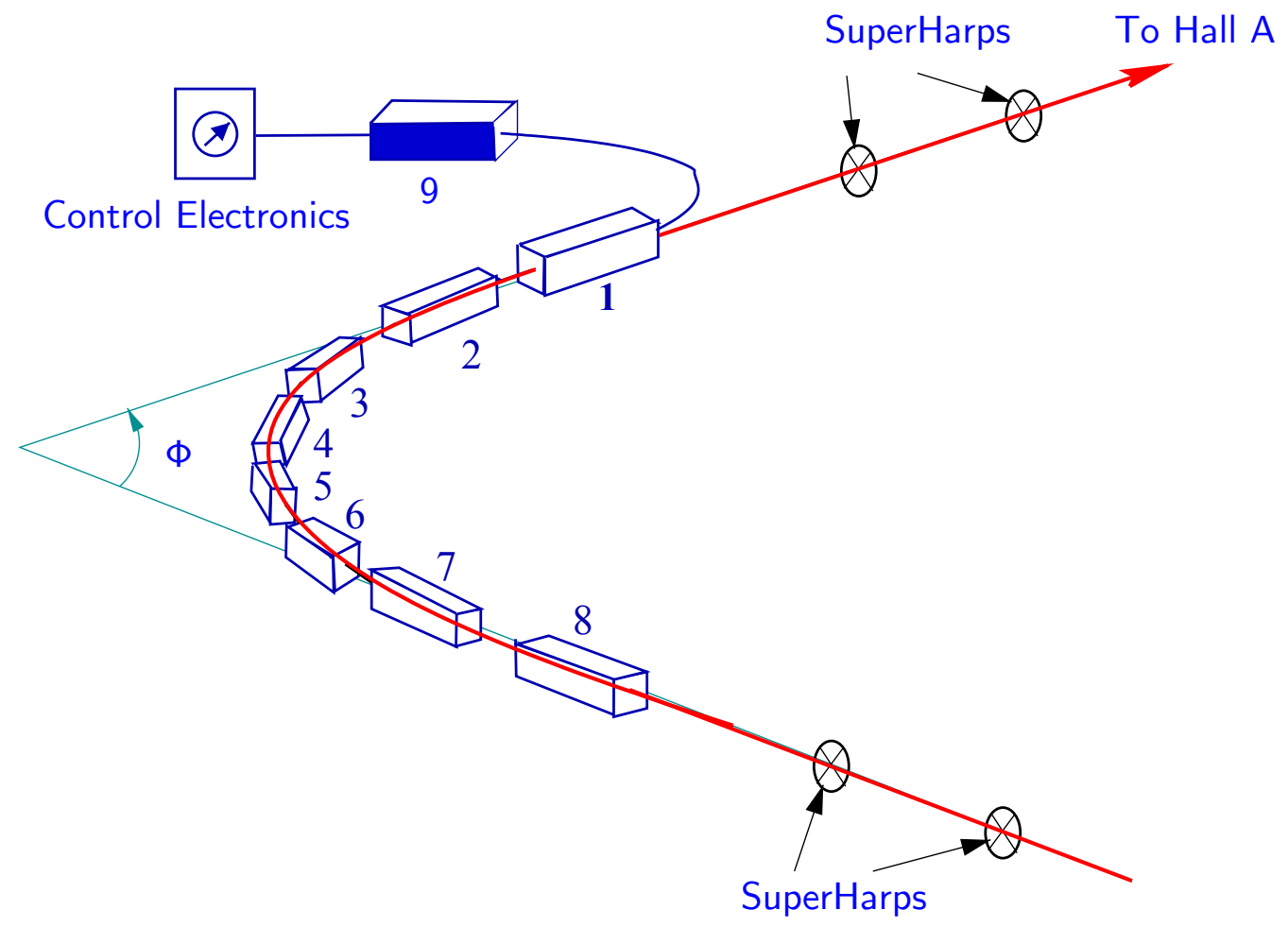

Figure 4.4: The arc section of the beam line [15]. Eight magnets are used to deflect the beam by $34.3^{\circ}$. The position of the beam before and after the arc is determined by the SuperHarps. The magnetic field integral of the eight bending magnets is based on the direct field measurement of the $9^{\text {th }}$ reference magnet.

the electron can be determined by:

$$
p=e B \cdot r=e B \cdot \frac{l}{\phi}=\frac{e}{\phi} \int \vec{B} \cdot d \vec{l}
$$

where $\phi$ is the bending angle of the electron. Accordingly, the momentum of the electron can be determined by measuring its bending angle $\phi$ and trajectory length $l$ after passing through a well-known magnetic field.

As shown in Figure 4.4 , there are in total eight dipole magnets providing the magnetic field in the arc. The magnets are powered in series to ensure the same current through all of them. As the vacuum pipe runs through the arc dipole magnets, which makes access difficult, the magnetic field is instead measured in the $9^{\text {th }}$ dipole magnet, which is also connected in series with the others but outside the beam enclosure. The angle $\phi$ is $34.3^{\circ}$, and the bending angle of the beam 
Table 4.1: Beam energy from Tiefenback measurements for different settings of E05-015.

\begin{tabular}{|c|c|}
\hline Pass & Beam Energy (GeV) \\
\hline 1 & 1.245 \\
2 & 2.425 \\
3 & 3.605 \\
\hline
\end{tabular}

is $\theta=\pi-\phi$. Deviations from $\phi$ can be determined by the wire scanners, also referred to as the "SuperHarps". The detailed information of the wire scan can be found in [50]. The Arc method provides an accuracy of $\mathrm{d} E_{\text {beam }} / E_{\text {beam }} \approx 2 \times 10^{-4}$.

However, the Arc method is an invasive technique, since during the measurement process the SuperHarp wires pass through the beam. Thus it can not be used to monitor the beam energy continuously during the production running. For this, a non-invasive method, known as Tiefenback, for continuously monitoring the beam energy is used. This method uses the Hall A arc beam position monitors in combination with the current values of the arc magnetic field integral to determine the beam energy. The average value for each energy setting is summarized in Table 4.1. The accuracy of this approach is $\mathrm{d} E_{\text {beam }} / E_{\text {beam }} \approx 5 \times 10^{-4}$.

\subsubsection{Beam Current Measurement}

The complete beam current monitoring system is located $25 \mathrm{~m}$ upstream of the target, as illustrated in Figure 4.3. It consists of two RF cavities and an Unser Monitor between the cavities as shown in Figure 4.5. It provides a stable, lownoise and non-intercepting measurement of the beam current [49].

The Unser monitor [51] is a Parametric Current Transformer, which provides an absolute beam current measurement. It is used to calibrate the two RF cavities. It can not be used for continuous monitoring of the electron beam current, because 
its output signal drifts significantly with time. The two RF cavity monitors placed on either side of the Unser monitor, are called the upstream BCM and the downstream BCM. They are typically used to provide continuous monitoring of the electron beam current. The BCMs are stainless steel cylindrical waveguides adjusted to the beam RF frequency of $1.497 \mathrm{GHz}$. The waveguide produces signals proportional to the beam current as the electrons pass through the cavity. The output from each cavity is split into two signals. The first signal goes to a digital multimeter (DMM), which sends out the root mean squre (RMS) value of the input signal once per second to form the sampled signal. The second signal is an integrated signal transformed by an RMS-to-DC converter into an analog DC signal, which is further converted to a frequency by a Voltage-to-Frequency (V-toF) converter. The output is then counted by the VME scalers, thereby providing a measure of the total accumulated charge.

The output of the RMS-to-DC converter is linear for beam currents between 5 and $200 \mu \mathrm{A}$, but the linearity can be extended down to low beam currents by the use of additional amplifiers. For this, two additional amplifiers are used in front of the RMS-to-DC module with gains of $\times 3$ and $\times 10$. As a result, there are in total 6 BCM outputs (u1, u3, u10, d1, d3 and d10), which are all fed into scaler modules of each spectrometer (LHRS and RHRS) and recorded in the data-stream.

\subsubsection{Beam Position Measurement}

There are two beam position monitors (BPMs) located $7.534 \mathrm{~m}$ and $1.286 \mathrm{~m}$ upstream of the target [49]. The BPMs consist of four wire antennas coaxial with the beam. When the electron beam passes through the inside of the antennae array, a signal is induced that is inversely proportional to the distance between the beam and antenna. The BPMs can determine the relative position of the beam to within $100 \mu \mathrm{m}$ for currents above $1 \mu \mathrm{A}$. They are calibrated by two 


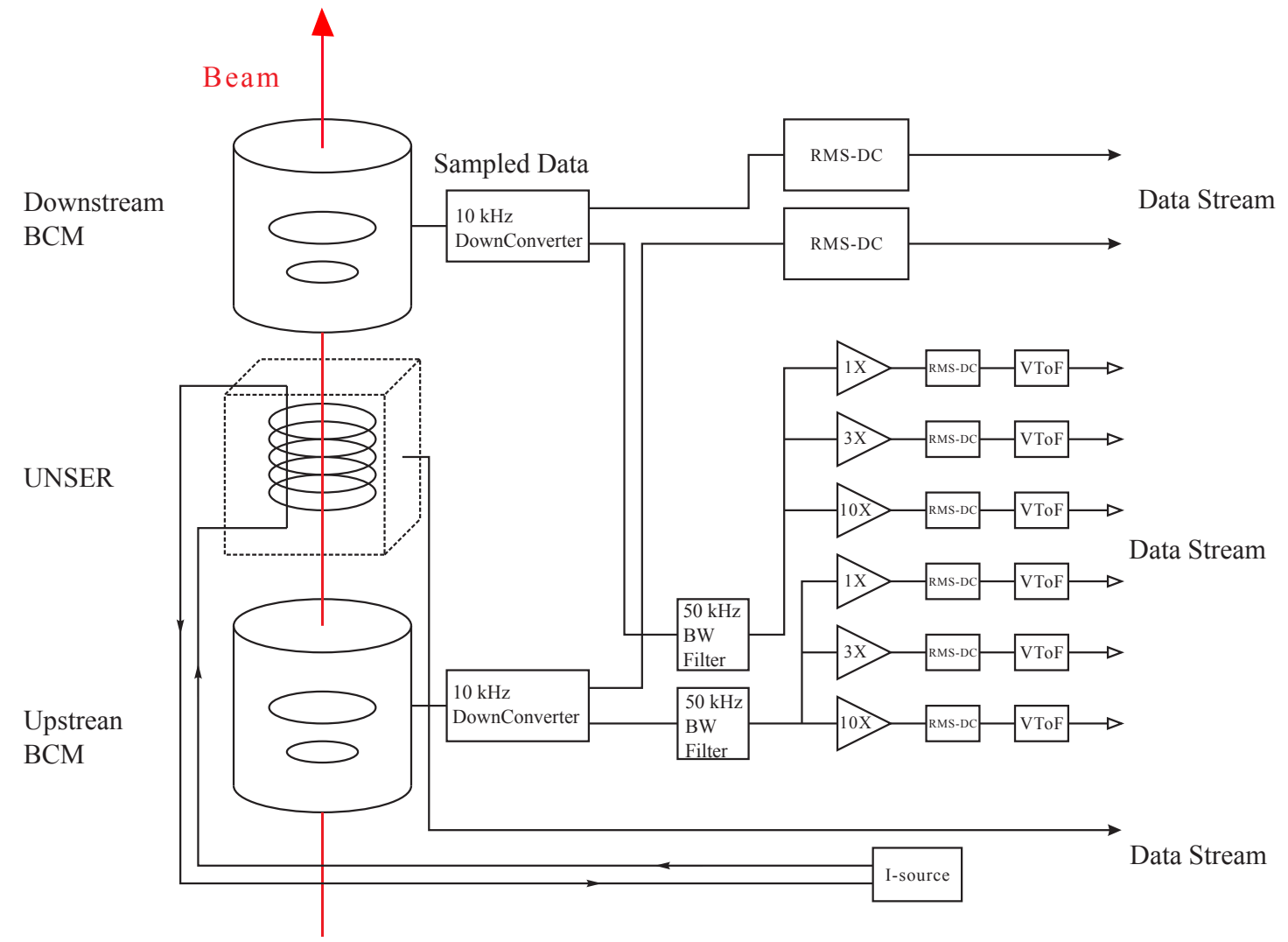

Figure 4.5: Schematic of the Hall A beam current monitors [52]. Two BCMs are used for the continuous monitoring of the beam current; they can be absolutely calibrated by the Unser. Measurements are recorded in the data stream as a part of Experimental Physics and Industrial Control System (EPICS) information (sampled data) and as a scaler information (integrated data). 
SuperHarps adjacent to each of the BPMs. The SuperHarps perform an invasive measurement of the beam position by scanning three thin wires: one vertically oriented and two at $\pm 45^{\circ}$.

\subsubsection{Beam Raster}

Experiment E05-015 used a high pressure glass target cell, in which the pressure can rise to $\sim 14$ atm under running conditions (shown in chapter 5). The high intensity electron beam tends to overheat and damage the target cell since the glass window has a thickness less than $0.2 \mathrm{~mm}$. In order to prevent this, a fast raster is used. The raster uses a pair of horizontal and vertical dipoles located $23 \mathrm{~m}$ upstream of the target. The system generates a rectangular raster pattern with a highly uniform distribution on the target. During the experiment, a $3 \mathrm{~mm}$ $\times 4 \mathrm{~mm}$ raster was used with the ${ }^{3} \mathrm{He}$ target cell or the reference cell (see chapter 5 as shown in Figure 4.6. The rastered beam was not used with carbon foils, since for these there is no overheating concern.

\subsection{High Resolution Spectrometers}

The main equipment of Hall $\mathrm{A}$ is the pair of almost identical High Resolution Spectrometers [49]. One is positioned on the left side of the beam line and is accordingly called Left HRS, while the other one is positioned on the right side of the beam line and called Right HRS. During E05-015, both spectrometers were configured to detect electrons in single-arm mode. The LHRS and RHRS data acquisition systems were synchronized during the experiment in order to increase the statistics and as a method to cross-check results. The basic layout of each spectrometer is shown in Figure 4.7. It comprises three quadrupole magnets and

one dipole magnet in a QQDQ configuration, with a central bending angle of $45^{\circ}$ in the vertical direction and an optical length of $23.4 \mathrm{~m}$. Table 4.2 shows the 


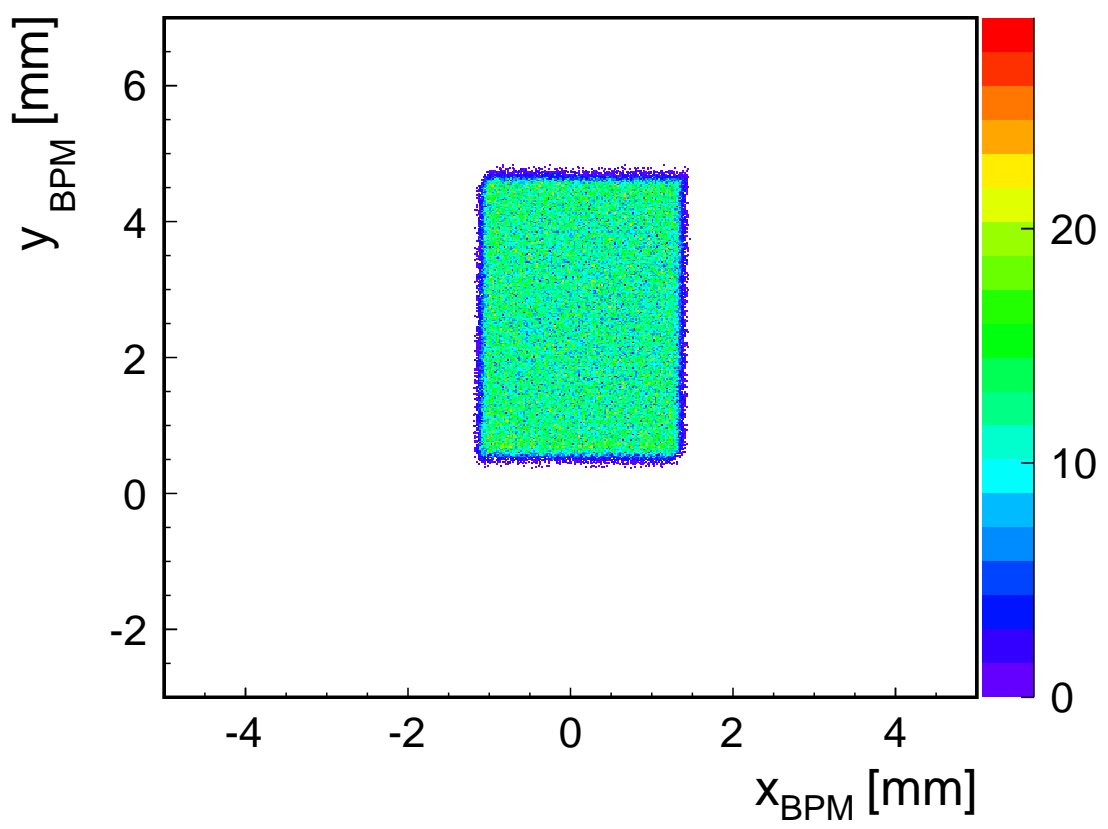

Figure 4.6: Beam position with beam raster on. Displayed is a $x$ and $y$ position of the beam from the BPM while the raster was on.

main characteristics of LHRS and RHRS.

\subsubsection{Detector Package}

The HRS detector package, together with the data acquisition (DAQ) electronics, is located inside a shielded hut at the top of the spectrometer. The shielded hut is made of $10 \mathrm{~cm}$ thick steel frame with a $5 \mathrm{~cm}$ lead layer inside and a layer of concrete outside [49]. It protects the detectors and DAQ electronics from the radiation background in the Hall. The detector package for experiment E05-015 consists of the following detector components (Figure 4.8):

- A pair of Vertical Drift Chambers (VDCs) to provide tracking information.

- Two scintillator planes (S1 and S2m) to provide timing information and the main trigger.

- A Cherenkov detector for particle identification. 


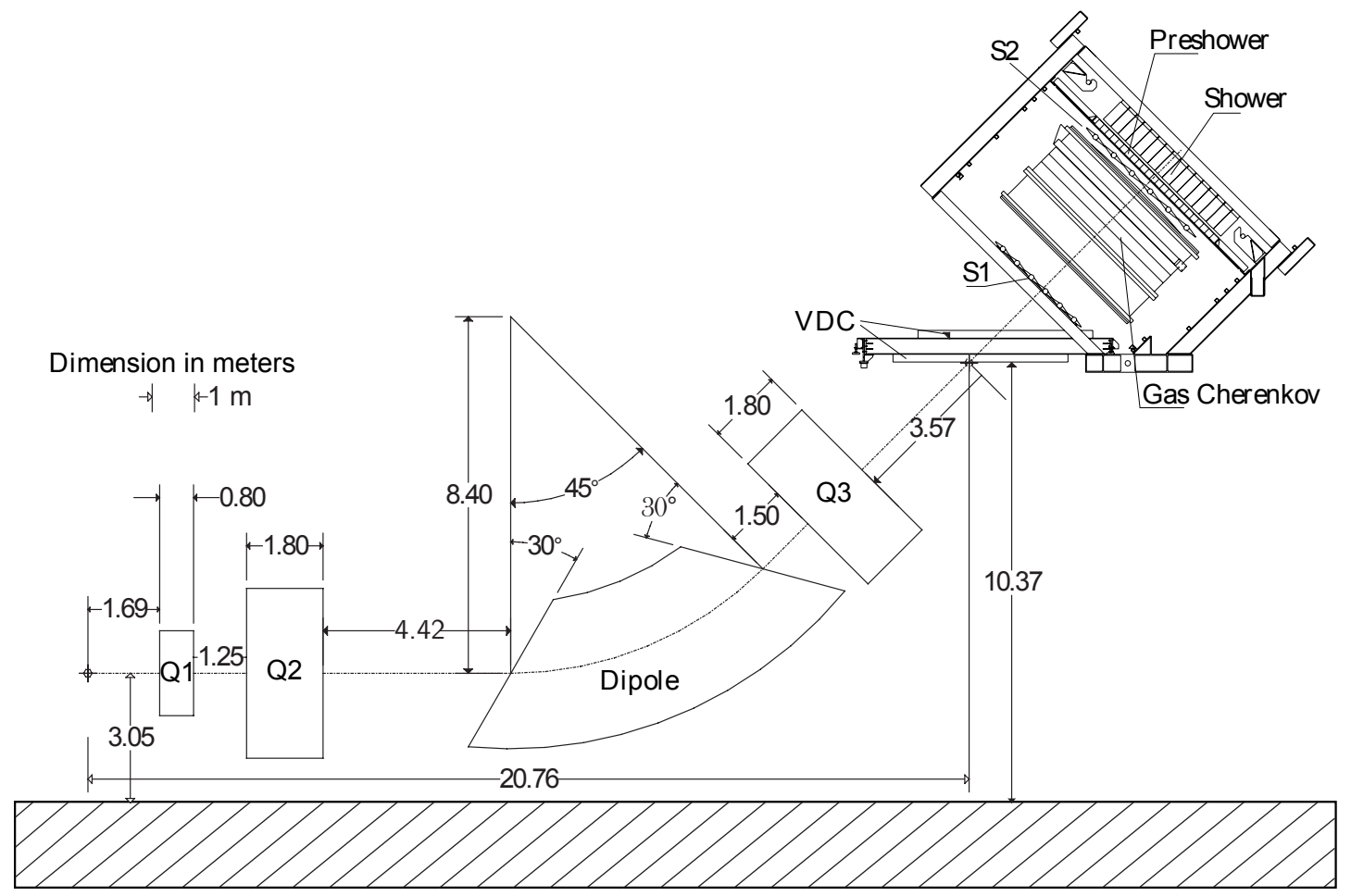

Figure 4.7: HRS layout [53]. The HRS uses three quadruple magnets, Q1, Q2 and Q3, and one dipole magnet, D, to transport particles into the detector package. The detector package for experiment E05-015 consisted of two trigger scintillator planes, S1 and S2, a gas Cherenkov detector, and two lead-glass calorimeters called pion rejector 1 and pion rejector 2 for left HRS, pre-shower and shower for right HRS. 
Table 4.2: Main characteristic of the Hall A high resolution spectrometers [49]. The resolution values are given in terms of Full Width at Half Maximum (FWHM).

\begin{tabular}{|c|c|}
\hline Configuration & QQDQ vertical bend \\
Bending Angle & $45^{\circ}$ \\
Optical Length & $23.4 \mathrm{~m}$ \\
Momentum Range & $0.3-4.0 \mathrm{GeV} / c$ \\
Momentum Acceptance & $-4.5 \% \leq \delta p / p \leq 4.5 \%$ \\
Momentum Resolution & $2.0 \times 10^{-4}$ \\
Angular Range & $12.5^{\circ}-150^{\circ}$ \\
Horizontal Angular Acceptance & $\pm 30 \mathrm{mrad}$ \\
Vertical Angular Acceptance & $\pm 60 \mathrm{mrad}$ \\
Horizontal Angular Resolution & $0.5 \mathrm{mrad}$ \\
Vertical Angular Resolution & $1.0 \mathrm{mrad}$ \\
Central Solid Angle & $6 \mathrm{msr}$ \\
Transverse Length Acceptance & $\pm 5 \mathrm{~cm}$ \\
Transverse Position Resolution & $1 \mathrm{~mm}$ \\
\hline
\end{tabular}

- Two sets of lead-glass counters (Pion-Rejector 1 and Pion-Rejector 2 for LHRS, Pre-shower and Shower for RHRS) to provide additional information on particle identification.
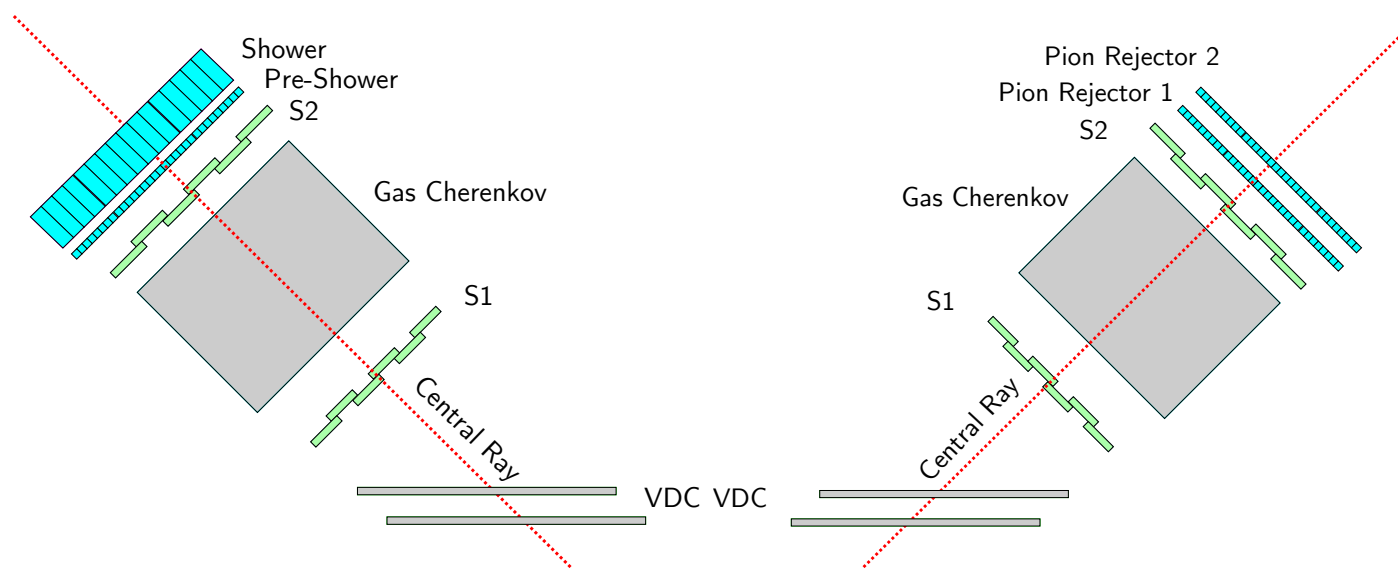

Figure 4.8: Illustration of the left and right HRS detector packages. 


\subsubsection{Vertical Drift Chambers}

The Vertical Drift Chambers are used to provide tracking information for the scattered particles [54]. Their ability to reconstruct the particle's trajectory at the focal plane is $\sigma_{x, y} \approx 100 \mu \mathrm{m}$ (spatial resolution) and $\sigma_{\theta, \phi} \approx 0.5 \mathrm{mr}$ (angular resolution). There are two VDCs in each of the spectrometers, and each of them is composed of two wire planes in the standard UV configuration. The wires in the $\mathrm{U}$ and $\mathrm{V}$ planes are perpendicular to each other and lie in the laboratory horizontal plane. Both the planes are oriented at $45^{\circ}$ with respect to the nominal particle trajectory. The distance between the two VDC planes is $335 \mathrm{~mm}$ and the separation between each pair of $\mathrm{U}$ and $\mathrm{V}$ planes is $26 \mathrm{~mm}$ as shown in the Figure 4.9. There are a total of 368 sense wires in each plane, which are spaced $4.24 \mathrm{~mm}$ apart. The wires are made of Au-plated tungsten, and each wire plane is sandwiched between two cathode planes. The chambers are filled with a gas mixture of argon (62\%) and ethane (38\%). A $4.0 \mathrm{kV}$ high voltage is applied to produce the electric field. For a typical track, the charged particle passes through the chamber at an angle of about $45^{\circ}$ and produces electron and ion pairs along its pass. The ionized electrons drift along the electric field at a velocity of $\sim 50$ $\mu \mathrm{m} / \mathrm{ms}[54]$ and fire an average of five sensor wires. The drift distance is calculated using the drift time, which is measured using time-to-digital converters (TDCs). The cross-over point at which the track passes through the sense-wire plane is determined using a linear fit of the drift distance.

\subsubsection{Scintillator Planes and Trigger Electronics}

There are two scintillator planes in both LHRS and RHRS [49], S1 and S2m. These planes are used to form the triggers specific to the requirements of the experiment.

The S1 plane consists of six scintillator paddles with two Photo-Multiplier Tubes 

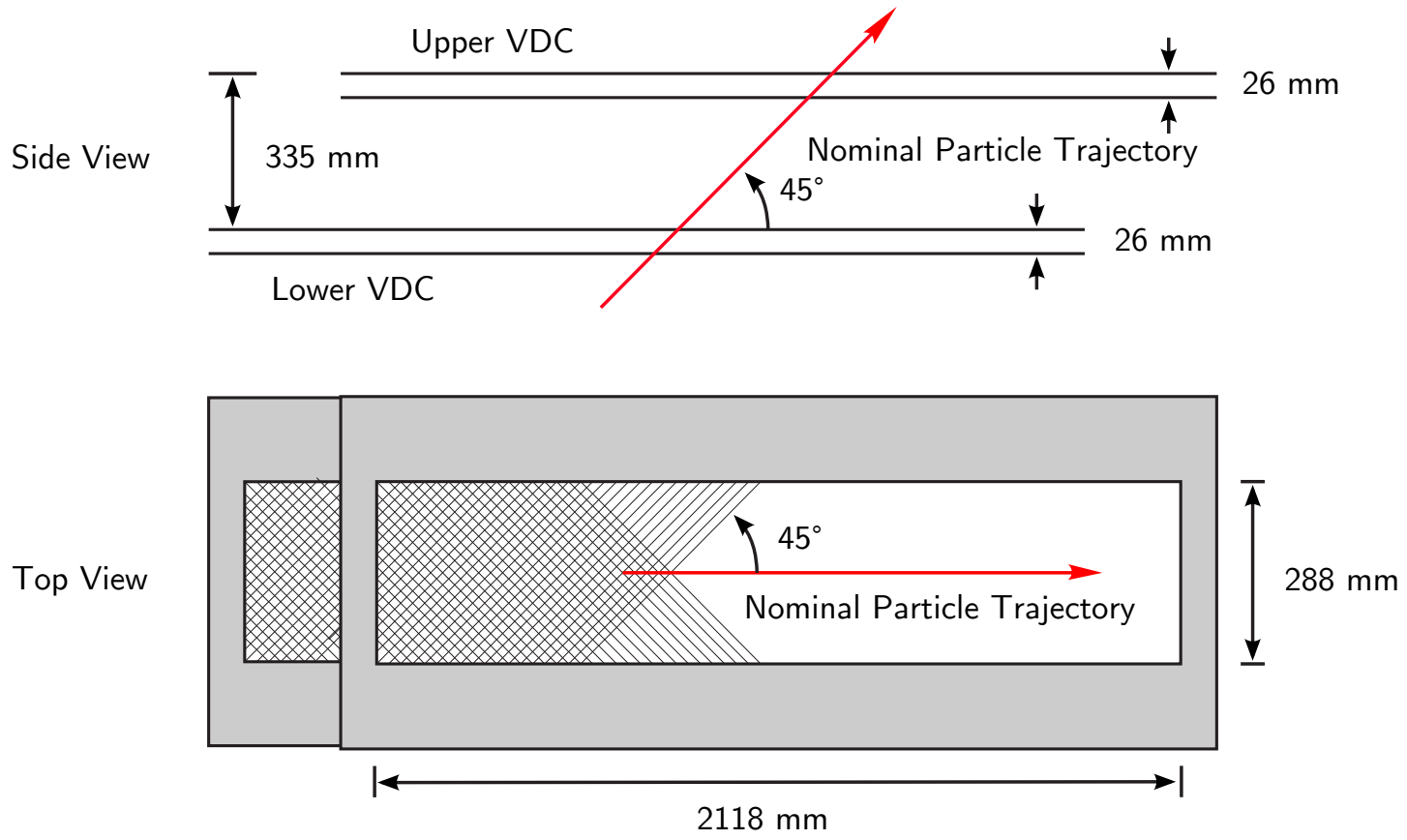

Figure 4.9: Top and side view of VDCs [54]. The size of the rectangular aperture of each chamber is $2118 \mathrm{~mm} \times 288 \mathrm{~mm}$. Each chamber consists of two orthogonal planes of wires ( $\mathrm{U}$ and $\mathrm{V}$ ) that are inclined at an angle of $45^{\circ}$ with respect to the spectrometer's dispersive direction. The VDCs lie in the laboratory horizontal plane. 


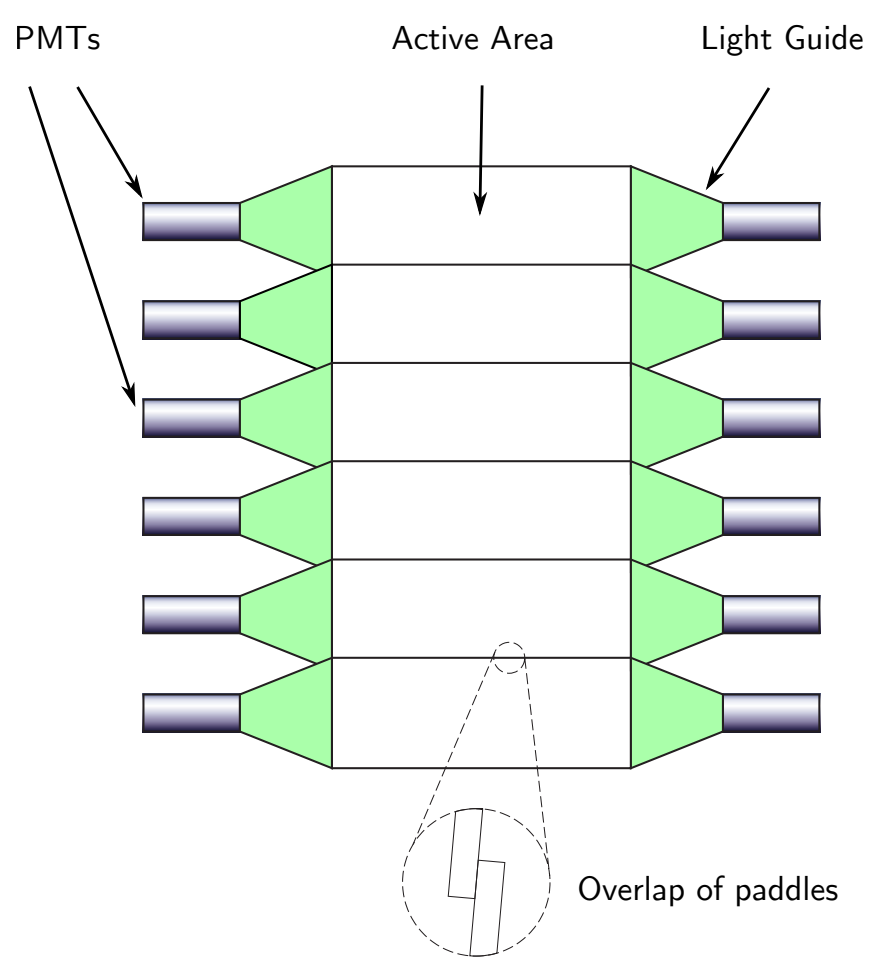

Figure 4.10: Layout of the S1 scintillator counter [55]. The paddles overlap by 10 mm.

(PMTs) on each paddle with one at each end of the paddle. The active volume of each paddle is $36 \mathrm{~cm}$ (length) $\times 29.3 \mathrm{~cm}$ (width) $\times 0.5 \mathrm{~cm}$ (thickness). As shown in Figure 4.10, the paddles are installed at a small angle with respect to the $\mathrm{S} 1$ plane and overlap by $10 \mathrm{~mm}$ each other. $\mathrm{S} 1$ is designed to be thin in order to minimize particle absorption and it provides the first component of the main trigger.

The S2m plane is formed by 16 scintillator paddles with two PMTs reading out both ends of each paddle. Each scintillator paddle has a dimension of $43.2 \mathrm{~cm}($ length $) \times 14 \mathrm{~cm}($ width $) \times 5.08 \mathrm{~cm}$ (thickness). Unlike S1, the paddles do not overlap. Besides providing input to the trigger, S2m also provides the timing information. 


\subsubsection{Gas Cherenkov Detector}

The Gas Cherenkov counter is used to distinguish electrons from other particles. It is one of the most efficient particle identification detectors. In our case, the detector was used to separate electrons from pions. The design and construction of the detector for JLab was completed by INFN and Saclay [56]. The operation of the detector is based on the Cherenkov effect. The speed of light in a medium is:

$$
c_{\text {medium }}=c_{0} / n
$$

where $c_{0}$ is the speed of light in vacuum and $n$ is the refractive index of the medium. When a particle passes through a medium with a speed larger than the speed of light in that medium, Cherenkov radiation is emitted. The threshold velocity can be transformed into the minimal particle momentum required for a particular particle to emit Cherenkov light:

$$
p_{\text {threshold }}=\frac{m c_{0}}{\sqrt{n^{2}-1}}
$$

It can be seen that the particle mass and refractive index determine the threshold momentum. To meet the needs of the specific experiment, one uses media chosen for appropriate refractive indices to generate light for the selected particle types.

The Hall A gas Cherenkov detectors are filled with $\mathrm{CO}_{2}$ gas under atmospheric pressure, with a refractive index $n=1.00041$. This sets the threshold momentum for electrons to $0.017 \mathrm{GeV} / c$, for pions to $4.8 \mathrm{GeV} / c$, and for protons to $32 \mathrm{GeV} / c$. Within the momentum acceptance of the HRSs, only electrons can emit Cherenkov light, which allows us to be able to distinguish them from other particles. The HRS Chrenkov detectors are positioned between the S1 and S2m plates. The detectors are $1 \mathrm{~m}$ long with a $250 \mathrm{~cm} \times 80 \mathrm{~cm}$ wide surface area. As shown in Figure 4.11, each Cherenkov has 10 spherical mirrors arranged as a 2 (transverse) $\times 5$ (dispersive) array. 

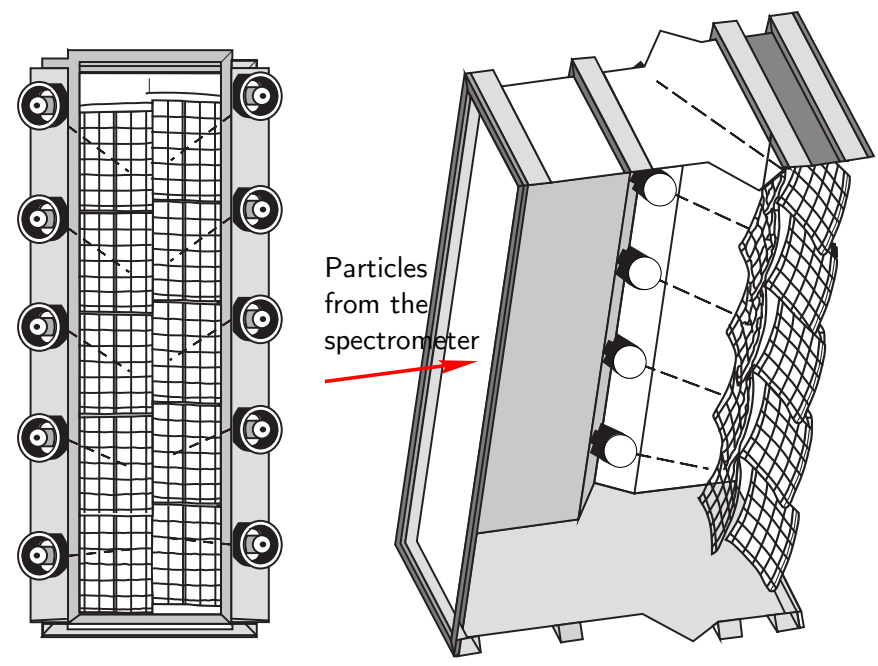

Figure 4.11: Cherenkov detector schematic [56].

Although pions do not directly produce any Cherenkov light in the detector, they can still interact with the atoms in the detector and create secondary electrons, which can emit Cherenkov light and produce signals in the analog-todigital converters (ADCs). But the secondary electrons do not necessarily move in the same direction as the scattered electrons, and therefore the emitted light is not efficiently collected by the mirrors. The summed ADC signals for the secondary electrons are mostly at the single photo-electron peak. On the other hand, the scattered electrons emit Cherenkov light producing multiple photo-electrons. During the experiment, the average number of photo-electrons for each PMT was $\sim 7$.

\subsubsection{Lead Glass Counters}

Two sets of lead glass counters are used for providing additional information for particle identification [49]. They are often referred to as the pion rejector layer 1, layer 2 for LHRS and pre-shower, shower for RHRS. Together with the Gas Cherenkov detector, the lead glass helps in separating electrons from pions. The principle of the lead glass counter is that when an energetic particle passes through dense material, it can generates cascades of photons and $e^{+}-e^{-}$pairs, 


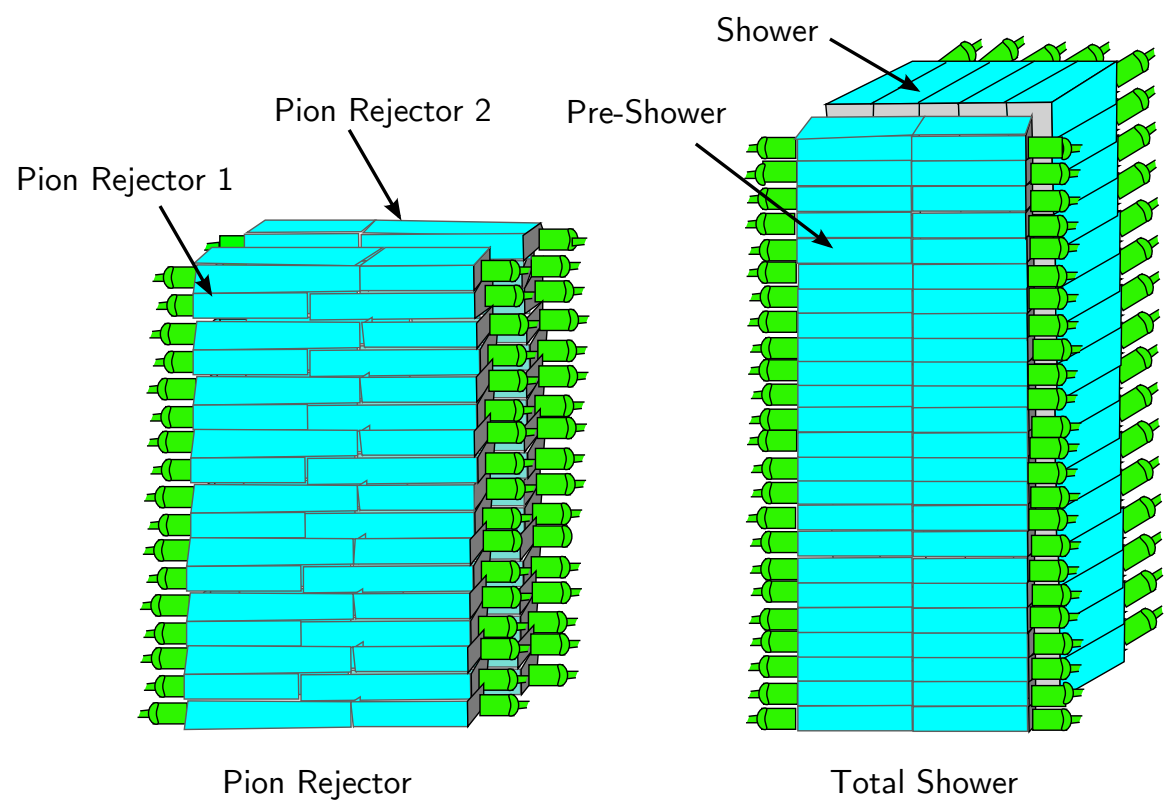

Figure 4.12: Configuration of electromagnetic calorimeters.

which generate more photons. The emitted photons can be detected by PMTs. Electrons develop a large signal due to an electromagnetic shower in the leadglass, whereas the pions do not develop signals as strong as the electrons because of their comparatively longer mean free path.

For the LHRS, the construction of both layers of the pion rejector is the same. Each layer is composed of 17 short blocks and 17 long blocks of lead glass, forming a 2 (transverse) $\times 17$ (dispersive) array. This is shown in Figure 4.12 left side. Therefore, both the layers are composed of 34 blocks and each block has a dimension of $30(35) \mathrm{cm}($ length $) \times 14.5 \mathrm{~cm}($ width $) \times 14.5 \mathrm{~cm}$ (thickness). The gap between two blocks in the first layer is covered by the blocks in the second layer. For the RHRS, pre-shower layer consists of two columns, each with 24 lead glass blocks with dimensions of $35 \mathrm{~cm}$ (length) $\times 10 \mathrm{~cm}$ (width) $\times 10 \mathrm{~cm}$ (thickness). The shower layer is composed of five columns each with 16 blocks with dimensions of $15 \mathrm{~cm}$ (length) $\times 15 \mathrm{~cm}$ (width) $\times 35 \mathrm{~cm}$ (thickness) 


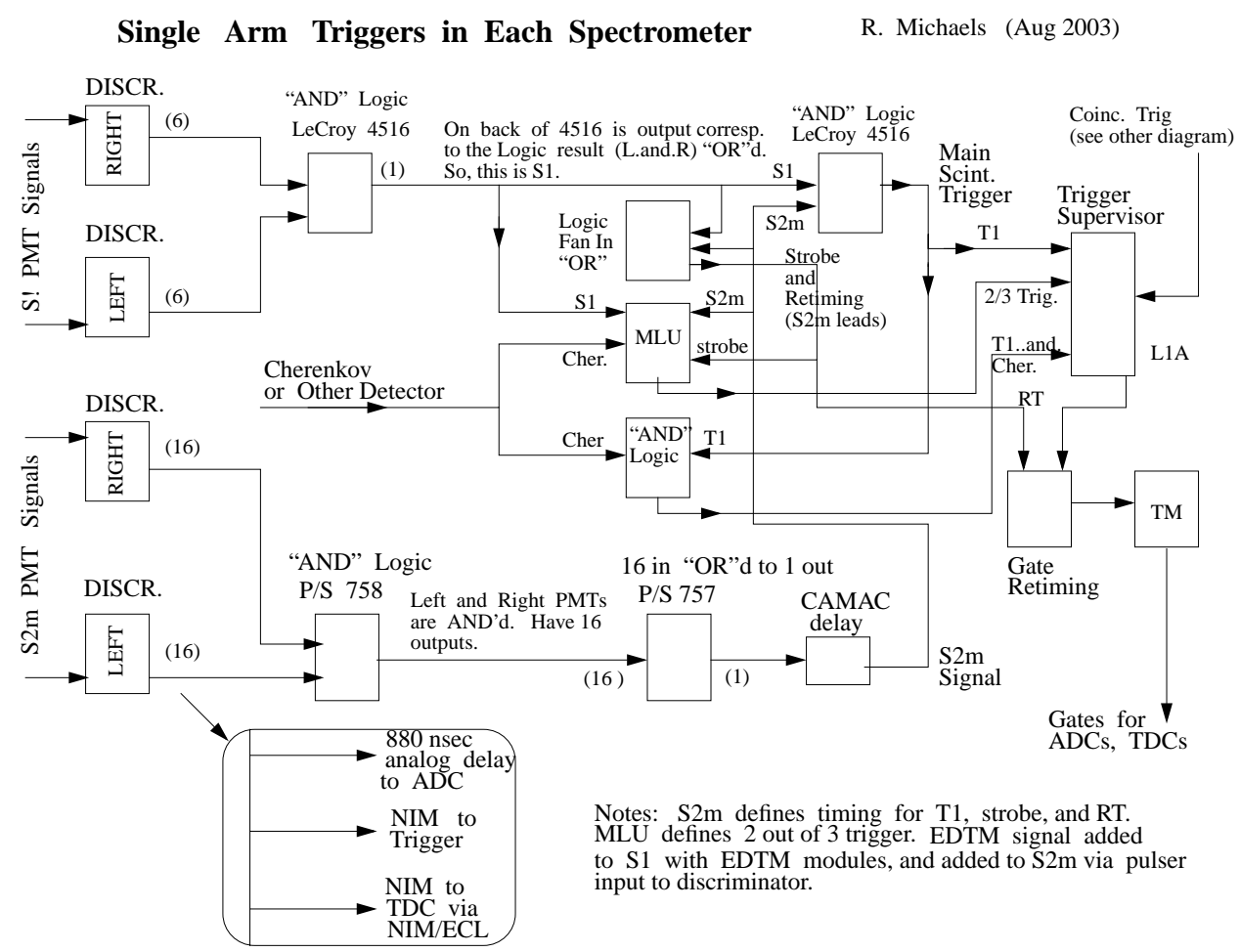

Figure 4.13: HRS single arm trigger scheme.

\subsubsection{Trigger System}

Table 4.3 shows triggers for E05-015. The Hall A system generally uses up to eight different triggers. They are electronic pulses, which are formed when a particle hits one or more detectors of the spectrometer(s). From the combination of these signals, one can decide whether they correspond to a certain physical process, and whether they should be recorded or not. Figure 4.13 shows the form of the HRS main trigger (T1/T3).

\subsubsection{Scaler Modules}

The raw signals from the PMTs on different detectors, as well as from the Beam Current Monitors (BCM), are counted by the scalers. For E05-015, depending upon the helicity states of the incoming electron beam and the target spin, five 
Table 4.3: Triggers used during E05-015.

\begin{tabular}{|c|c|}
\hline Trigger Type & Description \\
\hline T1 & RHRS singles (S1 and S2m) \\
T2 & RHRS efficiency (S1/S2m/Gas Cherenkov) \\
T3 & LHRS singles (S1 and S2m) \\
T4 & LHRS efficiency (S1/S2m/Gas Cherenkov) \\
T5 & - \\
T6 & - \\
T7 & - \\
T8 & Pulser $(1024 \mathrm{~Hz}$ clock $)$ \\
\hline
\end{tabular}

scaler modules were configured [52]: four scalers were gated as $(++),(+-),(-$ $+)$ and (- -), where the first quantity is the target spin state and the second one is the beam helicity. The last one was not gated by beam helicity or target spin state and was referred to as the ungated scaler. These five scalers were also gated with the run gate, i.e., they counted only when the run was started and stopped counting as soon as the run was ended. 


\section{Chapter 5 \\ Polarized ${ }^{3} \mathrm{He}$ Target}

Polarized ${ }^{3} \mathrm{He}$ can be used as an effective neutron target. The free neutron lifetime is very short, approximately $881 \mathrm{~s}$ [57]. Hence, a stable high-density neutron target is unavailable. Physicists have instead used light nuclei such as deuterium or ${ }^{3} \mathrm{He}$ to study the properties of neutron. The ${ }^{3} \mathrm{He}$ nucleus is composed of two

protons and one neutron. The ground state of the ${ }^{3} \mathrm{He}$ wave function is dominated by the S-state [58] [59], in which the two protons are anti-aligned, so to first order the spin direction of the ${ }^{3} \mathrm{He}$ nucleus depends on the neutron spin direction. Therefore, a polarized ${ }^{3} \mathrm{He}$ target is an effective polarized neutron target.

\subsection{Spin-Exchange Optical Pumping (SEOP)}

The level splitting of ${ }^{3} \mathrm{He} 1 \mathrm{~S}$ and $2 \mathrm{P}$ states is very large, and existing lasers are not able to provide enough power to polarize ${ }^{3} \mathrm{He}$. Thus the direct polarization of dense ${ }^{3} \mathrm{He}$ targets is not feasible. Only through indirect methods, such as spinexchange optical pumping, can these targets be polarized. The spin-exchange optical pumping mechanism can be divided into two steps according to their time sequence. The first step is optically pumping alkali atoms to produce polarized electrons. The second step is for the polarized electrons to transfer their polarization to the ${ }^{3} \mathrm{He}$ nuclei by a spin-exchange interaction. 


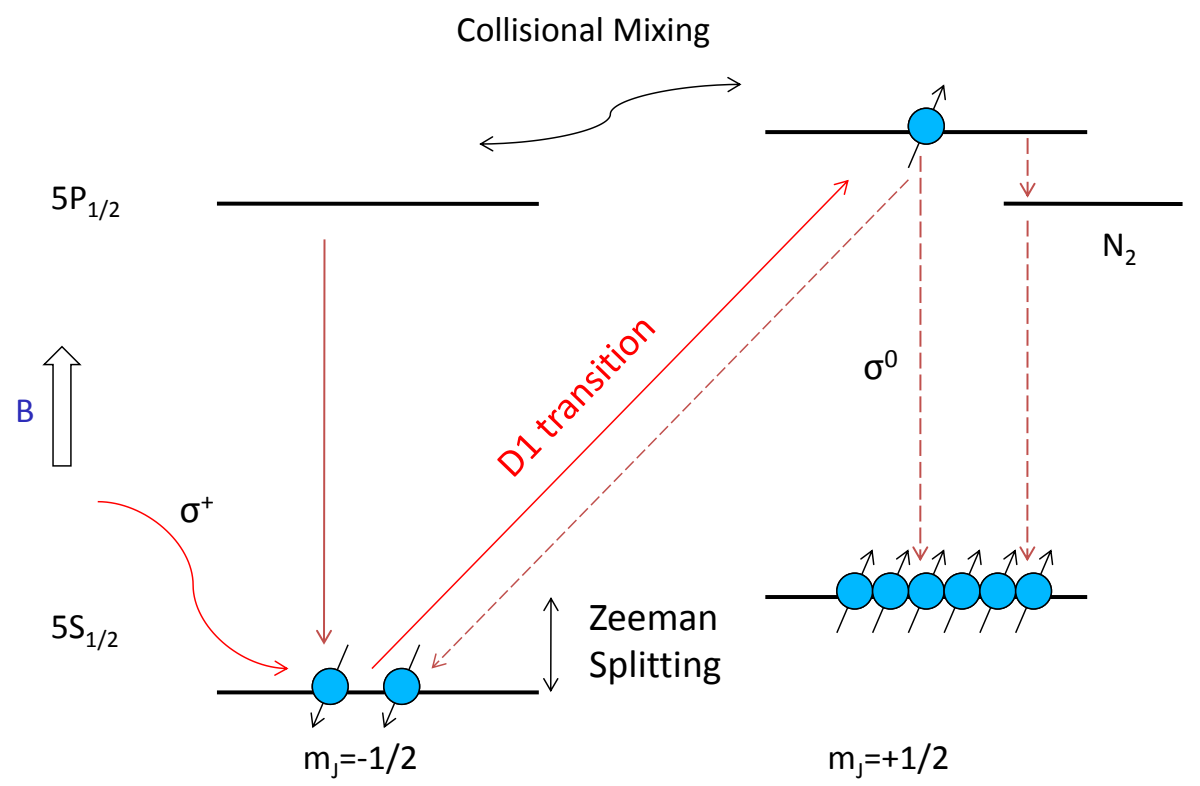

Figure 5.1: A diagram explaining optical pumping. (1)Rb atoms in a magnetic field are exposed to circularly polarized laser light. (2) The valence electron is excited from the $5 S_{1 / 2}$ to $5 P_{1 / 2}$ state. (3) The electron decays by emitting a photon into either the $m_{J}=-1 / 2$, where it repeats steps (2) and (3), or into the $m_{J}=+1 / 2$ where (4) it remains.

\subsubsection{Optical Pumping}

The polarized electrons are generated by optically pumping alkali atoms of ${ }^{85} \mathrm{Rb}$. ${ }^{85} \mathrm{Rb}$ has nuclear spin $I=5 / 2$, and the valence electron is in a $5 S_{1 / 2}$ ground state, with intrinsic spin $S=1 / 2$, angular momentum $L=0$, and total angular momentum $J=1 / 2$. When the alkali atoms are placed in a magnetic field, the valence ground state $5 S_{1 / 2}$ level will split into two sub-states, $m_{J}= \pm 1 / 2$. The number of alkali atoms with valence electrons in each of the two sub-states $n_{ \pm}$ obeys the normal Boltzmann distribution. For example, at room temperature $\mathrm{T}$ $\approx 300 \mathrm{~K}$, with magnetic field $\mathrm{B} \approx 30 \mathrm{G}$, the ratio of the alkali atoms in the two sub-states is $n_{+} / n_{-}=\exp \left(2 \mu_{e} B / k_{B} T\right) \approx 1.00001$; the number of alkali atoms in 
the two sub-states are substantially equal. In order to polarize alkali electrons, one must find a way to increase the number of alkali atoms in one state (e.g., $m_{J}=+1 / 2$ ), while reducing the number of atoms in the other state (e.g., $m_{J}=-$ 1/2). Optically pumping alkali atoms is a two-step process. (1) Assuming that the laser is left-hand circularly polarized $\left(\sigma^{+}\right)$, according to the selection rule $\Delta m_{J}=+1$, only electrons in $m_{J}=-1 / 2$ can absorb photons and be excited to $m_{J}=+1 / 2$ to be state $5 P_{1 / 2} ;(2)$ An electron in an excited state emits nonpolarized light $\left(\sigma^{0}\right)$, decaying to the state $5 S_{1 / 2}$. The probability of decay to the $m_{J}=-1 / 2$ and $m_{J}=+1 / 2$ states is $50 \%$ to each. After multiple cycles of excitation and decay, optical pumping can produce alkali atoms that are close to $100 \%$ in the state $5 S_{1 / 2}, m_{J}=+1 / 2$, as shown in Figure 5.1 .

\subsubsection{Spin Exchange}

In 1960, Bouchiat et al. [60] found that alkali atoms can transfer their electron polarization to ${ }^{3} \mathrm{He}$ nuclei. Binary collisions between atoms dominate in the angular momentum transfer, and the Hamiltonian can be expressed as [61]:

$$
\vec{H}(R)=\vec{H}_{S R}+\vec{H}_{S E}=\gamma(R) \vec{N} \cdot \vec{S}+\alpha(R) \vec{I} \cdot \vec{S}
$$

where $R$ is the distance between the two atoms. The first term of the formula represents the interaction between electron spin $\vec{S}$ and the angular momentum of ${ }^{3} \mathrm{He}$-alkali system, and the second term is the interaction between the electron spin $\vec{S}$ with the ${ }^{3}$ He nuclear spin $I$.

It was found that the spin-exchange efficiency for $\mathrm{K}$ is one order of magnitude higher than that of Rb [62] 63]. Even so, the available lasers could not provide enough power to polarize K electrons. A method of Hybrid Spin-Exchange Optical Pumping of ${ }^{3} \mathrm{He}$ was proposed [64] in order to achieve a high polarization. The hybrid alkali atoms are Rb and K. Polarized Rb atoms transfer their polarization to the $\mathrm{K}$ atoms, and finally to the ${ }^{3} \mathrm{He}$ nucleus, as shown in Figure 5.2 . The spin 


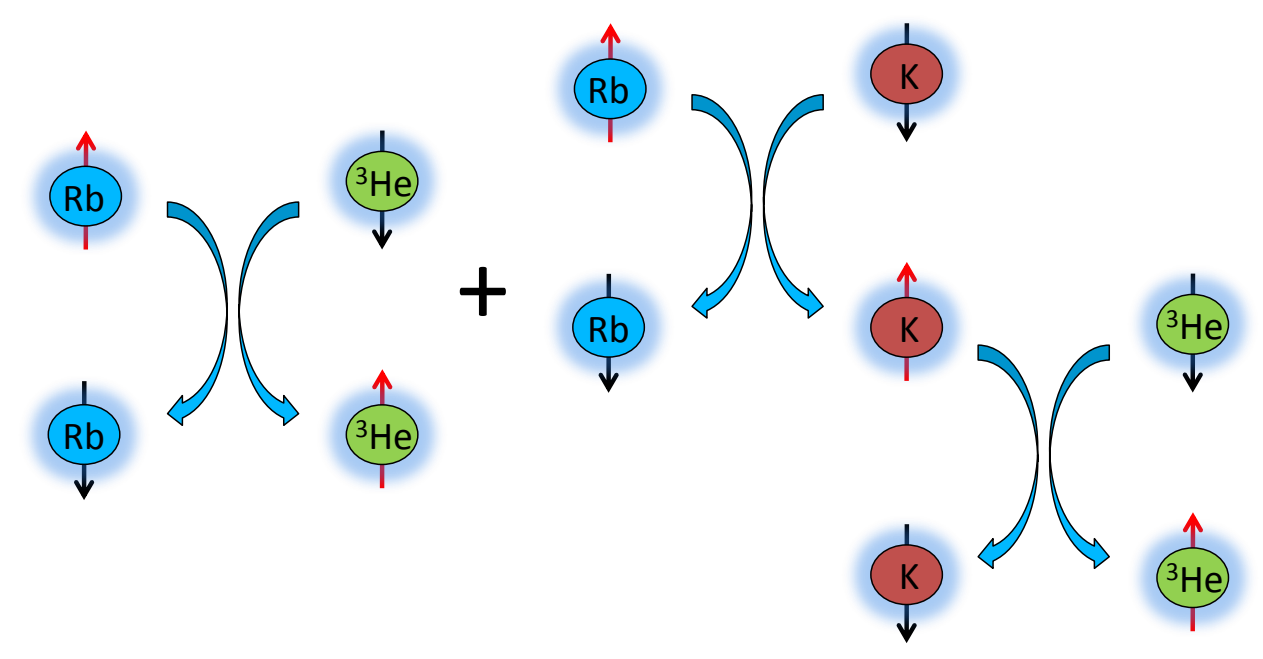

Figure 5.2: Spin exchange in a Rb-K hybrid cell.

exchange cross-section between $\mathrm{Rb}$ and $\mathrm{K}$ is pretty large, and the spin-exchange rate is much faster than the $\mathrm{Rb}$ spin-relaxation rates. Therefore, in a $\mathrm{Rb}-\mathrm{K}$ mixed system, $\mathrm{K}$ vapor has an electron polarization approximately equal to that of the $\mathrm{Rb}$ vapor.

\section{$5.2 \quad$ Target Setups}

A schematic of the E05-015 polarized ${ }^{3}$ He target set-up is shown in Figure 5.3. It contains (1) the target cell, including pumping chamber, transfer tube and target chamber (Section 5.2.1); (2) the laser system, including lasers and optical elements (Section 5.2.2); (3) the magnet system, including Helmholtz coils, RF coils and the power supplies (Section 5.2.3); (4) heating and temperature measurement system, including heating pipe, heaters and Resistive Temperature Devices (RTD) 


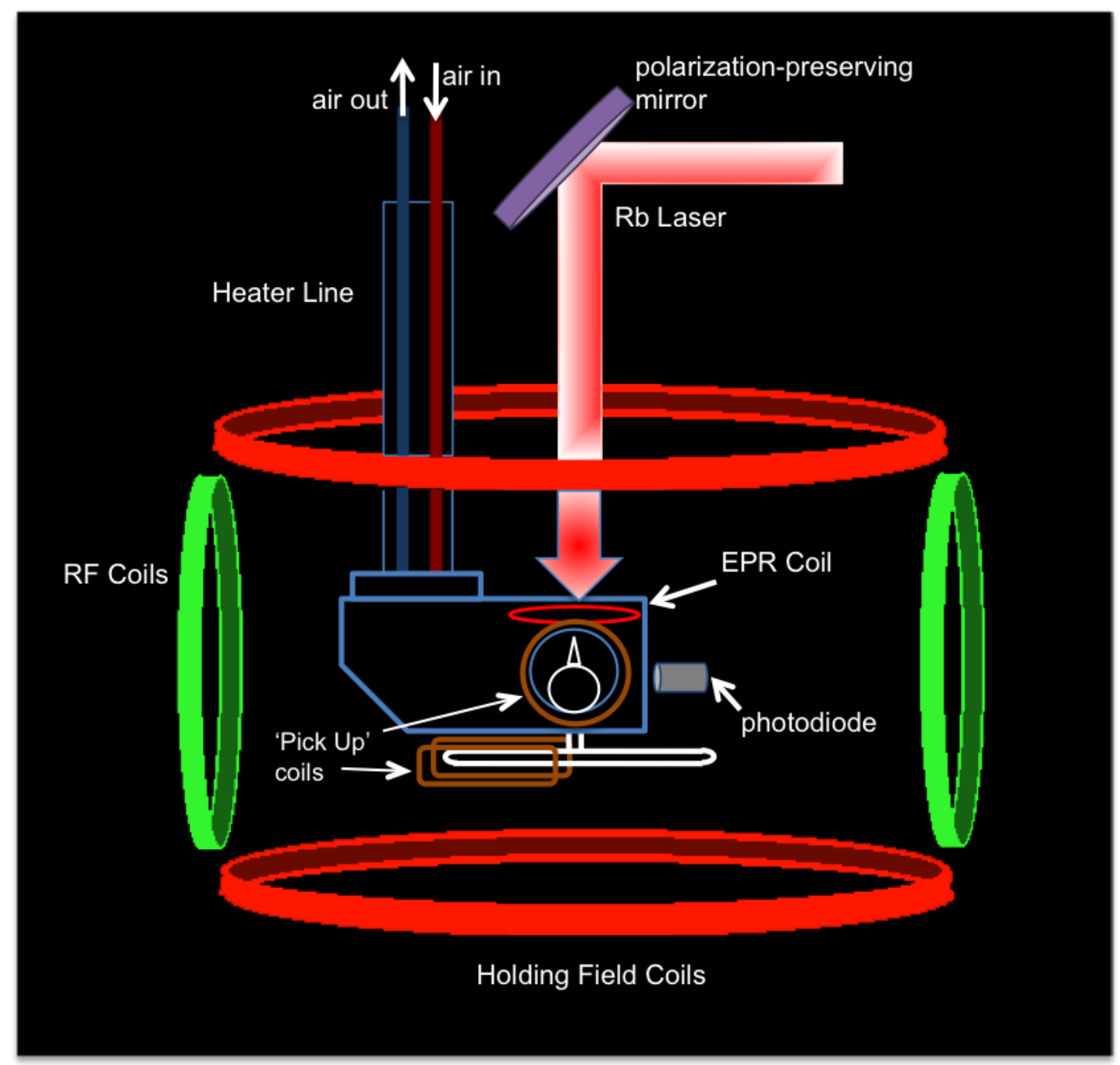

Figure 5.3: A schematic of the vertically polarized ${ }^{3} \mathrm{He}$ target [42]. Note that the Horizontal Helmholtz coils are not shown in this figure.

temperature sensors and temperature instruments (Section 5.2.4); (5) the NMR measurement system, including pick-up coils and the corresponding electronics system (Section 5.3); (6) the EPR measurement system, including the EPR coils, photodiode and the corresponding electronics system (Section 5.4).

\subsubsection{Characterizing the ${ }^{3} \mathrm{He}$ Cell}

The ${ }^{3} \mathrm{He}$ gas was contained in a hand-blown glass cell, which was made of GE180 aluminosilicate glass. Before the cell was sealed, it was filled with a small amount 
Table 5.1: Characteristics of ${ }^{3} \mathrm{He}$ cell used in E05-015.

\begin{tabular}{|c|c|}
\hline Name & Dominic \\
\hline Filled at & W\&M \\
\hline$V_{p c}\left(\mathrm{~cm}^{3}\right)$ & 183.1 \\
\hline$V_{t t}\left(\mathrm{~cm}^{3}\right)$ & 5.2 \\
\hline$V_{t c}\left(\mathrm{~cm}^{3}\right)$ & 74.2 \\
\hline$n_{0}$ (amagat) & 10.9 \\
\hline Lifetime (hour) & 20 \\
\hline $\mathrm{N}_{2}$ (amagat) & 0.125 \\
\hline
\end{tabular}

of $\mathrm{N}_{2}$ gas and a mixture of rubidium and potassium metal. The main characteristics of the cell used in this experiment are summarized in Table 5.1. The cell consists of the following three parts:

Pumping chamber (pc): a three-inch sphere with a typical wall thickness of $\approx$ $4 \mathrm{~mm}$. During the polarization process, the pumping chamber was heated to $\approx 270{ }^{\circ} \mathrm{C}$ in order to vaporize the alkali metal.

Transfer tube $(\mathbf{t t}): \mathrm{a} \approx 9 \mathrm{~cm}$ long tube connecting the pumping and target chambers. During the experiment, polarized ${ }^{3} \mathrm{He}$ gas diffused from the pumping chamber to the target chamber at a rate of around 1 cell volume per hour [52] through the transfer tube.

Target chamber (tc): a $40 \mathrm{~cm}$ long glass tube with an typical diameter of 1.9 cm. During the experiment, the electron beam passed through the glass windows and interacted with the polarized ${ }^{3} \mathrm{He}$ nuclei within the target chamber. The target chamber was cooled with ${ }^{4} \mathrm{He}$ cooling jets on both glass windows. During the experiment, the ${ }^{3} \mathrm{He}$ density in the target chamber was $\approx 14.8$ amagat ${ }^{1}$

\footnotetext{
${ }^{1} \mathrm{An}$ amagat is a density unit, 1 amagat is the gas density at $1 \mathrm{~atm}(=101.325 \mathrm{kPa})$ and 0 ${ }^{\circ} \mathrm{C}(=273.15 \mathrm{~K})$.
} 


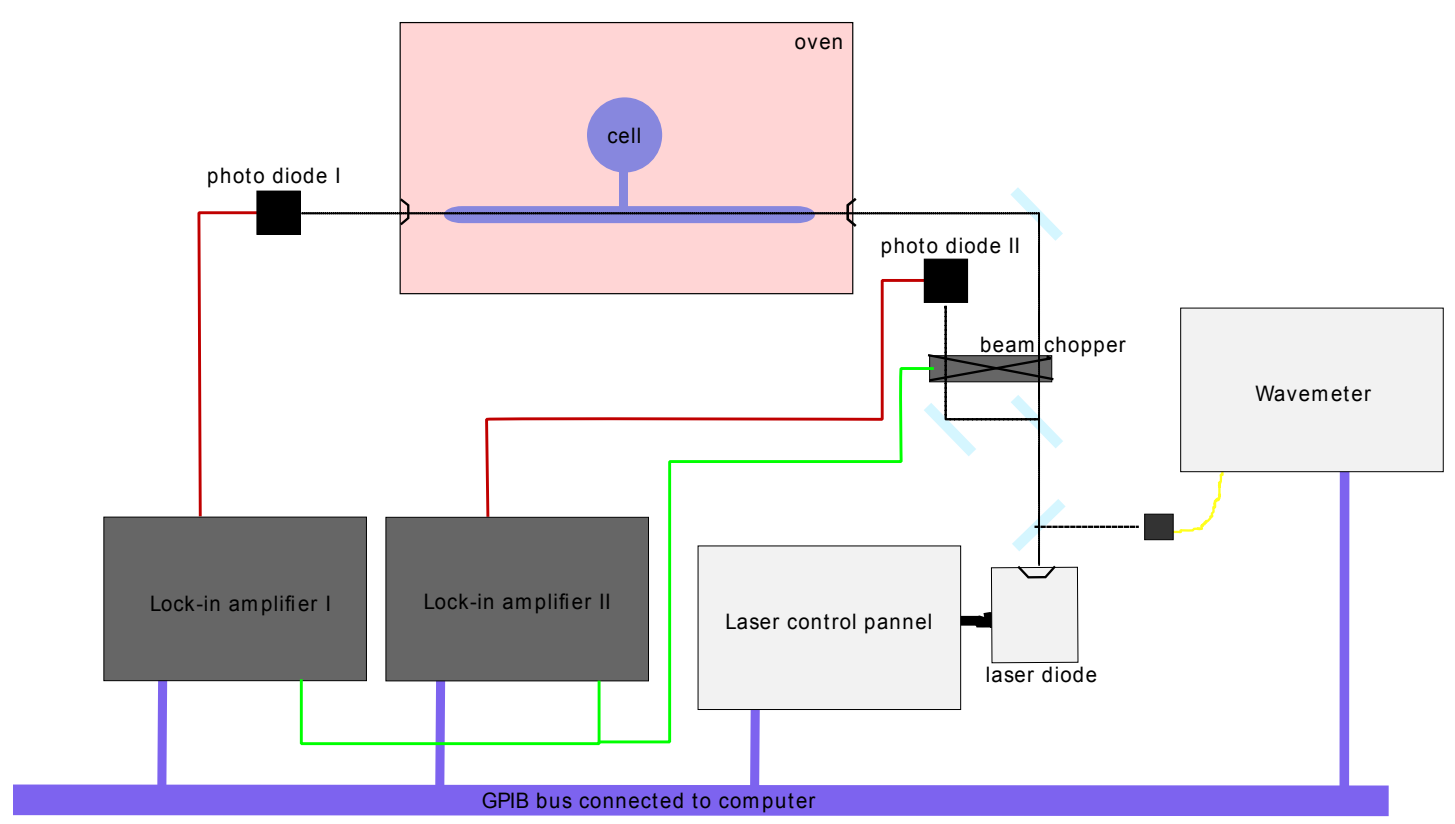

Figure 5.4: Setup for the pressure broadening measurement.

- Target Density: Knowledge of the ${ }^{3}$ He target density is crucial for the extraction of a target polarization and cross-sections. The target-cell density was measured by observing the collisional absorption broadening of the D1 and D2 lines of the alkali metal Rubidium ( $\mathrm{Rb})$ in the presence of ${ }^{3} \mathrm{He}$ gas, which acts as a high pressure perturbation of these lines. More details can be found in [65]. The instrumental setup that was used during these measurements is illustrated in Figure 5.4. The main equipment used in the density measurement was:

- One laser: New Focus Inc., $\lambda$ range: 790 nm - 801 nm; power: 0.1 mW $-6.5 \mathrm{~mW}$.

- Two lock-in amplifiers: Stanford Research System Inc., SR844-200 $\mathrm{MHz}$ RF lock-in amplifier.

- Two photo-receivers: New Focus, Inc. Large-area visible photo-receiver. Model 2031.

- One wave-meter: Burleigh Inc. WA-1100. 
Table 5.2: Parameters for the pressure broadening measurement.

\begin{tabular}{|c|c|c|}
\hline Parameters & Description & Setting Value \\
\hline Wavelength Start (nm) & Starting point of a wavelength scan & 793.30 \\
Wavelength Stop (nm) & Ending point of a wavelength scan & 796.30 \\
DC Motor Step Size (nm) & The incremental wavelength step & 0.15 \\
PZT Step Size (\%) & Percentage of a DC motor step & 2.5 \\
Average Data & Average data points per PZT setting & 6 \\
Filename & Accumulated data file & Arbitrary \\
Power (mW) & Diode Power & 6 \\
\hline
\end{tabular}

The laser beam goes through the two windows on the oven, and hits the photodiode. With the target cell placed inside the oven, the laser beam goes through the two windows of the target cell as well. A lens was placed before the photodiode to focus the laser after it went through the oven windows to efficiently collect the transmitted light. Both photodiodes were on low sensitivity to make sure they can read the signal. Generally we started the oven temperature from $65-80{ }^{\circ} \mathrm{C}$ (depending on the target cell), and took several scans $(10-15)$ at different temperatures. The temperature range should be at least $15{ }^{\circ} \mathrm{C}$ in order to make sure our density scans do not have a temperature dependence. However, in order to keep our Photo-Diodes within their linear range, the intensity of the signal should be no less than $0.1 \mathrm{mV}$, which prevented us from going to higher temperature. Parameters used in pressure broadening measurements are listed in Table 5.2. Figure 5.5 shows the scan result of the cell Dominic at $95{ }^{\circ} \mathrm{C}$.

The basic shape of the Rb D1 spectral profile has the form of a Lorentzian:

$$
S(\nu)=\frac{A\left[1+0.6642 \cdot 2 \pi T_{d}\left(\nu-\nu_{c}\right)\right]}{\left(\nu-\nu_{c}\right)^{2}+(\gamma / 2)^{2}}+B
$$

where $A$ and $B$ are fit parameters, $T_{d}$ is the collision time, $\nu$ is the frequency of the laser light, $\nu_{c}$ is the central value of the resonance, and $\gamma$ is the FWHM of the signal. There are two contributions to the measured width of the absorption lines: the main one comes from the broadening due to collisions 


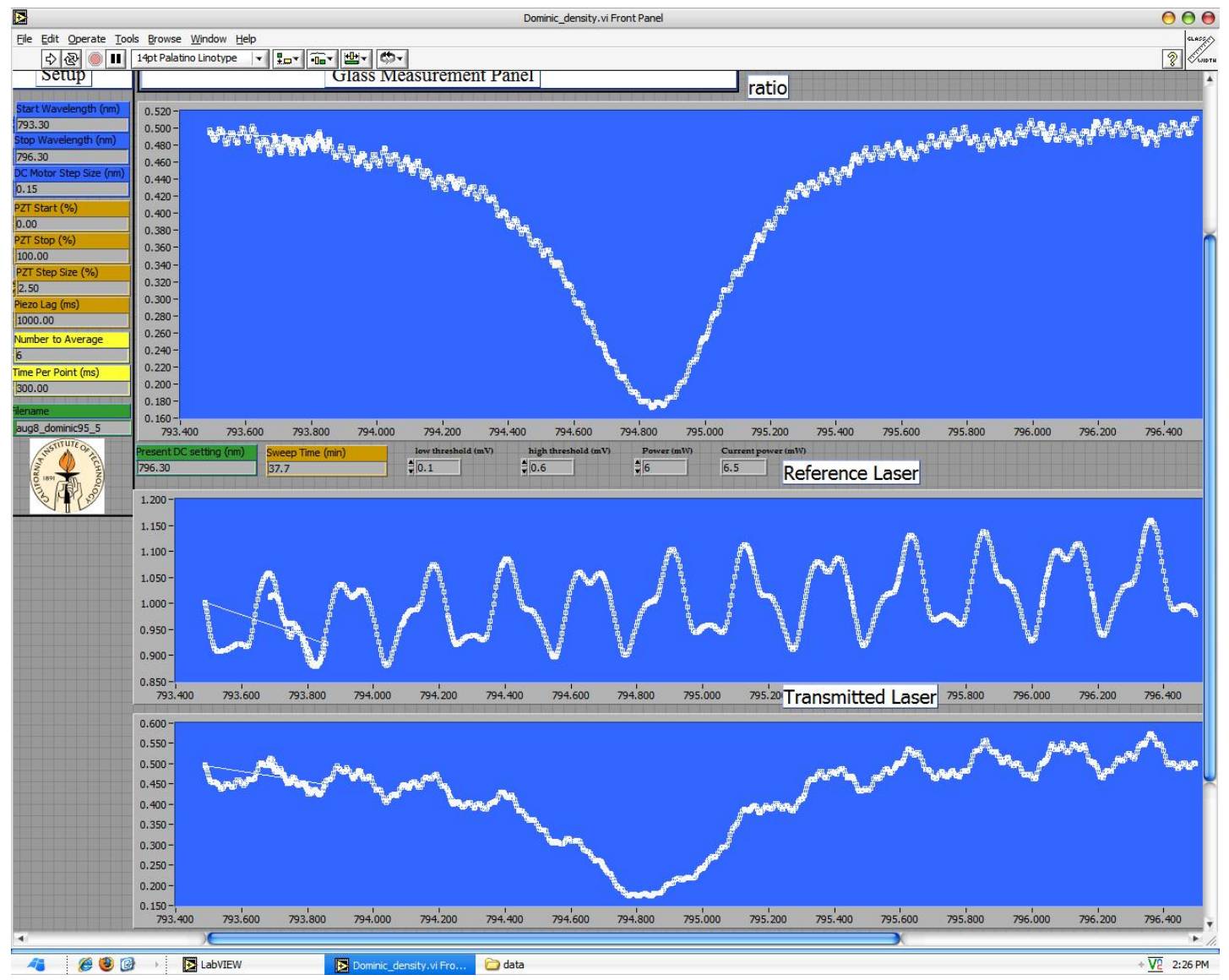

Figure 5.5: Illustration of the density scan figure for the cell Dominic at $95{ }^{\circ} \mathrm{C}$. 
Table 5.3: Pressure broadening coefficients [66].

\begin{tabular}{|c|cc|}
\hline Parameters & ${ }^{3} \mathrm{He}$ & $N_{2}$ \\
\hline D1 full width (GHz/amg) & $18.7 \pm 0.3$ & $17.8 \pm 0.3$ \\
D2 full width (GHz/amg) & $20.8 \pm 0.2$ & $18.1 \pm 0.3$ \\
\hline
\end{tabular}

with ${ }^{3} \mathrm{He}$. A roughly $1 \%$ contribution comes from the $N_{2}$ density, which is only about 0.1 amagat. The relevant proportionality constants as well as their temperature dependence are very well known; they are summarized in Table 5.3. In this calculation, one takes into account the temperature dependence of the constants which has the following form [66]: $T^{0.05 \pm 0.05}$ for a D1 width and $T^{0.53 \pm 0.06}$ for a D2 width. By measuring and fitting the absorption spectra, we found the ${ }^{3} \mathrm{He}$ density in the cell Dominic is $\approx 10.92$ amagat. More details on extracting the cell density from the line width can be found in [65].

- Wall Thickness: The objective of the wall thickness study is to determine the thickness of the target cell glass. During the experiment, the incoming and outgoing electrons lose energy when they pass through the cell windows and walls. Knowledge of the cell's thicknesses is mandatory to perform the radiative corrections (Section 6.8.1). In this study, several data scans were completed to measure the glass thickness of the polarized ${ }^{3} \mathrm{He}$ target and the reference cells. The cells that were used during the experiment were:

- Reference cell: GMB2

$-{ }^{3} \mathrm{He}$ cell: Dominic

When two or more coherent light sources interfere with each other, they produce a series of alternating bright or dark fringes as a result of their constructive or destructive interference, which depends on the relative phase of the interacting waves. The measurement of a transparent thin film thickness is conducted by detecting reflected laser light from the front and the back 


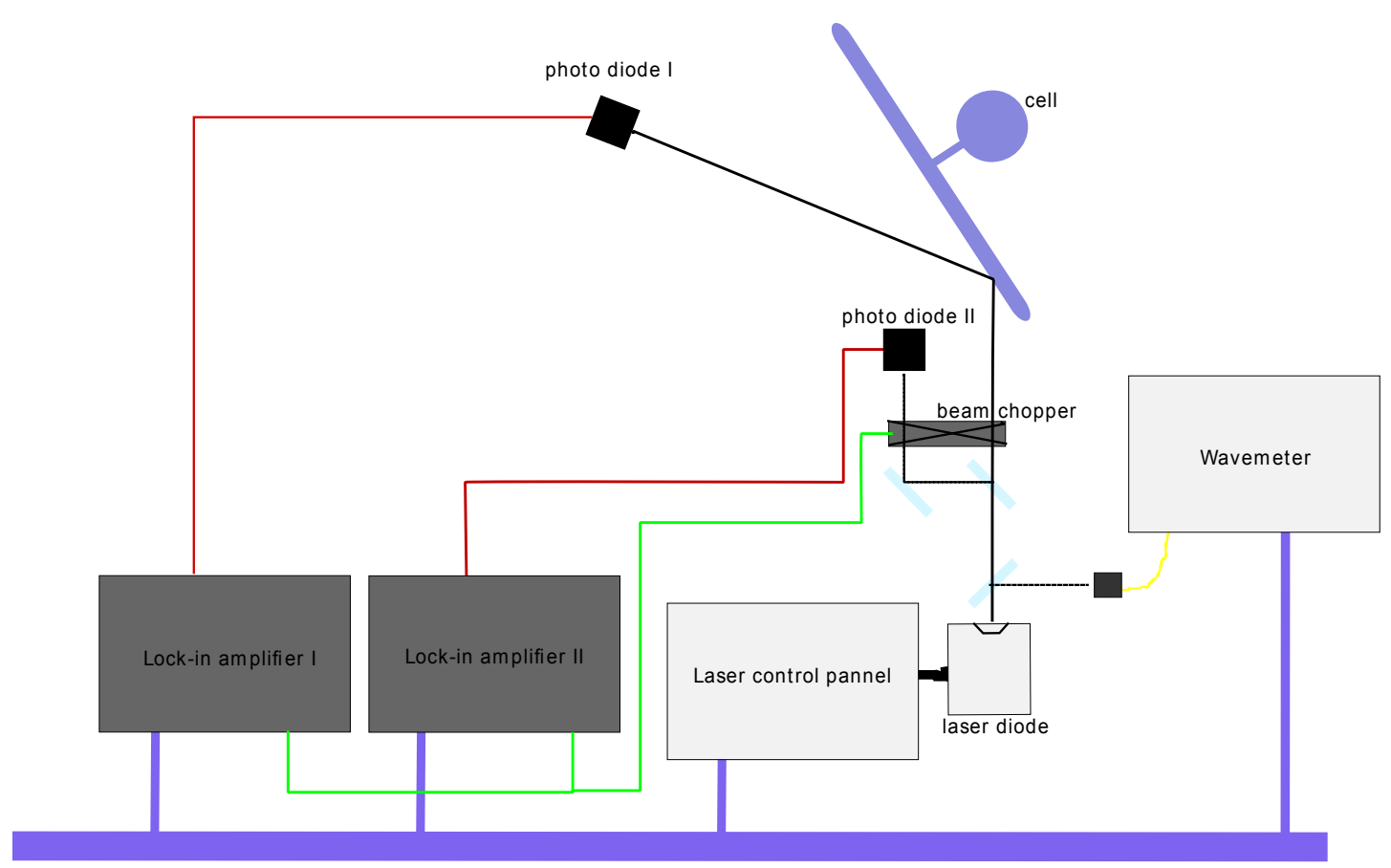

Figure 5.6: Setup for cell wall thickness measurement.

surface of the film. The incident laser scatters from the glass producing waves that reflect from the front surface and others that refract and then reflect from the back surface. A constructive or destructive interference occurs when the wave refracted from the back surface through the film and then interferes with the reflected wave from the front surface. The type of interference depends on the optical path length difference and hence, the relative phase of the interacting waves. The instrumental setup that was used during these measurements is illustrated in Figure 5.6. The instruments are the same as used in the density measurement, except for adding an iris and removing the oven. The tunable diode laser was used to produce a continuous laser beam. The laser light is split into two beam paths. The laser beam, as is shown in Figure 5.6, goes through a beam splitter that removes approximately $10 \%$ of the beam to be read by a wave-meter. The latter is used to measure the laser's wavelength. The second beam goes through the optical chopper to the second beam splitter. The optical 
chopper causes the laser signal to oscillate at $1.4 \mathrm{kHz}$, and thus, the lock-in amplifier can cleanly read the signal from background. Roughly $30 \%$ of the light from the second splitter is sent to the first photo-receiver. However, the other $70 \%$ is reflected off the cell glass into an optical iris, and then, to the second photo-receiver. The optical iris is used to help focus on the small interference pattern required by the measurement. The signal from the two photo-receivers is sent into two lock-in amplifiers to the computer, where it gets processed and analyzed by a LabView program.

The cell's orientation and position are shown in Figure 5.7, where W1 (W2) is the upstream (downstream) window. In the wall and windows thickness measurements, one needs to make sure that all the optical components and LabView program parameters are set correctly. The important parameters of this program are the Diode-Controller (DC) motor step size, the percentage of the DC motor step size (PZT) and the start-stop wavelength window. The DC step motor allows one to monitor the incremental wavelength step. It was set to $0.15 \mathrm{~nm}$ during the entire measurement. The second PZT parameter was changed from $5 \%$ to $15 \%$ between the wall and windows measurements. However, the start-stop wavelength window was varied respectively between $3 \mathrm{~nm}$ to $10 \mathrm{~nm}$ for each wall and window thickness scan. Using these settings, the scan of each measured cell's wall (window) point took roughly 15 - $16(17-18)$ minutes.

What makes this task tricky is the effort required to find a good interference image, either with an Infra-Red (IR) card or a scope before proceeding with any measurement. These two tools were used to make sure that the laser shines on the required glass point. Thus, a good tuning of the cell's position makes finding the interference spot much easier, especially if the cell is positioned in the transverse plan relative to the incident laser direction. However, a slight planar movement of the cell is mandatory to keep the 


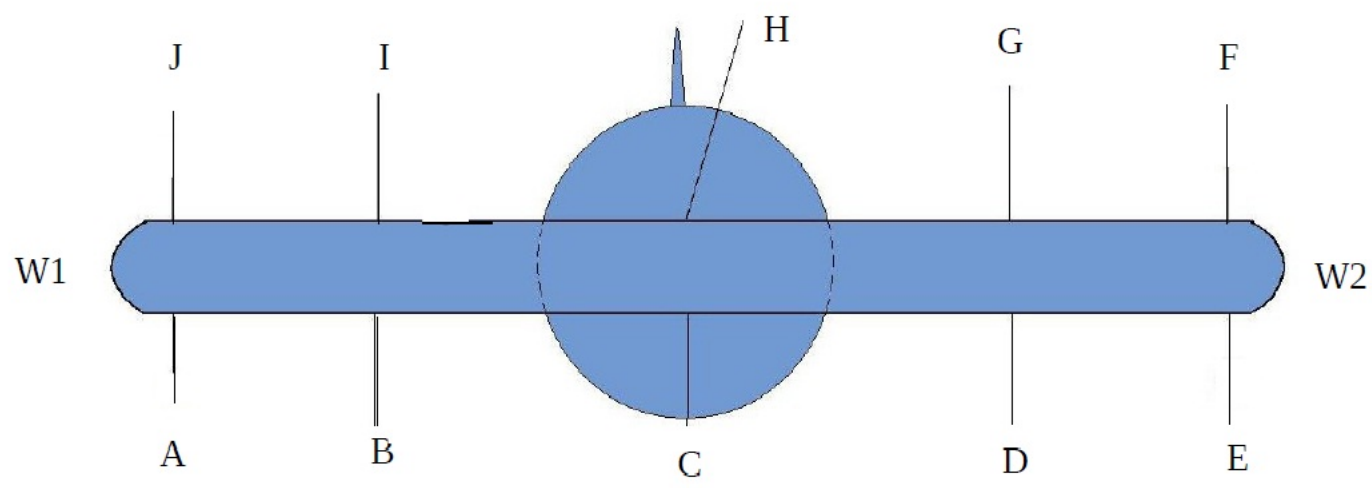

Figure 5.7: An example of measured positions in wall thickness measurements.

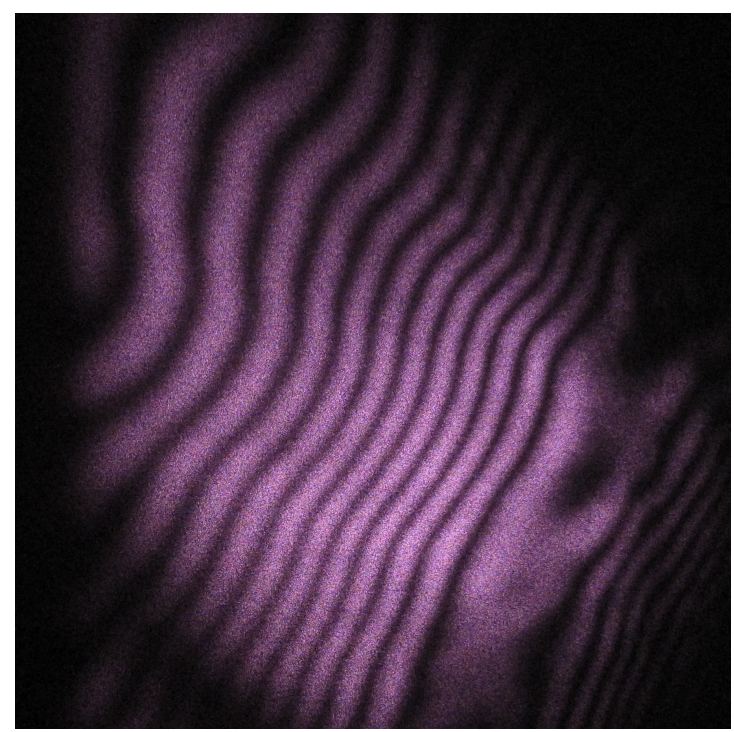

Figure 5.8: An example picture of the interference pattern. 
angle between the incident and the rejected light roughly around $20^{\circ}$. Since the angle is the main systematic uncertainty of this measurement, it is important to keep it under this limit to reduce its introduced error. A distinct interference spot can be found after several redundant movements of the cell, as shown in Figure 5.8. Thus, the iris is used to focus on either the largest dark or light stripes of the interference pattern. Once we are satisfied with the spot selection, the LabView program is launched to record and analyze the scanned data. It is important before starting a new data scan to record the position and angle information of the last measured point. This was done manually using a ruler and a protractor. The associated uncertainty with these manual measurements fluctuates respectively within the range of $0.75 \mathrm{~cm}$ and 2 degrees for the position and angle. Once the angle is recorded, Snell's law can be used to calculate the refraction angle. The intensity of light reflected from a flat surface is given by:

$$
I_{r}=\frac{\eta}{1-\eta} I_{0}
$$

where

$$
\eta=\frac{4\left(\frac{n-1}{n+1}\right)^{2}}{1-\left(\frac{n-1}{n+1}\right)^{2}} \sin ^{2}\left(\frac{2 \pi n d \cos \theta}{\lambda}\right)
$$

Here, $n$ is the index of refraction, $\lambda$ is the wavelength of the laser, $\theta$ is the angle of the reflection and $d$ is the thickness of the surface. The results of the wall thickness can be found in Appendix A.

\subsubsection{Lasers and Optics}

Three COMET lasers with a narrow wavelength line-width of $\approx 0.2 \mathrm{~nm}$ were used during the experiment, significantly improved the target polarization over previous experiments. With these three COMET lasers, a maximum polarization of $\approx$ $70 \%$ was achieved in the pumping chamber during the experiment. The currents 
Table 5.4: Laser parameters used throughout the experiment.

\begin{tabular}{|c|ccc|}
\hline Laser & Diode Current $(\mathrm{A})$ & Power $(\mathrm{W})$ & Diode Temperature $\left({ }^{\circ} \mathrm{C}\right)$ \\
\hline JLab1 & 35.0 & 25 & 26 \\
JLab2 & 34.0 & 25 & 20 \\
Rutgers & 35.9 & 25 & 25 \\
\hline
\end{tabular}

and the corresponding powers of the COMET lasers used in the experiment are listed in the Table 5.4. The lasers were installed and interlocked in the laser room behind the Counting House on the accelerator site at Jefferson Lab. Three 75-m long fibers guided the laser light from the laser room to the hall. One end of the fiber was connected to the output of the COMET laser, the other end was connected to a 5-to-1 combiner. A 5-to-1 combiner has five separate fibers as inputs and one output. During the entire period of production data taking, three $75 \mathrm{~m}$ fibers were connected to one 5 -to- 1 combiner with a typical power loss of $\approx$ $6 \%$ [52].

The laser light coming out of the 5-to-1 combiner was circularly polarized by passing through optical components. The setup of the optical components is shown in Figure 5.9. The laser light from the 5-to-1 combiner is divergent light with a wavelength of $795 \mathrm{~nm}$. A lens focusses the light to be parallel. A beam splitter was used to separate $\mathrm{S}$ and $\mathrm{P}$ waves from the incident unpolarized light coming out of the focusing lens. The $\mathrm{S}$ wave was allowed to pass through the quarter wave plate to reflect back from a mirror and pass through the quarter wave plate again. Therefore, after being reflected from the mirror and passing through the quarter wave plate twice, the $\mathrm{S}$ wave becomes a $\mathrm{P}$ wave and passes through the beam splitter again. On the other hand, the $\mathrm{P}$ wave light is incident on a second mirror and is reflected. Then both $\mathrm{P}$ waves pass through two quarter wave plates separately so that the linearly polarized $\mathrm{P}$ waves became circularly polarized. The quarter wave plates were calibrated to proper angles so that the 


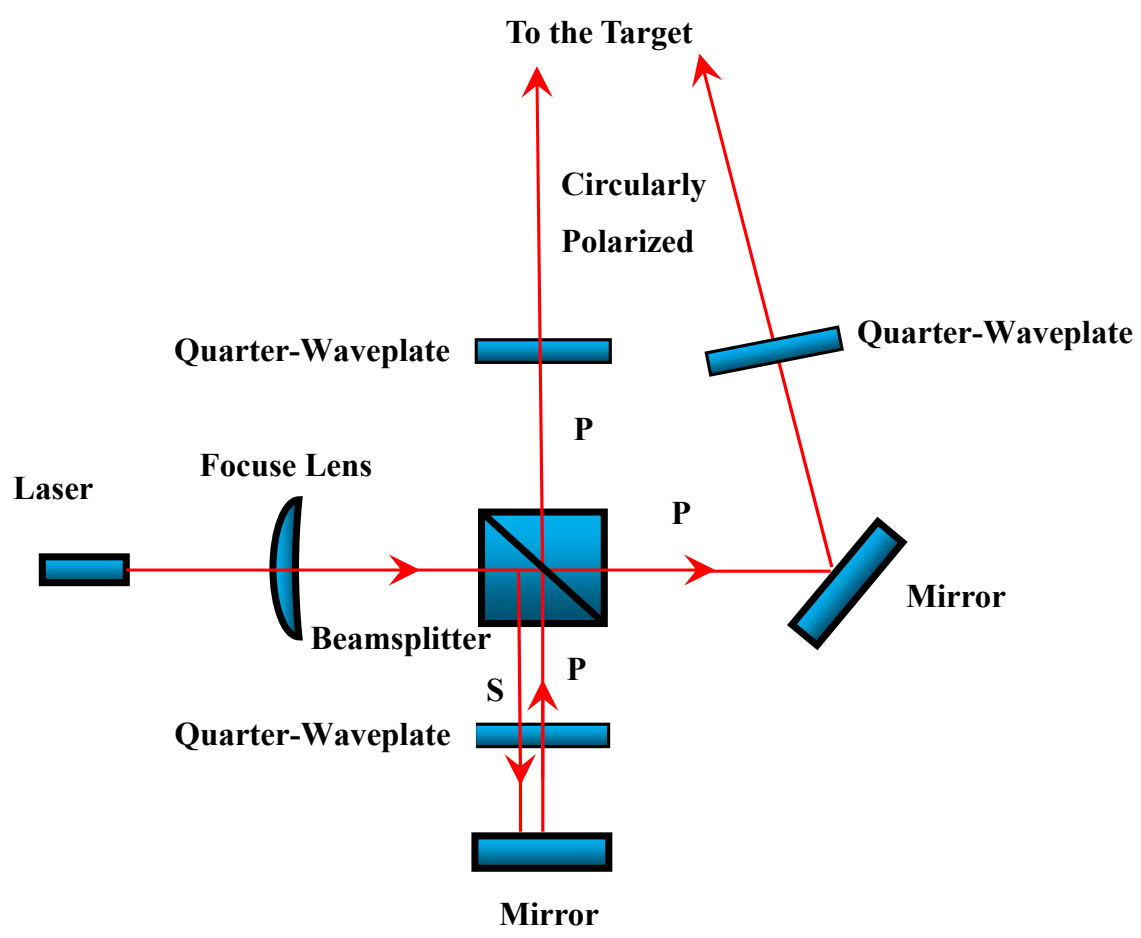

Figure 5.9: A schematic diagram of the optics setup.

linearly polarized $\mathrm{P}$ waves would be circularly polarized in the same direction (either left circularly polarized or right circularly polarized).

\subsubsection{Magnet System}

The polarized ${ }^{3} \mathrm{He}$ target system at Jefferson Lab has three pairs of Helmholtz coils: the Horizontal Small Coils, the Horizontal Large Coils and the Vertical Coils. The three coils are oriented in three mutually orthogonal directions as shown in Figure 5.10. They can provide a 3-dimensional magnetic field with an average strength of $25 \mathrm{G}$ and a typical gradient of $10-30 \mathrm{mG} / \mathrm{cm}$ in the region of the ${ }^{3} \mathrm{He}$ cell [52]. The magnitude and direction of the holding magnetic field were controlled by the electrical currents in these coils. The basic characteristics of these coils are shown in Table 5.5. The set current in each pair of coils that generated the vertical field is listed in Table 5.6. The direction of the holding field was measured to a precision of better than $0.5^{\circ}$ using a compass system. A 


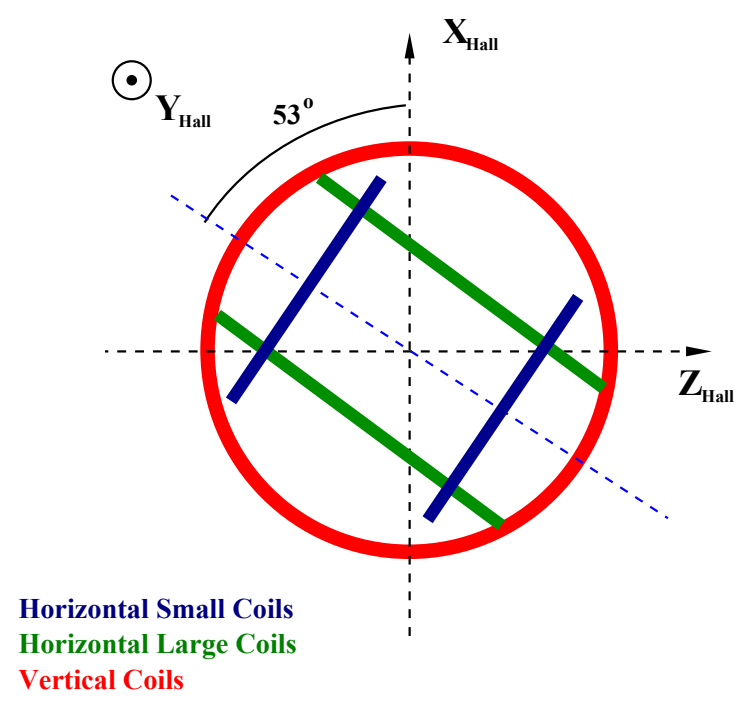

Figure 5.10: Top view of the coil setup for the ${ }^{3}$ He target.

Table 5.5: Basic characteristics and power supplies of Helmholtz coils.

\begin{tabular}{|c|ccc|}
\hline Coil Name & Hori. Small & Hori. Large & Vertical \\
\hline Inner Diameter $(\mathrm{m})$ & 1.27 & 1.45 & 1.83 \\
Number of Turns & 256 & 272 & 355 \\
Resistance $(\Omega)$ & 3 & 3 & 4.4 \\
\hline
\end{tabular}

detailed discussion about the compass measurements can be found in [67].

The RF coils provide an RF field with $f=91 \mathrm{kHz}$ and $B=100 \mathrm{mG}$, which were used to flip the ${ }^{3} \mathrm{He}$ spin.

\subsubsection{Oven, Heater, and Airflow System}

The pumping chamber was enclosed in an oven and kept at a high temperature in order to evaporate the alkali atoms in the ${ }^{3} \mathrm{He}$ cell. The oven used in the Table 5.6: Current set points for each coil for generating $25 \mathrm{G}$ holding field in vertical direction.

\begin{tabular}{|c|ccc|}
\hline Field & $I_{\text {Small }}(\mathrm{A})$ & $I_{\text {Large }}(\mathrm{A})$ & $I_{\text {Vertical }}(\mathrm{A})$ \\
\hline Vertical & 0.329 & -0.958 & 14.093 \\
\hline
\end{tabular}




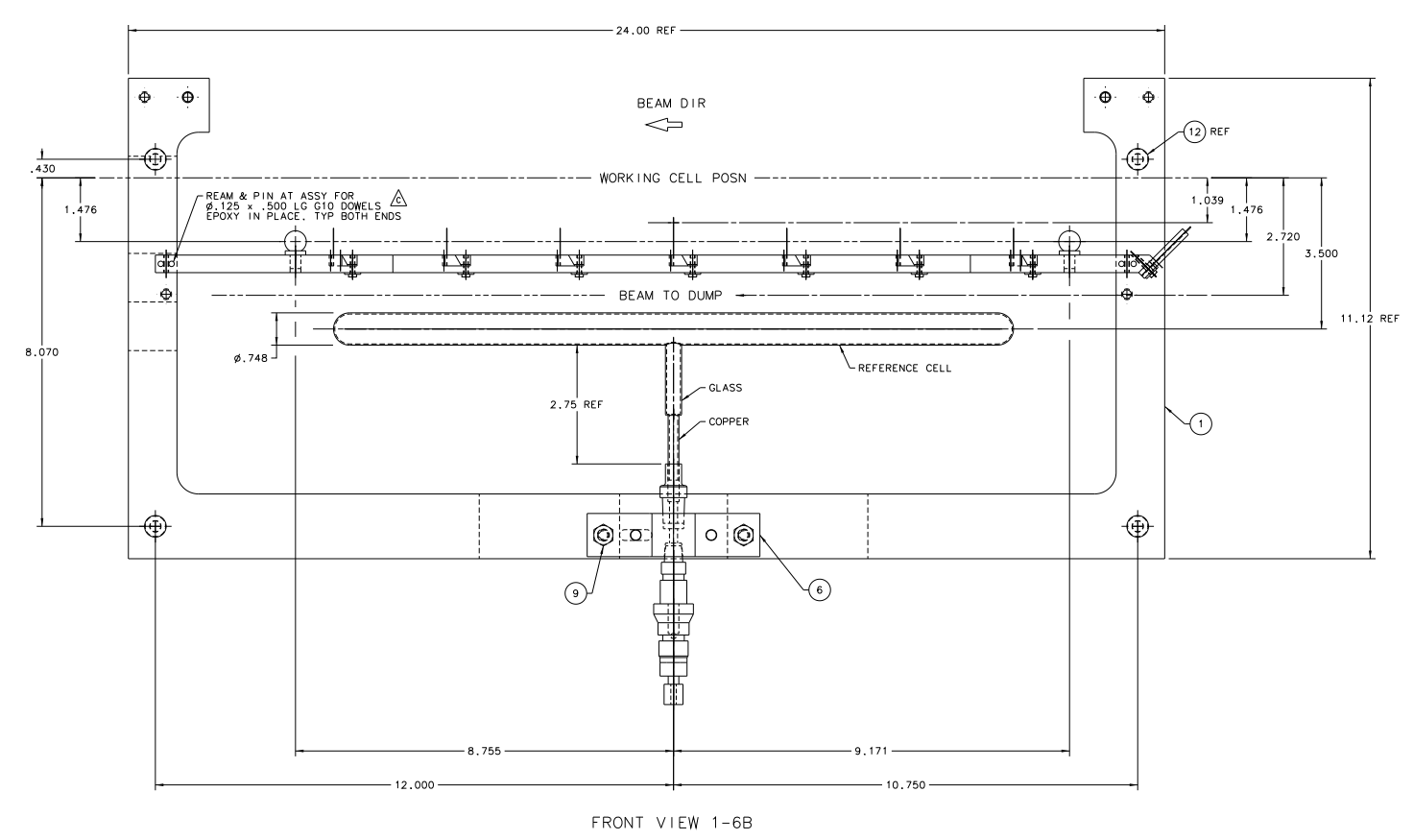

Figure 5.11: A front view of the target ladder.

experiment was made of a material called CS85 [52]. It had an inlet and an outlet for the circulation of compressed air. The air blown into the oven from the inlet was heated by two heaters. After circulating in the oven, the air exited from the outlet. Both the inlet and the outlet piping were enclosed in a tube that supported the oven and were wrapped with insulation. A RTD was attached inside the oven to read the temperature. Throughout the experiment, the oven temperature was kept stable at $230{ }^{\circ} \mathrm{C}$ with fluctuations within $2{ }^{\circ} \mathrm{C}$.

\subsubsection{Target Ladder}

During the experiment, a target ladder was mounted on the oven to allow five different target positions as shown in Figure 5.11. The ladder can move with the oven vertically to move the chosen target in the electron beam position. The five target positions are the following:

Polarized ${ }^{3}$ He target cell position: This position was for the 40-cm long polarized ${ }^{3} \mathrm{He}$ target chamber, which was used for the main production data 
in the experiment. The cell was glued to the oven bottom plate with RTV2.

A solid $\mathrm{BeO}$ foil with carbon foils target position: Seven carbon foils, equally spaced and covering the 40-cm length of the target chamber, were used for spectrometer optics calibration. The beryllium oxide (BeO) foil was used to make the beam spot visible to locate the center of the target.

A "hole" target position: This was the central carbon foil but extended in height with a hole in it. It was used for fine position alignment.

Empty target position: This position was mostly used for beam tuning.

Reference cell target position: This position was for different calibrations such as the elastic calibration, background studies, etc. The reference cell was filled with either nitrogen, hydrogen or helium-3 gas in accordance with the purpose of the studies.

\subsubsection{Cell Temperature and Density During E05-015}

Using the ideal gas law, the density of the target chamber is determined by:

$$
n_{t c}=n_{0}\left[1+\frac{V_{p c}}{V_{t o t}}\left(\frac{T_{t c}}{T_{p c}}-1\right)\right]^{-1},
$$

where $n_{0}$ is the density at room temperature, $V_{p c}$ is the internal volume of the pumping chamber, $V_{t o t}$ is the total internal volume of the cell, $T_{t c}$ is the average target chamber temperature and $T_{p c}$ is the average temperature of the pumping chamber. Similarly, the density of the pumping chamber is determined by:

$$
n_{p c}=n_{0}\left[1+\frac{V_{t c}}{V_{t o t}}\left(\frac{T_{p c}}{T_{t c}}-1\right)\right]^{-1}
$$

where $V_{t c}$ is the internal volume of the target chamber.

\footnotetext{
${ }^{2}$ Room Temperature Vulcanizing silicone.
} 


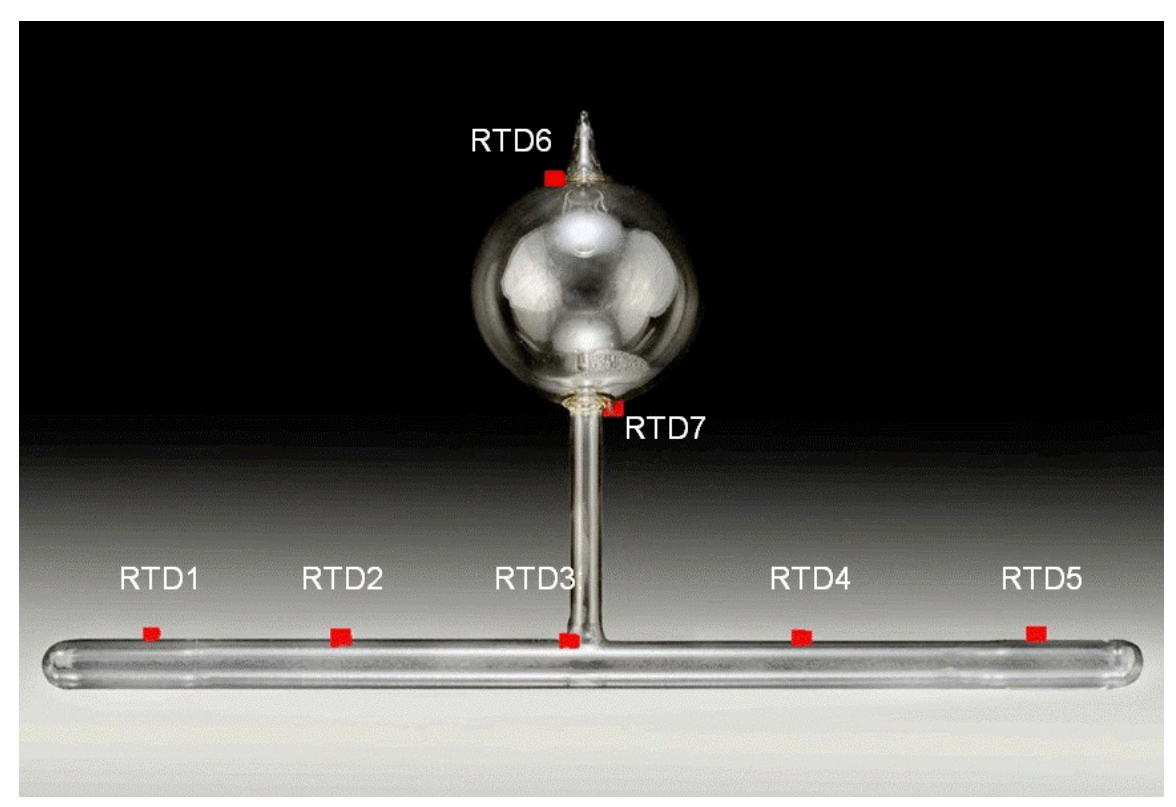

Figure 5.12: RTD positions on the ${ }^{3} \mathrm{He}$ target. RTD 1-5 are evenly positioned on the target chamber and RTD 6-7 are on the pumping chamber.

The cell temperature was measured with seven RTDs with their locations shown in Figure 5.12 , The target chamber temperature, $T_{t c}$, was calculated by simply taking an average of RTD 1-5. The pumping chamber also has two RTDs on it. However, these two RTDs only reflect the interior temperature of the cell when the lasers are off. When the lasers are on, there is a thermal gradient between the inner part of the cell and the edge of the pumping chamber. In order to measure the average internal pumping chamber temperature when the lasers are on, a separate measurement using the NMR system (Section 5.3) was performed. This measurement compares the NMR signal height when the lasers are on and when the lasers are off. More details about the temperature test can be found in [68]. Analysis of the measurements found that the difference between the RTD reading and the internal temperature of the pumping chamber was $\approx 16$ ${ }^{\circ} \mathrm{C}$. Using the temperature test results, the interpolated temperature to each ${ }^{3} \mathrm{He}$ run taken during the experiment is illustrated in Figure 5.13, and the interpolated density to each ${ }^{3}$ He run is illustrated in Figure 5.14 . 


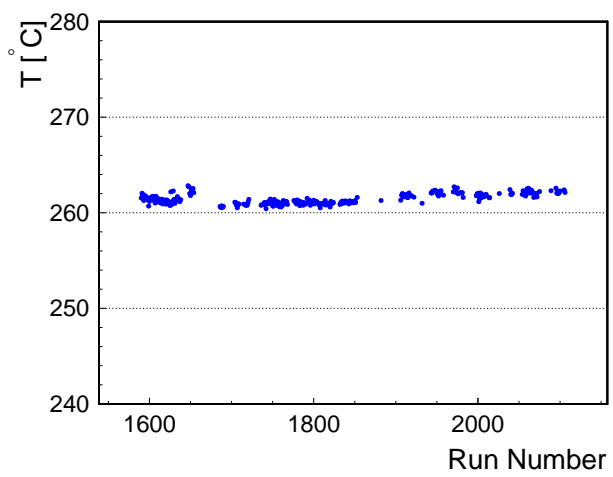

(a)

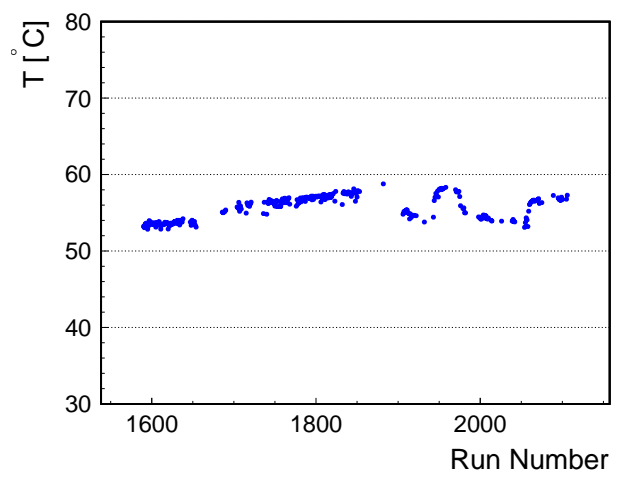

(b)

Figure 5.13: Temperature in the cell during E05-015. (a) Temperature in the pumping chamber. (b) Temperature in the target chamber.

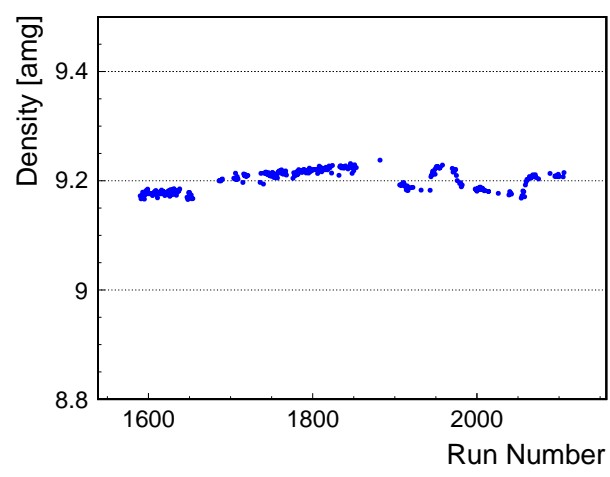

(a)

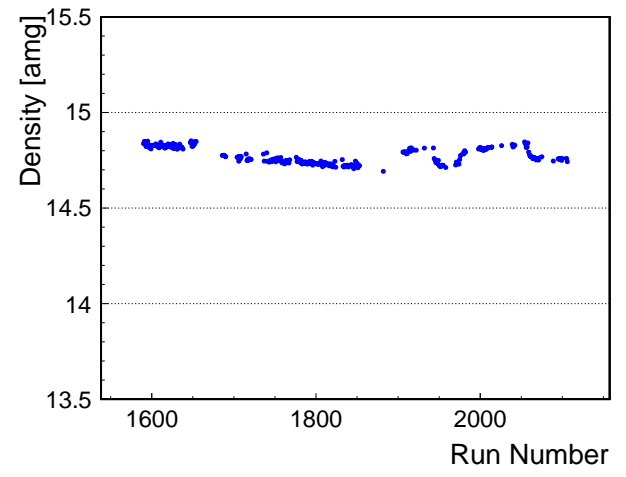

(b)

Figure 5.14: Density in the cell during E05-015. (a) Density in the pumping chamber. (b) Density in the target chamber. 


\subsection{Nuclear Magnetic Resonance (NMR) Polarimetry}

NMR polarimetry determines the target polarization by measuring the ${ }^{3} \mathrm{He}$ NMR signal during spin reversal of the ${ }^{3} \mathrm{He}$ nuclei through the adiabatic fast passage (AFP) [69] technique. During the experiment, the spins of the ${ }^{3} \mathrm{He}$ nuclei were reversed every 20 minutes, and an NMR measurement was recorded during each flip. Therefore, the polarization was determined and recorded every 20 minutes.

\subsubsection{AFP Process}

When a particle with spin $\vec{I}$ is put into a uniform magnetic field $\vec{B}_{0}$, the particle will rotate around $\vec{B}_{0}$. The rotation equations are as follows:

$$
\begin{gathered}
\vec{M}=\gamma \vec{I} \\
\vec{L}=\vec{M} \times \vec{B}_{0} \\
\frac{d \vec{I}}{d t}=\vec{L},
\end{gathered}
$$

where $\vec{M}$ is the magnetic moment, $\gamma$ is the gyro-magnetic ratio and $\vec{L}$ is the angular momentum. Combine the equations yields

$$
\frac{d \vec{M}}{d t}=\gamma\left(\vec{M} \times \vec{B}_{0}\right)
$$

Adding an additional small field $\vec{B}_{1}$, which is perpendicular to $\vec{B}_{0}$ and rotates around with an angular frequency $\omega$ in the opposite direction to $\vec{B}_{0}$, changes Equation 5.10 to

$$
\frac{d \vec{M}}{d t}=\gamma \vec{M} \times\left(\vec{B}_{0}+\vec{B}_{1}\right)
$$

Now this equation is in the laboratory frame of reference. To simplify, let us consider a rotating frame of reference, S', rotating with an angular velocity $-\vec{\omega}$ 
with respect to the laboratory frame. In $\mathrm{S}^{\prime}$ frame, $\vec{B}_{0}=B_{0} \hat{y},-\vec{\omega}=-\omega \hat{y}$ and $\vec{B}_{1}=B_{1} \hat{z}$, and we can write the motion of the magnetic moment in the rotating frame S' as:

$$
\begin{aligned}
\frac{d \vec{M}}{d t} & =\gamma \vec{M} \times\left(\vec{B}_{0}-\frac{\vec{\omega}}{\gamma}+\overrightarrow{B_{1}}\right) \\
& =\gamma \vec{M} \times\left[\left(B_{0}-\frac{\omega}{\gamma}\right) \hat{y}+B_{1} \hat{z}\right]
\end{aligned}
$$

Now if we compare Equation (5.11) and Equation (5.13), an effective field $B_{e}$ can be given by

$$
\vec{B}_{e}=\left(B_{0}-\frac{\omega}{\gamma}\right) \hat{y}+B_{1} \hat{x}
$$

The target spin $\vec{S}$ is primarily oriented parallel to the holding field $\vec{B}_{0}$. During the AFP process, the magnetic field $\left(B_{0}-\frac{\omega}{\gamma}\right)$ is slowly changed from $\left(B_{0}-\frac{\omega}{\gamma}\right)<<$ $-B_{1}$, passes zero and ends at $\left(B_{0}-\frac{\omega}{\gamma}\right)>>B_{1}$. If the change is slow enough, the target spin will follow the direction of $\vec{B}_{e}$ and reverse its direction.

\subsubsection{NMR Measurement}

During the experiment, the rotation field $B_{1}$ was generated by RF coils. This field was perpendicular to $\vec{B}_{0}$ with an angular frequency of $|\omega|$. There are two types of AFP techniques used to introduce a time dependent $\left(B_{0}-\frac{\omega}{\gamma}\right)$. Both were used in E05-015.

AFP field sweep: If the holding field is swept and the frequency is kept constant, the usual AFP condition requires a sweep rate of $1.2 \mathrm{G} / \mathrm{s}$. In this case, the holding field is swept from $25 \mathrm{G}$ to $32 \mathrm{G}$ and then back with the resonance at $28 \mathrm{G}$, which corresponds to a transition frequency of $91 \mathrm{kHz}$. During the experiment, field sweep NMR was done a few times as part of our calibration process. This allowed us to compare the pumping chamber polarization with the target chamber polarization. 


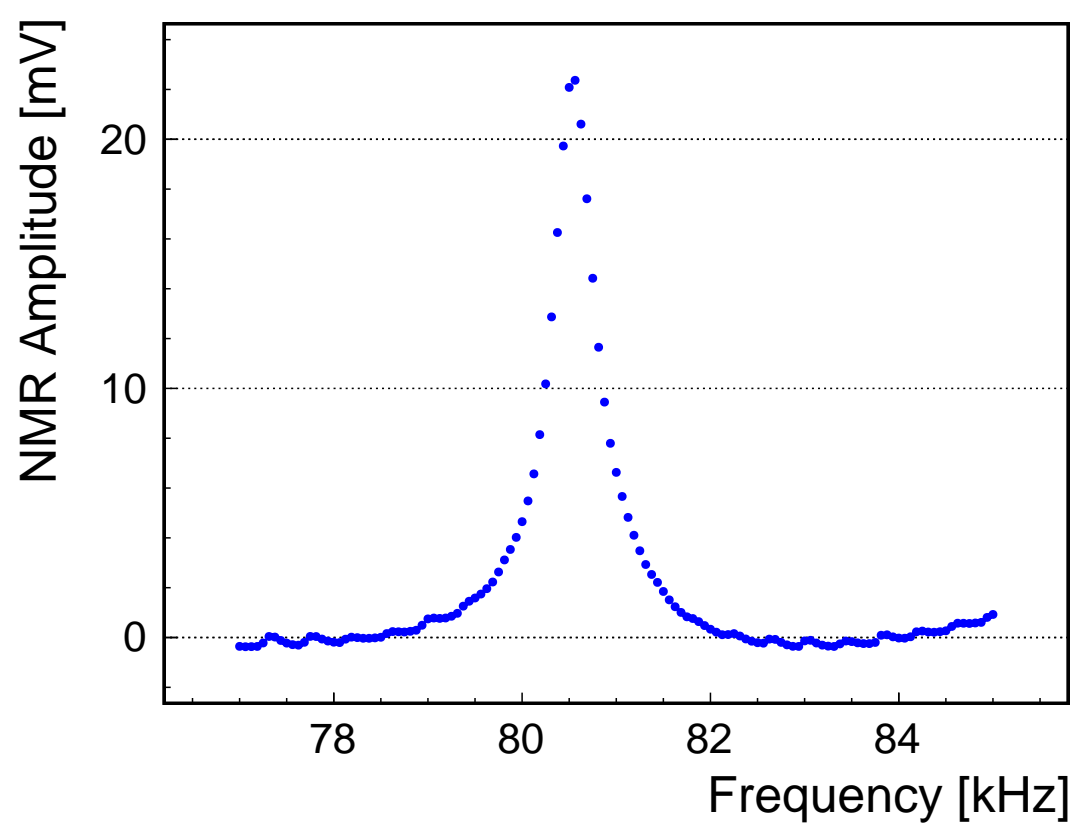

Figure 5.15: A typical NMR frequency sweep signal.

AFP frequency sweep: For frequency sweep, the holding field is kept constant and the RF was swept from $77 \mathrm{kHz}$ to $85 \mathrm{kHz}$ through the resonance at $\omega_{0}=81 \mathrm{kHz}$ and back. The sweep rate was $4 \mathrm{kHz} / \mathrm{s}$ to satisfy the AFP conditions. During the production running, only frequency sweep measurements were performed due to their lower signal to noise ratio.

A typical NMR signal using the frequency sweep method is shown in the Figure 5.15. The height of the signal from the lock-in amplifier is proportional to the ${ }^{3}$ He polarization. The function used to fit the signals is:

$$
S_{\mathrm{NMR}}=A \cdot \frac{B_{1}}{\sqrt{\left(B_{0}-\frac{\omega}{\gamma}\right)^{2}+B_{1}^{2}}}+\text { Background }
$$

where $S$ is the signal in the pick-up coils and $A$ is the amplifier. 


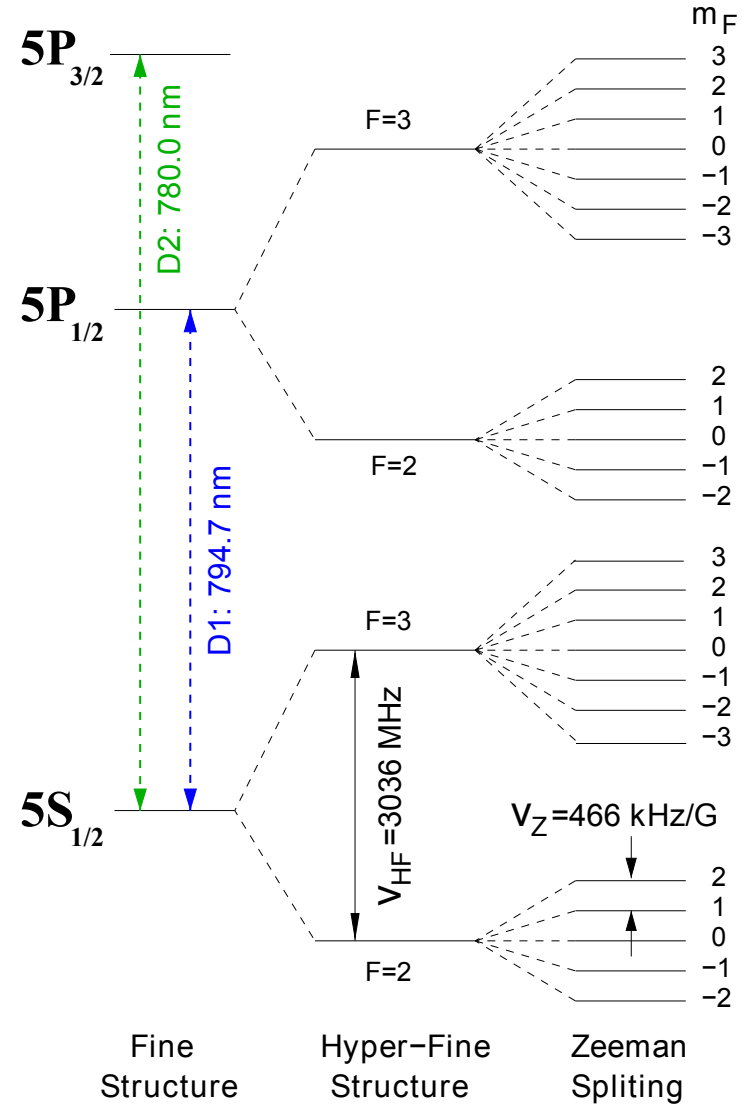

Figure 5.16: Energy level of ${ }^{85} \mathrm{Rb}$ [67]. The Zeeman splitting is given by $\nu_{Z}$. 


\subsection{Electron Paramagnetic Resonance (EPR) Polarimetry}

The method of Electron Paramagnetic Resonance (EPR) measures the absolute polarization of the ${ }^{3} \mathrm{He}$ nuclei in the pumping chamber. Besides the magnetic field generated by the magnetic coils, polarized ${ }^{3} \mathrm{He}$ can generate a weak magnetic field. This weak magnetic field can shift the frequency of the rubidium or potassium Zeeman resonance. The polarization of ${ }^{3} \mathrm{He}$ can be determined by measuring the resonance frequency shift, i.e.,

$$
\nu_{\mathrm{EPR}}=\nu_{0} \pm \Delta \nu_{\mathrm{EPR}}
$$

where $\nu_{0} \propto B_{0}, \Delta \nu_{\mathrm{EPR}} \propto P_{\mathrm{He}}$.

The first step in understanding electron paramagnetic resonance is to understand the level splitting of the $\mathrm{Rb}$. Rb has a single electron in the outer shell $\left(5 S_{1 / 2}\right)$, and its interaction Hamiltonian with a magnetic field $\vec{B}$ is given by [15]:

$$
\hat{H}=A_{g} \vec{I} \cdot \vec{S}+g_{e} \mu_{B} S_{y} B_{y}-\frac{\mu_{I}}{I} I_{y} B_{y},
$$

where the first term is the vector compling between the electron spin $\vec{S}$ and the nuclear spin $\vec{I}$, the second term is the coupling of the electron spin to the magnetic field $B_{y}$, and the third term is the coupling of the nuclear spin to the magnetic field $B_{y}$. Solutions of Equation (5.17) give the eigenstates of Rb atoms, which are labeled by the quantum number $F=I \pm S$. In this case, $F=2$ and $F=3$. In a magnetic field, a state $F$ splits into $2 F+1$ sublevels labeled by $m_{F}$. For $F=2, m_{F}=-2,-1,0,1,2$ and for $F=3, m_{F}=-3,-2,-1,0,1,2$, 3 as shown in Figure 5.16. The Zeeman splitting between $\left(F=3, m_{F}=-3\right)$ and $\left(F=3, m_{F}=-2\right)$ or between $\left(F=3, m_{F}=3\right)$ and $\left(F=3, m_{F}=2\right)$ is described by the Electron-Paramagnetic Resonance frequency $\nu_{\mathrm{EPR}}$, which is proportional to the magnetic field $\vec{B}$. In order to cancel the large component $\nu_{0}$, which is generated by the main holding field, the ${ }^{3} \mathrm{He}$ spins are reversed by AFP and the frequency shift $\Delta \nu_{\mathrm{EPR}}$ was measured directly during an EPR measurement. By flipping 
Table 5.7: Parameters used in extracting polarization from EPR signals.

\begin{tabular}{|c|ccc|}
\hline Parameters & Description & Value & Unit \\
\hline $2 \Delta \nu$ & Frequency difference & From the experiment & $\mathrm{Hz}$ \\
$\mu_{0}$ & Vacuum permeability & $4 \pi \times 10^{-7}$ & $\mathrm{~N} / \mathrm{A}^{2}$ \\
$\frac{d \nu_{\mathrm{EPR}}}{d B}$ & Constant from atomic physics experiments & See the content & $\mathrm{Hz} / \mathrm{T}$ \\
$\kappa_{0}$ & Constant from atomic physics experiments & See the content & $\mathrm{T}$ is in ${ }^{\circ} \mathrm{C}$ \\
$\mu^{3} \mathrm{He}$ & Magnetic moment of ${ }^{3} \mathrm{He}$ & $1.075 \times 10^{-26}$ & $\mathrm{~J} / \mathrm{T}$ \\
$n_{p c}$ & ${ }^{3} \mathrm{He}$ density in pumping chamber & From the experiment & Amagat \\
\hline
\end{tabular}

the ${ }^{3} \mathrm{He}$ spin with AFP frequency sweep, the frequency shifts $\left(2 \Delta \nu_{\mathrm{EPR}}\right)$ can be measured to a precision of $\delta\left(\Delta \nu_{\mathrm{EPR}}\right) /\left(\Delta \nu_{\mathrm{EPR}}\right) \approx 0.2 \%$.

The transition frequency between the Rb sublevels is proportional to the external magnetic field, which is in our case defined as $B_{0}+\Delta B_{3^{3} \mathrm{He}}$. Using an RF coil, the resonance frequency can be found for the external field $B_{0}+\Delta B_{{ }^{3} \mathrm{He}}$, and then, by flipping the ${ }^{3} \mathrm{He}$ spins, one can access the resonance frequency for the other external field $B_{0}-\Delta B_{3^{3} \mathrm{He}}$. Therefore the target's polarization is proportional to the resonance frequency shift $(2 \Delta \nu)$ :

$$
P_{{ }^{3} \mathrm{He}}=\frac{1}{\kappa_{\mathrm{EPR}}} \Delta \nu,
$$

where $\kappa_{\mathrm{EPR}}$ is:

$$
\kappa_{\mathrm{EPR}}=\frac{2 \mu_{0}}{3} \frac{d \nu_{\mathrm{EPR}}}{d B} \kappa_{0} \mu_{3 \mathrm{He}} n_{\mathrm{pc}} .
$$

Substituting Equation (5.19) into Equation (5.18) yields:

$$
\Delta \nu=\frac{2 \mu_{0}}{3} \frac{d \nu_{\mathrm{EPR}}}{d B} \kappa_{0} \mu_{3 \mathrm{He}} n_{\mathrm{pc}} P_{3 \mathrm{He}},
$$

where the individual parameters are listed in Table 5.7.

A recent measurement of $\kappa_{0}$ was carried out by Babcock, et al. [70. In their measurement, $\kappa_{0}(T)$ is reported as two parts: a static value $\left(\kappa_{0}\right)$ and a temperature dependent piece $\left(\kappa_{0}^{\prime}\right)$ with:

$$
\kappa_{0}(T)=\kappa_{0}\left(T_{\text {ref }}\right)+\kappa_{0}^{\prime}\left(T-T_{\text {ref }}\right),
$$


where $T_{\text {ref }}$ is a given reference temperature. For $\mathrm{Rb}$ and $\mathrm{K}$ at $T_{\text {ref }}=200^{\circ} \mathrm{C}$, the $\kappa_{0}$ are:

$$
\kappa_{0}^{R b}(T)=6.39+0.00914(T-200)
$$

and

$$
\kappa_{0}^{K}(T)=(5.99 \pm 0.11)+(0.0086 \pm 0.0020)(T-200)
$$

The target pumping chamber temperature during the experiments is approximately $270{ }^{\circ} \mathrm{C}$. At these high temperatures, the uncertainty on $\kappa_{0}^{K}$ due to the temperature dependence is $2.3 \%$. When the systematic uncertainty on the reference value is combined, the total systematic uncertainty on $\kappa_{0}^{K}$ is $3.0 \%$.

To fifth order in magnetic field, the derivative of the frequency with respect to the field is given by [50]:

$$
\frac{d \nu_{ \pm}}{d B}=\frac{g_{I} \mu_{N}-g_{S} \mu_{B}}{h[I]} \sum_{n=0}^{5} b_{n} \frac{x^{n}}{[I]^{n}},
$$

where

$$
\begin{gathered}
x=\left(g_{I} \mu_{N}-g_{S} \mu_{B}\right) \frac{B}{h \nu_{h f s}} \\
{[I]=2 I+1} \\
b_{0}=1 \\
b_{1}=\mp 4 I \\
b_{2}=6 I(2 I-1) \\
b_{3}=\mp 8 I\left(4 I^{2}-6 I+1\right) \\
b_{4}=10 I(2 I-1)\left(4 I^{2}-10 I+1\right),
\end{gathered}
$$

and

$$
b_{5}=\mp 12 I\left(16 I^{4}-80 I^{3}+80 I^{2}-20 I+1\right) \text {. }
$$

The individual parameters used in the calculation are listed in Table 5.8 . 
Table 5.8: Parameters used in $\frac{d \nu_{\mathrm{EPR}}}{d B}$ calculation.

\begin{tabular}{|c|ccc|}
\hline Parameters & Description & Value & Unit \\
\hline$g_{I}$ & K g-factor & 0.2601 & - \\
& Rb g-factor & 0.5412 & - \\
$\mu_{N}$ & nucler magneton & $5.051 \times 10^{-27}$ & $\mathrm{~J} / \mathrm{T}$ \\
$g_{S}$ & electron g-factor & 2.0023 & - \\
$\mu_{B}$ & Bohr magneton & $9.275 \times 10^{-24}$ & $\mathrm{~J} / \mathrm{T}$ \\
$h$ & Plank's constant & $6.626 \times 10^{-34}$ & $\mathrm{Js}$ \\
$I$ & K nuclear spin & 1.5 & $\hbar$ \\
& Rb nuclear spin & 2.5 & $\hbar$ \\
$\nu_{h f s}$ & K & $461.719 \times 10^{6}$ & $\mathrm{~Hz}$ \\
& Rb & $3035.732 \times 10^{6}$ & $\mathrm{~Hz}$ \\
\hline
\end{tabular}

\subsection{Polarization Results from EPR Measurement}

A typical EPR online measurement is shown in Figure 5.17. During E05-015, five EPR calibration measurements were done. Using Equation (5.20), the polarization results were extracted as shown in Figure 5.18 .

\subsection{Calibration of NMR with EPR}

For each AFP sweep during the EPR measurements, an NMR measurement was performed using the identical settings as the AFP spin flips for production running as shown in Figure 5.17. The NMR calibration constant, $C$, can be extracted as

$$
C_{i}=P_{\mathrm{EPR}, i} / S_{\mathrm{NMR}, i}
$$

where $P_{\mathrm{EPR}, i}$ is the EPR polarization evaluated for the $i^{\text {th }}$ flip and $S_{\mathrm{NMR}, i}$ is the NMR signal height. This value is $2.39 \pm 0.05 \% / \mathrm{mV}$ for this experiment.

\subsection{Polarization Gradients}

As is described in Section 5.2.1, the ${ }^{3} \mathrm{He}$ target cell is divided into three parts: the pumping chamber, the transfer tube and the target chamber. During this 


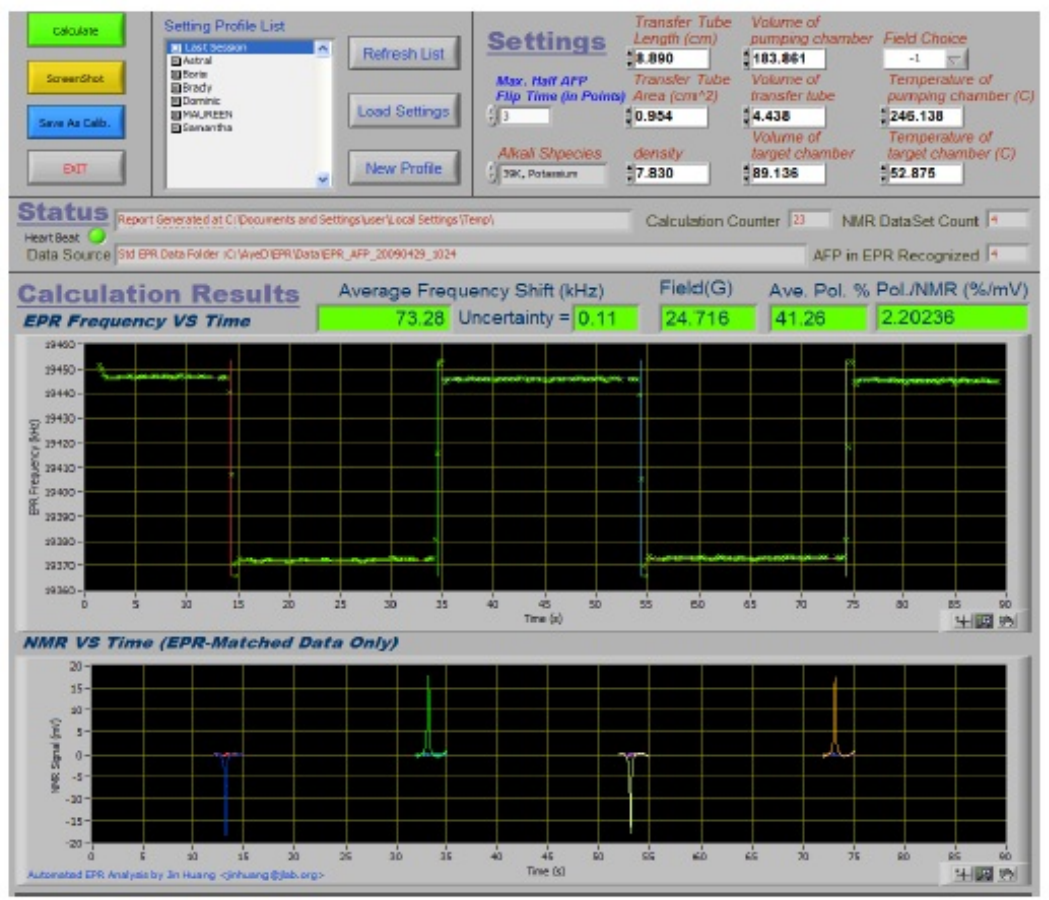

Figure 5.17: An example of an online EPR measurement.

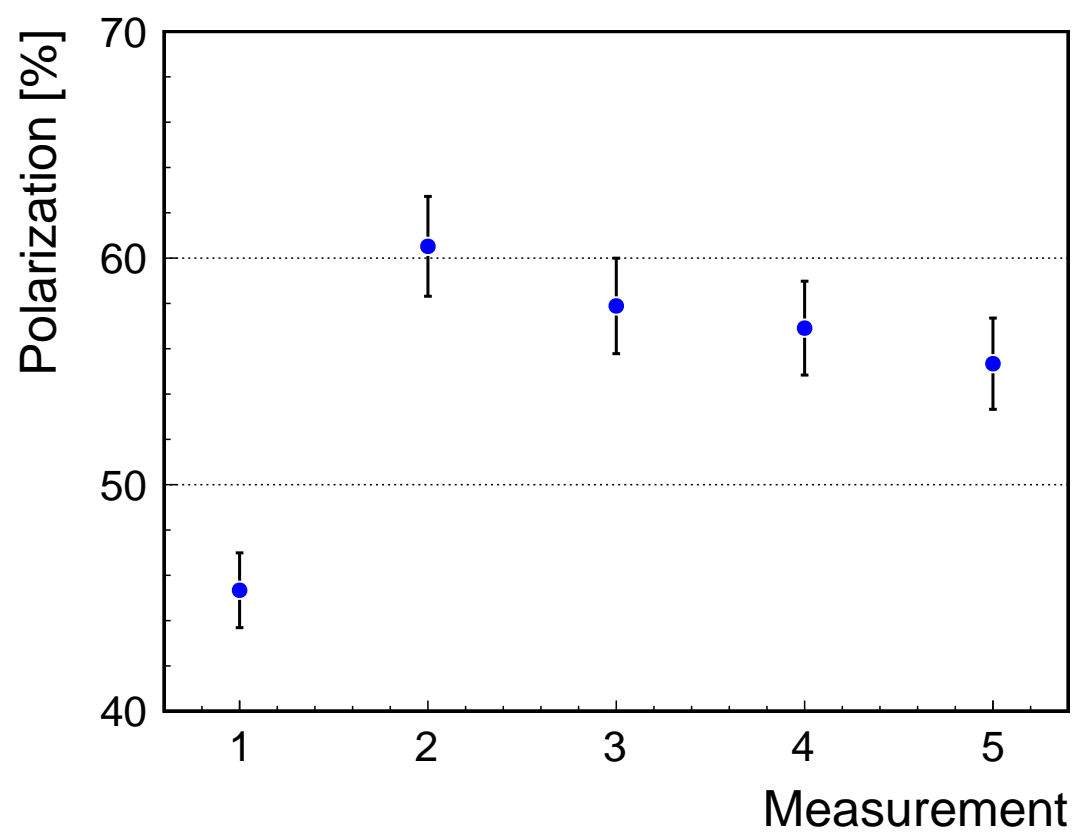

Figure 5.18: EPR polarization results from the calibration measurements. 
experiment, NMR polarimetry, calibrated by EPR measurements, measured the pumping chamber polarization, $P_{p c}$, every 20 minutes. However, the electron interacted with the polarized ${ }^{3} \mathrm{He}$ gas in the target chamber, the polarization of which is $P_{t c}$. Experiment design requirements did not allow pick up coils around the target chamber, so the polarization in the target chamber could not be directly monitored. ${ }^{3}$ He gas was polarized in the pumping chamber, and diffused to the target chamber by the transfer tube, so the polarization in the pumping chamber and the target chamber are different. The polarization gradient between the two chambers is quantified as $P_{t c} / P_{p c}$. In order to obtain the polarization in the target chamber, the polarization in the pumping chamber must be corrected for this polarization gradient. The gradient will be discussed in the next section using a two-chamber polarization model.

\subsubsection{Two Chamber Polarization Model}

The polarization dynamics can be described by a two-chamber model [71] [72]:

$$
\begin{gathered}
\frac{d P_{p c}}{d t}=d_{p c}\left(P_{t c}-P_{p c}\right)+\gamma_{S E}\left(P^{A}-P_{p c}\right)-\Gamma_{p c} P_{p c} \\
\frac{d P_{t c}}{d t}=d_{t c}\left(P_{p c}-P_{t c}\right)-\Gamma_{t c} P_{t c}
\end{gathered}
$$

where $P^{A}, P_{p c}$ and $P_{t c}$ are the polarizations of alkali, pumping chamber and target chamber, respectively, $\gamma_{S E}$ is the spin-exchange rate in the pumping chamber, $\Gamma_{p c}$ and $\Gamma_{t c}$ are the ${ }^{3} \mathrm{He}$ spin relaxation rates in each chamber due to effects other than spin-exchange and diffusion.

During E05-015, almost all data were taken near the maximum ${ }^{3} \mathrm{He}$ polarization. When the ${ }^{3} \mathrm{He}$ target is pumped for a long enough time, the polarization in the pumping chamber and target chamber reaches an equilibrium state, the polarization inside the two chambers does not change with time, and the left side of Equation (5.34) and Equation (5.35) are zero. The solution to Equation (5.34) 
and Equation 5.35) can be written as [52]:

$$
\begin{gathered}
P_{p c}^{\infty}=P^{A} \frac{\gamma_{S E} f_{p c}}{\gamma_{S E} f_{p c}+\Gamma_{p c} f_{p c}+\frac{\Gamma_{t c} f_{t c} d_{t c}}{d_{t c}+\Gamma_{t c}}} \\
P_{t c}^{\infty}=P_{p c}^{\infty} \frac{d_{t c}}{d_{t c}+\Gamma_{t c}},
\end{gathered}
$$

where $f_{p c}\left(f_{t c}\right)$ is the fraction of ${ }^{3}$ He nuclei in the pumping (target) chamber. From Equation (5.37), one can see that the polarization gradient $P_{t c} / P_{p c}$, is determined by two parameters, $d_{t c}$ and $\Gamma_{t c}$.

\subsubsection{Diffusion Rate $d_{t c}$}

From previous work [15], the diffusion rate $d_{t c}$ can be written as:

$$
\begin{gathered}
d_{t c}=\frac{A_{t t}}{V_{t c} L_{t t}} D_{t c} K \\
K=\frac{(2-m)(t-1)}{t^{2-m}-1} \\
t=\frac{T_{p c}}{T_{t c}}
\end{gathered}
$$

where $A_{t t}$ is the cross-sectional area of the transfer tube, $L_{t t}$ is the length of the transfer tube, and $V_{t c}$ is the internal volume of the target chamber. $D_{t c}$ is the diffusion constant, which can be described with a model based on a classical gas of hard spheres 71

$$
D_{t c}=D_{0}\left(\frac{T_{t c}}{T_{0}}\right)^{m-1} \frac{n_{0}}{n_{t c}}
$$

where $D_{0}=2.789 \pm 0.007 \mathrm{~cm}^{2} / \mathrm{s}$ is the diffusion constant of ${ }^{3} \mathrm{He}$ gas at $T_{0}=353$ $\mathrm{K}$ and $\mathrm{m}=1.705 \pm 0.003$ describes the temperature dependence of the diffusion constant. For E05-015, the typical value of $d_{t c}$ is $(1.8 \text { hour })^{-1}$, with an uncertainty estimated as $\delta d_{t c} / d_{t c} \approx 17 \%$. 


\subsubsection{Target Chamber Life Time}

Spin relaxation in the target chamber arises from several processes [52]:

$$
\Gamma_{t c}=\Gamma_{h e}+\Gamma_{\text {wall }}+\Gamma_{\text {beam }}+\Gamma_{A F P}+\Gamma_{\Delta B} .
$$

The various terms are the following:

$\Gamma_{h e}$ (Nuclear dipolar interaction): the depolarizing interactions due to the coupling between two adjacent ${ }^{3} \mathrm{He}$ nuclei. This number was calculated by Newbury, et. al. [73] as

$$
\Gamma_{h e}=\frac{n}{(744 \text { amagat } \cdot \text { hour })}
$$

at $23{ }^{\circ} \mathrm{C}$, where $n$ is the density of ${ }^{3} \mathrm{He}$. Based on Newbury's study, an empirical formulas including the temperature dependence was proposed by J. Singh [71] to calculate $\Gamma_{h e}$ as:

$$
\Gamma_{h e}=\frac{n}{(744 \text { amagat } \cdot \text { hour }) \cdot f(T)},
$$

where $f(T)$ is a function of temperature:

$$
f(T)=c_{0} \cdot\left(\frac{T}{T_{0}}\right)^{c_{1}}+c_{2}+c_{3} \cdot\left(\frac{T}{T_{0}}\right)+\frac{c_{4}}{1+c_{5} \cdot \frac{T}{T_{0}}} .
$$

The parameters can be found in Table 5.9. When $\mathrm{T}=\mathrm{T}_{0}=23{ }^{\circ} \mathrm{C}, \mathrm{f}(\mathrm{T})=1$.

$\Gamma_{\text {wall }}$ (Wall relaxation): the depolarization effect due to collisions between the ${ }^{3} \mathrm{He}$ nucleus and the cell glass wall. The average wall relaxation for both chambers can be written as:

$$
\Gamma_{\text {wall }}=\frac{1}{\tau_{\text {lifetime }}^{\text {cold }}}-\Gamma_{\text {he }}^{\text {cold }}
$$

where $\tau_{\text {lifetime }}^{\text {cold }}$ is the life time with the target cell at room temperature and $\tau_{h e}^{\text {cold }}$ is the ${ }^{3}$ He nuclear dipolar interaction at room temperature. The 
Table 5.9: The constants used in Equation 5.45).

\begin{tabular}{|c|c|}
\hline parameter & value \\
\hline$c_{0}$ & $1.2319 \mathrm{E}+0$ \\
$c_{1}$ & $2.8591 \mathrm{E}-1$ \\
$c_{2}$ & $-2.1793 \mathrm{E}-1$ \\
$c_{3}$ & $-1.4426 \mathrm{E}-2$ \\
$c_{4}$ & $5.3315 \mathrm{E}-1$ \\
$c_{5}$ & $1.2376 \mathrm{E}+3$ \\
$T_{0}$ & $296.15 \mathrm{~K}$ \\
\hline
\end{tabular}

life time was measured after the experiment in the target lab as shown in Figure 5.19. On the other hand, if one assumes the glass relaxation in both chambers is the same, $\Gamma_{\text {wall }}$ for both chambers will be proportional to their surface area to volume ratio $\mathrm{S} / \mathrm{V}$ [52]. The difference between $\Gamma_{\text {wall }}$ and $\Gamma_{\text {wall }, S / V}$ was used as the uncertainty of $\Gamma_{\text {wall }}$ and was estimated as $40 \%$.

$\Gamma_{\text {beam }}($ Beam depolarization): Ionization increases the nuclear spin relaxation in the target chamber as shown in Figure 5.20. The beam depolarization effect was extracted with

$$
\frac{{ }_{o f f} P_{p c}^{\infty}}{{ }_{o n} P_{p c}^{\infty}} \approx 1+\Gamma^{b e a m} \cdot \frac{n_{t c}}{n_{p c}} \cdot \tau,
$$

where ${ }_{o f f} P_{p c}^{\infty}\left({ }_{o n} P_{p c}^{\infty}\right)$ is the polarization in the pumping chamber after equilibrium is reached in the pumping chamber and target chamber when beam off (on). $\tau$ is the spin up time with the beam off, which was measured during the experiment as shown in Figure 5.21. It was found that $\Gamma_{\text {beam }}=I / 346$ (hour $\mu \mathrm{A}$ ), where $I$ is the average beam current. By varying the input parameters, an uncertainty of $40 \%$ was determined.

$\Gamma_{A F P}$ (AFP loss): During the production time, an AFP spin flip was performed every 20 min. The spin loss for each AFP was measured by performing many $(\sim 10)$ AFPs in a short time (several minutes). It was found that $\Gamma_{A F P} \approx$ $0.56 \% / 20$ minutes. 


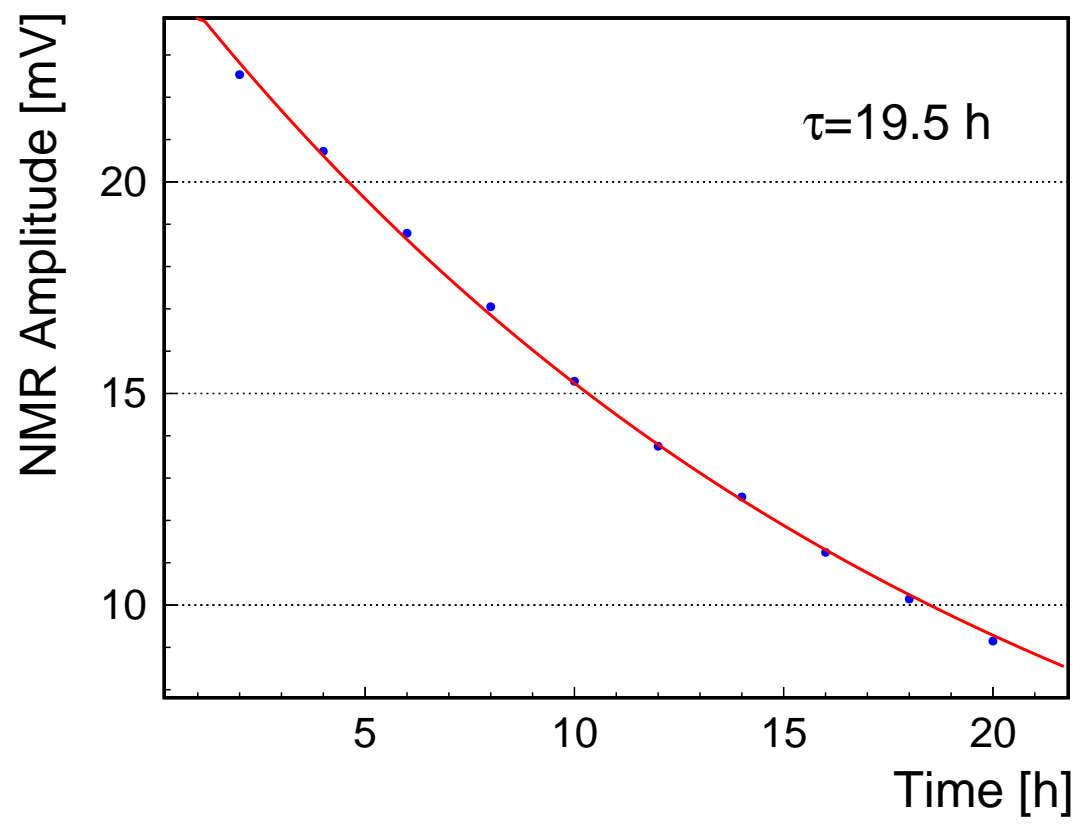

Figure 5.19: Target spin down curve at room temperature.

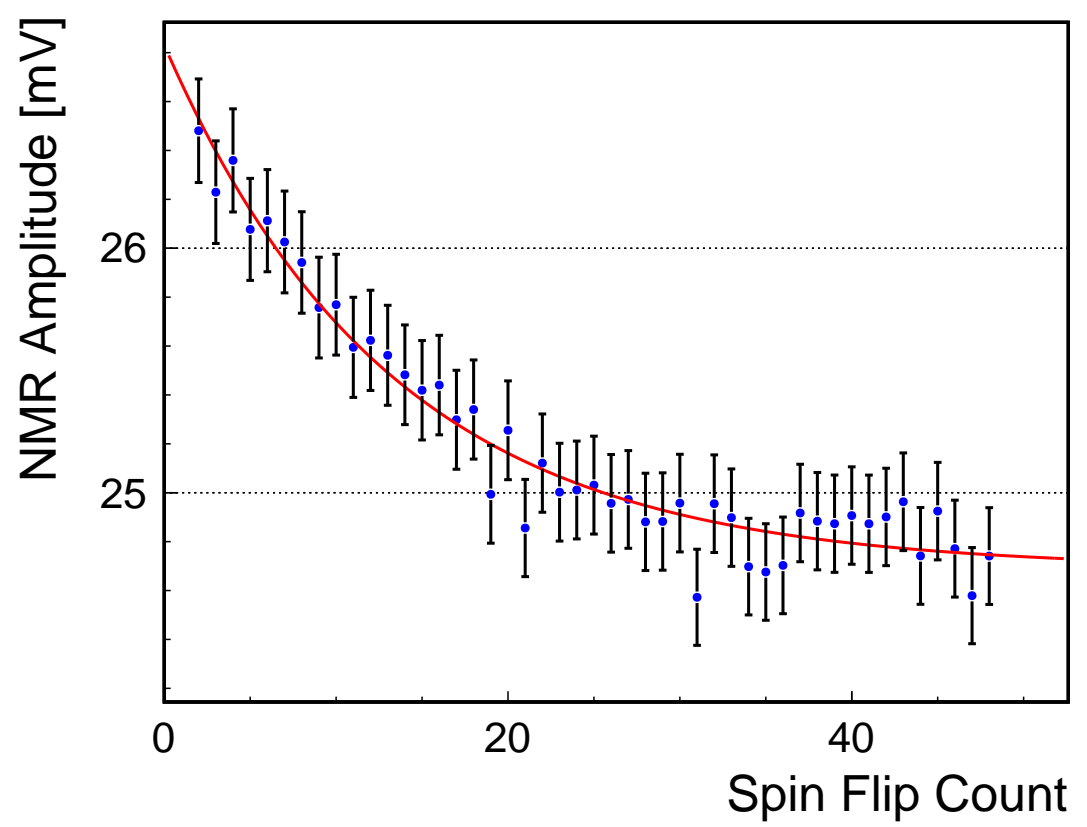

Figure 5.20: Beam depolarization effect. 


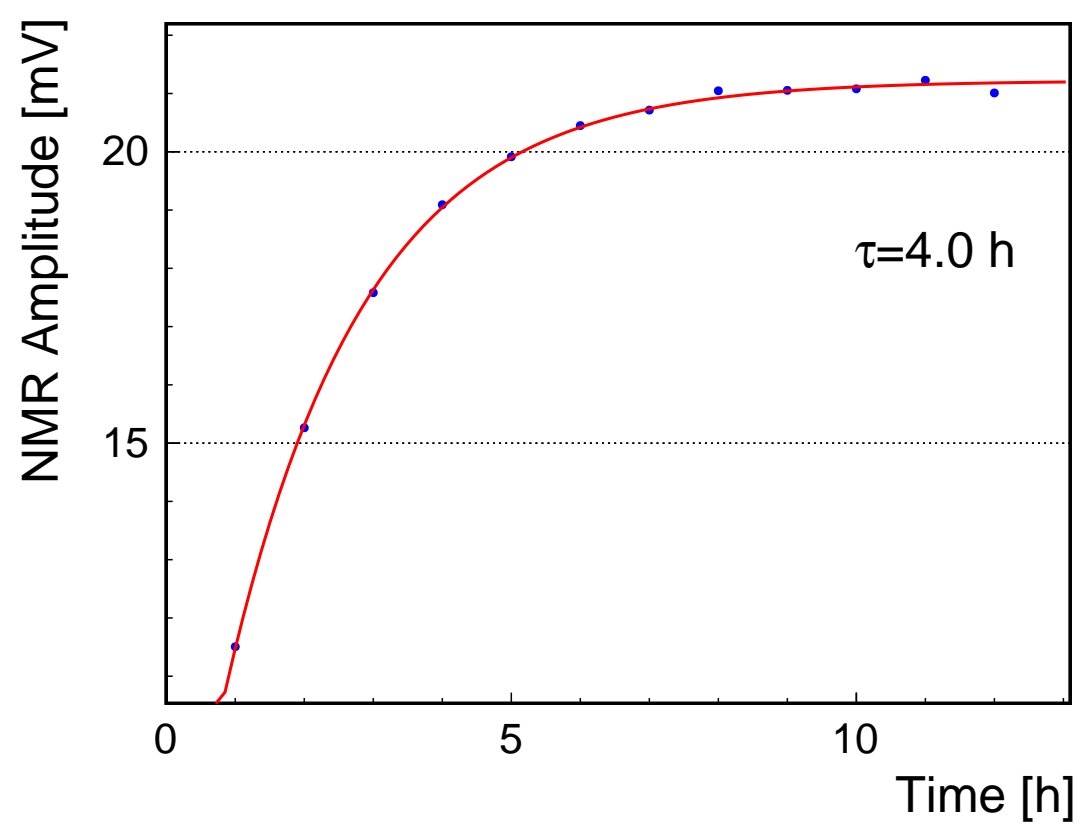

Figure 5.21: Spin up measurement for the cell Dominic.

$\Gamma_{\Delta B}$ (Magnetic field gradient): Relaxation due to the magnetic field gradient [74] 75 is given by

$$
\Gamma_{\Delta B}=D \frac{\left|\Delta B_{x}\right|^{2}+\left|\Delta B_{y}\right|^{2}}{B_{z}^{2}},
$$

where $\mathrm{D} \approx 0.2 \mathrm{~cm}^{2} / s$ is the ${ }^{3} \mathrm{He}$ self-diffusion coefficient. The value of $\Gamma_{\Delta B}$ $\approx\left(10^{3} \text { hour }\right)^{-1}$, which is very small and was neglected during our analysis.

\subsubsection{Polarization Gradient Result}

Based on Equation (5.37), the polarization gradient can be calculated with

$$
\begin{gathered}
\frac{P_{t c}^{\infty}}{P_{p c}^{\infty}}=\frac{1}{1+\frac{\Gamma_{t c}}{d_{t c}}} \\
\Gamma_{t c}=\Gamma_{\text {wall }}+\Gamma_{h e}^{t c}+\Gamma_{\text {beam }}+\Gamma_{A F P}+\Gamma_{\Delta B},
\end{gathered}
$$

The results in $P_{t c}^{\infty} / P_{p c}^{\infty}=0.845$, with an uncertainty of $4.3 \%$. 


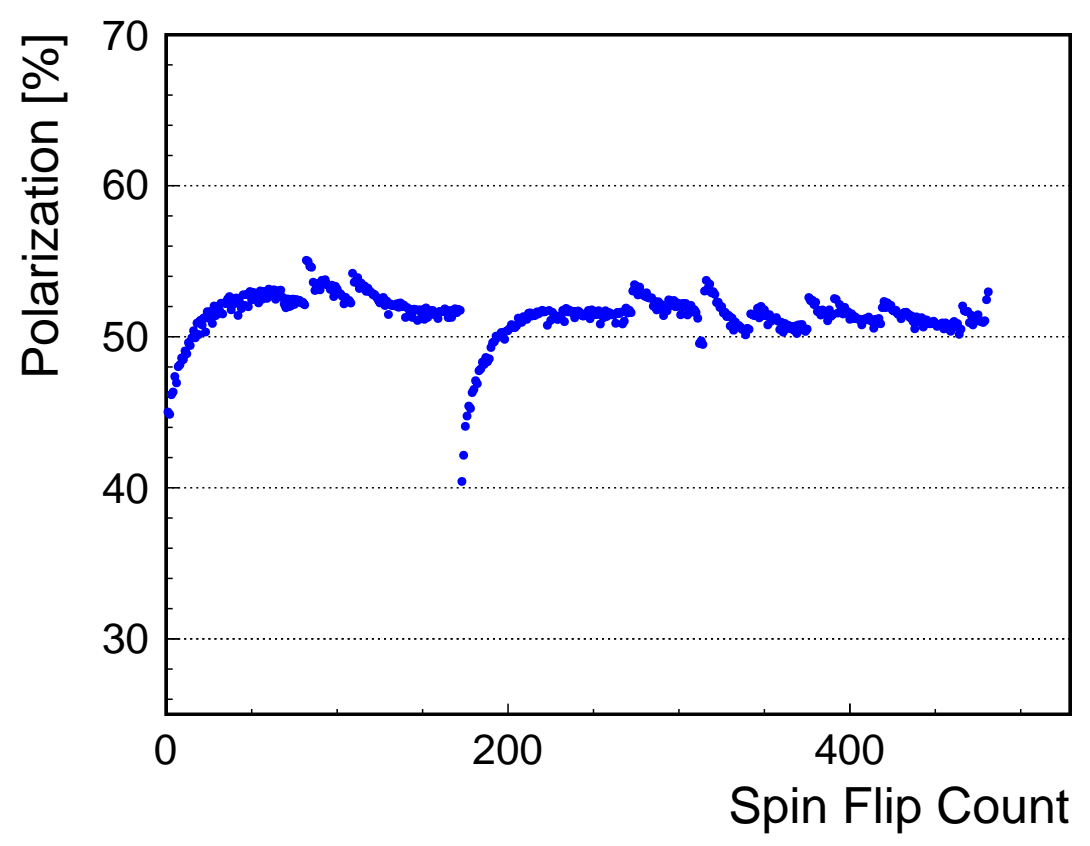

Figure 5.22: Target polarization versus spin flip.

\subsection{Target Performance}

The ${ }^{3} \mathrm{He}$ polarization in the target chamber is shown in Figure 5.22. The average

${ }^{3}$ He polarization in pumping chamber $\left(P_{p c}\right)$ and target chamber $\left(P_{t c}\right)$ are:

$$
\begin{aligned}
& <P_{\mathrm{pc}}>=60.6 \% \pm 0.5 \% \text { (average stat. per NMR) } \pm 2.2 \% \text { (sys.) } \\
& <P_{\mathrm{tc}}>=51.4 \% \pm 0.4 \% \text { (average stat. per NMR) } \pm 2.9 \% \text { (sys.). }
\end{aligned}
$$

The systematic uncertainties of the target polarization are summarized in Table 5.10 . 
Table 5.10: Systematic uncertainty budget for the target polarization.

\begin{tabular}{|c|c|c|}
\hline \multicolumn{4}{|c|}{ Items } & Rel.Pol.Error \\
\hline \multirow{3}{*}{ Pumping Chamber } & K- ${ }^{3}$ He EPR $\kappa_{0}$ & $2.8 \%$ \\
& Pumping chamber density & $2.1 \%$ \\
& EPR signal fit & $0.6 \%$ \\
& NMR signal fit & $0.8 \%$ \\
\hline \multirow{3}{*}{ Target Chamber } & Diffusion rate & $2.7 \%$ \\
& Target chamber intrinsic life time & $2.8 \%$ \\
& Beam depolarization & $1.7 \%$ \\
& Spin flip loss & $0.4 \%$ \\
\hline
\end{tabular}




\section{Chapter 6}

\section{Data Analysis}

The purpose of the data analysis is to extract the ${ }^{3} \mathrm{He}$ single-spin asymmetry, $A_{y}^{3} \mathrm{He}$, from ${ }^{3} \mathrm{He}^{\uparrow}\left(e, e^{\prime}\right)$ quasi-elastic scattering and to extract the corresponding neutron asymmetry $A_{y}^{\mathrm{n}}$ from $A_{y}^{3} \mathrm{He}$. A flow chart of the data analysis is shown in Figure 6.1. Using the standard Hall A analyzer package [76], developed by the Hall A staff and users, and the detector calibration database from the previous experiments, the raw data were processed and converted to ROOT files, which contain the reconstructed events from the detectors and scaler information. These primary ROOT files were used to check the scalers (Section 6.2) and calibrate the detectors (Section 6.3.3). Then, new ROOT files were created using updated calibration databases. By applying tracking and particle identification cuts to the raw data (Section 6.4), one can obtain the target-spin dependent electron counts: $N_{\uparrow}$ and $N_{\downarrow}$. These counts were then normalized to the accumulated charge $C$ and detector live-time $L$ gated by their respective target-spin states. These are referred to as the yields. The raw target single-spin asymmetry was formed from these two numbers (Equation (6.1)). The raw target single-spin asymmetry was further corrected for target polarization, nitrogen dilution (Section 6.7) and radiative corrections (Section 6.8). The result of the above analysis provides the physics asymmetry from polarized ${ }^{3} \mathrm{He}$ in the quasi-elastic region. The corresponding neutron single-spin asymmetry was extracted from the measured ${ }^{3} \mathrm{He}$ asymmetry using the effective polarization approximation (Section 6.9). 

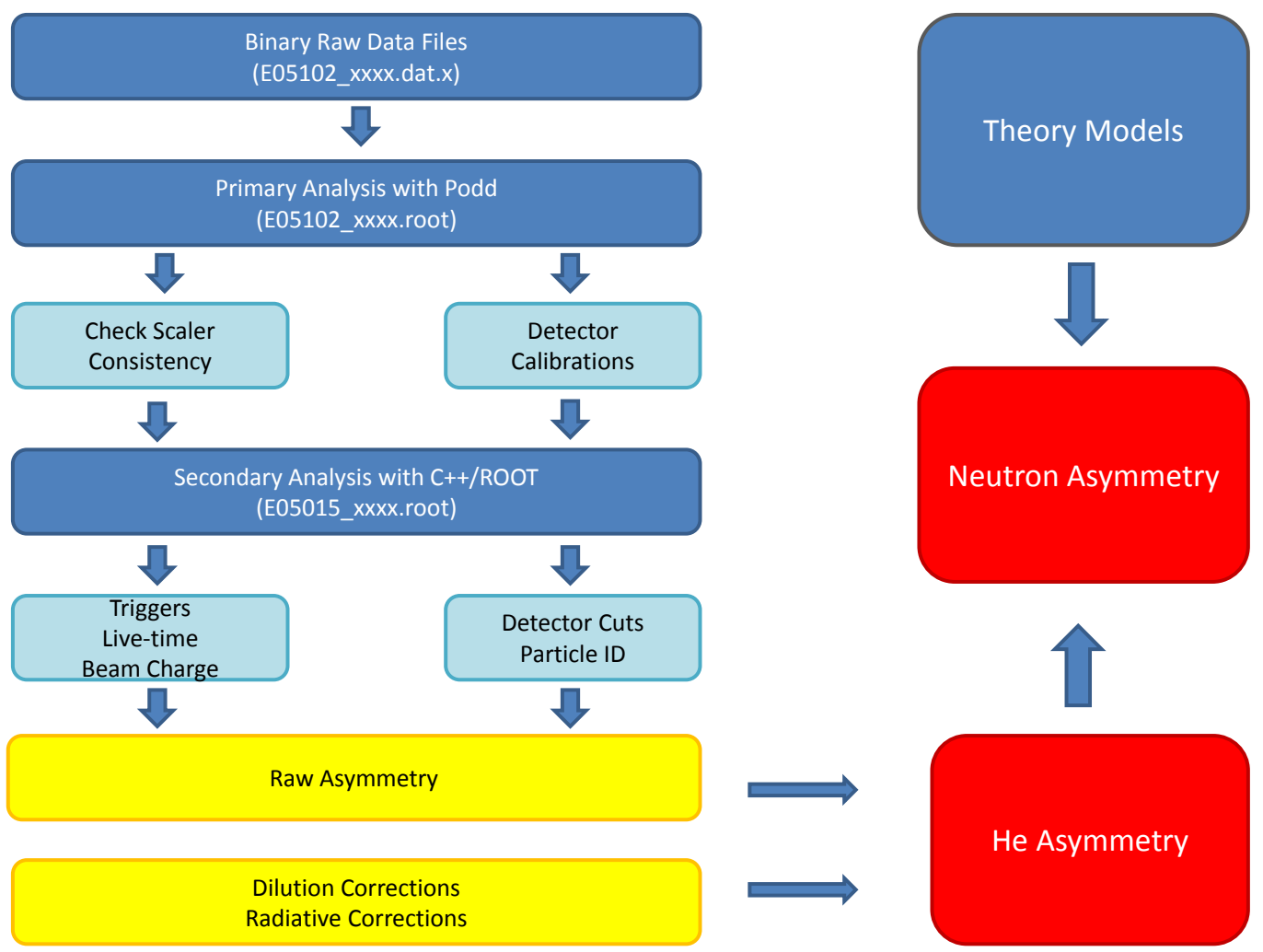

Dilution Corrections Radiative Corrections

Figure 6.1: The data analysis flow chart. 


\subsection{Asymmetry Extraction}

\subsubsection{Raw Asymmetry $A_{\text {raw }}$}

The raw target single spin asymmetry is defined as:

$$
A_{\text {raw }}=\frac{\sigma_{\uparrow}-\sigma_{\downarrow}}{\sigma_{\uparrow}+\sigma_{\downarrow}}=\frac{Y_{\uparrow}-Y_{\downarrow}}{Y_{\uparrow}+Y_{\downarrow}},
$$

where $\sigma_{\uparrow(\downarrow)}$ is the cross section of the target polarization vector in the vertically up (down) direction and $Y_{\uparrow(\downarrow)}$ is the yield of the corresponding target state. The yield is defined as

$$
Y=\frac{N}{C \cdot L}
$$

where $N$ is the number of events, $C$ is the charge and $L$ is the live-time. The livetime corrections include detector and electronics efficiencies as well as computer live time. Also implicit here is that other parameters are either independent of or uncorrelated to polarization direction so that differences average out. Examples of such parameters include spectrometer magnetic fields, target thickness, beam emittance, and beam position on target.

The uncertainty of the raw target single spin asymmetry is:

$$
\begin{aligned}
\delta A_{\text {raw }} & =\frac{2 a\left(N_{\downarrow} \delta N_{\uparrow}+N_{\uparrow} \delta N_{\downarrow}\right)}{\left(N_{\uparrow}+a N_{\downarrow}\right)^{2}} \\
& =\frac{2 a \sqrt{N_{\downarrow}^{2} N_{\uparrow}+N_{\uparrow}^{2} N_{\downarrow}}}{\left(N_{\uparrow}+a N_{\downarrow}\right)^{2}},
\end{aligned}
$$

where $a=\frac{C_{\uparrow} L_{\uparrow}}{C_{\downarrow} L_{\downarrow}}$.

\subsection{2 ${ }^{3}$ He Experimental Asymmetry $A_{e x p}^{3} \mathrm{He}$}

The yield, $Y$, is comprised of three main parts: ${ }^{3} \mathrm{He}$ yield $\left(Y^{3} \mathrm{He}\right), \mathrm{N}_{2}$ yield $\left(Y^{\mathrm{N}_{2}}\right)$ and empty target cell yield ( $\left.Y^{\text {empty }}\right)$. Equation (6.1) can therefore be rewritten 
as

$$
\begin{aligned}
& A_{\text {raw }}=\frac{Y_{\uparrow}^{3 \mathrm{He}}+Y^{\mathrm{N}_{2}}+Y^{\mathrm{empty}}-\left(Y_{\downarrow}^{3 \mathrm{He}}+Y^{\mathrm{N}_{2}}+Y^{\mathrm{empty}}\right)}{Y_{\uparrow}^{3} \mathrm{He}+Y^{\mathrm{N}_{2}}+Y^{\mathrm{empty}}+\left(Y_{\downarrow}^{3} \mathrm{He}+Y^{\mathrm{N}_{2}}+Y^{\mathrm{empty}}\right)}
\end{aligned}
$$

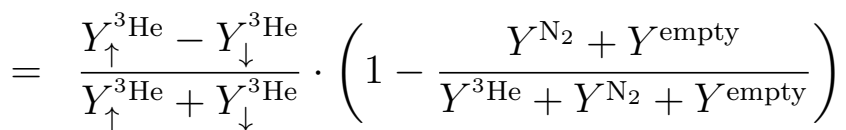

$$
\begin{aligned}
& =A_{\text {exp }}^{3} \mathrm{He} \cdot(1-f) \text {, }
\end{aligned}
$$

where $A_{\text {exp }}^{3} \mathrm{He}$ is the experimental asymmetry of ${ }^{3} \mathrm{He}$ and

$$
f=\frac{Y^{\mathrm{N}_{2}}+Y^{\mathrm{empty}}}{Y^{3} \mathrm{He}+Y^{\mathrm{N}_{2}}+Y^{\mathrm{empty}}}
$$

is the dilution factor from $\mathrm{N}_{2}$ and glass. Taking the ${ }^{3} \mathrm{He}$ target polarization $P$ into account, the expression of $A_{\text {exp }}^{3} \mathrm{He}$ in terms of the raw asymmetry, $A_{\text {raw }}$, is given by

$$
A_{\text {exp }}^{3} \mathrm{He}=\frac{A_{\text {raw }}}{(1-f) \cdot P} .
$$

\subsection{3 ${ }^{3} \mathrm{He}$ Experimental Asymmetry in the Quasi-Elastic Region $A_{e x p}^{q e}$}

In addition, the ${ }^{3} \mathrm{He}$ yield can also be divided into two separate parts: the elastic radiative tail $\left(Y^{e r t}\right)$ and quasi-elastic $\left(Y^{q e}\right)$ contributions:

$$
\begin{aligned}
A_{\text {exp }}^{3} \mathrm{He} & =\frac{Y_{\uparrow}^{3} \mathrm{He}-Y_{\downarrow}^{3} \mathrm{He}}{Y_{\uparrow}^{3} \mathrm{He}+Y_{\downarrow}^{3} \mathrm{He}} \\
& =\frac{Y_{\uparrow}^{\text {ert }}+Y_{\uparrow}^{q e}-\left(Y_{\downarrow}^{e r t}+Y_{\downarrow}^{q e}\right)}{Y_{\uparrow}^{\text {ert }}+Y_{\uparrow}^{q e}+Y_{\downarrow}^{\text {ert }}+Y_{\downarrow}^{q e}} \\
& =\left(1-R^{\text {ert }}\right) A_{\text {exp }}^{q e}+R^{\text {ert }} A_{\text {exp }}^{\text {ert }},
\end{aligned}
$$

where

$$
\begin{aligned}
& A_{\text {exp }}^{e r t}=\frac{Y_{\uparrow}^{e r t}-Y_{\downarrow}^{e r t}}{Y_{\uparrow}^{e r t}+Y_{\downarrow}^{e r t}} \\
& A_{\text {exp }}^{q e}=\frac{Y_{\uparrow}^{q e}-Y_{\downarrow}^{q e}}{Y_{\uparrow}^{q e}+Y_{\downarrow}^{q e}},
\end{aligned}
$$


and

$$
R^{e r t}=\frac{Y^{e r t}}{Y^{3 \mathrm{He}}}
$$

The expression of $A_{\text {exp }}^{q e}$ in terms of the ${ }^{3} \mathrm{He}$ experimental asymmetry, $A_{\text {exp }}^{3} \mathrm{He}$, is given by

$$
A_{e x p}^{q e}=\frac{A_{e x p}^{3} \mathrm{He}-R^{e r t} A_{e x p}^{e r t}}{1-R^{e r t}} .
$$

\subsubsection{Prescale Factor Correction}

For E05-015, the singles triggers were taken with prescale factors, $p$. For $p>$ 1 , this leads to a change in the statistical uncertainties of asymmetries. The derivation can be found in [77].

$$
S=\sqrt{1-L \cdot r\left(1-\frac{1}{p}\right)},
$$

where $L$ is the live-time, $r$ is the acceptance for useful events and $p$ is the prescale factor.

\subsection{Scaler Consistency Check}

During the experiment, scalers were used to record the accumulated charges and the raw number of trigger counts. The LHRS and RHRS were both used to collect the data, and all the important scaler signals were sent to both arms to be counted as a cross-check, which means there are two copies for the important scalers. The spectrometers were synchronized so that they would have the same run time for each production run pair. Then, ideally, the reading of a certain scaler from the left-arm should be the same as that from the right-arm. Since the signals were read out by different scaler modules. By comparing the scaler counts, we can easily identify problems. 
As we mentioned in Chapter 5, in each HRS, five scaler modules were installed to record five types of scaler readings. The "ungated" scaler represents the reading independent of the target spin and beam helicity states and the "gated" scalers represent four types of scaler readings $(++,+-,-+,-)$ from the possible combinations of target spin states $(+$ and -$)$ and beam helicity states $(+$ and -$)$. In this analysis, these important scalers were checked:

- main triggers: RHRS trigger t1 and LHRS trigger t3.

- charges: u1, u3, u10, d1, d3, d10 and Unser.

- clocks: $1 \mathrm{kHz}$ and $103.7 \mathrm{kHz}$.

The results of the scaler consistency check are summarized in Table6.1. It was found that the ungated scaler was the most reliable one. Therefore, the ungated scaler was used to determine the target spin dependent scalers according to the target spin state recorded for each event.

\subsection{Calibration}

The production running for the experiment was divided into three run periods based on different beam energies $(1.245,2.425$ and $3.605 \mathrm{GeV})$, corresponding to different $\mathrm{Q}^{2}$ points. All calibrations were done separately for these run periods except for the BCM calibration. In the following sections, only LHRS calibrations will be presented, since the procedures for the RHRS calibration were the same as those for LHRS.

\subsubsection{Beam Current Monitor Calibration}

The accumulated scaler counts of the BCM scalers corresponds to the collected charge, with the scaler rate corresponding to the electron beam current. The 
Table 6.1: Summary of the scaler consistency check results. " $\sqrt{ }$ " means consistent between LHRS and RHRS copies (scaler asymmetries $<2 \times 10^{-4}$ ), " $\times$ " means not consistent and it is not known which copy was bad, "Right" means the right HRS copy was bad.

\begin{tabular}{|c|ccccccccccc|}
\multicolumn{110}{c|}{ E $=1.245 \mathrm{GeV}$} \\
\hline (Target,Beam) & $\mathrm{t} 1$ & $\mathrm{t} 3$ & $\mathrm{u} 1$ & $\mathrm{u} 3$ & $\mathrm{u} 10$ & $\mathrm{~d} 1$ & $\mathrm{~d} 3$ & $\mathrm{~d} 10$ & Unser & clk & fclk \\
\hline Ungated & $\sqrt{ }$ & $\sqrt{ }$ & $\sqrt{ }$ & $\sqrt{ }$ & $\sqrt{ }$ & $\times$ & $\times$ & $\sqrt{ }$ & $\sqrt{ }$ & $\sqrt{ }$ & $\sqrt{ }$ \\
++ & $\times$ & $\times$ & $\times$ & $\times$ & $\times$ & $\times$ & $\times$ & $\times$ & $\times$ & $\times$ & $\times$ \\
+- & $\times$ & $\sqrt{ }$ & $\sqrt{ }$ & $\sqrt{ }$ & $\sqrt{ }$ & $\times$ & $\times$ & $\sqrt{ }$ & $\sqrt{ }$ & $\sqrt{ }$ & $\sqrt{ }$ \\
-+ & $\sqrt{ }$ & $\sqrt{ }$ & $\sqrt{ }$ & $\sqrt{ }$ & $\sqrt{ }$ & $\times$ & $\times$ & $\sqrt{ }$ & $\sqrt{ }$ & $\times$ & $\sqrt{ }$ \\
-- & $\sqrt{ }$ & $\sqrt{ }$ & $\sqrt{ }$ & $\sqrt{ }$ & $\sqrt{ }$ & $\times$ & $\times$ & $\sqrt{ }$ & $\sqrt{ }$ & $\times$ & $\sqrt{ }$ \\
\hline
\end{tabular}

\begin{tabular}{|c|cccccccccccc|}
\multicolumn{110}{|c|}{ (Target,Beam) } & $\mathrm{t} 1$ & $\mathrm{t} 3$ & $\mathrm{u} 1$ & $\mathrm{u} 3$ & $\mathrm{u} 10$ & $\mathrm{~d} 1$ & $\mathrm{~d} 3$ & $\mathrm{~d} 10$ & Unser & clk & fclk \\
\hline Ungated & $\sqrt{ }$ & Right & $\sqrt{ }$ & $\sqrt{ }$ & $\sqrt{ }$ & $\times$ & $\sqrt{ }$ & $\sqrt{ }$ & $\sqrt{ }$ & $\sqrt{ }$ & $\sqrt{ }$ \\
++ & $\times$ & $\times$ & $\times$ & $\times$ & $\times$ & $\times$ & $\times$ & $\times$ & $\times$ & $\times$ & $\times$ \\
+- & $\sqrt{ }$ & $\sqrt{ }$ & $\sqrt{ }$ & $\sqrt{ }$ & $\sqrt{ }$ & $\times$ & $\sqrt{ }$ & $\sqrt{ }$ & $\sqrt{ }$ & $\sqrt{ }$ & $\sqrt{ }$ \\
-+ & $\sqrt{ }$ & $\sqrt{ }$ & $\sqrt{ }$ & $\sqrt{ }$ & $\sqrt{ }$ & $\times$ & $\sqrt{ }$ & $\sqrt{ }$ & $\sqrt{ }$ & $\sqrt{ }$ & $\sqrt{ }$ \\
-- & $\sqrt{ }$ & $\sqrt{ }$ & $\sqrt{ }$ & $\sqrt{ }$ & $\sqrt{ }$ & $\times$ & $\times$ & $\sqrt{ }$ & $\sqrt{ }$ & $\times$ & $\sqrt{ }$ \\
\hline
\end{tabular}

$$
\mathrm{E}=3.605 \mathrm{GeV}
$$

\begin{tabular}{|c|ccccccccccc|}
\hline (Target,Beam) & $\mathrm{t} 1$ & $\mathrm{t} 3$ & $\mathrm{u} 1$ & $\mathrm{u} 3$ & $\mathrm{u} 10$ & $\mathrm{~d} 1$ & $\mathrm{~d} 3$ & $\mathrm{~d} 10$ & Unser & clk & fclk \\
\hline Ungated & $\sqrt{ }$ & Right & $\sqrt{ }$ & $\sqrt{ }$ & $\sqrt{ }$ & $\times$ & $\sqrt{ }$ & $\sqrt{ }$ & $\sqrt{ }$ & $\sqrt{ }$ & $\sqrt{ }$ \\
++ & $\times$ & $\sqrt{ }$ & $\sqrt{ }$ & $\sqrt{ }$ & $\sqrt{ }$ & $\times$ & $\sqrt{ }$ & $\sqrt{ }$ & $\sqrt{ }$ & $\times$ & $\sqrt{ }$ \\
+- & $\times$ & $\sqrt{ }$ & $\sqrt{ }$ & $\sqrt{ }$ & $\sqrt{ }$ & $\times$ & $\sqrt{ }$ & $\sqrt{ }$ & $\sqrt{ }$ & $\sqrt{ }$ & $\sqrt{ }$ \\
-+ & $\sqrt{ }$ & $\sqrt{ }$ & $\sqrt{ }$ & $\sqrt{ }$ & $\sqrt{ }$ & $\times$ & $\sqrt{ }$ & $\sqrt{ }$ & $\sqrt{ }$ & $\times$ & $\sqrt{ }$ \\
-- & $\sqrt{ }$ & $\sqrt{ }$ & $\sqrt{ }$ & $\sqrt{ }$ & $\sqrt{ }$ & $\times$ & $\times$ & $\sqrt{ }$ & $\sqrt{ }$ & $\times$ & $\sqrt{ }$ \\
\hline
\end{tabular}


Table 6.2: Summary of calibration constants $k_{i}$ and offsets $b_{i}$ for all six BCMs.

\begin{tabular}{|c|cc|}
\hline $\begin{array}{c}\text { BCM Signal } \\
i\end{array}$ & $\begin{array}{c}\text { Calibration Constant } \\
\mathrm{k}_{i}\end{array}$ & $\begin{array}{c}\text { Offset } \\
\mathrm{b}_{i}\end{array}$ \\
\hline $\mathrm{u} 1$ & 2067.7 & 396.1 \\
$\mathrm{u} 3$ & 6370.9 & 490.0 \\
$\mathrm{u} 10$ & 19394.0 & 930.1 \\
\hline $\mathrm{d} 1$ & 2172.3 & 170.2 \\
$\mathrm{~d} 3$ & 6712.8 & 212.0 \\
$\mathrm{~d} 10$ & 21036.1 & 548.6 \\
\hline
\end{tabular}

beam current and charge can be obtained from the BCM scaler counts by:

$$
\begin{gathered}
I_{i}=\frac{d C_{i}}{d t}=\frac{\frac{N_{i}}{t}-b_{i}}{k_{i}} \\
C_{i}=I_{i} t=\frac{N_{i}-b_{i} t}{k_{i}},
\end{gathered}
$$

where $i=1,3,10$ is the gain factor (Section 4.4.2), $C$ is the charge, $t$ is the time period for each run and $N_{i}$ is the BCM scaler counts for each gain factor. The calibration constants $k_{i}$ and BCM offsets $b_{i}$ were determined as described below.

The electron beam current from the injector is precisely measured. The value was written into the Hall A data-stream as an EPICS variable (IBC0L02). During the beam current calibrations, the electron beam was only delivered to Hall A, and the beam current is adjusted in a step-function pattern between zero and various values. During this time, the beam current value from the injector point and the corresponding scaler counts for every BCM signal were recorded in the data-stream. Using Equation (6.18) with all the information above, the calibration constants $k_{i}$ and offsets $b_{i}$ were determined. Figure 6.2 shows the off-line calibration of the u3 BCM with the signal from "IBC0L02". The same procedure was used in calibrating all 6 BCMs (u1, u3, u10, d1, d3 and d10) and the results are shown in Table 6.2. 


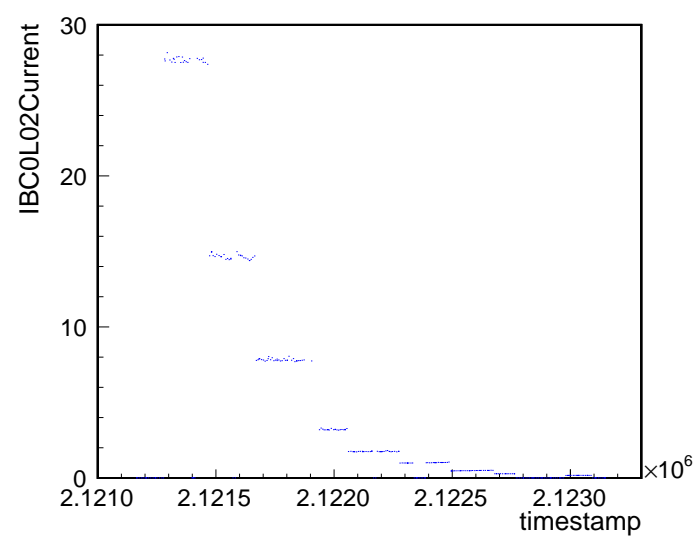

(a)

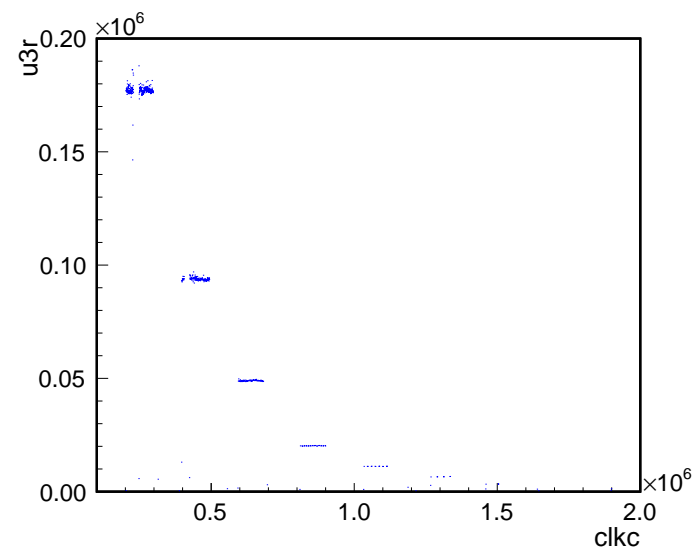

(b)

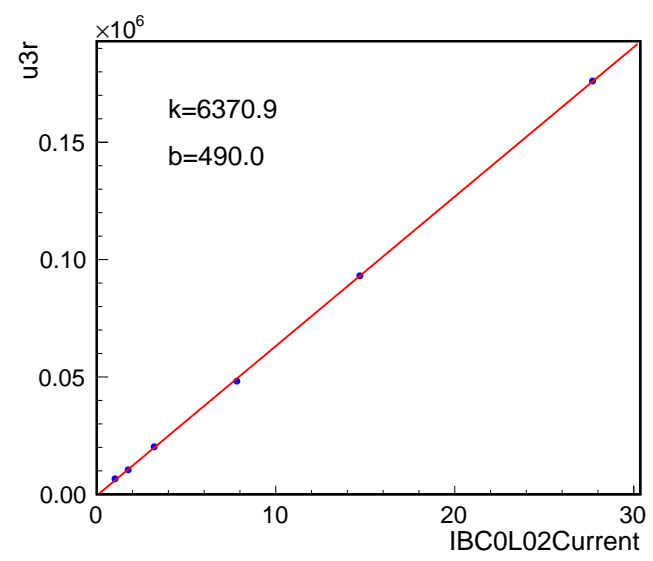

(c)

Figure 6.2: The calibration of the $\mathrm{u} 3 \mathrm{BCM}$ with the beam current value from the injector (IBC0L02). (a) The "IBC0L02" reading vs. the clock. (b) The $u 3 r$, rates of $u 3$, vs. the clock from the scalers. Constant fits were used to determine the reading of "IBC0L02" and $u 3 r$ at fixed beam currents. (c) The results from the previous two fits were fit with Equation (6.18). 


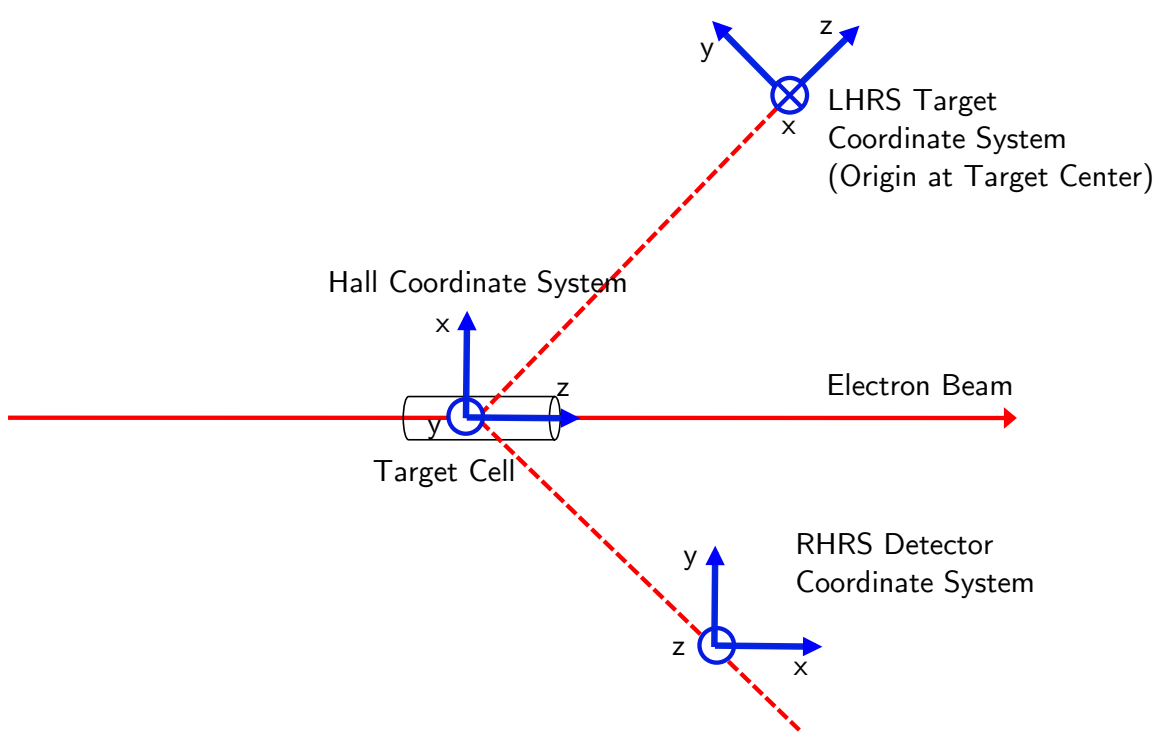

Figure 6.3: A schematic of three coordinate systems in common use in Hall A (top view). Electron beam is from left to right side in the figure.

\subsubsection{Optics Reconstruction}

The HRS optics calibration for experiment E05-015 was carried out by G. Jin [78] from University of Virginia. The goal was to reconstruct the target coordinate variables from the focal-plane variables.

\section{Coordinate Systems}

The trajectory of a particle can be described in different coordinate systems described in the following:

- Hall Coordinate System (HCS): The origin is defined as the center of the Hall. $\hat{z}$ points downstream along the beam, $\hat{x}$ is to the left side of the beam and $\hat{y}$ is vertically up. Note that the center of the Hall is not necessarily the center of the target.

- Target Coordinate System (TCS): The origin is defined as the center of the target. $\hat{z}$ is parallel to the spectrometer central ray, $\hat{y}$ is horizontal and points to the left (large angle) side of the spectrometer, and $\hat{x}$ is vertically 
down. Ideally, the central ray of the spectrometer should cross the Hall center, but in reality it does not. The deviation is surveyed to determine the pointing offset of the spectrometer.

- Detector Coordinate System (DCS): The origin is at the u1 plane of the $1^{\text {st }}$ chamber, at intersection of wires u1-184 and v1-184. It defines the position and direction of the trajectory of a particle at the VDC planes by the detector coordinate variables $x_{d e t}, y_{d e t}, \theta_{d e t}$ and $\phi_{d e t}$.

- Focal Plane Coordinate System (FCS): It is obtained by rotating the DCS around its $\hat{y}$ axis by an angle $\delta$, which is the angle between local central ray and the $\hat{z}$ axis of the DCS.

\section{Calibrations}

The details of the calibration procedure can be found in [78]. A brief summary of the process is presented as below:

The multi-foil carbon target is used to generate scattered particles at several distinct $z$ positions in the hall coordinate system. The target consists of seven $\mathrm{C}$ foils, which are spaced evenly along the $40 \mathrm{~cm}$ long target range. This is necessary because, as we mentioned in chapter 5 , the ${ }^{3} \mathrm{He}$ target we used was a $40-\mathrm{cm}$ long target instead of a point target. The reaction vertex $z_{\text {react }}$ was calibrated with reference to the carbon foil positions from the survey report. As shown in Figure 6.4, it provides a clear reaction point position along the $\hat{z}$ direction in the HCS.

The sieve slit plate $(0.5 \mathrm{~cm}$ thick tungsten $)$ was used to calibrate the direction of the trajectory of a particle, i.e., out-of-plane angle $\theta_{t g}$ and in-plane angle $\phi_{t g}$. In Hall A, each HRS is equipped with a set of sieve collimators placed $\sim 1.1 \mathrm{~m}$ away from the target center along the spectrometer central ray. Each sieve collimator has a pattern of 49 holes $(7 \times 7)$ that have a radius of $1 \mathrm{~mm}$ and are spaced 25 


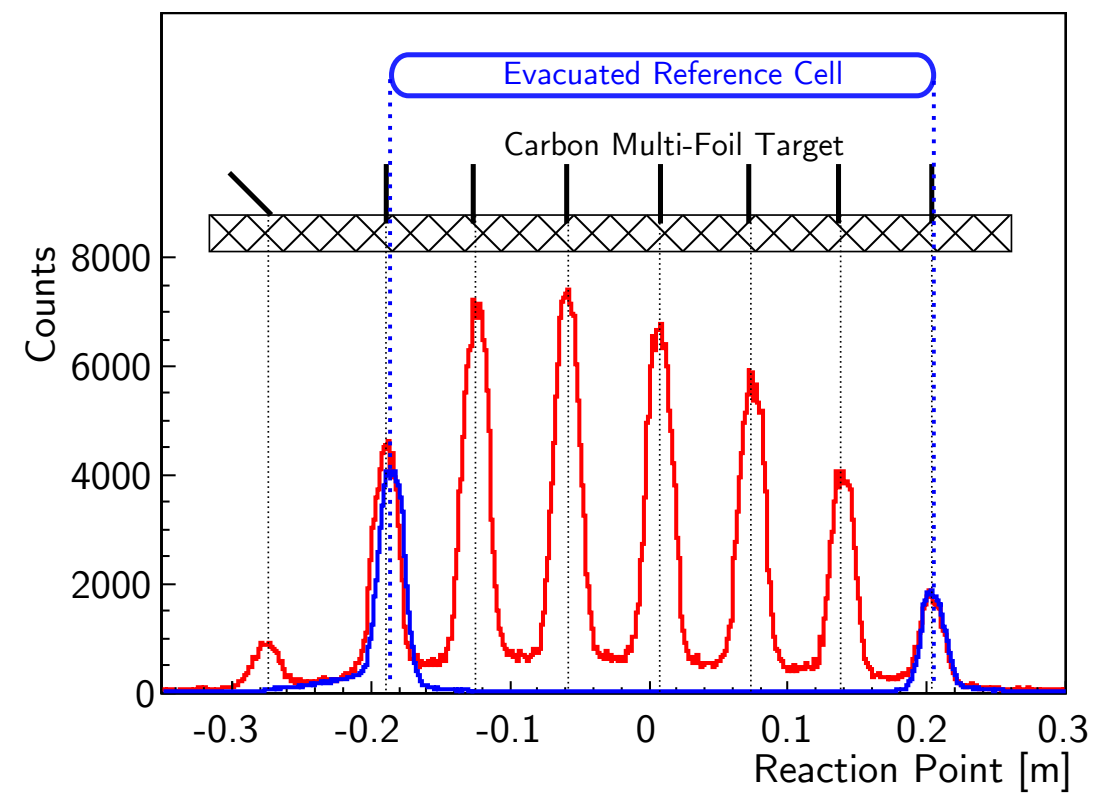

Figure 6.4: LHRS reconstructed vertex position. The reconstructed multi-foil carbon positions are shown in red. The dotted black lines show the actual position of the carbon foils. The blue line shows the evacuated reference cell data for comparison. At the left side of the plot, a slanted BeO foil is also shown. 

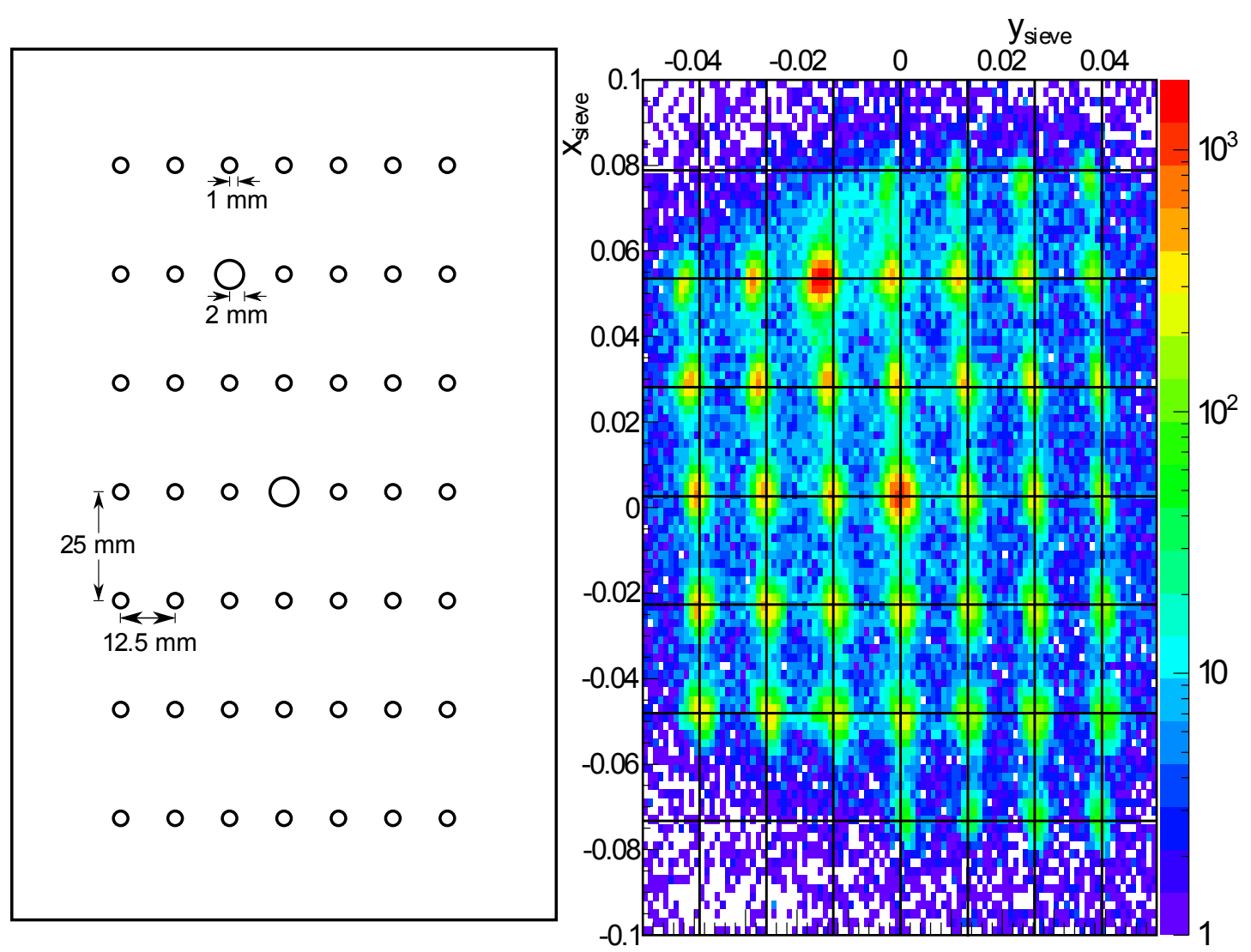

Figure 6.5: The schematic on the left shows the orientation of the sieve pattern used to calibrate the HRS. The plot on the right shows data with the sieve plate in after the calibration was completed.

mm apart vertically and $12.5 \mathrm{~mm}$ apart horizontally. Two of the holes have a radius of $2 \mathrm{~mm}$ and are placed in an asymmetric pattern such that the direction of the sieve can be easily determined at the focal plane. A sieve pattern diagram and the corresponding calibration result diagram are shown in Figure 6.5.

\subsubsection{Detector Calibration}

Gas Cherenkov Detector: As mentioned in Section 4.5.4, there are 10 PMTs in each Cherenkov detector. The calibration aligns the single photonelectron peak of each PMT to a certain ADC channel (200 in this case). The purpose of this alignment was to make sure that all the events that 
were not electrons, and thus were assumed to be mostly pions, were located at one specific ADC channel. The electrons, which were usually at high ADC channels, can then be cleanly separated from the non-electrons. Figure 6.6 shows the spectrum of the sum of all ten PMTs of the LHRS Cherenkov detector after calibration for the different run periods. For different run periods, a few coefficients were slightly adjusted to fit the detector performance best in each run period, although most coefficients were stable in the entire production data set.

Lead-Glass Calorimeter: During the calibration, the pion peaks in the ADCs for pion rejecter 1 were aligned to channel 100, and for pion rejecter 2 were aligned to channel 200. Figure 6.7 shows the energy deposited in the pion rejecter layer 1 vs. the energy deposited in the pion rejecter layer 2 after calibration. A clear separation of pions and electrons was achieved by using a $2-\mathrm{D}$ cut as shown in Figure 6.7

\subsection{Event Selection}

The events used in the asymmetry extraction were filtered through a series of cuts. The purpose of this was to select good electron events from all of the recorded events.

Beam Trip Cut: During the data collection, sometimes the beam current suddenly dropped to zero due to transient difficulties in the accelerator referred to as beam trips. Afterward the beam current is slowly ramped back to the set value. These beam trip periods were simply cut out and discarded in the analysis. An example of a beam trip cut is shown in Figure 6.8.

Trigger Type Cut: For each detected event, the trigger-type variable reports which triggers were present. For the left HRS, a cut on the main left HRS 


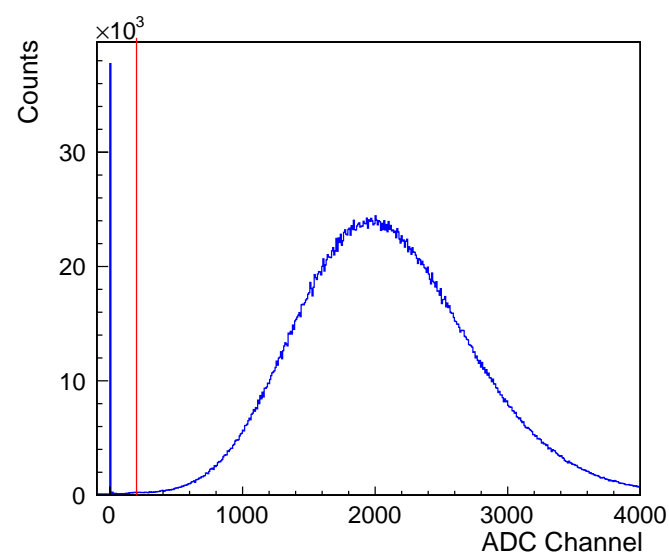

(a)

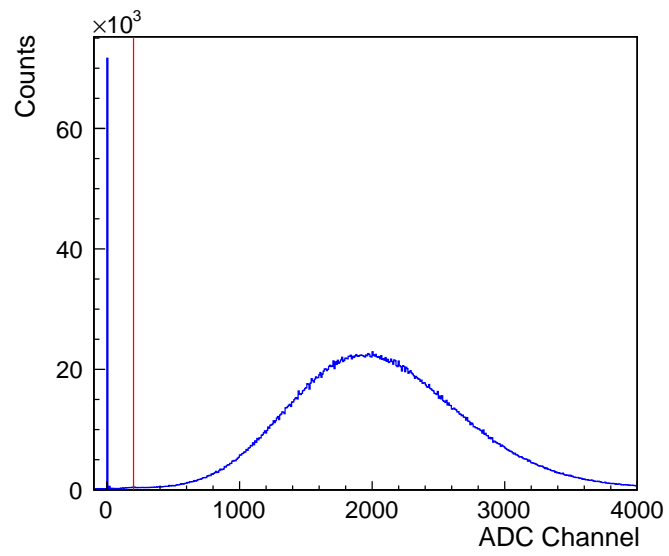

(b)

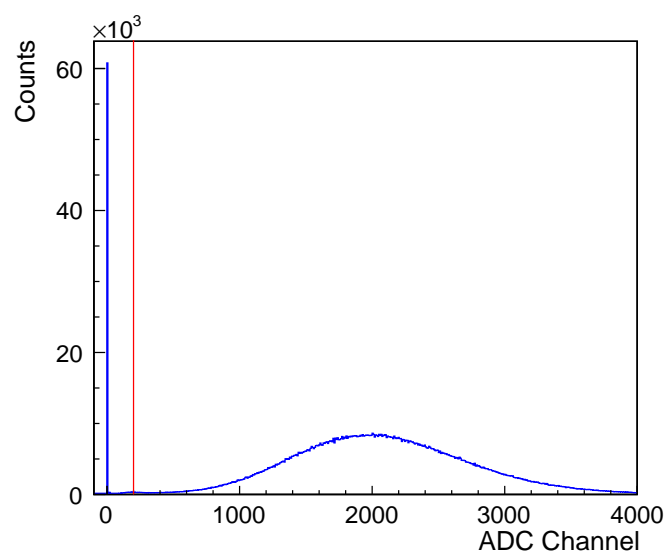

(c)

Figure 6.6: Spectra for the LHRS Gas Cherenkov detector after calibration. (a) $\mathrm{E}=1.245 \mathrm{GeV}$ (b) $\mathrm{E}=2.425 \mathrm{GeV}$ (c) $\mathrm{E}=3.605 \mathrm{GeV}$. The 10 PMT spectra are summed together. The single photon-electron peak is marked with a red line. 


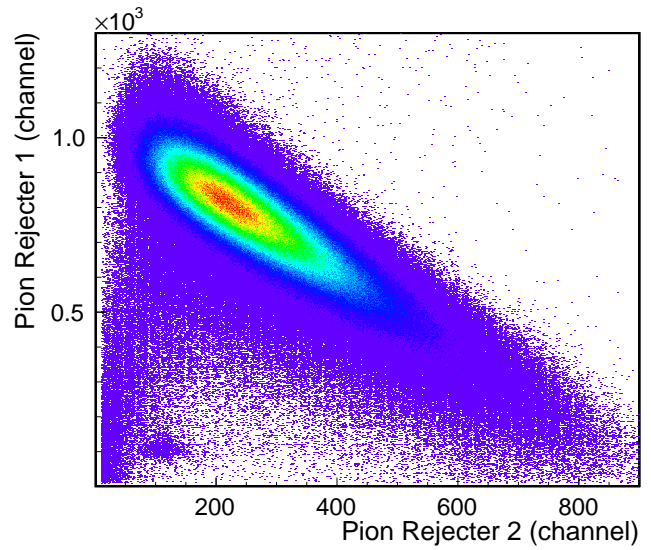

(a)

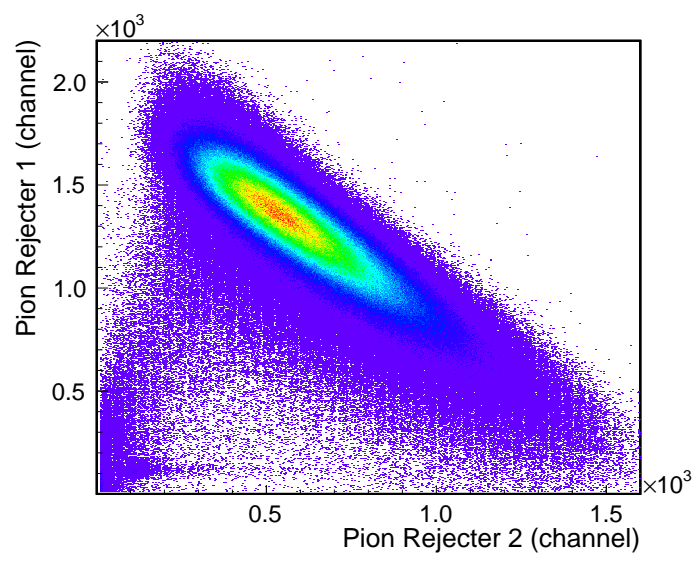

(b)

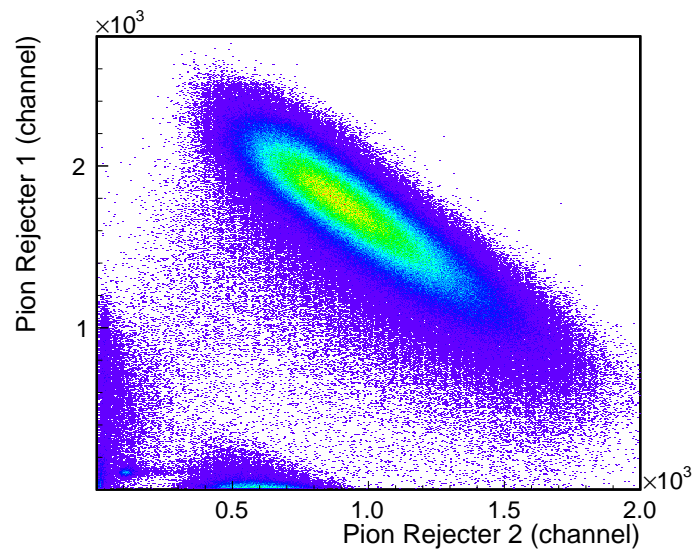

(c)

Figure 6.7: Energy deposited in the pion rejecters after calibration. (a) $E=1.245$ $\mathrm{GeV}$ (b) $\mathrm{E}=2.425 \mathrm{GeV}$ (c) $\mathrm{E}=3.605 \mathrm{GeV}$. 


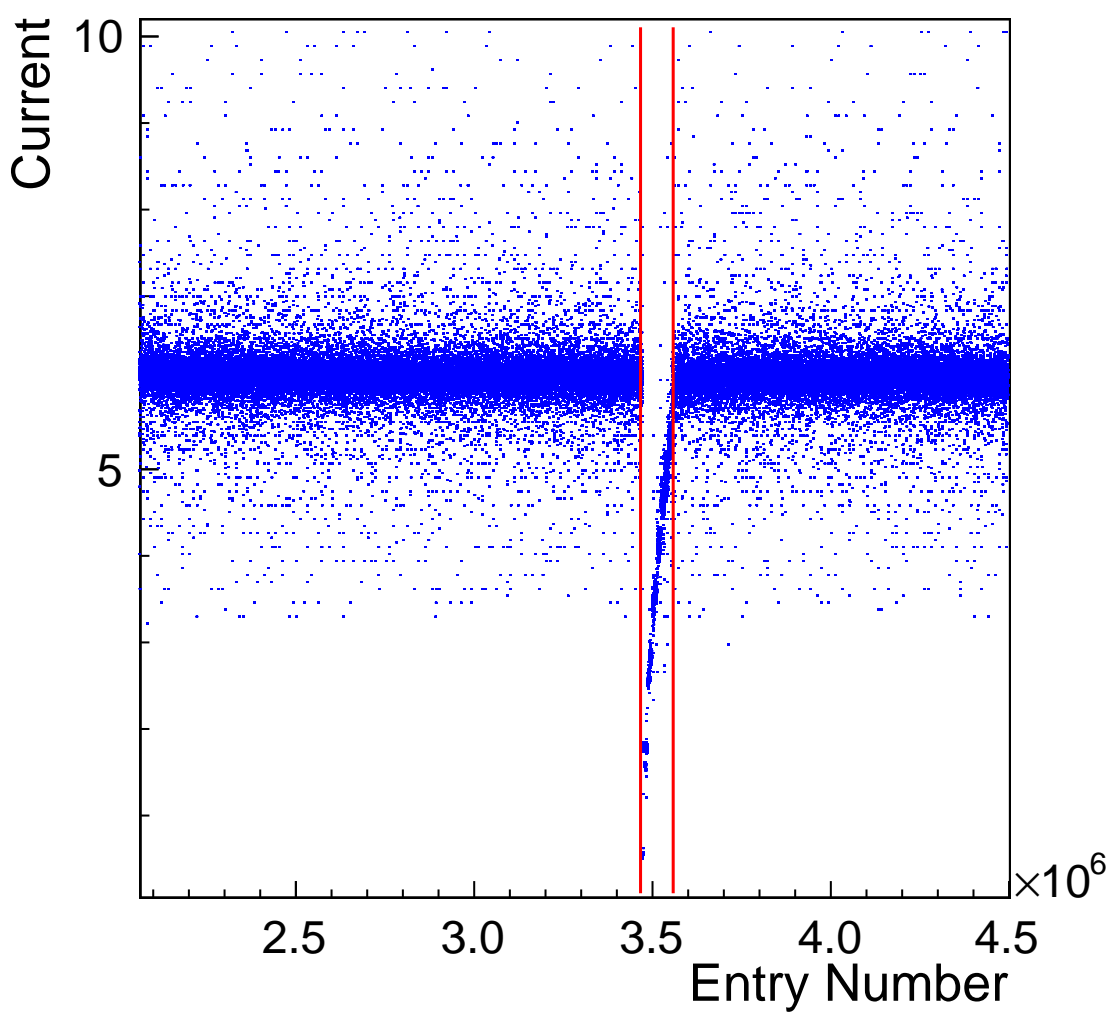

Figure 6.8: An example of beam trip cut. The events between the red lines were cut out and discarded during the analysis. 
trigger t3 was placed, and for the right HRS, a cut on the main right HRS trigger $\mathrm{t} 1$ was made. The programming code used was of the following form:

LHRS: DL.evtypebits $\&(1<<3)==(1<<3)$

RHRS: D.evtypebits \& $(1<<1)==(1<<1)$.

Spectrometer Acceptance Cut: Simplified cuts were used to remove events far away from the central acceptance, since in these events there can be re-scattering at the edges of the acceptance. The cuts for the left HRS at $E=1.245 \mathrm{GeV}$, shown in Figure 6.9, were placed on six 2-D projections of the 4-D acceptance space.

VDC Single Track Cut: One and only one track should exist as shown in Figure 6.10 for the HRS spectrometers, thus $N_{\text {track }}=1$. The programming code used was of the following form:

LHRS: L.tr.n==1

RHRS: R.tr.n==1.

Vertex z Cut: The vertex cut was applied to exclude events from the glass target windows at $\pm 20 \mathrm{~cm}$. The position of the reaction vertex along the beam direction is given by the variable $z_{\text {react }}$, which was calibrated individually for each spectrometers. To determine the best possible position of the cuts, the ${ }^{3} \mathrm{He}$ target cell data was compared to the empty target cell data. The comparison is presented in Figure 6.12 (a). We first found the position of the two glass windows and their width $(\sigma)$, then reduced the cut one $\sigma$ at a time to compare the glass wall contamination to the ${ }^{3} \mathrm{He}$ events. The results are shown in Figure 6.12 (b). According to this analysis, the optimal cut was $\approx 3 \sigma$ away from the cell walls as shown in Figure 6.11.

LHRS: $\mathrm{z}_{\text {up }}+3 \sigma_{\text {up }} \leq$ ReactPt_L.z $\leq \mathrm{z}_{\text {down }}-3 \sigma_{\text {down }}$

RHRS: $\mathrm{z}_{\text {up }}+3 \sigma_{\text {up }} \leq$ ReactPt_R.z $\leq \mathrm{z}_{\text {down }}-3 \sigma_{\text {down }}$, 


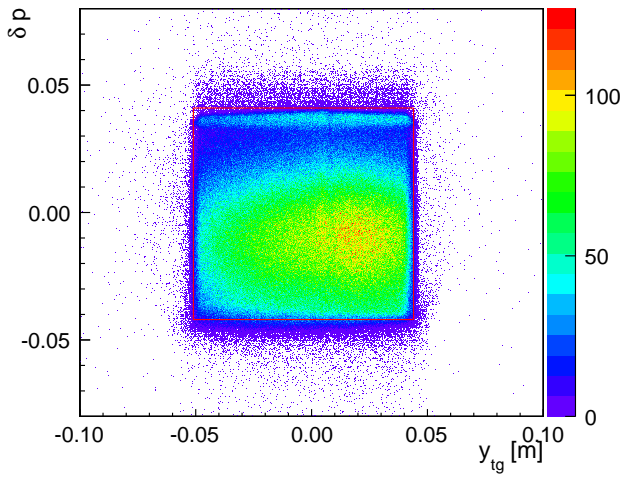

(a)

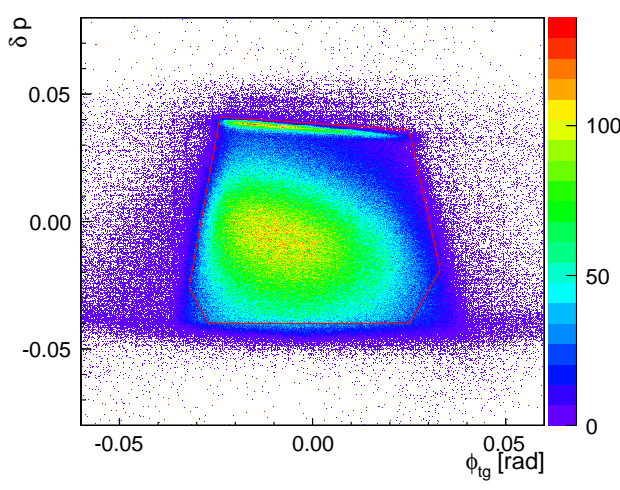

(c)

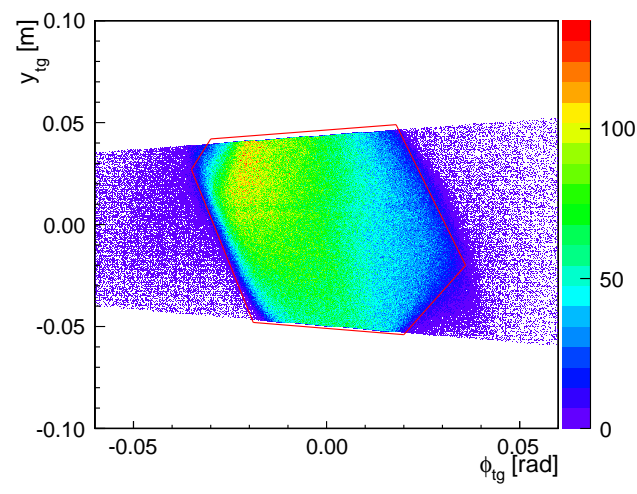

(e)

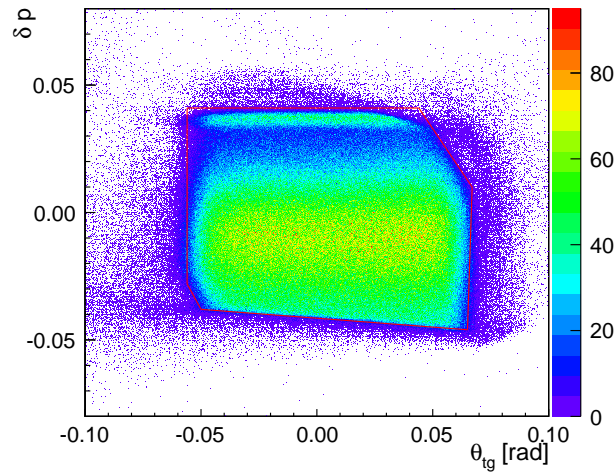

(b)

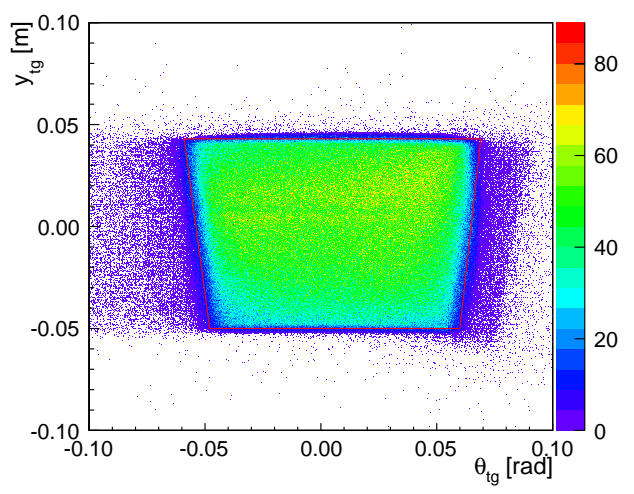

(d)

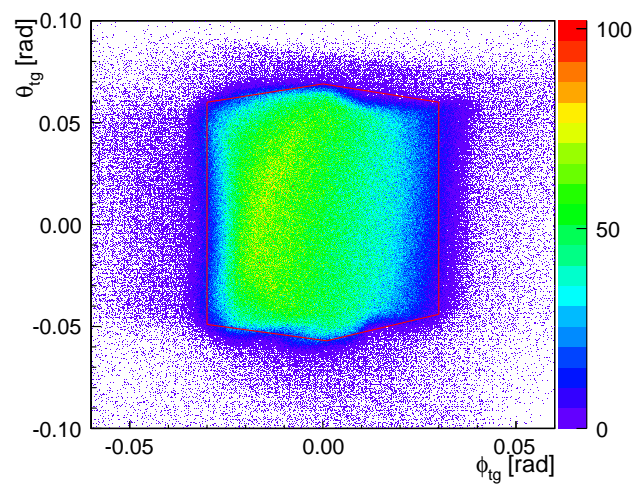

(f)

Figure 6.9: Left HRS geometry cuts (red lines) at $\mathrm{E}=1.245 \mathrm{GeV}$. 


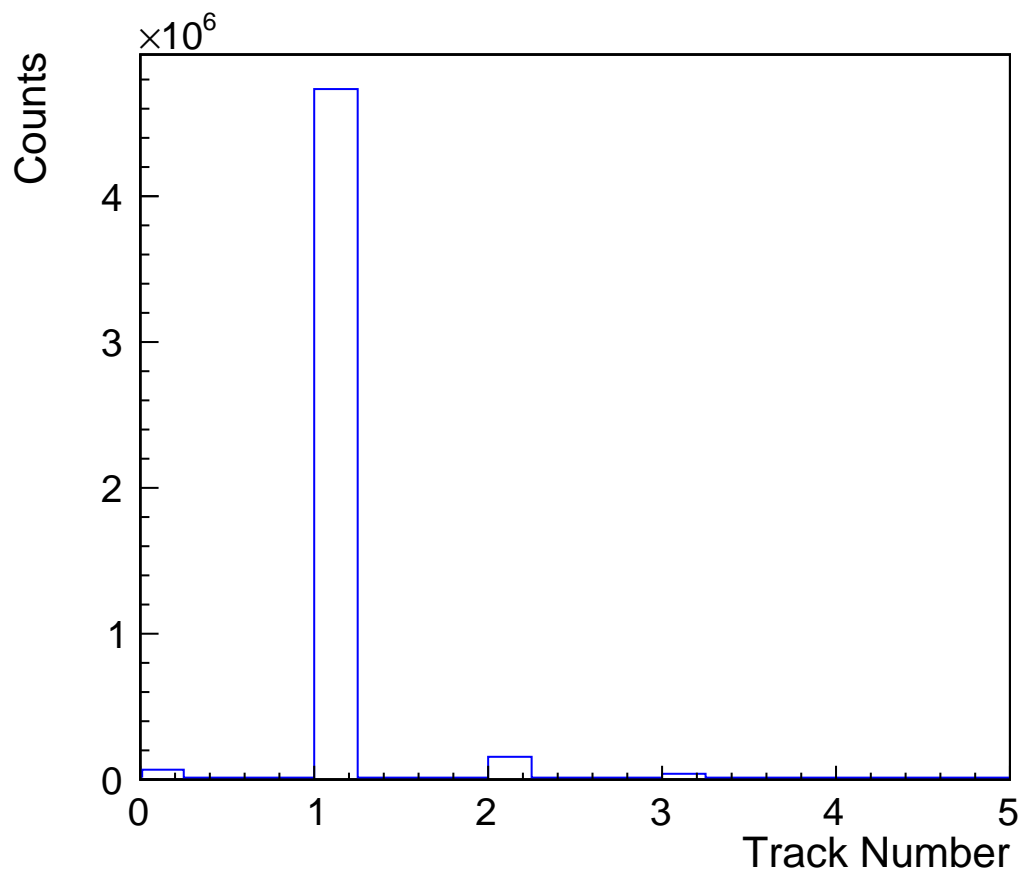

Figure 6.10: An example of track number.

Table 6.3: Summary of parameters used for vertex cuts. All quantities in units of $\mathrm{m}$.

\begin{tabular}{|c|cccc|}
\hline Spectrometer & $\mathrm{z}_{\text {up }}$ & $\sigma_{\text {up }}$ & $\mathrm{Z}_{\text {down }}$ & $\sigma_{\text {down }}$ \\
\hline LHRS & -0.186 & 0.010 & 0.205 & 0.010 \\
RHRS & -0.195 & 0.011 & 0.196 & 0.010 \\
\hline
\end{tabular}

where $z_{\mathrm{up}}$ and $z_{\text {down }}$ represent the position of the upstream and downstream cell windows, while $\sigma_{\text {up }}$ and $\sigma_{\text {down }}$ are their reconstructed root-mean-square widths. A summary of the parameters used for the vertex cuts is shown in Table 6.3.

Gas Cherenkov Detector Cut: Electrons generate Cerenkov light leading to signals in the Cherenkov detector, whereas hadrons do not generate real signals, though low-level noise can be present. Thus cuts on the left Cherenkov $\mathrm{ADC}>400$ and the right Cherenkov ADC $>350$ were placed.

LHRS: L.cer.asum_c $>400$ 


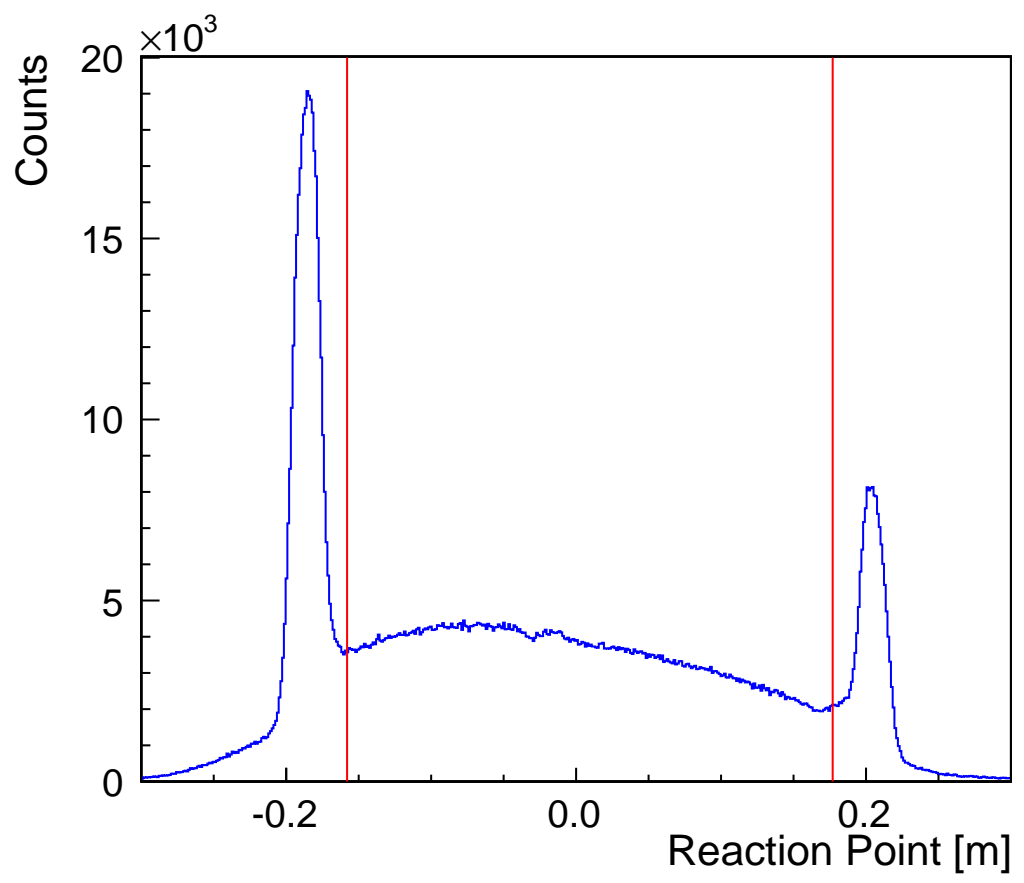

Figure 6.11: An example of the vertex cut.

Table 6.4: Summary of parameters used for pion rejecter/shower cut.

\begin{tabular}{|c|cc|cc|}
\hline & \multicolumn{2}{|c|}{ LHRS } & \multicolumn{2}{c|}{ RHRS } \\
\hline $\mathrm{E}(\mathrm{GeV})$ & $\mathrm{k}_{i}$ & $\mathrm{~b}_{i}$ & $\mathrm{k}_{i}$ & $\mathrm{~b}_{i}$ \\
\hline 1.245 & 1.1 & 600 & 0.8 & 600 \\
2.425 & 1.3 & 800 & 0.8 & 800 \\
3.605 & 1.3 & 1600 & 1.2 & 1600 \\
\hline
\end{tabular}

RHRS: R.cer.asum_c $>350$.

Lead-Glass Shower Detector Cut: This is a combination cut on the digitized calibrated ADC signals of pion rejecter layer 1 and pion rejecter layer 2 .

LHRS: L.prl1.e+k $\mathrm{k}_{i} \cdot$ L.prl2.e $>\mathrm{b}_{i}$

RHRS: R.ps.e $+\mathrm{k}_{i} \cdot$ R.sh.e $>\mathrm{b}_{i}$,

where $\mathrm{k}_{i}$ and $\mathrm{b}_{i}$ are summarized in Table 6.4. 


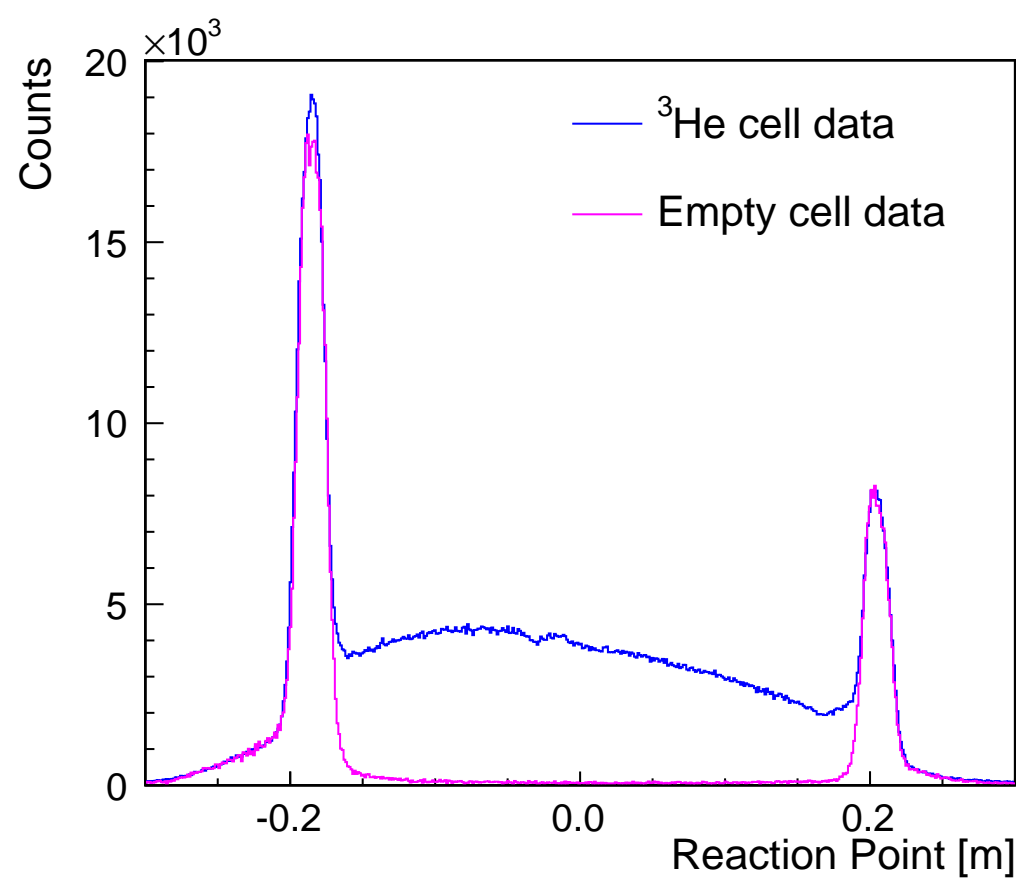

(a)

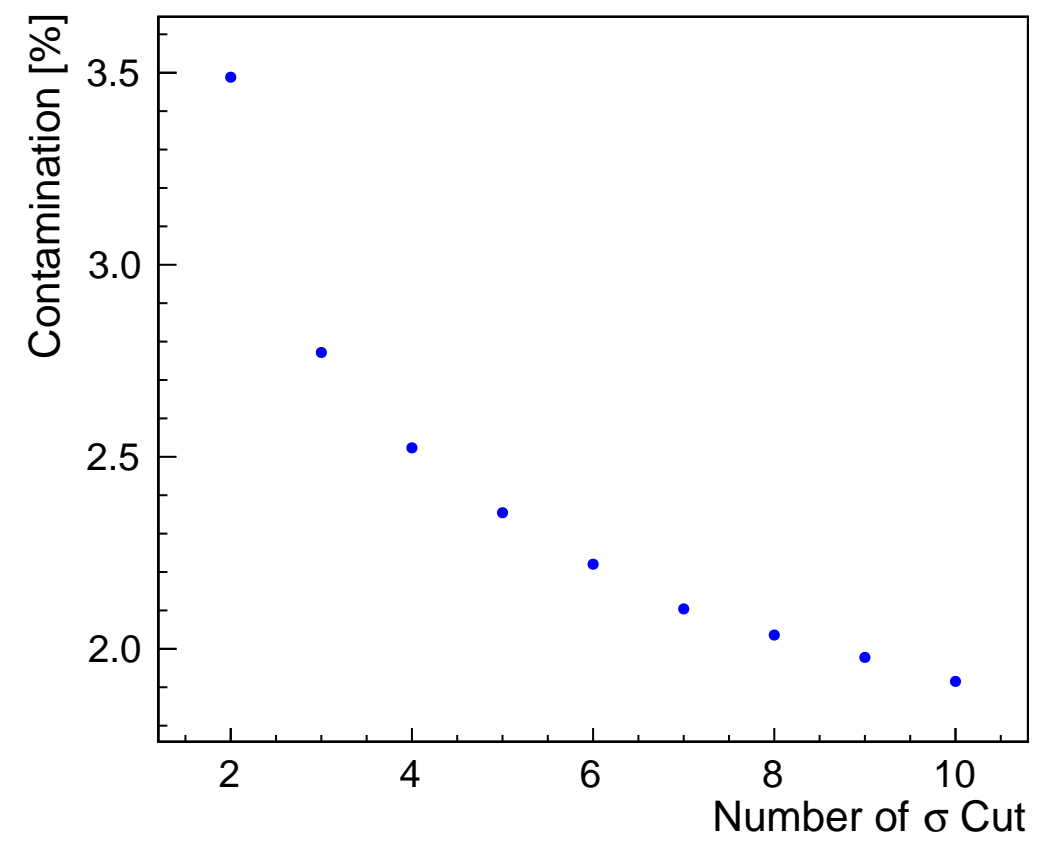

(b)

Figure 6.12: (a) Comparison of the data from ${ }^{3} \mathrm{He}$ target cell and empty target cell. (b) The glass contamination vs. $\sigma$ cut. 
Table 6.5: Summary of the binning of the energy transfer $\omega$ (in MeV) in E05-015.

\begin{tabular}{|c|cccc|}
\hline $\mathrm{E}(\mathrm{GeV})$ & bin\#1 & bin \#2 & bin \#3 & bin \#4 \\
\hline 1.245 & Elastic & $(35.0,65.0)$ & $(65.0,95.0)$ & $(95.0,125.0)$ \\
2.425 & $(120.0,180.0)$ & $(180.0,240.0)$ & $(240.0,300.0)$ & $(300.0,360.0)$ \\
3.605 & $(370.0,450.0)$ & $(450.0,530.0)$ & $(530.0,610.0)$ & $(610.0,690.0)$ \\
\hline
\end{tabular}

\subsection{DAQ Live-Time}

To monitor the electronics dead time, an electronic dead time pulse (EDTP) signal was plugged into each trigger type to simulate a real trigger, and this signal was also recorded in the data stream. The live-time is calculated with

$$
L=\frac{N_{\mathrm{EDTP}}^{\text {accepted }}}{N_{\mathrm{EDTP}}^{\text {scaler }}},
$$

where $N_{\mathrm{EDTP}}^{\text {accepted }}$ is the number of events recorded in physics data stream for EDTP signal and $N_{\text {EDTP }}^{\text {scaler }}$ is the number of counts recorded in scalers for this trigger (EDTP signal).

\subsection{Data Binning and Kinematics}

In this analysis, four data bins were used. Binning was done using the reconstructed energy transfer of the particle $(\omega)$. Within each $\omega$ bin, each event has a set of reconstructed kinematic variables. The final central $\omega$ values for each bin were determined by averaging over all events in the bin. A summary of the $\omega$ ranges for each kinematics point is given in Table 6.5.

\subsection{Nitrogen and Glass Dilution Factor}

As we mentioned in Section 5.2, a small amount of nitrogen was filled in the ${ }^{3} \mathrm{He}$ cell as a buffering gas. Electrons are not only scattered by the ${ }^{3}$ He nuclei but also by the glass windows of the target cell and the $\mathrm{N}_{2}$ nuclei. The dilution corrections 
from the $\mathrm{N}_{2}$ and glass backgrounds is therefore introduced in order to extract the physics quasi-elastic asymmetry:

$$
f=\frac{Y^{\mathrm{N}_{2}}+Y^{\mathrm{empty}}}{Y^{3 \mathrm{He}}+Y^{\mathrm{N}_{2}}+Y^{\mathrm{empty}}}
$$

In order to determine the dilution factor, a reference cell was used in the experiment.

$$
\begin{aligned}
f & =\frac{\left(Y_{\text {ref }}^{\mathrm{N}_{2}}-Y_{\text {ref }}^{\text {empty }}\right) \cdot \frac{n_{\text {prod }}^{\mathrm{N}_{2}}}{n_{\text {ref }}^{\mathrm{N}_{2}}}+Y_{\text {ref }}^{\text {empty }}}{Y_{\text {prod }}^{3}+Y_{\text {prod }}^{\mathrm{N}_{2}}+Y_{\text {prod }}^{\text {empty }}} \\
= & \frac{Y_{\text {ref }}^{\mathrm{N}_{2}} \cdot \frac{n_{\text {prod }}^{\mathrm{N}_{2}}}{n_{\text {ref }}^{\mathrm{N}_{2}}}+Y_{\text {ref }}^{\text {empty }} \cdot\left(1-\frac{n_{\text {prod }}^{\mathrm{N}_{2}}}{n_{\text {ref }}^{\mathrm{N}_{2}}}\right)}{Y_{\text {prod }}^{3}+Y_{\text {prod }}^{\mathrm{N}_{2}}+Y_{\text {prod }}^{\text {empty }}},
\end{aligned}
$$

where $n_{\text {ref(prod) }}^{\mathrm{N}_{2}}$ is the $\mathrm{N}_{2}$ density in the reference (production) cell. During the experiment, $\mathrm{N}_{2}$ and empty reference cell runs were taken periodically to determine $Y_{\text {ref }}^{\mathrm{N}_{2}} / n_{\text {ref }}^{\mathrm{N}_{2}}$ and $Y_{\text {ref }}^{\mathrm{empty}}$.

The pressure of the reference cell was measured in psig't. The density, in units of amagats, is calculated from the pressure taking into account the temperature with

$$
n=\left(\frac{p+p_{0}}{p_{0}}\right)\left(\frac{T_{0}}{T}\right),
$$

where $n$ is the density in amagat, $p$ is the pressure of the cell (in units of psig), $p_{0}$ is $1 \mathrm{~atm}$ (or $14.7 \mathrm{psia}$ ). $T$ is the temperature of the reference cell, and $T_{0}$ is 273.15 K. During experiment E05-015, the reference cell was held at a temperature 310-315 K. Table 6.6 shows the nitrogen and glass dilution factors for the three different kinematic settings calculated from this method. The main systematic uncertainty is contributed by the following:

- a relative $10 \%$ uncertainty in $\mathrm{N}_{2}$ filling density in the production cell;

- an uncertainty in the filling pressure of the reference cell of \pm 1 psig;

- an uncertainty in the reference temperature of $\pm 2 \mathrm{~K}$;

\footnotetext{
${ }^{1}$ pound-force per square inch gauge.
} 
Table 6.6: Summary of the nitrogen and glass dilution factors.

\begin{tabular}{|c|cc|}
\multicolumn{3}{|c}{$\mathrm{E}=1.245 \mathrm{GeV}$} \\
\hline$\omega(\mathrm{MeV})$ & f-LHRS & f-RHRS \\
\hline Elastic & $0.064 \pm 0.007$ & $0.041 \pm 0.004$ \\
$(35.0,65.0)$ & $0.105 \pm 0.011$ & $0.103 \pm 0.012$ \\
$(65.0,95.0)$ & $0.074 \pm 0.009$ & $0.070 \pm 0.008$ \\
$(95.0,125.0)$ & $0.080 \pm 0.010$ & $0.113 \pm 0.014$ \\
\hline$(35.0,125.0)$ & $0.085 \pm 0.010$ & $0.090 \pm 0.011$ \\
\hline
\end{tabular}

\begin{tabular}{|c|cc|}
\multicolumn{3}{c}{$\mathrm{E}=2.425 \mathrm{GeV}$} \\
\hline$\omega(\mathrm{MeV})$ & f-LHRS & f-RHRS \\
\hline$(120.0,180.0)$ & $0.133 \pm 0.011$ & $0.131 \pm 0.013$ \\
$(180.0,240.0)$ & $0.069 \pm 0.006$ & $0.067 \pm 0.006$ \\
$(240.0,300.0)$ & $0.060 \pm 0.005$ & $0.056 \pm 0.005$ \\
$(300.0,360.0)$ & $0.081 \pm 0.007$ & $0.074 \pm 0.007$ \\
\hline$(120.0,360.0)$ & $0.069 \pm 0.006$ & $0.065 \pm 0.006$ \\
\hline
\end{tabular}

\begin{tabular}{|c|cc|}
\multicolumn{4}{c}{$\mathrm{E}=3.605 \mathrm{GeV}$} \\
\hline$\omega(\mathrm{MeV})$ & f-LHRS & f-RHRS \\
\hline$(360.0,440.0)$ & $0.085 \pm 0.008$ & $0.081 \pm 0.008$ \\
$(440.0,520.0)$ & $0.063 \pm 0.006$ & $0.060 \pm 0.006$ \\
$(520.0,600.0)$ & $0.067 \pm 0.006$ & $0.063 \pm 0.006$ \\
$(600.0,680.0)$ & $0.084 \pm 0.008$ & $0.079 \pm 0.008$ \\
\hline$(360.0,680.0)$ & $0.070 \pm 0.007$ & $0.066 \pm 0.007$ \\
\hline
\end{tabular}




\subsection{Monte Carlo Simulation}

In order to extract the physics asymmetry, one needs to correct the experimental asymmetry for radiative corrections (RC). In our case, the factor $R^{\text {ert }}$ introduced in Section 6.1 .3 needs to be calculated from the $\mathrm{RC}$ procedure. In this analysis, Monte Carlo simulation programs were built to:

- simulate the elastic radiative tail under the quasi-elastic spectrum and obtain the elastic tail dilution factor $R^{\text {ert }}$.

- find the radiative corrected kinematics for the quasi-elastic asymmetry.

\subsubsection{Radiative Corrections}

Radiative corrections are divided into two types: external and internal. The external corrections are due to the electron interacting with materials in its path. Internal corrections result from inherent complications in electron scattering such as the vacuum polarization (Figure $2.3(\mathrm{~d})$ ) and vertex correction (Figure 2.3 (e) and (f)).

External Radiative Correction: Before scattering from the polarized ${ }^{3} \mathrm{He}$ target, the electron loses energy by passing through different materials in its path. At the reaction vertex, the electron energy is less than the energy the electron had when it entered the Hall. After scattering, the electron also passes through materials, which results in additional energy loss before it is detected. This means that the scattered electron energy at the vertex is higher than what is actually detected. The external radiative corrections are applied to determine the true kinematics of the reaction. The majority of the external radiative correction is from energy loss from bremsstrahlung (electron straggling), but a small amount is also from energy loss due to ionization. Detail discussion about the energy loss can be found in [79] and 


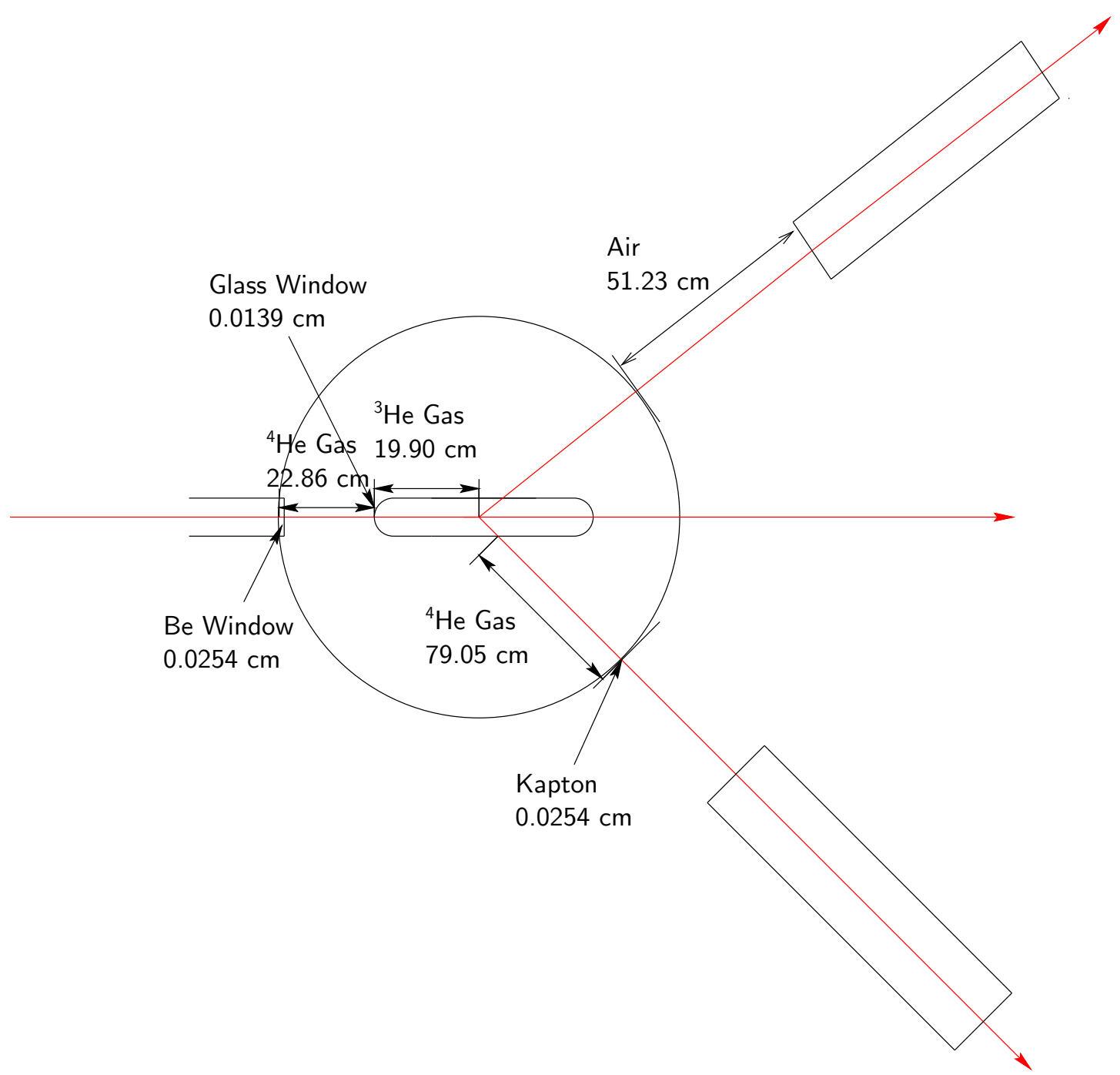

Figure 6.13: Schematic drawing of materials in particle's path before and after scattering.

[80]. A schematic drawing of the materials in electron's path is shown in Figure 6.13. The properties of materials the electrons pass through are shown in Table 6.7. The external radiative corrections were applied to each event in the Monte Carlo leading to a corrected distribution in the experiment.

Internal Radiative Correction Considering a typical $\left(e, e^{\prime}\right)$ scattering, most theoretical work calculates only the Born approximation, and not the higher order diagrams in Figure 2.3. However, in the experiment the higher order Feynman diagrams also contribute to this process. Thus, before carrying 
Table 6.7: Properties of radiation materials before and after scattering. $\rho$ is the density, $X_{0}$ is the radiation length, $L$ is the thickness of the material. The total thickness is in units of radiation lengths.

\begin{tabular}{|c|ccc|c|}
\hline material before scattering & $\begin{array}{c}\rho \\
\left(\mathrm{g} / \mathrm{cm}^{3}\right)\end{array}$ & $\begin{array}{c}\mathrm{L} \\
\left(\mathrm{g} / \mathrm{cm}^{2}\right)\end{array}$ & $\begin{array}{c}\mathrm{X}_{0} \\
(\mathrm{~cm})\end{array}$ & $\rho \cdot L / X_{0}$ \\
\hline $\mathrm{Be}$ & $1.848 \mathrm{E}+00$ & $2.540 \mathrm{E}-02$ & $6.519 \mathrm{E}+01$ & $7.200 \mathrm{E}-04$ \\
${ }^{4} \mathrm{He}$ & $1.664 \mathrm{E}-04$ & $2.286 \mathrm{E}+01$ & $9.432 \mathrm{E}+01$ & $4.033 \mathrm{E}-05$ \\
Glass & $2.760 \mathrm{E}+00$ & $1.390 \mathrm{E}-02$ & $2.684 \mathrm{E}+01$ & $1.429 \mathrm{E}-03$ \\
${ }^{3} \mathrm{He}$ & $1.654 \mathrm{E}-03$ & $1.990 \mathrm{E}+01$ & $7.107 \mathrm{E}+01$ & $4.631 \mathrm{E}-04$ \\
\hline Total & & & & $2.652 \mathrm{E}-03$ \\
\hline
\end{tabular}

\begin{tabular}{|c|ccc|c|}
\hline material after scattering & $\begin{array}{c}\rho \\
\left(\mathrm{g} / \mathrm{cm}^{3}\right)\end{array}$ & $\begin{array}{c}\mathrm{L} \\
\left(\mathrm{g} / \mathrm{cm}^{2}\right)\end{array}$ & $\begin{array}{c}\mathrm{X}_{0} \\
(\mathrm{~cm})\end{array}$ & $\rho \cdot L / X_{0}$ \\
\hline${ }^{3} \mathrm{He}$ & $1.654 \mathrm{E}-03$ & $3.207 \mathrm{E}+00$ & $7.107 \mathrm{E}+01$ & $7.464 \mathrm{E}-05$ \\
Glass & $2.760 \mathrm{E}+00$ & $5.746 \mathrm{E}-01$ & $2.684 \mathrm{E}+01$ & $5.909 \mathrm{E}-02$ \\
${ }^{4} \mathrm{He}$ & $1.664 \mathrm{E}-04$ & $7.905 \mathrm{E}+01$ & $9.432 \mathrm{E}+01$ & $1.395 \mathrm{E}-04$ \\
Kapton & $1.420 \mathrm{E}+00$ & $2.540 \mathrm{E}-02$ & $4.058 \mathrm{E}+01$ & $8.889 \mathrm{E}-04$ \\
Air & $1.210 \mathrm{E}-03$ & $5.123 \mathrm{E}+01$ & $3.666 \mathrm{E}+01$ & $1.691 \mathrm{E}-03$ \\
\hline Total & & & & $6.188 \mathrm{E}-02$ \\
\hline
\end{tabular}

out a comparison between the theoretical result and the experimental result, experimentalists perform "radiative corrections" on their experimental data. In our case we considered the corrections from the diagrams shown in Figure 6.14. Diagrams (a) and (b) are internal bremsstrahlung; they involve emission of real photons. Diagrams (c) and (d) show that the incident or scattered electron emit a virtual photon, and then the virtual photon is re-absorbed by the incident or scattered electron, which results in the re-normalization of the electron mass. Diagram (e) shows the incident electron emit a virtual photon and then the virtual photon is re-absorbed by the scattered electron, and gives rise to the vertex re-normalization. Diagram (f) shows the exchanged virtual photon creates a virtual positron-electron pair; it is called vacuum polarization. The diagrams where the virtual or emitted photons attach to the proton legs are smaller in magnitude due to the heavier proton mass, and are neglected. 


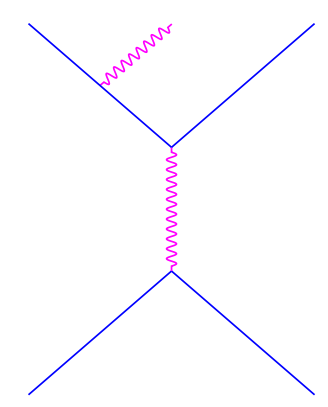

(a)

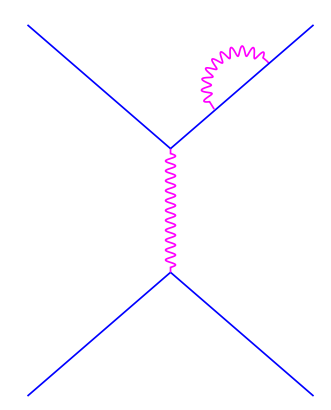

(d)

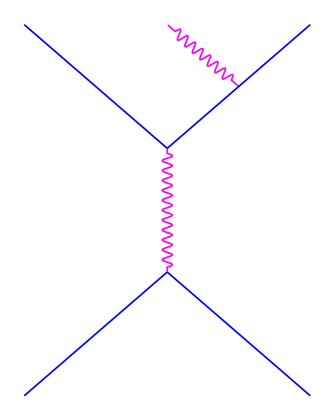

(b)

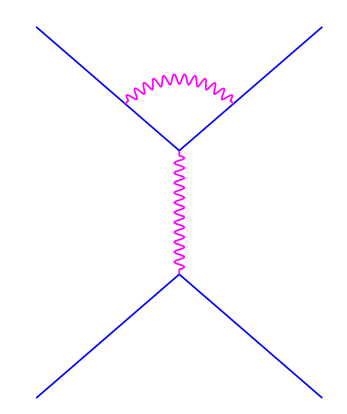

(e)

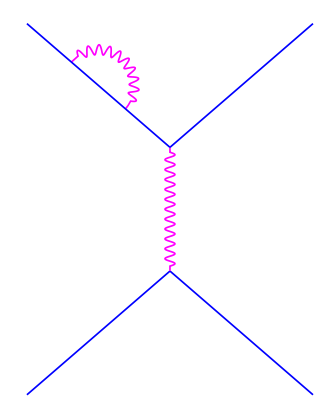

(c)

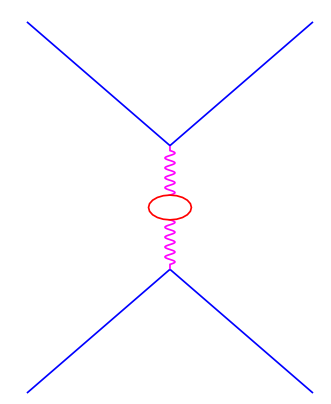

(f)

Figure 6.14: Second order Feynman diagrams considered for internal radiation correction. 


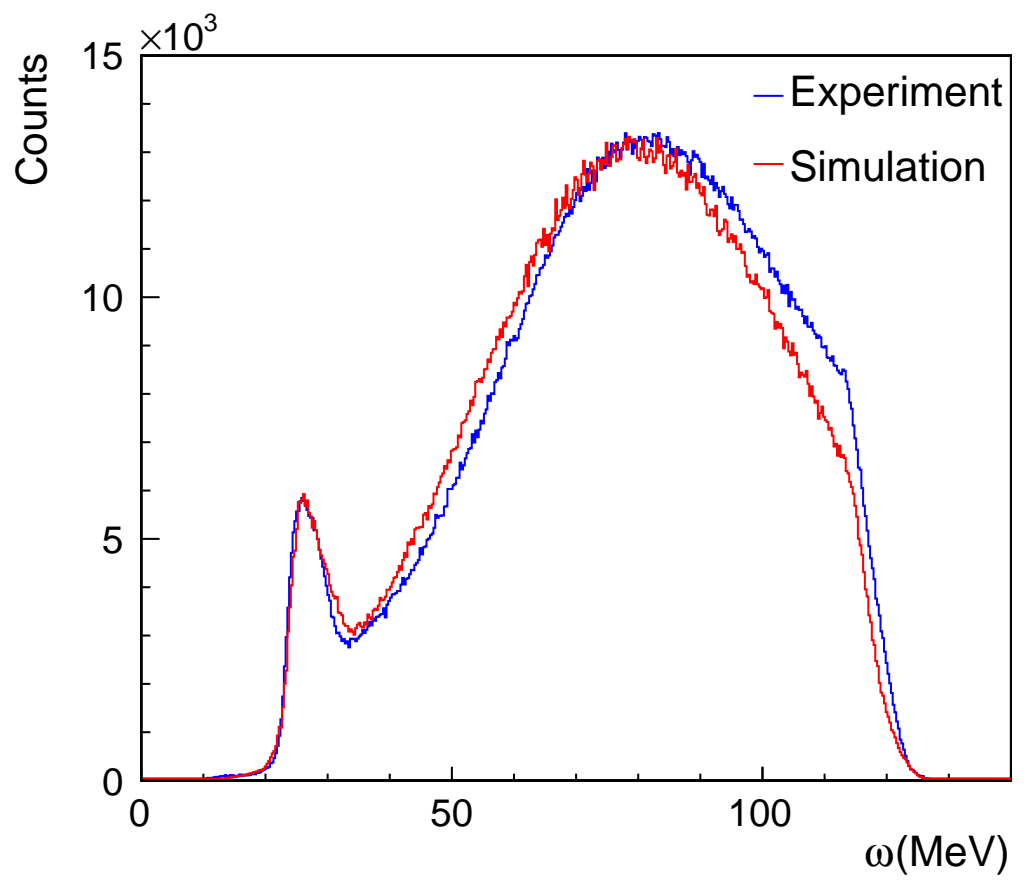

Figure 6.15: Comparison between data and simulation for both the elastic and quasi-elastic spectrum at $\mathrm{E}=1.245 \mathrm{GeV}$.

\subsubsection{Elastic Radiative Tail Dilution Factor}

With a precise knowledge of the ${ }^{3} \mathrm{He}$ elastic form factors and quasi-elastic spectral functions, we can calculate the elastic radiative tail dilution factor to a high precision, which is necessary for extracting the physics quasi-elastic asymmetry from the raw asymmetry as shown in Equation (6.16). During the simulation, we first calculate the ${ }^{3} \mathrm{He}$ Born cross-section using the ${ }^{3} \mathrm{He}$ elastic form factors, then we "radiate" this cross-section in order to compare with the data spectrum. The comparison can be seen in Figure 6.15. The elastic radiative tail dilution factors for each bin are shown in Table 6.8. For $E=2.425 \mathrm{GeV}$ and $E=3.605 \mathrm{GeV}$ data, the elastic radiative tail dilutions are negligible. 
Table 6.8: Summary of the elastic radiative tail dilution factors for $E=1.245$ $\mathrm{GeV}$. For $E=2.425 \mathrm{GeV}$ and $E=3.605 \mathrm{GeV}$ data, the elastic radiative tail dilution factors are negligible.

$\mathrm{E}=1.245 \mathrm{GeV}$
\begin{tabular}{|c|c|}
\hline$\omega(\mathrm{MeV})$ & $R^{e t}$ \\
\hline Elastic & - \\
$(35.0,65.0)$ & 0.072 \\
$(65.0,95.0)$ & 0.018 \\
$(95.0,125.0)$ & 0.018 \\
\hline$(35.0,125.0)$ & 0.031 \\
\hline
\end{tabular}

\subsubsection{Quasi-Elastic Asymmetry Radiative Correction}

The radiative corrected kinematics for quasi-elastic asymmetry are summarized in Table 6.9, where $\omega_{\text {exp-central }}$ represents the central $\omega$ value of the experiment and $\omega_{\text {corr-central }}$ represents the central $\omega$ value of the Born cross-section.

\subsection{Proton Dilution Factor}

In the extraction of the neutron single spin asymmetry from the measured ${ }^{3} \mathrm{He}$ asymmetry in the QE region, the common approach is to use the effective nucleon polarization:

$$
\begin{gathered}
\sigma^{3} \mathrm{He}=\sigma^{\mathrm{n}}+2 \sigma^{\mathrm{p}} \\
\Delta \sigma^{3} \mathrm{He}=P_{n} \cdot \Delta \sigma^{\mathrm{n}}+P_{p} \cdot 2 \sigma^{\mathrm{p}},
\end{gathered}
$$

where $\sigma^{3} \mathrm{He}, \sigma^{\mathrm{n}}$, and $\sigma^{\mathrm{p}}$ is the ${ }^{3} \mathrm{He}$ cross-section, neutron cross-section and proton cross-section, respectively. The effective neutron and proton polarizations in ${ }^{3} \mathrm{He}$ are given by $P_{n}=0.86_{-0.02}^{+0.036}$ and $P_{p}=-0.028_{-0.004}^{+0.009}[81$. Following this approach, the asymmetry of ${ }^{3} \mathrm{He}$ can be expressed using the asymmetry of the proton $\left(A^{\mathrm{p}}\right)$ and 
Table 6.9: Radiative correction to quasi-elastic asymmetry.

\begin{tabular}{|c|cc|}
\multicolumn{3}{|c}{$\mathrm{E}=1.245 \mathrm{GeV}$} \\
\hline$\omega(\mathrm{MeV})$ & $\omega_{\text {exp-central }}$ & $\omega_{\text {corr-central }}$ \\
\hline Elastic & - & - \\
$(35.0,65.0)$ & 54.6 & 54.2 \\
$(65.0,95.0)$ & 80.4 & 79.7 \\
$(95.0,125.0)$ & 105.4 & 104.3 \\
\hline$(35.0,125.0)$ & 81.8 & 78.4 \\
\hline
\end{tabular}

\begin{tabular}{|c|cc|}
\multicolumn{3}{c}{$\mathrm{E}=2.425 \mathrm{GeV}$} \\
\hline$\omega(\mathrm{MeV})$ & $\omega_{\text {exp-central }}$ & $\omega_{\text {corr-central }}$ \\
\hline$(120.0,180.0)$ & 168.1 & 167.8 \\
$(180.0,240.0)$ & 219.4 & 217.9 \\
$(240.0,300.0)$ & 269.9 & 267.7 \\
$(300.0,360.0)$ & 316.5 & 315.5 \\
\hline$(120.0,360.0)$ & 265.8 & 255.0 \\
\hline
\end{tabular}

\begin{tabular}{|c|cc|}
\multicolumn{3}{c}{$\mathrm{E}=3.605 \mathrm{GeV}$} \\
\hline$\omega(\mathrm{MeV})$ & $\omega_{\exp -\text { central }}$ & $\omega_{\text {corr-central }}$ \\
\hline$(360.0,440.0)$ & 419.1 & 418.0 \\
$(440.0,520.0)$ & 487.5 & 485.0 \\
$(520.0,600.0)$ & 560.4 & 558.5 \\
$(600.0,680.0)$ & 623.7 & 623.3 \\
\hline$(360.0,680.0)$ & 549.7 & 534.7 \\
\hline
\end{tabular}


Table 6.10: Rosenbluth cross-sections for nucleons.

\begin{tabular}{|c|c|c|c|ccc|}
\hline $\mathrm{Q}^{2}$ & $\mathrm{E}(\mathrm{GeV})$ & $\theta_{e^{\prime}}\left({ }^{\circ}\right)$ & $\mathrm{E}^{\prime}(\mathrm{GeV})$ & $\left.\sigma\right|_{p}\left(\frac{\mathrm{fm}^{2}}{\mathrm{sr}}\right)$ & $\left.\sigma\right|_{n}\left(\frac{\mathrm{fm}^{2}}{\mathrm{sr}}\right)$ & $\mathrm{f}_{n}$ \\
\hline 0.127 & 1.245 & 17 & 1.167 & $1.017 \times 10^{-2}$ & $1.077 \times 10^{-3}$ & 0.050 \\
0.460 & 2.425 & 17 & 2.170 & $1.001 \times 10^{-3}$ & $2.651 \times 10^{-4}$ & 0.117 \\
0.967 & 3.605 & 17 & 3.070 & $1.506 \times 10^{-4}$ & $5.695 \times 10^{-5}$ & 0.159 \\
\hline
\end{tabular}

the neutron $\left(A^{\mathrm{n}}\right)$ as

$$
A_{y}^{3 \mathrm{He}}=P_{n} \cdot f_{n} \cdot A_{y}^{\mathrm{n}}+P_{p} \cdot\left(1-f_{n}\right) \cdot A_{y}^{\mathrm{p}}
$$

where the neutron factor is defined as $f_{n}=\sigma^{\mathrm{n}} / \sigma^{3} \mathrm{He}$.

Following Equation (6.27), the neutron asymmetry was extracted from the ${ }^{3} \mathrm{He}$ asymmetry using the effective polarization approximation, given by

$$
A_{y}^{\mathrm{n}}=\frac{A_{y}^{3} \mathrm{He}}{f_{n} \cdot P_{n}}-\frac{\left(1-f_{n}\right) \cdot P_{p} \cdot A_{y}^{\mathrm{p}}}{f_{n} \cdot P_{n}},
$$

where the neutron factor $f_{n}$ was simply calculated using the Kelly parametrization [17] in identical kinematics with:

$$
f_{n}=\frac{\sigma^{\mathrm{n}}}{\sigma^{3} \mathrm{He}}=\frac{\sigma^{\mathrm{n}}}{2 \sigma^{\mathrm{p}}+\sigma^{\mathrm{n}}} .
$$

The parameters are shown in Table 6.10. For the $\mathrm{E}=1.245 \mathrm{GeV}$ data, since the $Q^{2}$ is very low, final state interactions are much more important. Hence, models are needed to calculate $\mathrm{f}_{n}$. A theoretical estimate [82] is $\mathrm{f}_{n}=0.042$.

$A_{y}^{p}\left(A_{y}^{n}\right)$ was predicted to be $0.01 \%(0.16 \%), 0.24 \%(-0.15 \%)$ at the first two kinematic settings $(\mathrm{E}=1.245 \mathrm{GeV}$ and $\mathrm{E}=2.425 \mathrm{GeV})$ by Andrei Afanasev [83], using the elastic intermediate state calculation. For the last kinematic setting $(\mathrm{E}=3.605 \mathrm{GeV}), A_{y}^{p}\left(A_{y}^{n}\right)$ was predicted by Afanasev to be $0.62 \%$ (-1.35\%) using the elastic intermediate state calculation plus a GPD model calculation. 


\subsection{Systematic Uncertainties}

This section summarizes the systematic uncertainties that are associated with the target single-spin asymmetry measurement.

Pion Contamination from PID Cuts: The purpose of the PID analysis was to reject as many pions as possible, while maintaining a high electron efficiency. Even with good PID cuts, a small amount of pions can make it through the cuts. The combination of Cherenkov and pion rejecter PID cuts typically reduces the pion to electron ratio by a factor of $10^{-4}$ [84]. Using this factor, the pion contamination to the electron asymmetry can be calculated by the following [85]:

$$
\Delta A=10^{-4} A^{\pi}
$$

The same cuts that were applied to the electron asymmetry determination were also used to evaluate the pion asymmetry, except for the Cherenkov cut and pion rejectors cut. In pion selection, the Cherenkov was cut at channel 0, which means the event had no signal in the Cherenkov detector. No cut was put on the pion rejector for pion selection. We found that the pion correction to the electron asymmetry is very small for all kinematics and hence has been neglected.

Systematic uncertainty from scalars (charge and live-time): As described at the beginning of this chapter, for each target spin state, the yield $(Y)$ is weighted by the charge $(C)$ and live-time $(L)$, which are calculated from the scalar information. Since there are two copies for each type of scaler and they have been checked for consistency, either of them can be used to calculate the charge and live-time. Moreover, for the charge, all $\mathrm{u} 1, \mathrm{u} 3$, $\mathrm{u} 10, \mathrm{~d} 1, \mathrm{~d} 3$, and $\mathrm{d} 10$ can be used in the calculation. In general, the left-arm copy was used for the left-arm data, while the right-arm copy was used for 
the right-arm data. However, these copies can be exchanged to recalculate the charge and live-time, which are then used to recalculate the asymmetry. The difference between the original asymmetry and the recalculated asymmetry is quoted as a systematic uncertainty from the scalers as shown in the tables from Table 6.11 to Table 6.16.

Target Orientation: During experiment E05-015, the target magnetic field direction was not exactly "normal" to the reaction plane but was offset by a small angle $(\theta, \phi)$. Compass calibration measurements provided a precise direction of the holding magnetic field. During the measurement, two different compasses were used: a vertical compass was used to determine the vertical angle $\phi$, while a horizontal compass was used to determine the azimuthal angle $\theta$ of the magnetic field. The compass data was analyzed by Miha Mihovilovic [67] from the University of Ljubljana. It was found that $\phi=1.1^{\circ} \pm 0.30^{\circ}$ for vertical + field direction and $179.4^{\circ} \pm 0.30^{\circ}$ for vertical - field direction. The systematic uncertainty from target misalignment is considered small, and not included in the error budget.

Target Polarization: Form Table 5.10, the systematic uncertainty from the target polarization is $5.6 \%$ relative. It is the main contributor to the overall systematic uncertainty.

Density Fluctuation Target density fluctuations can affect the particle detection rates, and hence introduce a false asymmetry. This asymmetry is $3.6 \times 10^{-5}$.

Glass and Nitrogen Dilution: The systematic uncertainties from the glass and nitrogen dilution are summarized in Table 6.6.

Difference from LHRS results and RHRS results: LHRS results and RHRS results were combined to get the final ${ }^{3} \mathrm{He}$ asymmetry. The difference of 
LHRS and RHRS results is quoted as a systematic uncertainty, i.e., $|\delta A|=$ $\frac{|| A_{\text {LHRS }}|-| A_{\text {RHRS }}||}{2}$.

${ }^{3} \mathbf{H e} \rightarrow \mathbf{n}:$ The difference of $\mathrm{f}_{n}$ obtained from the Kelly form factor parameterization and from Deltuva's model [82] were quoted as a systematic uncertainty for the $\mathrm{E}=1.245 \mathrm{GeV}$ data. Also, a $100 \%$ uncertainty was assumed for $A_{y}^{p}$.

Each of these uncertainties contribute to the overall uncertainty as:

$$
\begin{aligned}
\left(\delta A^{\text {sys }}\right)^{2}= & \left(\delta A_{C}\right)^{2}+\left(\delta A_{L T}\right)^{2}+\left(\delta A_{\text {ori. }}\right)^{2}+\left(\delta A_{\text {dens. }}\right)^{2} \\
& +\left(\frac{\partial A}{\partial f}\right)^{2}(\delta f)^{2}+\left(\frac{\partial A}{\partial P}\right)^{2}(\delta P)^{2} \\
& +\left(\frac{\partial A}{\partial f_{n}}\right)^{2}\left(\delta f_{n}\right)^{2}+\left(\frac{\partial A}{\partial A_{y}^{p}}\right)^{2}\left(\delta A_{y}^{p}\right)^{2}
\end{aligned}
$$

Table 6.11: LHRS $A_{y}^{3} \mathrm{He}$ systematic uncertainty budget at $\mathrm{E}=1.245 \mathrm{GeV}$.

\begin{tabular}{|c|c|cccc|c|}
\hline Source & Format & Elastic & $(35.0,65.0)$ & $(65.0,95.0)$ & $(95.0,125.0)$ & $(35.0,125.0)$ \\
\hline Charge & $\left|\delta A_{\text {exp }} / A_{\text {exp }}\right|$ & $3.0 \%$ & $1.8 \%$ & $1.8 \%$ & $1.0 \%$ & $1.5 \%$ \\
Livetime & $\left|\delta A_{\text {exp }} / A_{\text {exp }}\right|$ & $3.2 \%$ & $1.9 \%$ & $2.0 \%$ & $1.1 \%$ & $1.6 \%$ \\
Density Fluctuation & $\left|\delta A_{\text {exp }}\right|$ & $3.6 \times 10^{-5}$ & $3.6 \times 10^{-5}$ & $3.6 \times 10^{-5}$ & $3.6 \times 10^{-5}$ & $3.6 \times 10^{-5}$ \\
Nitrogen Dilution & $\left|\delta A_{\text {exp }} / A_{\text {exp }}\right|$ & $0.7 \%$ & $1.2 \%$ & $1.0 \%$ & $1.1 \%$ & $1.1 \%$ \\
Target Polarization & $\left|\delta A_{\text {exp }} / A_{\text {exp }}\right|$ & $5.6 \%$ & $5.6 \%$ & $5.6 \%$ & $5.6 \%$ & $5.6 \%$ \\
\hline Total & $\left|\delta A_{\text {exp }} / A_{\text {exp }}\right|$ & $10.4 \%$ & $7.7 \%$ & $7.8 \%$ & $6.4 \%$ & $7.1 \%$ \\
\hline
\end{tabular}

Table 6.12: RHRS $A_{y}^{3} \mathrm{He}$ systematic uncertainty budget at $\mathrm{E}=1.245 \mathrm{GeV}$.

\begin{tabular}{|c|c|cccc|c|}
\hline Source & Format & Elastic & $(35.0,65.0)$ & $(65.0,95.0)$ & $(95.0,125.0)$ & $(35.0,125.0)$ \\
\hline Charge & $\left|\delta A_{\text {exp }} / A_{\text {exp }}\right|$ & $1.8 \%$ & $1.0 \%$ & $1.0 \%$ & $1.0 \%$ & $1.0 \%$ \\
Livetime & $\left|\delta A_{\text {exp }} / A_{\text {exp }}\right|$ & $1.8 \%$ & $1.0 \%$ & $1.0 \%$ & $1.0 \%$ & $1.0 \%$ \\
Density Fluctuation & $\left|\delta A_{\text {exp }}\right|$ & $3.6 \times 10^{-5}$ & $3.6 \times 10^{-5}$ & $3.6 \times 10^{-5}$ & $3.6 \times 10^{-5}$ & $3.6 \times 10^{-5}$ \\
Nitrogen Dilution & $\left|\delta A_{\text {exp }} / A_{\text {exp }}\right|$ & $0.4 \%$ & $1.3 \%$ & $0.9 \%$ & $1.6 \%$ & $1.2 \%$ \\
Target Polarization & $\left|\delta A_{\text {exp }} / A_{\text {exp }}\right|$ & $5.6 \%$ & $5.6 \%$ & $5.6 \%$ & $5.6 \%$ & $5.6 \%$ \\
\hline Total & $\left|\delta A_{\text {exp }} / A_{\text {exp }}\right|$ & $7.7 \%$ & $6.4 \%$ & $6.3 \%$ & $6.5 \%$ & $6.4 \%$ \\
\hline
\end{tabular}


Table 6.13: LHRS $A_{y}^{3} \mathrm{He}$ systematic uncertainty budget at $\mathrm{E}=2.425 \mathrm{GeV}$.

\begin{tabular}{|c|c|cccc|c|}
\hline Source & Format & $(120.0,180.0)$ & $(180.0,240.0)$ & $(240.0,300.0)$ & $(300.0,360.0)$ & $(120.0,360.0)$ \\
\hline Charge & $\left|\delta A_{\text {exp }} / A_{\text {exp }}\right|$ & $0.4 \%$ & $1.3 \%$ & $1.2 \%$ & $2.8 \%$ & $1.3 \%$ \\
Livetime & $\left|\delta A_{\text {exp }} / A_{\text {exp }}\right|$ & $0.9 \%$ & $3.0 \%$ & $2.7 \%$ & $6.5 \%$ & $3.0 \%$ \\
Density Fluctuation & $\left|\delta A_{\text {exp }}\right|$ & $3.6 \times 10^{-5}$ & $3.6 \times 10^{-5}$ & $3.6 \times 10^{-5}$ & $3.6 \times 10^{-5}$ & $3.6 \times 10^{-5}$ \\
Nitrogen Dilution & $\left|\delta A_{\text {exp }} / A_{\text {exp }}\right|$ & $1.3 \%$ & $0.6 \%$ & $0.5 \%$ & $0.8 \%$ & $0.6 \%$ \\
Target Polarization & $\left|\delta A_{\text {exp }} / A_{\text {exp }}\right|$ & $5.6 \%$ & $5.6 \%$ & $5.6 \%$ & $5.6 \%$ & $5.6 \%$ \\
\hline Total & $\left|\delta A_{\text {exp }} / A_{\text {exp }}\right|$ & $5.9 \%$ & $6.8 \%$ & $6.6 \%$ & $10.0 \%$ & $6.8 \%$ \\
\hline
\end{tabular}

Table 6.14: RHRS $A_{y}^{3} \mathrm{He}$ systematic uncertainty budget at $\mathrm{E}=2.425 \mathrm{GeV}$.

\begin{tabular}{|c|c|cccc|c|}
\hline Source & Format & $(120.0,180.0)$ & $(180.0,240.0)$ & $(240.0,300.0)$ & $(300.0,360.0)$ & $(120.0,360.0)$ \\
\hline Charge & $\left|\delta A_{\text {exp }} / A_{\text {exp }}\right|$ & $0.3 \%$ & $0.9 \%$ & $1.3 \%$ & $2.5 \%$ & $1.2 \%$ \\
Livetime & $\left|\delta A_{\text {exp }} / A_{\text {exp }}\right|$ & $0.3 \%$ & $0.9 \%$ & $1.3 \%$ & $2.5 \%$ & $1.2 \%$ \\
Density Fluctuation & $\left|\delta A_{\text {exp }}\right|$ & $3.6 \times 10^{-5}$ & $3.6 \times 10^{-5}$ & $3.6 \times 10^{-5}$ & $3.6 \times 10^{-5}$ & $3.6 \times 10^{-5}$ \\
Nitrogen Dilution & $\left|\delta A_{\text {exp }} / A_{\text {exp }}\right|$ & $1.5 \%$ & $0.6 \%$ & $0.5 \%$ & $0.8 \%$ & $0.6 \%$ \\
Target Polarization & $\left|\delta A_{\text {exp }} / A_{\text {exp }}\right|$ & $5.6 \%$ & $5.6 \%$ & $5.6 \%$ & $5.6 \%$ & $5.6 \%$ \\
\hline Total & $\left|\delta A_{\text {exp }} / A_{\text {exp }}\right|$ & $5.8 \%$ & $5.9 \%$ & $6.3 \%$ & $7.8 \%$ & $6.2 \%$ \\
\hline
\end{tabular}

Table 6.15: LHRS $A_{y}^{3} \mathrm{He}$ systematic uncertainty budget at $\mathrm{E}=3.605 \mathrm{GeV}$.

\begin{tabular}{|c|c|cccc|c|}
\hline Source & Format & $(360.0,440.0)$ & $(440.0,520.0)$ & $(520.0,600.0)$ & $(600.0,680.0)$ & $(360.0,680.0)$ \\
\hline Charge & $\left|\delta A_{\text {exp }} / A_{\text {exp }}\right|$ & $1.4 \%$ & $0.9 \%$ & $1.0 \%$ & $1.2 \%$ & $1.0 \%$ \\
Livetime & $\left|\delta A_{\text {exp }} / A_{\text {exp }}\right|$ & $4.2 \%$ & $2.7 \%$ & $2.9 \%$ & $3.7 \%$ & $3.0 \%$ \\
Density Fluctuation & $\left|\delta A_{\text {exp }}\right|$ & $3.6 \times 10^{-5}$ & $3.6 \times 10^{-5}$ & $3.6 \times 10^{-5}$ & $3.6 \times 10^{-5}$ & $3.6 \times 10^{-5}$ \\
Nitrogen Dilution & $\left|\delta A_{\text {exp }} / A_{\text {exp }}\right|$ & $0.9 \%$ & $0.6 \%$ & $0.6 \%$ & $0.9 \%$ & $0.8 \%$ \\
Target Polarization & $\left|\delta A_{\text {exp }} / A_{\text {exp }}\right|$ & $5.6 \%$ & $5.6 \%$ & $5.6 \%$ & $5.6 \%$ & $5.6 \%$ \\
\hline Total & $\left|\delta A_{\text {exp }} / A_{\text {exp }}\right|$ & $7.6 \%$ & $6.5 \%$ & $6.6 \%$ & $7.2 \%$ & $6.7 \%$ \\
\hline
\end{tabular}

Table 6.16: RHRS $A_{y}^{3} \mathrm{He}$ systematic uncertainty budget at $\mathrm{E}=3.605 \mathrm{GeV}$.

\begin{tabular}{|c|c|cccc|c|}
\hline Source & Format & $(360.0,440.0)$ & $(440.0,520.0)$ & $(520.0,600.0)$ & $(600.0,680.0)$ & $(360.0,680.0)$ \\
\hline Charge & $\left|\delta A_{\text {exp }} / A_{\text {exp }}\right|$ & $0.5 \%$ & $1.2 \%$ & $0.9 \%$ & $1.1 \%$ & $1.0 \%$ \\
Livetime & $\left|\delta A_{\text {exp }} / A_{\text {exp }}\right|$ & $0.5 \%$ & $1.2 \%$ & $0.9 \%$ & $1.1 \%$ & $1.0 \%$ \\
Density Fluctuation & $\left|\delta A_{\text {exp }}\right|$ & $3.6 \times 10^{-5}$ & $3.6 \times 10^{-5}$ & $3.6 \times 10^{-5}$ & $3.6 \times 10^{-5}$ & $3.6 \times 10^{-5}$ \\
Nitrogen Dilution & $\left|\delta A_{\text {Exp }} / A_{\text {Exp }}\right|$ & $0.9 \%$ & $0.6 \%$ & $0.6 \%$ & $0.9 \%$ & $0.7 \%$ \\
Target Polarization & $\left|\delta A_{\text {Exp }} / A_{\text {Exp }}\right|$ & $5.6 \%$ & $5.6 \%$ & $5.6 \%$ & $5.6 \%$ & $5.6 \%$ \\
\hline Total & $\left|\delta A_{\text {Exp }} / A_{\text {Exp }}\right|$ & $5.8 \%$ & $6.3 \%$ & $6.0 \%$ & $6.2 \%$ & $6.1 \%$ \\
\hline
\end{tabular}


Table 6.17: Combined $A_{y}^{3} \mathrm{He}$ systematic uncertainty budget.

\begin{tabular}{|c|c|c|c|c|}
\hline Source & Format & $\mathrm{E}=1.245 \mathrm{GeV}$ & $\mathrm{E}=2.425 \mathrm{GeV}$ & $\mathrm{E}=3.605 \mathrm{GeV}$ \\
\hline Table 6.11 \& Table 6.12 & \multirow{3}{*}{$\left|\delta A_{E x p}\right|$} & \multirow{3}{*}{$0.8 \times 10^{-4}$} & \multirow{3}{*}{$1.2 \times 10^{-4}$} & \multirow{3}{*}{$1.3 \times 10^{-4}$} \\
\hline Table 6.13 \& Table 6.14 & & & & \\
\hline Table 6.15\&Table 6.16 & & & & \\
\hline LHRS-RHRS Difference & $\left|\delta A_{E x p}\right|$ & $2.4 \times 10^{-4}$ & $0.4 \times 10^{-4}$ & $0.2 \times 10^{-4}$ \\
\hline Total & $\left|\delta A_{\text {Exp }}\right|$ & $2.6 \times 10^{-4}$ & $1.3 \times 10^{-4}$ & $1.3 \times 10^{-4}$ \\
\hline
\end{tabular}

Table 6.18: Neutron asymmetry systematic uncertainty budget.

\begin{tabular}{|c|c|ccc|}
\hline Source & Format & $\mathrm{E}=1.245 \mathrm{GeV}$ & $\mathrm{E}=2.425 \mathrm{GeV}$ & $\mathrm{E}=3.605 \mathrm{GeV}$ \\
\hline$A_{y}^{3} \mathrm{He}$ & $\left|\delta A_{\text {Exp }}\right|$ & $2.6 \times 10^{-4}$ & $1.3 \times 10^{-4}$ & $1.3 \times 10^{-4}$ \\
$\mathrm{f}_{n}$ & $\left|\delta A_{\text {exp }} / A_{\text {exp }}\right|$ & $16.0 \%$ & - & - \\
$A_{y}^{p}$ & $\left|\delta A_{\text {exp }} / A_{\text {exp }}\right|$ & $100 \%$ & $100 \%$ & $100 \%$ \\
\hline Total & $\left|\delta A_{\text {exp }}\right|$ & $8.4 \times 10^{-3}$ & $1.3 \times 10^{-3}$ & $0.9 \times 10^{-3}$ \\
\hline
\end{tabular}




\section{Chapter 7}

\section{Results and Conclusions}

The results from experiment E05-015 will be presented in this chapter. Following the asymmetry extraction steps as described in Section 6.1, we first present the raw ${ }^{3}$ He asymmetry results, $A_{y}^{\text {raw }}$, from LHRS and RHRS in Section 7.1. Then the ${ }^{3} \mathrm{He}$ asymmetry, $A_{y}^{3 \mathrm{He}}$, will be presented in Section 7.2 . This asymmetry is corrected by the target polarization and nitrogen dilution factors. The ${ }^{3} \mathrm{He}$ asymmetries from LHRS and RHRS are combined in Section 7.3. After subtracting the radiative elastic tail, the ${ }^{3} \mathrm{He}$ asymmetry in quasi-elastic region, $A_{y}^{q e}$, will be presented in Section 7.4. Since the elastic tail contribution in $\mathrm{E}=2.425 \mathrm{GeV}$ and $\mathrm{E}=3.605 \mathrm{GeV}$ is very small and we neglect it in our analysis. The $A_{y}^{q e}$ results for $\mathrm{E}=2.425 \mathrm{GeV}$ and $\mathrm{E}=3.605 \mathrm{GeV}$ are the same as the $A_{y}^{3 \mathrm{He}}$ results as presented in Section 7.3. Using the effective polarization method, the neutron asymmetries are extracted and presented in Section 7.5. Finally, we conclude this dissertation in Section 7.6. 


\subsection{Raw Asymmetry $\left(A_{y}^{r a w}\right)$}

${ }^{3}$ He raw asymmetries, $A_{y}^{\text {raw }}$, defined by Equation (6.1), are given in Table 7.1 and Table 7.2 for the LHRS and the RHRS, respectively.

Table 7.1: LHRS $A_{y}^{\text {raw }}$ results summary.

\begin{tabular}{|c|c|cc|}
\multicolumn{5}{c}{$\mathrm{E}=1.245 \mathrm{GeV}$} \\
\hline$\omega(\mathrm{MeV})$ & $\langle\omega(\mathrm{MeV})\rangle$ & $A_{y}^{\text {raw }}(\%)$ & Stat. $(\%)$ \\
\hline Elastic & 25.8 & 0.023 & 0.041 \\
$(35.0,65.0)$ & 54.2 & -0.038 & 0.026 \\
$(65.0,95.0)$ & 79.7 & -0.037 & 0.019 \\
$(95.0,125.0)$ & 104.3 & -0.066 & 0.026 \\
\hline$(35.0,125.0)$ & 78.4 & -0.045 & 0.010 \\
\hline
\end{tabular}

$$
\mathrm{E}=2.425 \mathrm{GeV}
$$

\begin{tabular}{|c|c|cc|}
\hline$\omega(\mathrm{MeV})$ & $\langle\omega(\mathrm{MeV})\rangle$ & $A_{y}^{\text {raw }}(\%)$ & Stat. $(\%)$ \\
\hline$(120.0,180.0)$ & 167.8 & -0.259 & 0.082 \\
$(180.0,240.0)$ & 217.9 & -0.086 & 0.026 \\
$(240.0,300.0)$ & 267.7 & -0.096 & 0.019 \\
$(300.0,360.0)$ & 315.5 & -0.038 & 0.030 \\
\hline$(120.0,360.0)$ & 255.0 & -0.086 & 0.014 \\
\hline
\end{tabular}

\begin{tabular}{|c|c|cc|}
\multicolumn{5}{c}{$\mathrm{E}=3.605 \mathrm{GeV}$} \\
\hline$\omega(\mathrm{MeV})$ & $\langle\omega(\mathrm{MeV})>$ & $A_{y}^{\text {raw }}(\%)$ & Stat. $(\%)$ \\
\hline$(360.0,440.0)$ & 418.0 & -0.066 & 0.060 \\
$(440.0,520.0)$ & 485.0 & -0.107 & 0.024 \\
$(520.0,600.0)$ & 558.5 & -0.097 & 0.020 \\
$(600.0,680.0)$ & 623.3 & -0.076 & 0.028 \\
\hline$(360.0,680.0)$ & 534.7 & -0.094 & 0.013 \\
\hline
\end{tabular}


Table 7.2: RHRS $A_{y}^{\text {raw }}$ results summary.

\begin{tabular}{|c|c|cc|}
\multicolumn{5}{c}{$\mathrm{E}=1.245 \mathrm{GeV}$} \\
\hline$\omega(\mathrm{MeV})$ & $\langle\omega(\mathrm{MeV})\rangle$ & $A_{y}^{\text {raw }}(\%)$ & Stat. $(\%)$ \\
\hline Elastic & 25.8 & -0.039 & 0.040 \\
$(35.0,65.0)$ & 54.2 & 0.067 & 0.028 \\
$(65.0,95.0)$ & 79.7 & 0.071 & 0.020 \\
$(95.0,125.0)$ & 104.3 & 0.065 & 0.026 \\
\hline$(35.0,125.0)$ & 78.4 & 0.068 & 0.010 \\
\hline
\end{tabular}

$\mathrm{E}=2.425 \mathrm{GeV}$

\begin{tabular}{|c|c|cc|}
\hline$\omega(\mathrm{MeV})$ & $\langle\omega(\mathrm{MeV})\rangle$ & $A_{y}^{\text {raw }}(\%)$ & Stat. $(\%)$ \\
\hline$(120.0,180.0)$ & 167.8 & 0.333 & 0.093 \\
$(180.0,240.0)$ & 217.9 & 0.124 & 0.027 \\
$(240.0,300.0)$ & 267.7 & 0.083 & 0.019 \\
$(300.0,360.0)$ & 315.5 & 0.042 & 0.029 \\
\hline$(120.0,360.0)$ & 255.0 & 0.090 & 0.014 \\
\hline
\end{tabular}

\begin{tabular}{|c|c|cc|}
\multicolumn{5}{c}{$\mathrm{E}=3.605 \mathrm{GeV}$} \\
\hline$\omega(\mathrm{MeV})$ & $<\omega(\mathrm{MeV})>$ & $A_{y}^{\text {raw }}(\%)$ & Stat. $(\%)$ \\
\hline$(360.0,440.0)$ & 418.0 & 0.183 & 0.063 \\
$(440.0,520.0)$ & 485.0 & 0.077 & 0.024 \\
$(520.0,600.0)$ & 558.5 & 0.109 & 0.020 \\
$(600.0,680.0)$ & 623.3 & 0.082 & 0.027 \\
\hline$(360.0,680.0)$ & 534.7 & 0.097 & 0.013 \\
\hline
\end{tabular}




\section{$7.2 \quad{ }^{3}$ He Experimental Asymmetry $\left(A_{y}^{3} \mathrm{He}\right)$}

Using Equation (6.9), we made nitrogen dilution and target polarization corrections for the ${ }^{3} \mathrm{He}$ raw results, $A_{y}^{\text {raw }}$, to obtain the ${ }^{3} \mathrm{He}$ asymmetries, $A_{y}^{3 \mathrm{He}}$, which are presented in Table 7.3 and Table 7.4 for the LHRS and the RHRS, respectively. The tabulated asymmetries are also shown in Figure 7.1, and also shown summed over the quasifree peak in Figure 7.2. It can be seen that the asymmetries measured in the two arms are consistent with each other with the expected sign change from the two spectrometers being on opposite sides of the beam line.

Table 7.3: LHRS $A_{y}^{3} \mathrm{He}$ results summary.

\begin{tabular}{|c|c|ccc|}
\hline \multicolumn{6}{|c|}{$\mathrm{E}=1.245 \mathrm{GeV}$} \\
\hline$\omega(\mathrm{MeV})$ & $\langle\omega(\mathrm{MeV})\rangle$ & $\mathrm{A}_{y}^{3} \mathrm{He}(\%)$ & Stat. $(\%)$ & Sys. $(\%)$ \\
\hline Elastic & 25.8 & 0.048 & 0.085 & 0.005 \\
$(35.0,65.0)$ & 54.2 & -0.081 & 0.058 & 0.006 \\
$(65.0,95.0)$ & 79.7 & -0.078 & 0.040 & 0.006 \\
$(95.0,125.0)$ & 104.3 & -0.140 & 0.056 & 0.009 \\
\hline$(35.0,125.0)$ & 78.4 & -0.096 & 0.022 & 0.007 \\
\hline
\end{tabular}

\begin{tabular}{|c|c|ccc|}
\multicolumn{6}{|c|}{$\mathrm{E}=2.425 \mathrm{GeV}$} \\
\hline$\omega(\mathrm{MeV})$ & $<\omega(\mathrm{MeV})>$ & $\mathrm{A}_{y}^{3} \mathrm{He}(\%)$ & Stat. $(\%)$ & Sys. $(\%)$ \\
\hline$(120.0,180.0)$ & 167.8 & -0.583 & 0.185 & 0.034 \\
$(180.0,240.0)$ & 217.9 & -0.180 & 0.054 & 0.012 \\
$(240.0,300.0)$ & 267.7 & -0.200 & 0.040 & 0.013 \\
$(300.0,360.0)$ & 315.5 & -0.083 & 0.064 & 0.008 \\
\hline$(120.0,360.0)$ & 255.0 & -0.181 & 0.028 & 0.012 \\
\hline
\end{tabular}

\begin{tabular}{|c|c|ccc|}
\multicolumn{5}{|c|}{$\mathrm{E}=3.605 \mathrm{GeV}$} \\
\hline$\omega(\mathrm{MeV})$ & $<\omega(\mathrm{MeV})>$ & $\mathrm{A}_{y}^{3} \mathrm{He}(\%)$ & Stat. $(\%)$ & Sys. $(\%)$ \\
\hline$(360.0,440.0)$ & 418.0 & -0.142 & 0.129 & 0.011 \\
$(440.0,520.0)$ & 485.0 & -0.221 & 0.050 & 0.014 \\
$(520.0,600.0)$ & 558.5 & -0.205 & 0.041 & 0.014 \\
$(600.0,680.0)$ & 623.3 & -0.160 & 0.059 & 0.012 \\
\hline$(360.0,680.0)$ & 534.7 & -0.197 & 0.027 & 0.013 \\
\hline
\end{tabular}


Table 7.4: RHRS $A_{y}^{3} \mathrm{He}$ results summary.

\begin{tabular}{|c|c|ccc|}
\hline \multicolumn{1}{|c|}{$\mathrm{E}=1.245 \mathrm{GeV}$} \\
\hline$\omega(\mathrm{MeV})$ & $\langle\omega(\mathrm{MeV})>$ & $\mathrm{A}_{y}^{{ }^{3} \mathrm{He}}(\%)$ & Stat. (\%) & Sys. $(\%)$ \\
\hline Elastic & 25.8 & 0.079 & 0.081 & 0.006 \\
$(35.0,65.0)$ & 54.2 & 0.146 & 0.062 & 0.009 \\
$(65.0,95.0)$ & 79.7 & 0.147 & 0.041 & 0.009 \\
$(95.0,125.0)$ & 104.3 & 0.143 & 0.057 & 0.009 \\
\hline$(35.0,125.0)$ & 78.4 & 0.146 & 0.021 & 0.009 \\
\hline
\end{tabular}

$$
\mathrm{E}=2.425 \mathrm{GeV}
$$

\begin{tabular}{|c|c|ccc|}
\hline$\omega(\mathrm{MeV})$ & $\langle\omega(\mathrm{MeV})\rangle$ & $\mathrm{A}_{y}^{3} \mathrm{He}(\%)$ & Stat. (\%) & Sys. (\%) \\
\hline$(120.0,180.0)$ & 167.8 & 0.750 & 0.209 & 0.044 \\
$(180.0,240.0)$ & 217.9 & 0.259 & 0.057 & 0.015 \\
$(240.0,300.0)$ & 267.7 & 0.172 & 0.039 & 0.011 \\
$(300.0,360.0)$ & 315.5 & 0.090 & 0.062 & 0.007 \\
\hline$(120.0,360.0)$ & 255.0 & 0.188 & 0.028 & 0.012 \\
\hline
\end{tabular}

\begin{tabular}{|c|c|ccc|}
\multicolumn{5}{c|}{$\mathrm{E}=3.605 \mathrm{GeV}$} \\
\hline$\omega(\mathrm{MeV})$ & $<\omega(\mathrm{MeV})>$ & $\mathrm{A}_{y}^{3} \mathrm{He}(\%)$ & Stat. (\%) & Sys. (\%) \\
\hline$(360.0,440.0)$ & 418.0 & 0.389 & 0.135 & 0.022 \\
$(440.0,520.0)$ & 485.0 & 0.161 & 0.050 & 0.010 \\
$(520.0,600.0)$ & 558.5 & 0.222 & 0.041 & 0.013 \\
$(600.0,680.0)$ & 623.3 & 0.177 & 0.058 & 0.011 \\
\hline$(360.0,680.0)$ & 534.7 & 0.201 & 0.027 & 0.012 \\
\hline
\end{tabular}



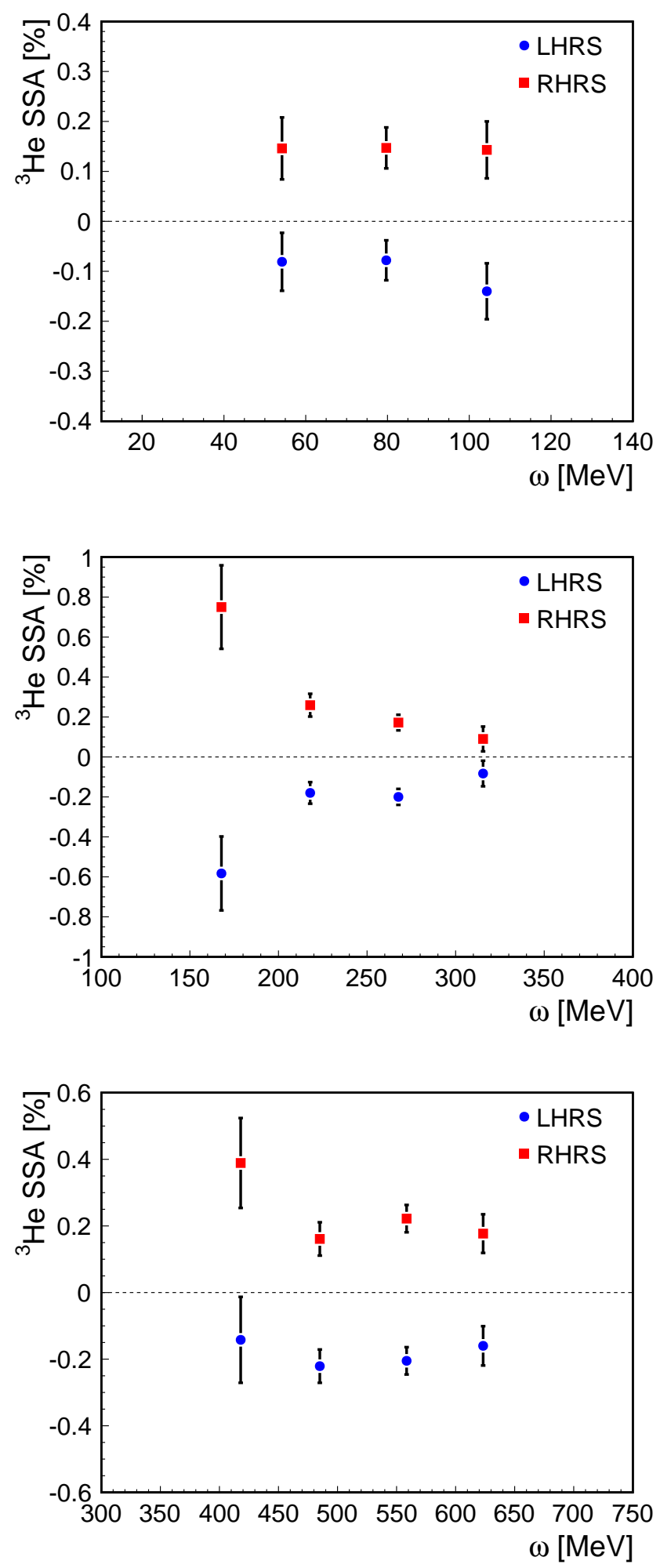

Figure 7.1: ${ }^{3} \mathrm{He}$ asymmetries from LHRS and RHRS plotted versus energy transfer $\omega$. The elastic radiative tail is not subtracted in this plot. The error bars represent the statistical uncertainties. 


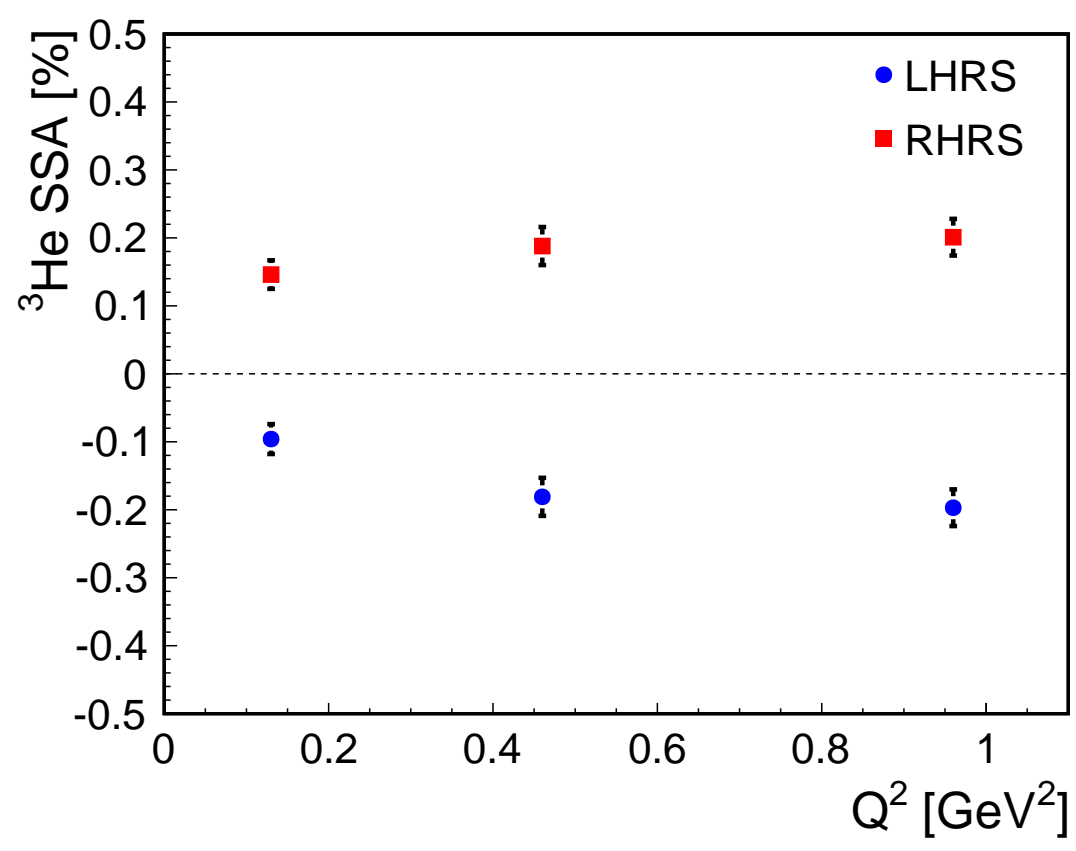

Figure 7.2: ${ }^{3} \mathrm{He}$ asymmetries from LHRS and RHRS as a function of 4-momentum transfer squared $\left(Q^{2}\right)$. The elastic radiative tail is not subtracted in this plot. The error bars represent the statistical uncertainties. 


\subsection{Combined LHRS and RHRS $A_{y}^{3} \mathrm{He}$}

The ${ }^{3} \mathrm{He}$ results presented in Section 7.2 are combined in this section, with the combined results presented in Table 7.5. $A_{y}^{3} \mathrm{He}$ from LHRS and RHRS are weighted by their statistics in order to get the combined asymmetries.

Table 7.5: $A_{y}^{3} \mathrm{He}$ results summary.

\begin{tabular}{|c|c|ccc|}
\multicolumn{6}{|c|}{$\mathrm{E}=1.245 \mathrm{GeV}$} \\
\hline$\omega(\mathrm{MeV})$ & $\langle\omega(\mathrm{MeV})>$ & $\mathrm{A}_{y}^{3} \mathrm{He}(\%)$ & Stat. $(\%)$ & Sys. $(\%)$ \\
\hline Elastic & 25.8 & 0.063 & 0.059 & 0.016 \\
$(35.0,65.0)$ & 54.2 & -0.115 & 0.042 & 0.033 \\
$(65.0,95.0)$ & 79.7 & -0.113 & 0.029 & 0.035 \\
$(95.0,125.0)$ & 104.3 & -0.142 & 0.040 & 0.009 \\
\hline$(35.0,125.0)$ & 78.4 & -0.120 & 0.015 & 0.026 \\
\hline
\end{tabular}

\begin{tabular}{|c|c|ccc|}
\multicolumn{6}{|c|}{$\mathrm{E}=2.425 \mathrm{GeV}$} \\
\hline$\omega(\mathrm{MeV})$ & $<\omega(\mathrm{MeV})>$ & $\mathrm{A}_{y}^{3} \mathrm{He}(\%)$ & Stat. $(\%)$ & Sys. $(\%)$ \\
\hline$(120.0,180.0)$ & 167.8 & -0.672 & 0.140 & 0.092 \\
$(180.0,240.0)$ & 217.9 & -0.221 & 0.039 & 0.042 \\
$(240.0,300.0)$ & 267.7 & -0.186 & 0.028 & 0.018 \\
$(300.0,360.0)$ & 315.5 & -0.086 & 0.045 & 0.008 \\
\hline$(120.0,360.0)$ & 255.0 & -0.185 & 0.020 & 0.013 \\
\hline
\end{tabular}

$\mathrm{E}=3.605 \mathrm{GeV}$

\begin{tabular}{|c|c|ccc|}
\hline$\omega(\mathrm{MeV})$ & $\langle\omega(\mathrm{MeV})\rangle$ & $\mathrm{A}_{y}^{3} \mathrm{He}(\%)$ & Stat. (\%) & Sys. (\%) \\
\hline$(360.0,440.0)$ & 418.0 & -0.268 & 0.093 & 0.125 \\
$(440.0,520.0)$ & 485.0 & -0.191 & 0.035 & 0.032 \\
$(520.0,600.0)$ & 558.5 & -0.214 & 0.029 & 0.016 \\
$(600.0,680.0)$ & 623.3 & -0.168 & 0.041 & 0.014 \\
\hline$(360.0,680.0)$ & 534.7 & -0.199 & 0.019 & 0.013 \\
\hline
\end{tabular}




\section{4 ${ }^{3} \mathrm{He}$ Experimental Asymmetry in the Quasi-Elastic Region $\left(A_{y}^{\mathrm{qe}}\right)$}

Equation (6.16) was used to correct the results presented in Section 7.3 for $\mathrm{E}=1.245 \mathrm{GeV}$ by the elastic tail dilution factor. The ${ }^{3} \mathrm{He}$ asymmetries in the quasi-elastic region after the correction are presented in Table 7.6. Recall that the elastic peak is small and the tail correction is insignificant for the higher beam energies. All beam energies are plotted in Figure 7.3 .

Table 7.6: Summary of Quasi-Elastic $A_{y}^{3} \mathrm{He}$ results for $\mathrm{E}=1.245 \mathrm{GeV}$ data.

\begin{tabular}{|c|c|ccc|}
\hline$\omega(\mathrm{MeV})$ & $<\omega(\mathrm{MeV})>$ & $\mathrm{A}_{y}^{3} \mathrm{He}(\%)$ & Stat. $(\%)$ & Sys. $(\%)$ \\
\hline Elastic & 25.8 & 0.063 & 0.059 & 0.016 \\
$(35.0,65.0)$ & 54.2 & -0.129 & 0.045 & 0.036 \\
$(65.0,95.0)$ & 79.7 & -0.116 & 0.030 & 0.036 \\
$(95.0,125.0)$ & 104.3 & -0.146 & 0.041 & 0.009 \\
\hline$(35.0,125.0)$ & 78.4 & -0.126 & 0.015 & 0.027 \\
\hline
\end{tabular}



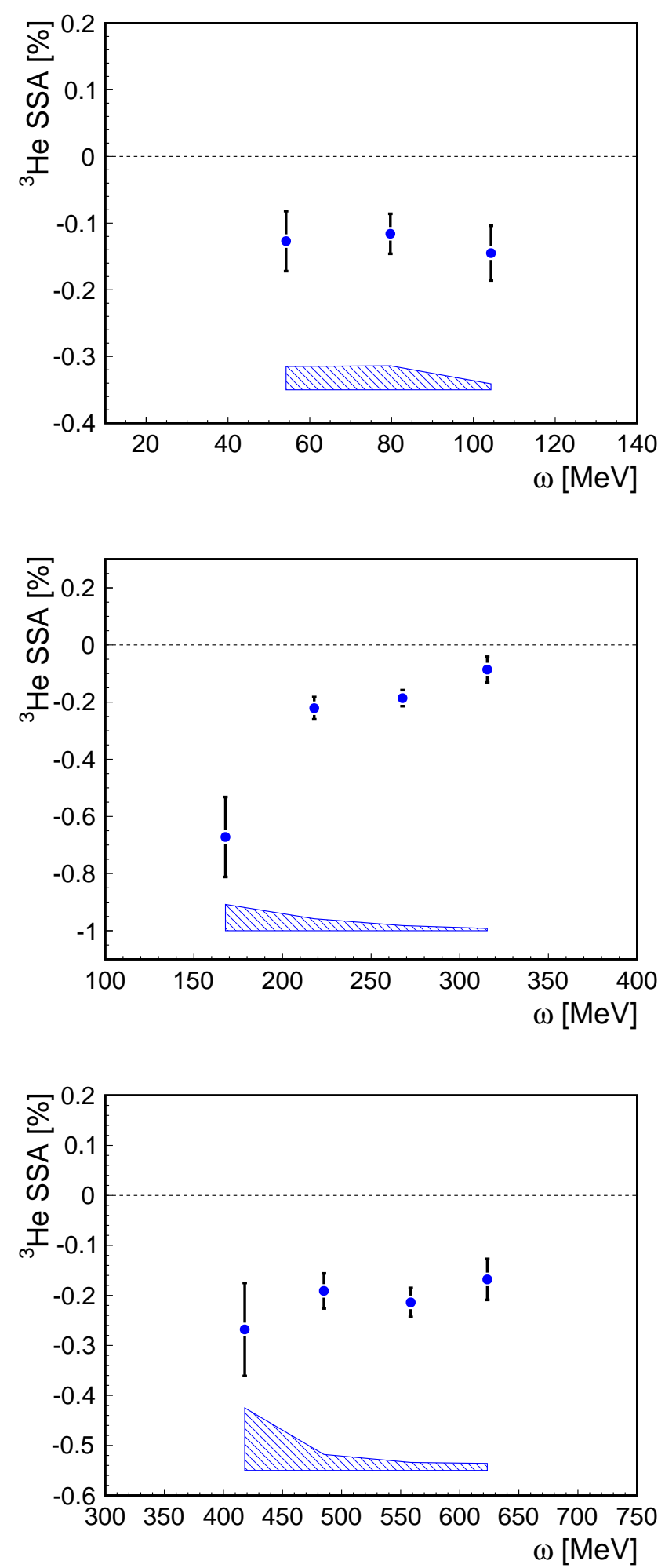

Figure 7.3: ${ }^{3} \mathrm{He}$ asymmetries in the quasi-elastic region are shown versus energy transfer $\omega$. The error bars indicate the statistical uncertainties only. The systematic uncertainties are indicated by the band below the data. 


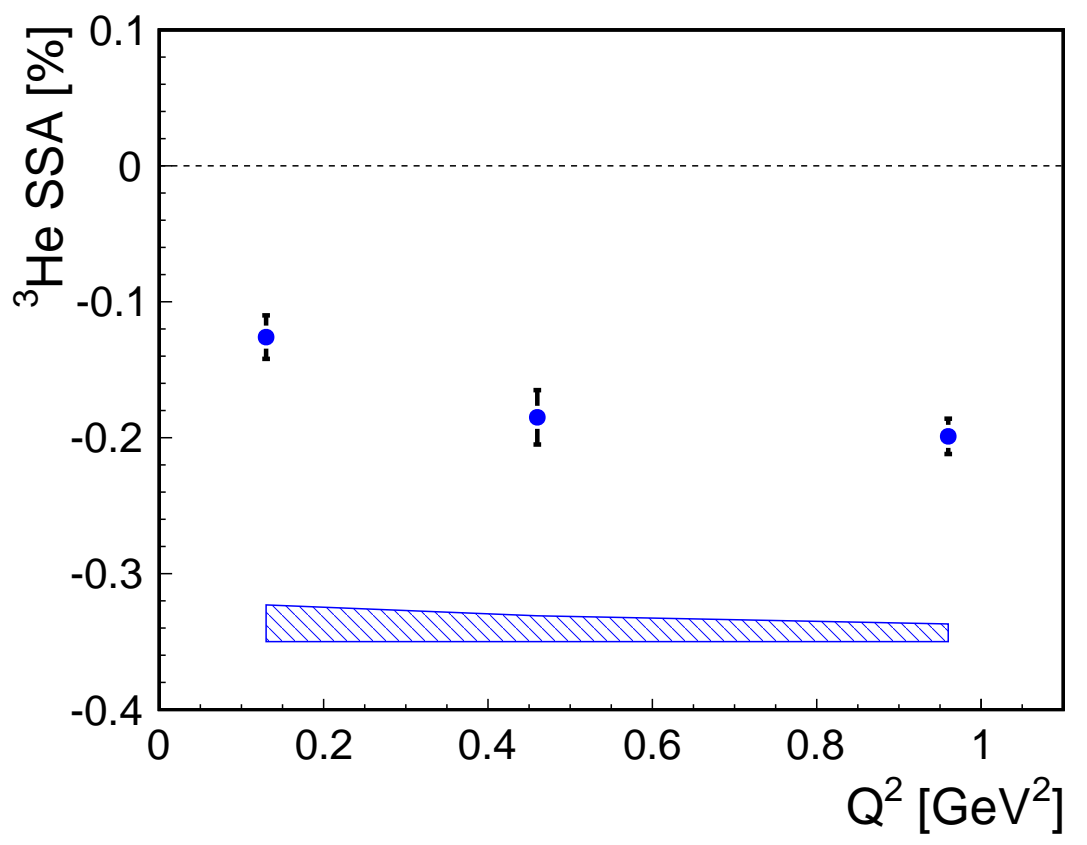

Figure 7.4: ${ }^{3} \mathrm{He}$ asymmetries summed over the quasi-elastic region plotted versus 4-momentum transfer squared $\left(Q^{2}\right)$. The error bars indicate the statistical uncertainties only. The systematic uncertainties are indicated by the band below the data. 


\subsection{Neutron Asymmetry in the Quasi-Elastic Region $\left(A_{y}^{n}\right)$}

The neutron asymmetry, $A_{y}^{n}$, was extracted using Equation $(6.28)$ from the quasielastic ${ }^{3}$ He results presented in Table $7.6(\mathrm{E}=1.245 \mathrm{GeV})$ and in Table $7.5(\mathrm{E}=2.425$ $\mathrm{GeV}$ and $\mathrm{E}=3.605 \mathrm{GeV}$ ). The $A_{y}^{n}$ results are presented in Table 7.7 and plotted in Figure 7.5 .

Table 7.7: Summary of results for the neutron asymmetry $A_{y}^{n}$.

\begin{tabular}{|c|ccc|}
\hline $\mathrm{E}(\mathrm{GeV})$ & $A_{y}^{n}(\%)$ & Stat. $(\%)$ & Sys. $(\%)$ \\
\hline 1.245 & -2.93 & 0.35 & 0.84 \\
2.425 & -1.84 & 0.20 & 0.13 \\
3.605 & -1.45 & 0.14 & 0.09 \\
\hline
\end{tabular}

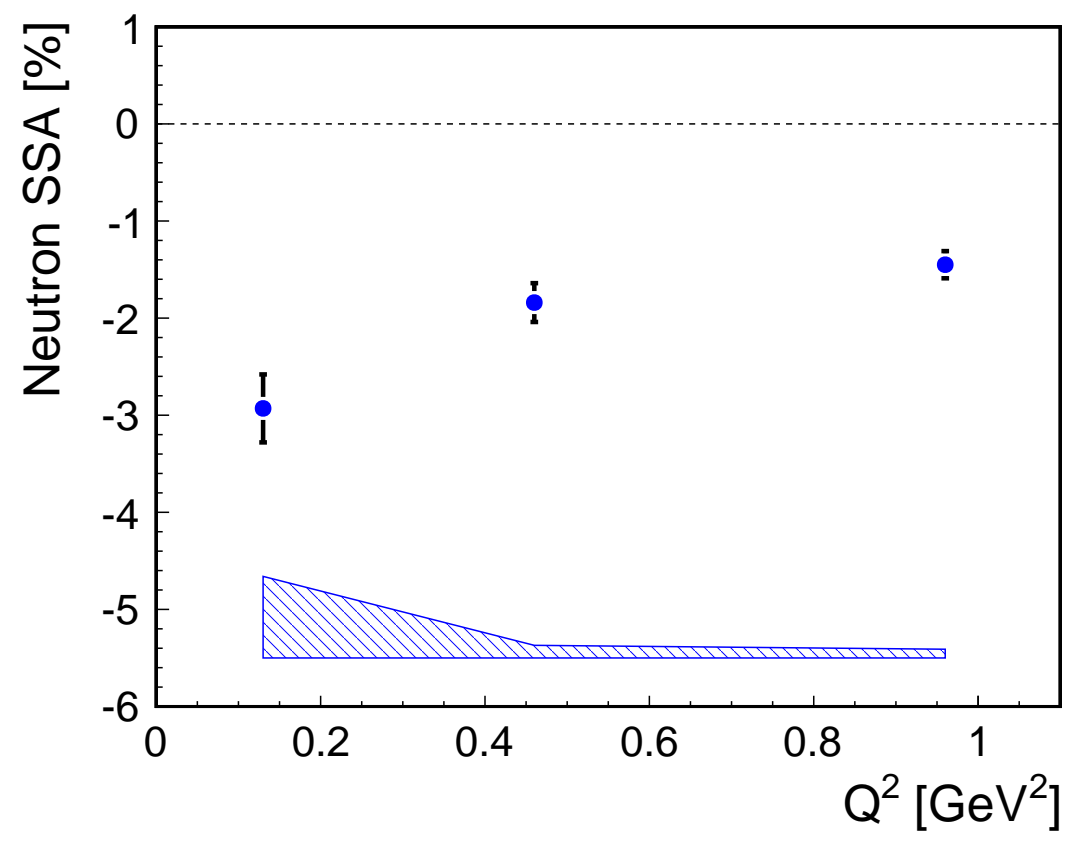

Figure 7.5: The neutron asymmetry $A_{y}^{n}$ as a function of 4-momentum transfer squared $\left(Q^{2}\right)$. The error bars indicate the statistical uncertainties only. The systematic uncertainties are indicated by the band below the data. 


\subsection{Conclusions and Outlook}

E05-015 has performed the first measurement of the target single-spin asymmetry, $A_{y}^{3} \mathrm{He}$, in the quasi-elastic region using a normally polarized ${ }^{3} \mathrm{He}$ target. We have presented a detailed description of the instrumentation and the detectors, as well as the ${ }^{3} \mathrm{He}$ target, that were used in the experiment. The procedures involved in calibrating the detectors and determining the target polarization have been discussed. The physics asymmetry analysis, which includes the selection of good events from the data using different cuts to determine the background and the false asymmetries, has been presented. A procedure to extract the neutron information from the measured ${ }^{3} \mathrm{He}$ asymmetries has been described. Finally, the asymmetries resulting from this experiment have been presented.

Negative asymmetries were observed at all $Q^{2}$ points for ${ }^{3} \mathrm{He}^{\uparrow}\left(e, e^{\prime}\right)$. Combining data from all $\omega-x$ bins, including both statistical and systematic uncertainties, we have observed for ${ }^{3} \mathrm{He}$ :

- a negative asymmetry with $4.1 \sigma$ significance at $Q^{2}=0.13 \mathrm{GeV}^{2}$.

- a negative asymmetry with $7.8 \sigma$ significance at $Q^{2}=0.46 \mathrm{GeV}^{2}$.

- a negative asymmetry with $8.6 \sigma$ significance at $Q^{2}=0.97 \mathrm{GeV}^{2}$.

and for the neutron:

- a negative asymmetry with $3.2 \sigma$ significance at $Q^{2}=0.13 \mathrm{GeV}^{2}$.

- a negative asymmetry with $7.7 \sigma$ significance at $Q^{2}=0.46 \mathrm{GeV}^{2}$.

- a negative asymmetry with $8.7 \sigma$ significance at $Q^{2}=0.97 \mathrm{GeV}^{2}$.

The final precision for $A_{y}^{n}$ is an order of magnitude improved over the existing proton data, as shown in Figure 7.6. The results are inconsistent with calculations by Afanasev [83] (Section 6.9) using only the elastic nucleon intermediate state, 
but agree for the last kinematic setting $\left(Q^{2}=0.97 \mathrm{GeV}^{2}\right)$ with calculations with an elastic nucleon intermediate state plus GPD model contributions.

$A_{y}$ provides a direct probe of the imaginary component of the $2 \gamma$ exchange amplitude. It is hoped that the results from E05-015 will stimulate theoretical efforts to understand $2 \gamma$ exchange, in particular the hadron structure dependent parts of the box and crossed-box diagrams neglected in the standard treatments of radiative corrections.

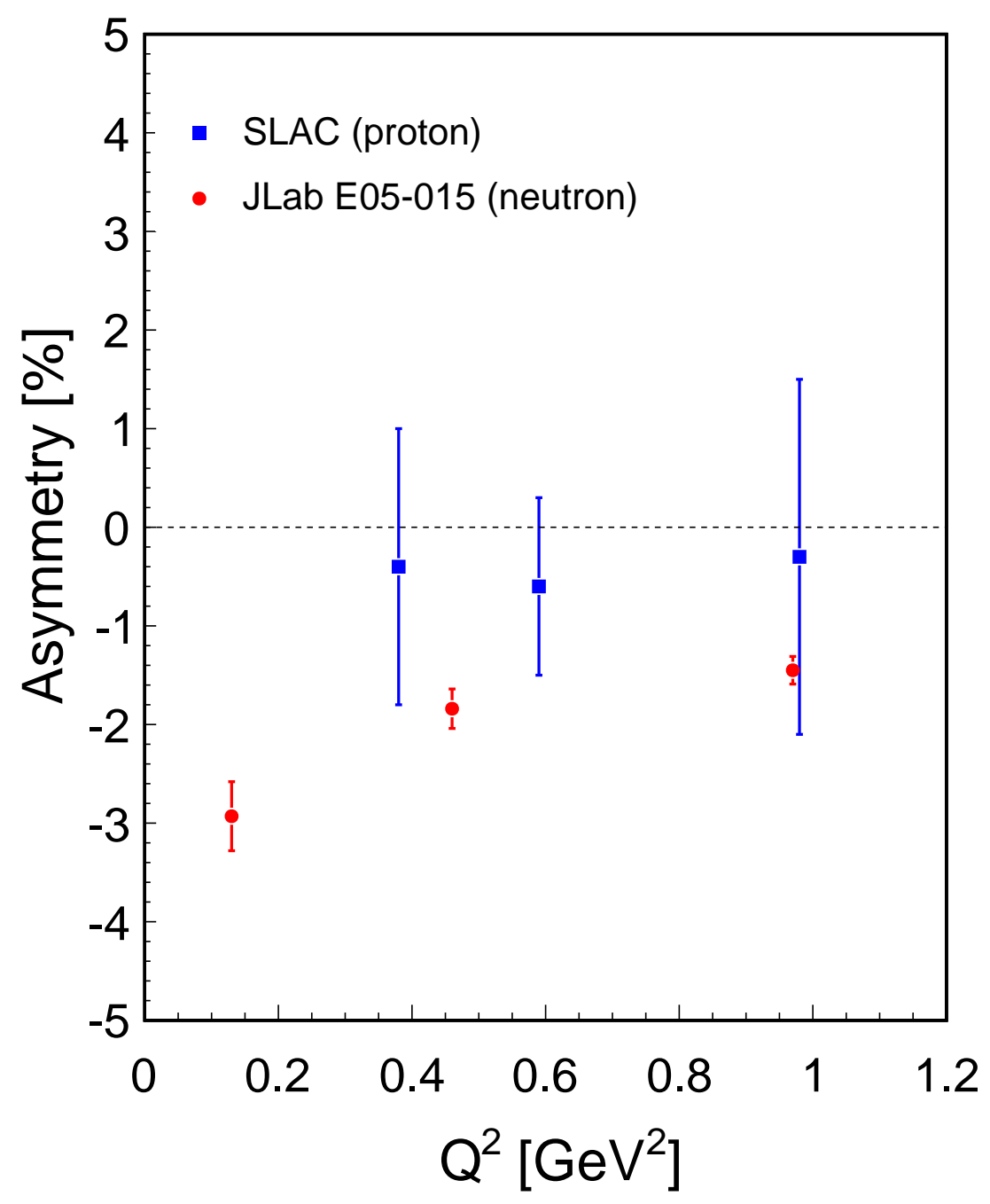

Figure 7.6: Neutron asymmetries of this work compared with the available proton asymmetry results. The error bars indicate the statistical uncertainties only. 


\section{References}

[1] T. Averett, J.-P. Chen, and X. Jiang, Measurement of the Target Single-Spin Asymmetry in Quasi-Elastic ${ }^{3} \mathrm{He}^{\uparrow}\left(e, e^{\prime}\right), 2005$.

[2] J. J. Thomson, Philosophical Magazine 44, 293 (1897).

[3] J. J. Thomson, Philosophical Magazine 7, 237 (1904).

[4] E. Rutherford, Philosophical Magazine 21, 669 (1911).

[5] J. Chadwick, Nature 129, 312 (1932).

[6] M. Gell-Mann, Phys. Lett. 8, 214 (1964).

[7] G. Zweig, CERN preprint TH, 401 (1964).

[8] P. Davies, The Forces of Nature (Cambridge Univ. Press, 2nd edition, 1986).

[9] J. Gao, Ph.D. thesis, Massachuetts Institute of Technology, MA, 1999.

[10] R. P. Feynman, Quantum Electrodynamics (Advanced Books Classics) (Westview Press, $1^{\text {st }}$ edition, 1998).

[11] B. Povh, K. Rith, C. Scholz, and F. Zetsche, Particles and Nuclei, An Introduction to the Physical Concepts (Springer, $5^{\text {th }}$ edition, 2006).

[12] R. P. Feynman, Phys. Rev. 76, 749 (1949).

[13] N. F. Mott and H. S. W. Massey, The Theory of Atomic Collisions (Oxford, $3^{\text {rd }}$ edition, 1965).

[14] J. D. Jackson, Classical Electrodynamics (Wiley, $3^{\text {rd }}$ edition, 1998).

[15] X. Zheng, Ph.D. thesis, Massachuetts Institute of Technology, MA, 2002.

[16] M. N. Rosenbluth, Phys. Rev. 79, 615 (1950).

[17] J. J. Kelly, Phys. Rev. C 70, 068202 (2004).

[18] R.-W. Schulze and P. U. Sauer, Phys. Rev. C 48, 38 (1993).

[19] C. Amsler et al., Phys. Lett. B 667, 1 (2008).

[20] M. Anselmino, A. Efremov, and E.Leader, Phys. Rept. A 261, 1 (1995). 
[21] F. Halzen and A. D. Martin, Quarks and Leptons: An Introductory Course in Modern Particle Physics (Wiley, $1^{\text {st }}$ edition, 1984).

[22] M. K. Jones et al., Phys. Rev. Lett. 84, 1398 (2000).

[23] O. Gayou et al., Phys. Rev. Lett. 88, 092301 (2002).

[24] P. G. Blunden, W. Melnitchouk, and J. A. Tjon, Phys. Rev. Lett. 91, 142304 (2003).

[25] P. A. M. Guichon and M. Vanderhaeghen, Phys. Rev. Lett. 91, 142303 (2003).

[26] J. Arrington, P. Blunden, and W. Melnitchouk, Prog. Part. Nucl. Phys. 66, 782 (2011).

[27] J. Arrington, Phys. Rev. C 68, 034325 (2003).

[28] R. C. Walker et al., Phys. Rev. D 49, 5671 (1994).

[29] Y.-C. Chen et al., Phys. Rev. Lett. 93, 122301 (2004).

[30] P. G. Blunden, W. Melnitchouk, and J. A. Tjon, Phys. Rev. C 72, 034612 (2005).

[31] J. Arrington, W. Melnitchouk, and J. A. Tjon, Phys. Rev. C 76, 035205 (2007).

[32] A. De Rujula, J. M. Kaplan, and E. De Merenkov, Nucl. Phys. B 53, 545 (1973).

[33] T. Powell et al., Phys. Rev. Lett. 24, 753 (1970).

[34] G. V. Di Giorgio et al., Nuovo Cimento 39, 474 (1965).

[35] J. C. Bizot et al., Phys. Rev. 140, B1387 (1965).

[36] D. E. Lundquist, R. L. Anderson, J. V. Allaby, and D. M. Ritson, Phys. Rev. 168, 1527 (1968).

[37] R. Prepost, R. M. Simonds, and B. H. Wiik, Phys. Rev. Lett. 21, 1271 (1968).

[38] S. P. Wells et al., Phys. Rev. C 63, 064001 (2001).

[39] F. E. Maas et al., Phys. Rev. Lett. 94, 082001 (2005).

[40] D. S. Armstrong et al., Phys. Rev. Lett. 99, 092301 (2007).

[41] M. C. Harvey, Ph.D. thesis, Hampton University, VA, 2001.

[42] J. M. Katich, Ph.D. thesis, The College of William and Mary, VA, 2011. 
[43] F. Gross, J. Phys. Conf. Ser. 299, 012001 (2011).

[44] C. K. Sinclair et al., Phys. Rev. ST Accel. Beams 10, 023501 (2007).

[45] R. Alley, H. Aoyagi, J. Clendenin, and J. Frisch, Nucl. Inst. \& Meth. in Phys. Res. A 365, 1 (1995).

[46] Y. Chao et al., J. Phys. Conf. Ser. 299, 012015 (2011).

[47] C. Hernandez-Garcia, M. L. Stutzman, and P. G. O'Shea, Phys. Today 61N2, 44 (2008).

[48] C. Leeman, D. Douglas, and G. Krafft, Annu. Rev. Nucl. Part. Sci. 51, 413 (2001).

[49] J. Alcorn et al., Nucl. Instrum. Meth. A522, 294 (2004).

[50] C. Dutta, Ph.D. thesis, University of Kentucky, 2010.

[51] K. Unser, IEEE Trans. Nucl. Sci. 28, 2344 (1981).

[52] J. Huang, Ph.D. thesis, Massachuetts Institute of Technology, MA, 2011.

[53] Hall A General Operations Mannual, 2011.

[54] K. Fissum et al., Nucl. Instrum. Meth. A474, 108 (2001).

[55] Y. Qiang, Ph.D. thesis, Massachuetts Institute of Technology, MA, 2007.

[56] M. Iodice et al., Nucl. Instrum. Meth. A411, 223 (1998).

[57] K. Nakamura and P. D. Group, Journal of Physics G: Nuclear and Particle Physics 37, 075021 (2010).

[58] B. Blankleider and R. M. Woloshyn, Phys. Rev. C 29, 538 (1984).

[59] J. L. Friar et al., Phys. Rev. C 42, 2310 (1990).

[60] M. A. Bouchiat, T. R. Carver, and C. M. Varnum, Phys. Rev. Lett. 5, 373 (1960).

[61] T. G. Walker, Phys. Rev. A 40, 4959 (1989).

[62] A. Ben-Amar Baranga et al., Phys. Rev. Lett. 80, 2801 (1998).

[63] W. C. Chen, T. R. Gentile, T. G. Walker, and E. Babcock, Phys. Rev. A 75, 013416 (2007).

[64] E. Babcock et al., Phys. Rev. Lett. 91, 123003 (2003).

[65] M. V. Romalis, Ph.D. thesis, Princeton University, NJ, 1997. 
[66] M. V. Romalis, E. Miron, and G. D. Cates, Phys. Rev. A 56, 4569 (1997).

[67] M. Mihovilovic, Ph.D. thesis, University of Ljubljana, Slovenia, 2012.

[68] J. Singh, Technical Report University of Virginia (2009).

[69] A. Abragam, Principles of Nuclear Magnetism (Oxford, $1^{\text {st }}$ edition, 1983).

[70] E. Babcock, I. A. Nelson, S. Kadlecek, and T. G. Walker, Phys. Rev. A 71, 013414 (2005).

[71] J. Singh, A. M. Kelleher, and P. H. Solvignon, Technical Report University of Virginia (2008).

[72] Q. Ye et al., Eur. Phys. J. A44, 55 (2010).

[73] N. R. Newbury et al., Phys. Rev. A 48, 4411 (1993).

[74] G. D. Cates et al., Phys. Rev. A 38, 5092 (1988).

[75] G. D. Cates, S. R. Schaefer, and W. Happer, Phys. Rev. A 37, 2877 (1988).

[76] Hall A Collaboration, ROOT/C++ Analyzer for Hall A http://hallaweb.jlab.org/podd/index.html .

[77] X. Qian, Ph.D. thesis, Duke University, NC, 2010.

[78] G. Jin, Ph.D. thesis, University of Virginia, VA, 2011.

[79] F. Xiong, Ph.D. thesis, Massachuetts Institute of Technology, MA, 2002.

[80] W. Xu, Ph.D. thesis, Massachuetts Institute of Technology, MA, 2002.

[81] X. Zheng et al., Phys. Rev. Lett. 92, 012004 (2004).

[82] A. Deltuva, Private Communication .

[83] A. Afanasev, Private Communication .

[84] Y.-W. Zhang, https://userweb.jlab.org/ yawei/weeklymeeting/111026/index.html

[85] P. H. Solvignon, Ph.D. thesis, Temple University, PA, 2006. 


\section{Appendix A Wall Thickness Measurement Results}

The following tables contain the wall thickness of the different measured cells. 
Table A.1: The reference cell GMB2 wall thickness.

\begin{tabular}{|c|cc|}
\hline Position & JLab Results $(\mathrm{mm})$ & UVA Results $(\mathrm{mm})$ \\
\hline $\mathrm{A}$ & 1.679 & 1.58 \\
$\mathrm{~B}$ & 1.699 & 1.66 \\
$\mathrm{C}$ & 1.702 & 1.67 \\
$\mathrm{D}$ & 1.712 & 1.51 \\
$\mathrm{E}$ & 1.591 & 1.61 \\
$\mathrm{~F}$ & 1.610 & 1.48 \\
$\mathrm{G}$ & 1.547 & 1.70 \\
$\mathrm{H}$ & 1.533 & 1.78 \\
$\mathrm{I}$ & 1.514 & 1.61 \\
$\mathrm{~J}$ & $\mathrm{~N} / \mathrm{A}$ & 1.63 \\
\hline Average & $1.621 \pm 0.075$ & $1.62 \pm 0.08$ \\
\hline $\mathrm{W} 1$ & 0.141 & 0.151 \\
$\mathrm{~W} 2$ & 0.134 & 0.132 \\
\hline
\end{tabular}

Table A.2: The ${ }^{3} \mathrm{He}$ cell Dominic wall thickness.

\begin{tabular}{|c|cc|}
\hline Position & JLab Results $(\mathrm{mm})$ & UVA Results $(\mathrm{mm})$ \\
\hline $\mathrm{A}$ & 1.691 & 1.55 \\
$\mathrm{~B}$ & 1.702 & 1.62 \\
$\mathrm{C}$ & 1.716 & 1.64 \\
$\mathrm{D}$ & 1.691 & 1.67 \\
$\mathrm{E}$ & 1.656 & 1.67 \\
$\mathrm{~F}$ & 1.598 & 1.62 \\
$\mathrm{G}$ & 1.710 & 1.72 \\
$\mathrm{H}$ & 1.695 & 1.63 \\
$\mathrm{I}$ & 1.662 & 1.63 \\
J & 1.654 & 1.75 \\
\hline Average & $1.638 \pm 0.035$ & $1.65 \pm 0.05$ \\
\hline W1 & 0.139 & 0.132 \\
W2 & 0.154 & 0.150 \\
\hline
\end{tabular}

